\title{
Late Cenozoic behaviour of two Transantarctic Mountain outlet glaciers
}

\author{
by \\ Richard Selwyn Jones
}
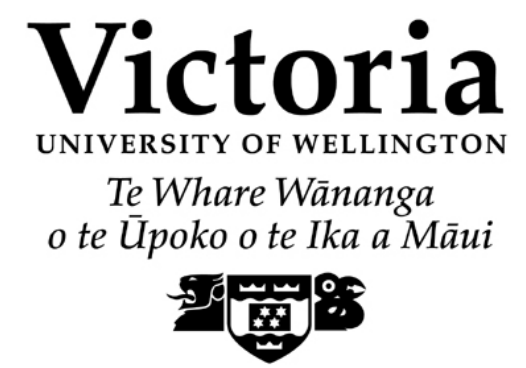

\author{
A thesis \\ submitted to Victoria University of Wellington \\ in fulfilment of the \\ requirements for the degree of \\ Doctor of Philosophy \\ in Physical Geography.
}

Victoria University of Wellington

2015 


\begin{abstract}
Earth's climate is undergoing dramatic warming that is unprecedented in at least the last 2000 years. Outlets of the Antarctic ice sheet are experiencing dynamic thinning, terminus retreat and mass loss, however, we are currently unable to accurately predict their future response. The drivers and mechanisms responsible for these observed changes can be better understood by studying the behaviour of outlet glaciers in the geological past. Here, I use cosmogenic nuclide surface-exposure dating and numerical glacier modelling to investigate the past configurations and dynamics of Transantarctic Mountain outlet glaciers, in the Ross Sea sector of Antarctica.
\end{abstract}

Numerical modelling was first applied to understand the present-day and past behaviour of Skelton Glacier. A suite of sensitivity experiments reveal that Skelton Glacier is most susceptible to atmospheric temperature through its affect on basal sliding near the groundingline. Under past climates, large changes occurred in the lower reaches of the glacier, with basal sliding and bedrock erosion predicted in the overdeepened basins during both the Pliocene and Quaternary. Skelton Glacier was likely much shorter and thinner during Pliocene interglacials, with warm-based sliding that extended along most of its length.

Informed by the glacier modelling, I applied surface-exposure dating to constrain past fluctuations in the geometry of Skelton Glacier. The lower reaches of the glacier were likely thicker at the Last Glacial Maximum (LGM), supporting the idea of buttressing by grounded ice in the Ross Sea during glacial periods. The glacier then thinned to near-modern surface elevations by $\sim 5.8$ ka before present (BP). Multiple isotope analysis $\left({ }^{26} \mathrm{Al}-{ }^{10} \mathrm{Be}\right)$ and exposure-burial modelling indicates that Skelton Glacier has fluctuated between interglacial and glacial configurations probably at orbital frequencies since the Miocene. These data record a total of $>10 \mathrm{Ma}$ of exposure and 2.5 Ma of burial. An unexpected outcome is that the average cosmogenic production rate over this time appears to have been at least twice that of today.

The long-term dynamics of Transantarctic Mountain outlet glaciers are further explored at Mackay Glacier. Here, geomorphological evidence reveals that glaciers can both erode and preserve bedrock surfaces during the same glacial episode, with basal erosion controlled primarily by ice thickness. Mackay Glacier likely experienced a widespread erosive regime prior to the Quaternary and a polythermal glacier regime during the LGM. 
Deglaciation following the LGM is constrained with $\left({ }^{10} \mathrm{Be}\right)$ surface-exposure dating at Mackay Glacier. Samples collected at two nunataks, across four transects, reveal glacier thinning of $>260 \mathrm{~m}$ between the LGM and $\sim 200$ years BP. Ice surface lowering was initially gradual, however an episode of rapid thinning is then recorded at $\sim 6.8 \mathrm{ka} \mathrm{BP}$, during a period of relative climatic and oceanic stability. This accelerated surface lowering occurred at a rate commensurate with modern observations of rapid ice sheet thinning, persisted for at least four centuries, and resulted in $>180 \mathrm{~m}$ of ice loss. Numerical modelling indicates that ice surface drawdown resulted from 'marine ice sheet instability' as the grounding-line retreated through a deep glacial trough on the inner continental-shelf.

This research provides new geological constraints and quantitative predictions of the past behaviour of Transantarctic Mountain outlet glaciers. The basal conditions and discharge of these glaciers evolved through the Late Cenozoic in response to climate forcing at orbital timescales, but also to topographically-controlled feedbacks at centennial to millennial timescales. Importantly, under enhanced atmospheric warming, these results imply that such outlet glaciers could experience greater ice loss through increased basal sliding and unstable grounding-line retreat into overdeepened basins. 


\section{Acknowledgements}

Research carried out for this thesis would not have been possible without support from the Victoria Doctoral Scholarship, Antarctic Endowed Development Fund, School of Geography, Environment and Earth Sciences, Antarctic Science Bursary, and Antarctica New Zealand.

Foremost, I thank my supervisors - Andrew Mackintosh, Kevin Norton and Nick Golledge - for guiding me through this work and sharing their passion for the subject. Andrew, your supervision and encouragement has been integral to the project and my professional development. Kevin, you trained me into a geochemist and also turned me into a coffee snob, and I am grateful for both. Nick, you patiently taught me the ways of glacier modelling and inspired me to make better salads.

I would like to acknowledge the staff and students in the Antarctic Research Centre for providing a friendly and motivational environment within which to work. Many laboratories and bottles of HF were used in the making of this thesis, and I therefore owe thanks to Ashley Pocock, Jane Chewings, Sabrina Lange and Kosta Tashkoff (SGEES, VUW), as well as Roger Tremain (GNS Science). I would like to thank Gary Wilson (Otago) and Chris Fogwill (UNSW) for assistance with fieldwork, and Euan Smith for that all-important statistical insight.

Thanks to office mates new and old for tea breaks, MATLAB pointers and healthy procrastination. Thanks to Shaun Eaves for scientific chats, gardening tips and beer pong glory. We have come along way since our rationed consumption of Bakewell Tarts in that Scottish Highlands hut.

To my family, I greatly appreciate the unconditional support and love you have provided along my, what may have seemed never-ending, university journey. To Juliet, thank you for keeping me smiling to the end.

However the efforts of a Ph.D. thesis are described - a science quest, a journey of selfdoubt, an odyssey of enlightenment - I have thoroughly enjoyed it. 


\section{Contents}

Abstract iii

Acknowledgements

Table of Contents

List of Figures

List of Tables

xvii

1 Introduction 1

1.1 Ice sheets, climate and sea level $\ldots \ldots \ldots \ldots$

1.2 Research focus . . . . . . . . . . . . . . 3

1.3 Thesis structure and outline . . . . . . . . . . . . . 5

1.4 Contributions made to the thesis by the author, supervisors and collaborators . . . . . . . . . . . . . 6

2 Background 9

2.1 Antarctic glaciology . . . . . . . . . . . . . . . . . 9

2.2 Past climate in Antarctica . . . . . . . . . . . . . . . . . . . . 14

2.3 Ice sheet variability in the Ross Sea sector . . . . . . . . . . . . . 17

2.3.1 Miocene, Pliocene \& Pleistocene ice sheet extents _. . . . . . 18

2.3.2 Last Glacial Maximum and subsequent deglaciation _. . . . . 19

2.4 Antarctic contribution to past sea level rise . . . . . . . . . . . . . . 23

2.5 Constraining past ice sheet behaviour f . . . . . . . . . . 27

2.6 Research questions . . . . . . . . . . . . . . . . . . 28 
3.1 In situ-produced cosmogenic nuclides . . . . . . . . . . . . . . . 29

3.1.1 Cosmic-ray flux at the Earth's surface . . . . . . . . . . . 29

3.1.2 Nuclide production in quartz . . . . . . . . . . . . . 34

3.1.3 Temporal and spatial variations in production rates . . . . . . 36

3.2 Surface exposure dating . . . . . . . . . . . . . . . . . 41

3.2.1 Cosmogenic nuclide inheritance and other sources of error . . . . 42

3.2 .2 Sampling strategy . . . . . . . . . . . . . . . 43

3.2.3 Sample preparation, geochemistry and AMS measurement . . . . 44

3.2.4 Exposure age determination . . . . . . . . . . . 45

3.3 Glaciological modelling . . . . . . . . . . . . . . . . . . 46

3.3.1 One-dimensional flowline model approach . . . . . . . . . . 46

3.3.2 Numerical model formulation . . . . . . . . . . . . . . 46

3.3.3 Boundary conditions and environmental forcing . . . . . . . . 51

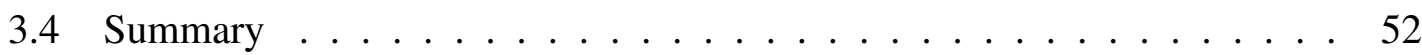

\section{Dynamics of Skelton Glacier under past} and present climates

4.1 Introduction . . . . . . . . . . . . . . . . 53

4.1.1 Previous glaciological studies of Skelton Glacier . . . . . . . . . 54

4.2 Dynamic setting as inferred from observational datasets . . . . . . . . 56

4.3 Glacier sensitivity to environmental and physical controls . . . . . . . . . . . . . . . . . . 6 60

4.3.1 Model set-up and empirical constraints . . . . . . . . . . 60

4.3.2 Simulating present-day Skelton Glacier . . . . . . . . . . . . 61 
4.3.3 Sensitivity of Skelton Glacier to environmental variables . . . . . 62

4.4 Dynamics and basal conditions of Skelton Glacier under different climates . . . . . . . . . . . . . . . . 70

4.5 Discussion ........................... 73

4.5.1 Topographically-controlled flow of Skelton Glacier . . . . . . . . 73

4.5.2 Sensitivity of Skelton Glacier to environmental forcings . . . . . 73

4.5.3 Glacier dynamics under past climates . . . . . . . . . 75

4.6 Conclusion ........................... 76

5 Complex exposure history of nunataks at Skelton Glacier since the Miocene revealed from multiple-isotope cosmogenic nuclide dating 79

5.1 Introduction . . . . . . . . . . . . . . . . 79

5.1 .1 Study area. . . . . . . . . . . . . . 80

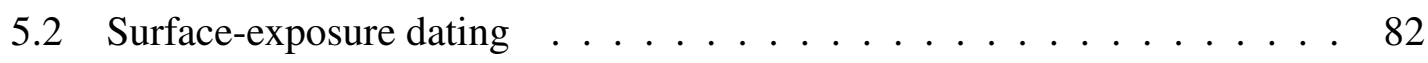

5.2.1 Method ..................... 82

5.2 .2 Results ...................... 86

5.3 Discussion ............................... 91

5.3.1 Skelton Glacier during the last glacial cycle . . . . . . . . . . . . 91

5.3.2 Long-term nuclide production rate higher than today . . . . . . . 94

5.3.3 Burial-exposure history since the Miocene . . . . . . . . . . . 96

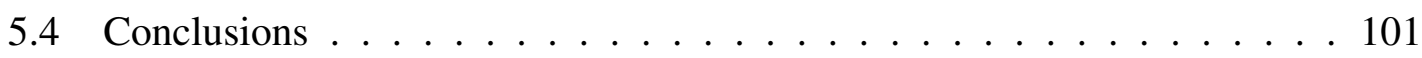

6 Long-term palaeo-dynamics of Mackay Glacier $\begin{array}{ll}\text { inferred from preserved and modified geomorphology } & 103\end{array}$

6.1 Introduction . . . . . . . . . . . . . . . . . 103

6.2 Geographic setting . . . . . . . . . . . . . . . 108 
6.3 Surficial geology and geomorphology . . . . . . . . . . . . . 108

6.3 .1 Mt Gran . . . . . . . . . . . . . . . . . . 109

6.3.2 Mt Suess \& Gondola Ridge . . . . . . . . . . . . . . . . 113

6.3.3 Low Ridge \& Cuff Cape . . . . . . . . . . . . . . . . . 116

$6.3 .4 \quad$ Kar Plateau . . . . . . . . . . . . . . . . . . 119

6.4 Discussion . . . . . . . . . . . . . . . . 121

6.4.1 Early wet-based glaciation . . . . . . . . . . . 121

6.4.2 Extent and erosion during the last glaciation . . . . . . . . . 124

6.4.3 Evidence of past flow directions . . . . . . . . . . 128

6.4.4 Cryoturbated geomorphology . . . . . . . . . . . . 129

6.5 Conclusions and wider implications . . . . . . . . . . . . . 132

\section{Rapid thinning of Mackay Glacier during} stable Holocene climate

7.1 Introduction . . . . . . . . . . . . 135

7.2 Geological evidence at Mackay Glacier . . . . . . . . . . . . . 136

7.2.1 Sample collection, age calculation and outliers . . . . . . . 137

7.2.2 Bayesian age-elevation modelling . . . . . . . . . . 141

7.2.3 Record of surface lowering at Mackay Glacier . . . . . . . . . 143

7.2.4 Regression analysis of rapid thinning . . . . . . . . . . . . 144

7.3 Stable environmental forcing during the Holocene . . . . . . . . . . . . . 147

7.4 Regional context for deglaciation as a potential cause of rapid thinning . . 148

7.4.1 Deglaciation in the western Ross Sea . . . . . . . . . . . 148

7.4.2 Mechanisms of rapid thinning at Mackay Glacier . . . . . . . . . 149 
7.5 Numerical flowline modelling of grounding-line retreat and upstream thinning . . . . . . . . . . . . . . . . 152

7.5.1 Set-up of flowline model . . . . . . . . . . . . . 152

7.5.2 Simulated thinning from grounding-line retreat $\ldots . . . . .154$

7.6 Wider empirical records of Antarctic deglaciation . . . . . . . . . . 158

7.6.1 Evidence of circum-Antarctic deglaciation . . . . . . . 158

7.6.2 Absence of significant environmental forcing at the time of rapid ice sheet thinning ................... 163

7.7 Conclusions . . . . . . . . . . . . . . . . . . . . . 164

8 Synthesis

8.1 Principal conclusions . . . . . . . . . . . . . . . . 165

8.2 Recommendations for future work . . . . . . . . . . . . . . 168

8.3 Overarching research contribution . . . . . . . . . . . . . . . 169

A Sample collection \& preparation

A.1 Field work . . . . . . . . . . . . . . . . . . 177

A.2 Sample preparation . . . . . . . . . . . . . . . . 177

B Quartz preparation and separation of cosmogenic Be and Al

B.1 Initial quartz separation . . . . . . . . . . . . . . . . . 179

B.2 Quartz cleaning and etching (HF version) . . . . . . . . . . . 179

B.3 Final Quartz Leach . . . . . . . . . . . . . . . . . . . . . 181

B.4 Carrier addition and sample dissolution f . . . . . . . . . . . 182

B.5 Dissolution . . . . . . . . . . . . . . . . 182 
B.5.1 Version 1: slow and steady . . . . . . . . . . . . . 182

B.5.2 Version 2: fast and furious ............ 183

B.6 Sample conversion . . . . . . . . . . . . . . . . . 183

B.6.1 Sample conversion (with Al-chemistry) . . . . . . . . . 183

B.6.2 $\mathrm{BeF}_{2}$ - Leaching (only when NO Al-chemistry will be done) . . . 184

B.7 Column $\mathrm{Fe} \ldots \ldots \ldots \ldots$. . . . . . . . . . . . . . 185

B.8 Column Be . . . . . . . . . . . . . . . . . . . . . 186

B.8.1 1ml Column Be (Clean Samples: $\mathrm{Al}<5 \mathrm{mg}) \ldots \ldots 186$

B.8.2 5ml Column Be (Dirty Samples: $\mathrm{Al}>5 \mathrm{mg}) \ldots \ldots$. . . . . . . 187

B.9 Column Al . . . . . . . . . . . . . . . . . . . . . 188

B.10 Precipitation . . . . . . . . . . . . . . . . . . . . 189

B.10.1 Be precipitation . . . . . . . . . . . . . . . . 189

B.10.2 Al precipitation . . . . . . . . . . . . . . 190

C Analysis of surface-exposure data 191

C.1 Complex burial-exposure history model . . . . . . . . . . . . . . 191

C.2 Regression of rapid thinning through Monte Carlo simulations . . . . . . 194 


\section{List of Figures}

1.1 Projected contribution to sea level by $2100 \mathrm{AD}$ from changes in Antarctic surface mass balance and outflow . . . . . . . . . . . . . . . . 2

1.2 Study area ......................... 5

2.1 Datasets reflecting modern ice sheet mass changes in Antarctica . . . . . 10

2.2 Antarctic ice sheet surface and bed elevations with longitudinal profiles of example outlet glaciers . . . . . . . . . . . . . . . . 13

2.3 Past changes in Antarctic climate . . . . . . . . . . . . . . . . 16

2.4 Current understanding of LGM to Holocene ice sheet history in the Ross Sea ............................ 20

2.5 Sea level contributions from Antarctica following the LGM . . . . . . . 26

3.1 Geomagnetic field and affect on incoming primary cosmic rays . . . . . 30

3.2 Cascade of secondary cosmic rays that are produced in the atmosphere and rock . . . . . . . . . . . . . . . . . . . . 31

3.3 Cosmogenic nuclide concentrations through time $\ldots \ldots 33$

3.4 Evolution of nuclide concentrations from different attenuation lengths and rock densities during continuous periods of exposure and burial . . . . . . 35

3.5 Nuclide concentrations over time with continuous exposure . . . . . . . 36

3.6 Nuclide concentrations over time with continuous exposure and steadystate erosion . . . . . . . . . . . . . . . . . . 37

3.7 Nuclide concentrations over time with exposure and burial isochrons . . . 38

3.8 Atmospheric pressure-altitude relationship in Antarctica compared to the standard atmosphere . . . . . . . . . . . . . . . . 39

3.9 Scenario of changing production rate and exposure age with altitude . . . 40

4.1 Geographic context of Skelton Glacier . . . . . . . . . . . . . . . . 55 
4.2 Features and characteristics of Skelton Glacier from observational datasets 58

4.3 Flow of Skelton Glacier from observations along the central flowline . . . 59

4.4 Simulated present-day Skelton Glacier . . . . . . . . . . . . . . 63

4.5 Test of surface elevation as a measure for changes in steady-state glacier profile ........................... 64

4.6 Sensitivity of Skelton Glacier to environmental variables . . . . . . . . . 66

4.7 Simulated along-flow sensitivity of Skelton Glacier to changes in calving $\quad 67$

4.8 Simulated along-flow sensitivity of Skelton Glacier to changes in precipitation ............................ 68

4.9 Simulated along-flow sensitivity of Skelton Glacier to changes in mean annual air temperature . . . . . . . . . . . . . . . . . 69

4.10 Simulated configurations and dynamics of Skelton Glacier under presentday, LGM, Pliocene interglacial and Pliocene glacial climates . . . . . . . 72

5.1 Overview of Skelton Glacier and nunataks targeted for surface-exposure dating ........................... 81

5.2 Samples collected at Skelton Glacier nunataks . . . . . . . . . . . . . . . 84

5.3 Consistent ${ }^{26} \mathrm{Al}$ concentrations derived from VUW and GFZ measurements 86

5.4 Probability plots of apparent ${ }^{10} \mathrm{Be}$ and ${ }^{26} \mathrm{Al}$ exposure ages at Névé Nunatak, the unnamed nunatak and Clinker Bluff . . . . . . . . . . . . . . . . 89

5.5 Two-nuclide plot showing complex sample exposure history . . . . . . . 90

5.6 Evolution of nuclide concentrations during a typical Late Pleistocene glacialinterglacial cycle . . . . . . . . . . . . . . . . . . 93

5.7 Modelled burial-exposure history over the last $5 \mathrm{Ma} \ldots \ldots$. . . . . . . 98

5.8 Modelled burial-exposure history over the last $15 \mathrm{Ma} \ldots$. . . . . . . . . 99

6.1 Location of Mackay Glacier and study sites . . . . . . . . . . . . . 105

6.2 Modelled basal conditions of Mackay Glacier at the LGM . . . . . . . 107 
6.3 Surficial geology and geomorphology at Mt Gran . . . . . . . . . . . . 110

6.4 Selected representative views of geomorphic features at Mt Gran . . . . 112

6.5 Surficial geology and geomorphology at Mt Suess and Gondola Ridge . . 114

6.6 Selected representative views of geomorphic features at Mt Suess and Gondola Ridge . . . . . . . . . . . . . . . . . . . . . . . . 115

6.7 Vertical transect of the erosional and depositional signature at Mt Suess . 116

6.8 Surficial geology and geomorphology at Low Ridge and Cuff Cape . . . . 117

6.9 Geomorphic features at Low Ridge and Cuff Cape . . . . . . . . . . . . . 118

6.10 Surficial geology and geomorphology at Kar Plateau . . . . . . . . . 119

6.11 Selected representative views of geomorphic features at Kar Plateau . . . 120

6.12 Minimum geothermal heat flux required for basal ice to reach pressure melting point as a function of ice thickness and surface temperature . . . 122

6.13 Overview of glacial geomorphology down-glacier and a reconstructed profile of Mackay Glacier at the LGM . . . . . . . . . . . . . . 126

6.14 Ground climate as soil temperature and water content at Granite Harbour, mouth of Mackay Glacier . . . . . . . . . . . . . . . . . . . . . . 130

7.1 Geographic context of Mackay Glacier . . . . . . . . . . . . . . . 136

7.2 Locations of thinning transects and photos of example samples at Mackay Glacier . . . . . . . . . . . . . . . . . . . . 138

7.3 Surface exposures ages from 4 transects at Mackay Glacier . . . . . . . 142

7.4 Age-modelled chronology of surface lowering at Mackay Glacier and regional climate forcing . . . . . . . . . . . . . . . . . . . . 144

7.5 Recorded thinning of Mackay Glacier from differing ${ }^{10}$ Be production rates 145

7.6 Regression analysis of rapid thinning at Mt Suess/Gondola Ridge (upper) and Low Ridge . . . . . . . . . . . . . . . . . . . . . . 146

7.7 Rates and durations of rapid thinning derived from the surface-exposure chronology . . . . . . . . . . . . . . . . . . . . 147 
7.8 Deglaciation in the vicinity of Mackay Glacier . . . . . . . . . . . . . 150

7.9 Staggered grounding-line retreat and reverse bed slope of Mackay Glacier

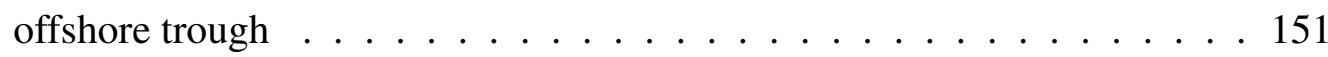

7.10 Ice surface and bed elevation estimations at Mackay Glacier . . . . . . 153

7.11 Modelled retreat of Mackay Glacier through the overdeepened trough with corresponding accelerated upstream thinning at the sample transects 155

7.12 Modelled ice flux during retreat of Mackay Glacier through an overdeepened trough . . . . . . . . . . . . . . . . . . 156

7.13 Model experiments of grounding-line retreat and surface thinning at the sample transects . . . . . . . . . . . . . . . . . . 157

7.14 Circum-Antarctic evidence of ice sheet retreat and surface lowering compared to climate changes during the last deglaciation $\ldots \ldots$. . . . . . 163

A.1 Examples of collected samples assessed for surface-exposure dating . . . 178 


\section{List of Tables}

3.1 Cosmogenic nuclides. Common nuclides and their reactions at the Earth's surface. . . . . . . . . . . . . . . . . 32

4.1 Physical constants and model parameters . . . . . . . . . . . . . . 61

4.2 Environmental parameters (modern) . . . . . . . . . . . . 62

4.3 Climate parameters used to investigate the sensitivity of Skelton Glacier . 64

4.4 Environmental parameters (past) . . . . . . . . . . . . . . . 71

5.1 Details of samples from Skelton Glacier nunataks . . . . . . . . . . . . 85

5.2 Skelton Glacier sample concentrations . . . . . . . . . . . . . . . 85

$5.3{ }^{10}$ Be apparent surface-exposure ages at Skelton Glacier . . . . . . . . . . 87

$5.4{ }^{26} \mathrm{Al}$ apparent surface-exposure ages at Skelton Glacier . . . . . . . . . . 87

7.1 Sample details and raw surface-exposure ages from Mackay Glacier, divided by altitudinal transect . . . . . . . . . . . . . . . . . . . 139

7.2 Bayesian modelled surface-exposure ages at Mackay Glacier, divided by altitudinal transect . . . . . . . . . . . . . . . . . . . . 140

7.3 Deglaciation evidence in the vicinity of Mackay Glacier . . . . . . . . . 149

7.4 Flowline model parameters . . . . . . . . . . . . . . . . . . . 154

7.5 Circum-Antarctic chronologies of West and East Antarctic grounding-line retreat . . . . . . . . . . . . . . . 160

7.6 Circum-Antarctic "dipstick" thinning transects . . . . . . . . . . . . . 161 
xviii 


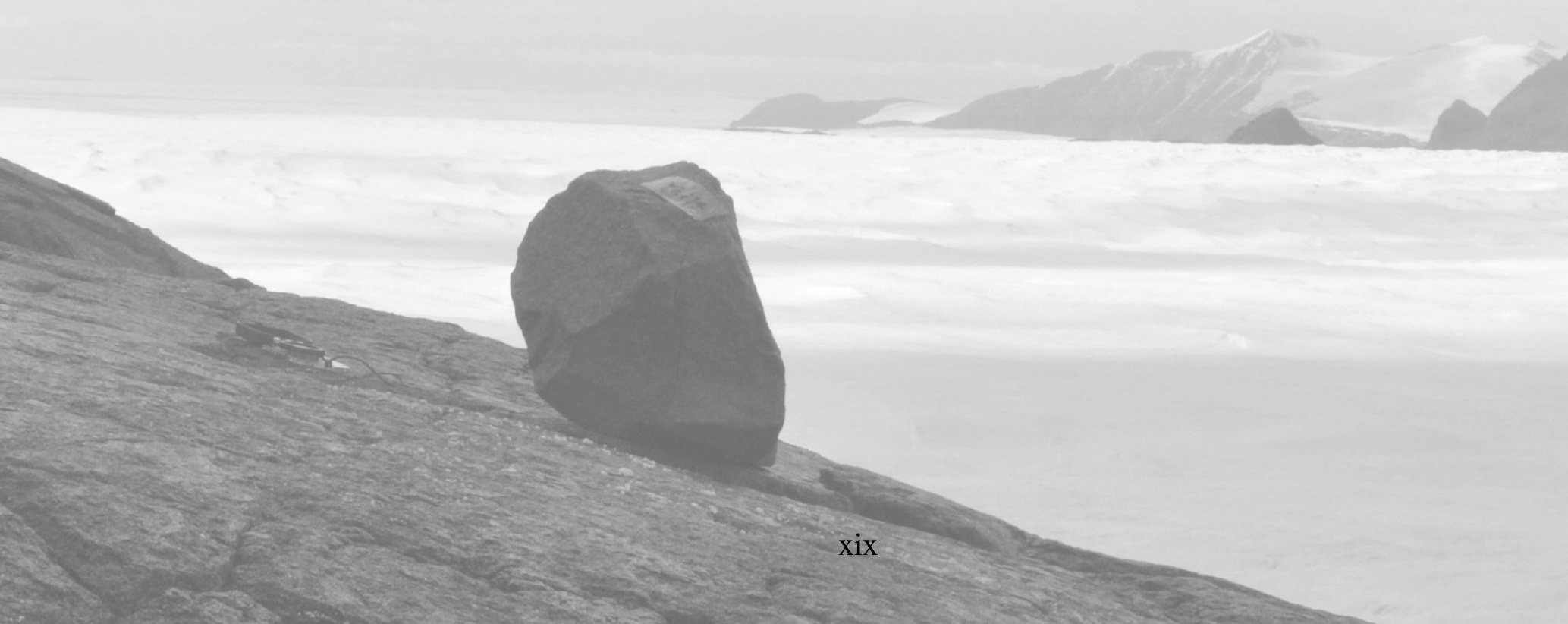




\section{Chapter 1}

\section{Introduction}

\subsection{Ice sheets, climate and sea level}

Antarctica's geological history holds insight into the key drivers and behaviour of ice sheet systems. In this thesis, I investigate the evolving configurations and dynamics of two East Antarctic outlet glaciers during the Late Cenozoic. Previous studies have helped constrain past ice sheet fluctuations using surface-exposure dating (e.g. Stone et al., 2003; Lilly et al., 2010) whereas others have improved our understanding of past and present outlet glacier dynamics using numerical modelling (e.g. Pollard and DeConto, 2009; Golledge and Levy, 2011; Jamieson et al., 2012; Favier et al., 2014; Joughin et al., 2014). Combining these approaches, I explore how the two study glaciers changed their geometry and flow regimes in different climatic states, ultimately deciphering how they respond to external forcings and internal instabilities.

The cryosphere is starting to respond to anthropogenic warming (Marzeion et al., 2014), with almost all glaciers and ice sheets worldwide having shrunk over the last century (Vaughan et al., 2013). Ice sheets in Antarctica - covering $~ 8.3 \%\left(12.295 \mathrm{Mkm}^{2}\right.$ ) of Earth's land surface and accounting for 58.3 metres of equivalent sea level (Fretwell et al., 2013; Vaughan et al., 2013) - are in the spotlight. Antarctica plays an important role in regulating global atmospheric (Russell and McGregor, 2010) and oceanic (Toggweiler and Russell, 2008) circulation, and is now showing signs of a warming climate (e.g. Steig et al., 2009). Satellite observations reveal that some outlet glaciers in Antarctica are experiencing decadal-scale dynamic thinning, terminus retreat and mass loss (Miles et al., 2013; Pritchard et al., 2009; Shepherd et al., 2012). However, the extent to which this behaviour occurs beyond natural variability remains unclear. Enhanced outflow in West Antarctica (Rignot et al., 2011) is currently considered to outweigh increased accumulation in East Antarctica (Lenaerts et al., 2012; Harig and Simons, 2015), suggesting that Antarctic ice sheets will contribute to sea level rise in the coming decades (Church et al., 2013) (Figure 1.1). However, predictions of future sea level change are dependent on the current state of knowledge of ice sheet behaviour (Bindschadler et al., 2013), and while numerical modelling efforts have improved our understanding of the processes driving observed ice sheet changes (e.g. Favier et al., 2014), predictions of the long-term responses have large uncertainties (e.g. Joughin et al., 2014; Mengel and Levermann, 2014). 


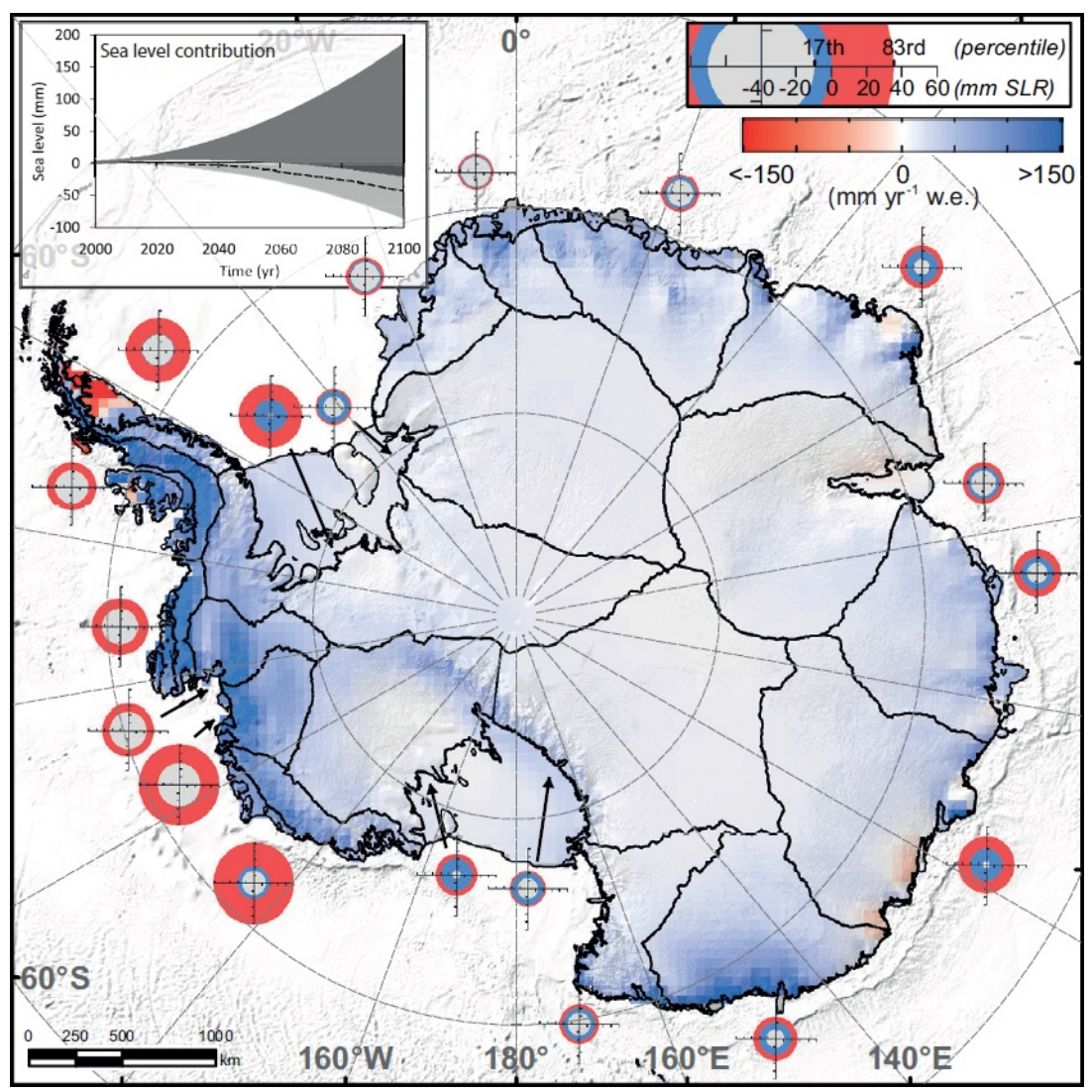

Figure 1.1 Projected contribution to sea level by 2100 AD from changes in Antarctic surface mass balance and outflow (after Church et al., 2013). Under future warming (IPCC scenario A1B), the RACMO2 regional atmospheric climate model simulates increased surface mass balance (apparent as a predominantly blue ice surface), however mass loss in Antarctica occurs primarily through increased outflow. This is shown by

coloured rings, based on probabilistic extrapolation of observed trends, where red signifies ice loss (in $\mathrm{mm}$ of sea level rise). The width, from the inner grey circle to the outer rim of the red ring, indicates the uncertainty associated with these estimates, which is particularly high in West Antarctica where non-linear dynamic responses are likely. Corresponding sea level contributions highlight how projections of enhanced outflow (dark grey) are likely to dominate the contrasting effects of positive surface mass balance (light grey). Black lines denote the grounding-line, ice-shelf edge and drainage basins.

Geological data can extend the record of ice sheet observations, helping to demonstrate the sensitivity of ice sheets to climate forcing and elucidate the processes driving presentday and future ice sheet responses. Reconstructing the glacial history of Antarctica contributes to our understanding by; 1) improving modern satellite-derived mass balance estimates, 2) constraining the source and nature of past sea level rise episodes, and 3) identifying ice sheet responses on centennial to millennial timescales. These will now be briefly discussed.

Changes in modern ice sheet mass balance are estimated in part through satellite-derived gravity surveys (GRACE), however, the precision of these estimates is hampered by uncertainties in isostatic uplift (e.g. King et al., 2012), which results from the lagged 
response of Earth's crust to deglaciation following the Last Glacial Maximum (LGM) 25-18 ka ago (Clark et al., 2009). Glacio-isostatic adjustment (GIA) models are used to determine uplift rates, constrained by geological data that record the deglacial history of the Antarctic continent (e.g. Ivins and James, 2005; Whitehouse et al., 2012). Insufficient records currently exist from the Antarctic margin and, therefore, further constraints on the LGM-to-recent glacial history are crucial for refining modern mass balance estimates.

Global eustatic sea level rise following the LGM was not a gradual progression (e.g. Lambeck et al., 2014), and instead occurred with distinct pulses associated with the melt of ice sheets (e.g. Clark et al., 2002; Carlson and Clark, 2012). Recent offshore data of past iceberg rafting events indicate several potential pulses of ice loss from Antarctica (Weber et al., 2014), and such ice loss has been simulated with an ice sheet model (Golledge et al., 2014b). However, this is not supported by available onshore evidence (e.g. Mackintosh et al., 2007; Bentley et al., 2010). The volume of ice loss during this time is not well constrained, and additional geological data are required from Antarctica to record both maximum surface elevations at the LGM and the subsequent onset and rate of surface lowering.

Sedimentological records indicate that the West and East Antarctic Ice Sheets fluctuated over the last $5 \mathrm{Ma}$ in response to an orbitally-driven climate (Naish et al., 2009; Patterson et al., 2014). During warm periods, deglaciation may have led to partial ice sheet collapse (Pollard and DeConto, 2009; Cook et al., 2013). Unfortunately, the paucity, resolution and imperfect preservation of these records prohibits adequate determination of the rates of ice sheet retreat or ice surface lowering during these events. Furthermore, the ice dynamics during past deglaciation periods and past steady-state climates remain poorly understood. These uncertainties limit the prediction of multi-century sea level contributions from Antarctica (Church et al., 2013).

\subsection{Research focus}

In this thesis, the glacial history of a sector of Victoria Land is reconstructed for the Late Cenozoic. The time from the LGM to present-day is a particular focus as it represents the last major period of carbon dioxide increase and atmospheric warming, providing an opportunity to better understand the response of ice sheets to enhanced warming. Antarctica's cold climate allows for the preservation of a much longer ice sheet history in the landscape record, and therefore this work also explores earlier Pliocene and Pleistocene ice sheet fluctuations and dynamics, at a time when the climate was similar to, and warmer than, today. 
Central to this study is the application of surface-exposure dating of glacially-transported cobbles and glacially-scoured bedrock surfaces in order to constrain past ice sheet history. This approach exploits the accumulation of rare isotopes $\left({ }^{10} \mathrm{Be},{ }^{26} \mathrm{Al}\right)$ at the Earth's surface, produced as a result of the continuous bombardment of cosmic radiation. In this way, the time since a rock was exposed during deglaciation can be determined with known rates of radionuclide production and decay. Numerical flowline modelling is used to complement these geological data, allowing for the investigation of controls on surface lowering through idealised experiments.

My research focuses on two East Antarctic outlet glaciers that flow through the Transantarctic Mountains to the Ross Sea embayment; Skelton Glacier and Mackay Glacier, south and north of the Dry Valleys (Figure 1.2). These glaciers are important study areas for several reasons: 1) the glacial history determined here is supported by numerous onshore and offshore records of ice sheet and ice-shelf advance and retreat located in the vicinity (e.g. Naish et al., 2009; Anderson et al., 2014); 2) Transantarctic Mountain outlet glaciers were likely buttressed by an expanded West Antarctic Ice Sheet during glacial periods (e.g. Bockheim et al., 1989; Conway et al., 1999), and therefore changes in the geometry of these glaciers are anticipated to represent fluctuations of the adjacent grounded ice sheet; 3) glacial overdeepenings exist downstream of the grounding-lines of Skelton and Mackay Glaciers, suggesting the potential for topographically-driven instability (e.g. Weertman, 1974); 4) previous glaciological and climatological studies (e.g. Crary, 1966; Calkin, 1974; Steig et al., 1998) help constrain the boundary conditions for numerical modelling experiments; and 5) Skelton Glacier and Mackay Glacier represent contrasting settings, flowing into the Ross Ice Shelf, and open water respectively.

The research documented in this thesis is based around a series of overarching themes. Specifically, I focus on;

- Changes in the geometry and dynamics of Transantarctic Mountain outlet glaciers during the Pliocene and Quaternary

- Ice sheet thickness in the western Ross Sea at the Last Glacial Maximum, and possible contribution to 'meltwater pulses'

- Onset of ice surface lowering following the Last Glacial Maximum and the dynamic processes facilitating deglaciation

- Sensitivity of Transantarctic Mountain outlet glaciers to environmental forcing and topographic feedbacks

- Implications for observed and projected ice sheet changes 


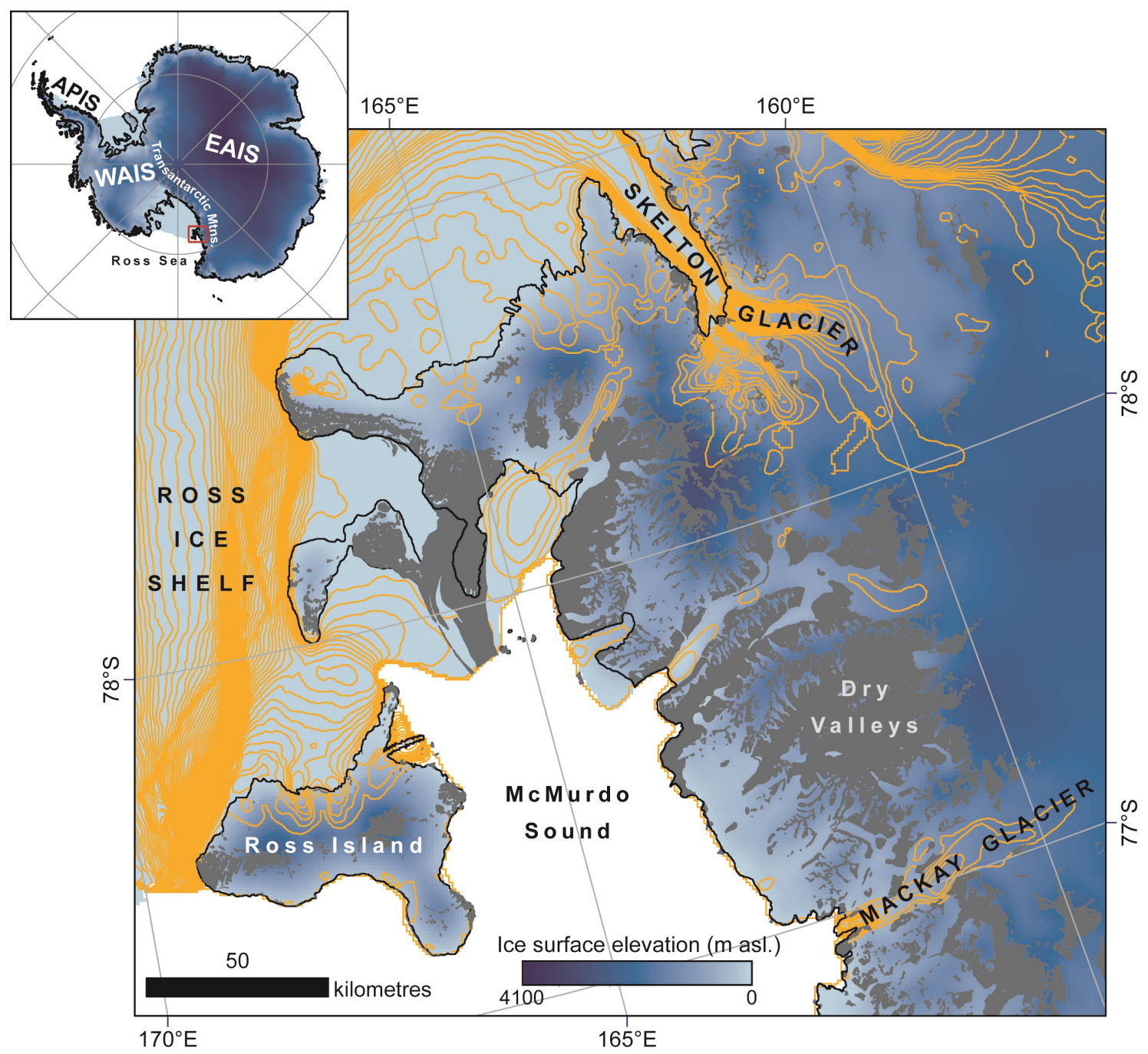

Figure 1.2 Study area. The inset shows the West Antarctic (WAIS), East Antarctic (EAIS) and Antarctic Peninsula (APIS) Ice Sheets, and the location of the main figure as a red box. Ice surface velocity contours (orange, in $10 \mathrm{~m} \mathrm{a}^{-1}$ intervals) highlight the main flow paths of ice in this area (Rignot et al., 2011). Skelton and Mackay Glaciers flow from Taylor Dome at the edge of the East Antarctic polar plateau, through the Transantarctic Mountains, to the Ross Ice Shelf and Ross Sea respectively. Exposed rock and drift deposits are shown in grey. The modern grounding-line is represented by a black line. Ice surface elevations are from Fretwell et al. (2013).

\subsection{Thesis structure and outline}

This thesis has been formatted in a traditional style; it contains an introduction, background, methodology, a series of central research chapters, and an overarching synthesis with conclusions. In Chapter 2, the background provides the context and rationale to my work. Our current knowledge of Antarctic ice sheets, palaeoclimate, glacial history and scientific developments in understanding these topics is discussed. Chapter 3 outlines the basis of surface-exposure dating and numerical modelling approaches, and describes how the techniques are applied here. 
The main chapters (Chapters 4-7) are self-contained scientific studies, which have been submitted, or will be submitted in a similar form, to international journals. While their inclusion attempts to maintain a coherent structure within the thesis, some repetition does occur (e.g. study site descriptions, sampling and modelling approaches). Chapter 4 investigates the past and present glaciology of a Transantarctic Mountain outlet glacier, achieved through numerical modelling of Skelton Glacier. In Chapter 5, surface-exposure data is presented from Skelton Glacier, building on interpretations from modelling in the previous chapter to reveal long-term ice sheet fluctuations over the Late Cenozoic. Chapter 6 investigates the past glaciology of a Transantarctic Mountain outlet glacier, on this occasion through geomorphological assessment of Mackay Glacier. The deglaciation history and dynamics from the LGM to present-day are then comprehensively studied for Mackay Glacier using surface-exposure dating and numerical modelling in Chapter 7.

The outcomes and implications of this research are summarised and discussed in a final synthesis (Chapter 8). Here, responses are provided for each of the research questions (introduced in Chapter 2.6). The details of sample collection and cosmogenic nuclide $\left({ }^{10} \mathrm{Be}\right.$, ${ }^{26} \mathrm{Al}$ ) processing procedures used in Chapters 5 and 7 are outlined in Appendix A and $\mathrm{B}$, while the MATLAB scripts developed to model burial-exposure histories in Chapter 5 and perform regression analysis of glacier thinning transects in Chapter 7 are presented in Appendix C.

\subsection{Contributions made to the thesis by the author, supervisors and collaborators}

Research in this thesis was designed, carried out, interpreted and written up by the author, following an idea originally envisioned by primary supervisor A. Mackintosh (Victoria University of Wellington (VUW)). Samples for surface-exposure dating were collected with the help of G. Wilson (University of Otago) at Skelton Glacier and C. Fogwill (University of New South Wales, Australia) and K. Norton (VUW) at Mackay Glacier. Sample processing and isotope extraction were carried out by myself at Victoria University of Wellington and GNS Science, with supervision from K. Norton. Measurements of ${ }^{10} \mathrm{Be}$ and ${ }^{26} \mathrm{Al}$ were made by P. Kubik, M. Christl and C. Vockenhuber (ETH Zurich, Switzerland), while measurements of stable $\mathrm{Al}$ were carried out by myself and validated by $\mathrm{H}$. Wittmann (GFZ Potsdam, Germany). The 1-dimensional glacier flowline model was originally coded in Python by N. Golledge (VUW and GNS Science). The model code was adapted here to suit glacier-specific conditions by N. Golledge and myself. A Monte Carlo regression model used for determining rates and durations of glacier thinning (Chapter 7) was developed together with E. Smith (VUW). 
My supervisors, A. Mackintosh, K. Norton and N. Golledge, oversaw sample processing and glacier modelling, contributed to the discussion of scientific ideas and provided editorial input for the thesis and manuscript texts.

One paper manuscript was written simultaneously with the thesis, corresponding to research presented in Chapter 7:

Jones, R.S., Mackintosh, A.N., Norton, K.P., Golledge, N.R., Fogwill, C.J., Kubik, P.W., Christl, M. and Greenwood, S.L. Rapid Holocene thinning of an East Antarctic outlet glacier driven by marine ice sheet instability, Nature Communications (accepted).

Chapter 7 reflects an early version of this paper, at a stage of editorial input comparable to other thesis chapters. As mentioned above, co-authors C. Fogwill assisted with sample collection, P. Kubik and M. Christl carried out AMS measurements and S. Greenwood (Stockholm University, Sweden) provided discussion on the regional deglaciation context.

Clarification should also be made regarding a recently published paper led by N. Golledge:

Nicholas R. Golledge, Oliver J. Marsh, Wolfgang Rack, David Braaten, R. Selwyn Jones (2014), Basal conditions of two Transantarctic Mountain outlet glaciers from observationconstrained diagnostic modelling, Journal of Glaciology, 60(223), 855-866.

This study is similar to modelling efforts in Chapter 4. While some outcomes overlap, the studies are independent. Golledge et al. (2014a) applied a diagnostic modelling approach, focused on exploiting high-quality empirical radar and velocity data in an iterative method, to derive the present-day dynamics of Skelton Glacier. The work here instead focuses on the along-flow sensitivity and time-dependent dynamics of Skelton Glacier, using a prognostic approach. 


\section{Chapter 2}

\section{Background}

Our understanding of Antarctic ice sheet behaviour has greatly improved in recent decades, however, further advances are required to fully recognise how ice sheets respond to climate change and how this response is affected by internal processes. In this chapter, I outline recent developments in scientific knowledge and current uncertainties regarding: 1) modern Antarctic glaciology, 2) past climate in Antarctica, 3) past ice sheet variability in the Ross Sea sector, 4) the Antarctic contribution to past sea level rise, and 5) approaches that can be used to better constrain past ice sheet behaviour. This discussion leads into a final section where I present the Research Questions that are addressed in this thesis.

\subsection{Antarctic glaciology}

Recent observed changes in the geometry and flow of the Antarctic ice sheet (Vaughan et al., 2013) provides motivation for better understanding its past behaviour. Large improvements in the quality and coverage of satellite, airborne and in situ measurements as well as in regional climate models have allowed for the quantification of modern Antarctic ice sheet thickness (Fretwell et al., 2013), accumulation and ablation (e.g. van den Broeke et al., 2006), ice surface velocity (Rignot et al., 2011), surface elevation changes (Pritchard et al., 2009) and volume change (e.g. Velicogna, 2009) (Figure 2.1).

Continent-wide summary datasets provide a snapshot of the mass balance and flow of the Antarctic ice sheet, as well as recent changes (Figure 2.1). For example, regional atmospheric climate models show that accumulation is greatest in the Antarctica Peninsula (>1500 $\left.\mathrm{mm} \mathrm{a}^{-1}\right)$ and in coastal West Antarctica $\left(>1000 \mathrm{~mm} \mathrm{a}^{-1}\right)$ (van den Broeke et al., 2006; Lenaerts et al., 2012). Such accumulation values lead to a positive ice sheet surface mass balance, with ablation restricted primarily to snowfall sublimation (Lenaerts et al., 2012). Mass loss in Antarctica instead mainly occurs through outflow, from subshelf melt and calving at the terminus. Satellite-derived radar interferometry reveals the spatial pattern of ice sheet flow and provides estimates of ice surface velocities (Rignot et al., 2011); flow increases exponentially from the interior (at $\mathrm{cm}$ per year scale) to the terminus (up to km per year on fast glaciers and ice shelves) as the ice surface steepens and as streaming flow becomes more prevalent. The highest velocities are observed on 

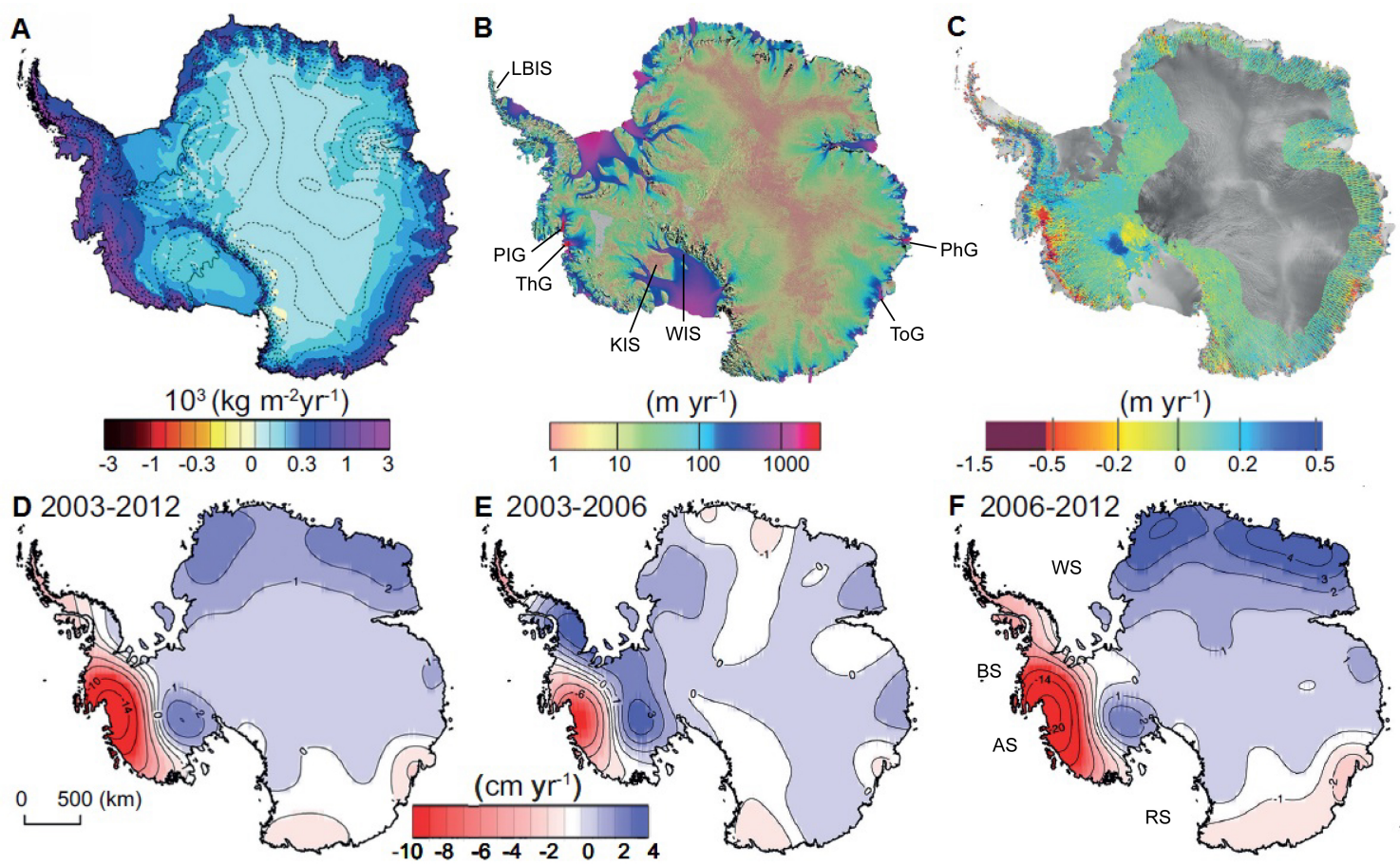

Figure 2.1 Datasets reflecting modern ice sheet mass changes in Antarctica (after Vaughan et al., 2013). A) Mean surface mass balance (1989-2004) from regional atmospheric climate modelling (van den Broeke et al., 2006). B) Ice sheet velocity (2007-2009) determined from radar interferometry (Rignot et al., 2011). C) Changes in ice sheet surface elevation (2003-2008) determined from ICESat altimetry (Pritchard et al., 2009). D-F) Temporal evolution of ice loss determined from GRACE time-variable gravity (for 2003-2012, 2003-2006 and 2006-2012, respectively) (Velicogna, 2009). Importantly, mass loss is estimated where rapid surface lowering is observed, in Pine Island and Thwaites Glaciers in West Antarctica and Totten Glacier in

East Antarctica. Labels correspond to Larsen-B Ice Shelf (LBIS), Philippi Glacier $(\mathrm{PhG})$, Totten Glacier (ToG), Whillans Ice Stream (WIS), Kamb Ice Stream (KIS), Thwaites Glacier (ThG), Pine Island Glacier (PIG), Weddell Sea (WS), Ross Sea (RS), Amundsen Sea (AS) and Bellinghausen Sea (BS).

large outlet glacier and ice streams, which are warm-based and which tend to flow over deformable sediments. In contrast, cold-based ice exists in high relief areas where ice is thinner and/or slower flowing (Pattyn, 2010).

The fastest flowing outlets are also the most rapidly changing areas of Antarctica. In particular, Pine Island Glacier and Thwaites Glacier of the West Antarctic Ice Sheet (WAIS), the fastest flowing Antarctic outlets, are currently thinning substantially at $\sim 6 \mathrm{~m} \mathrm{a}^{-1}$ (Pritchard et al., 2009). These measurements of surface lowering, obtained from repeat satellite laser altimetry, also reveal dynamic thinning of outlets of the East Antarctic Ice Sheet (EAIS) (such as Totten Glacier at $\sim 2 \mathrm{~m} \mathrm{a}^{-1}$ ), and contrasting changes in neighbouring Kamb (thickening at $\sim 0.6 \mathrm{~m} \mathrm{a}^{-1}$ ) and Whillans (thinning at $\sim 0.25 \mathrm{~m} \mathrm{a}^{-1}$ ) Ice Streams (Pritchard et al., 2009). However, the short duration of these observations (5 years, 2003-2007) precludes the determination of longer-term trends. 
Changes in ice sheet surface elevation are observable in the magnitude and spatial pattern of Antarctic mass balance estimates. Large mass loss is estimated for the WAIS (particularly around the Amundsen and Bellinghausen Seas) and the Antarctic Peninsula Ice Sheet (APIS), with additional loss from Totten, Cook and Philippi Glaciers in East Antarctica. This trend is partly countered by possible mass gain in the Weddell Sea and Ross Sea embayments. Overall, the loss has likely increased from 30 (-37 to 97) $\mathrm{Gt} \mathrm{a}^{-1}$ (1992-2001) to 147 (72 to 221) Gt a ${ }^{-1}$ (2002-2011) (Vaughan et al., 2013). However, a clear signal of modern Antarctic mass balance is limited by uncertainties in these estimates (Shepherd et al., 2012), whether derived from GRACE gravity measurements (dependent on glacioisostatic adjustment (GIA) models) (e.g. Chen et al., 2009; King et al., 2012; Velicogna, 2009) or mass budget estimates (dependent on measurements of surface mass balance and outflow) (e.g. Rignot et al., 2008). With respect to GIA mass balance corrections, significant improvements can be made by increasing constraints on the magnitude and timing of crustal unloading, primarily achieved through geological data that record Antarctica's deglaciation history (e.g. Whitehouse et al., 2012).

To better understand modern and future glaciological changes in Antarctica, research has focused on the response of ice sheets to climate and the controls of ice sheet flow, each of which will now be discussed. The largest observed changes are in the WAIS and APIS, where the ice surface lies at much lower average elevations than in East Antarctica (Fretwell et al., 2013), and these areas are therefore more sensitive to climate change (e.g. Steig et al., 2009; Mulvaney et al., 2012). Despite increasing accumulation on the Antarctic Peninsula (Thomas et al., 2008), resulting ice gain is insufficient to offset ice loss (Ivins et al., 2011). More specifically, changes from atmospheric-warming-induced melt are more significant than changes from increased precipitation in Antarctic Peninsula glaciers (Davies et al., 2014). Furthermore, increased accumulation in West Antarctica may act to enhance mass loss through steepening the surface slope and therefore increasing outflow (Winkelmann et al., 2012). Unfortunately, few studies have explored the sensitivity of Antarctic outlet glaciers to climate change and the resulting effects on basal conditions and ice discharge. This restricts our current understanding of potential ice sheet response to just a few outlet systems.

Processes occurring at the bed, margins and terminus of outlet glaciers play a significant role in stabilising ice sheets. In particular, the WAIS has the largest percentage of its area grounded below sea level (Fretwell et al., 2013) and is theorised to be vulnerable to collapse from 'Marine Ice Sheet Instability' (Weertman, 1974; Mercer, 1978; Joughin and Alley, 2011). Grounding-lines located on reverse bed slopes are potentially susceptible to this instability, where initial retreat into deeper water can lead to thicker ice at the grounding-line that is closer to floatation and therefore increased ice flux and glacier thinning, resulting in further grounding-line retreat, drawdown of ice and mass loss (Schoof, 
2007). Today, those glaciers with grounding-lines located on reverse bed slopes below sea level are also those showing rapid surface lowering and mass loss (e.g. Pine Island Glacier, Totten Glacier; Figure 2.2) (Pritchard et al., 2009; Shepherd et al., 2012).

The effectiveness of 'marine ice sheet instability' is dependent on other controls at the bed and margins. For example, Kamb Ice Stream on the Siple Coast stagnated $\sim 150$ years ago despite being grounded below sea level. Such behaviour stimulated research into the drivers and limiting factors of ice sheet discharge (e.g. Conway et al., 2002); in situ geophysical measurements and modelling experiments have helped recognise and quantify the roles of till deformation to facilitate streaming flow (e.g. Alley et al., 1986) and meltwater to maintain flow (Anandakrishnan et al., 2001) and cause short-term rapid accelerations in discharge (Stearns et al., 2008). Furthermore, idealised modelling experiments have identified the potential for outlet glacier stabilisation from enhanced lateral drag; grounding-lines can stabilise on a reverse bed slope from small variations in valley (or ice stream) width (Nick et al., 2010; Jamieson et al., 2012), with greatest effect in narrow troughs (O'Neel et al., 2005). On this basis, topographically-constrained outlet glaciers with overdeepened basins that extend below sea level, such as those in the Transantarctic Mountains (Riger-Kusk, 2011), may or may not be susceptible to rapid retreat and surface lowering.

Outlet glacier discharge is also heavily influenced by the presence or absence of a floating ice-shelf beyond the grounding-line. An ice-shelf provides enhanced lateral drag, which acts to buttress the outflow of ice from upstream. Where ocean warming occurs today (Payne et al., 2004; Jacobs et al., 2011), ice shelves thin (Pritchard et al., 2012; Depoorter et al., 2013; Rignot et al., 2013) and therefore reduce this buttressing effect. In the event of ice-shelf collapse, such as that of Larsen-B Ice Shelf, tributary glaciers accelerate and dynamically thin upstream (Scambos et al., 2004; Rignot et al., 2004). By reconstructing glacier extents at times prior to observations, we can better understand the longer-term effects of ice-shelf and associated grounding-line induced instability (e.g. Rebesco et al., 2014).

Numerical modelling efforts, incorporating many of the discussed controls on ice flow, seek to replicate observed behaviour and elucidate the future response of ice sheet outlets. Such glacier simulations predict irreversible retreat for dynamic parts of the WAIS (e.g. Pine Island and Thwaites Glaciers) (Favier et al., 2014; Joughin et al., 2014) and for large overdeepened basins of East Antarctica (Mengel and Levermann, 2014). However, simulated ice sheet loss has uncertain rates, durations and magnitudes of mass loss (Bindschadler et al., 2013).

In the Transantarctic Mountains, the dynamic response of outlet glaciers could potentially be modulated by bed overdeepenings, valley topography and/or the Ross Ice Shelf, 

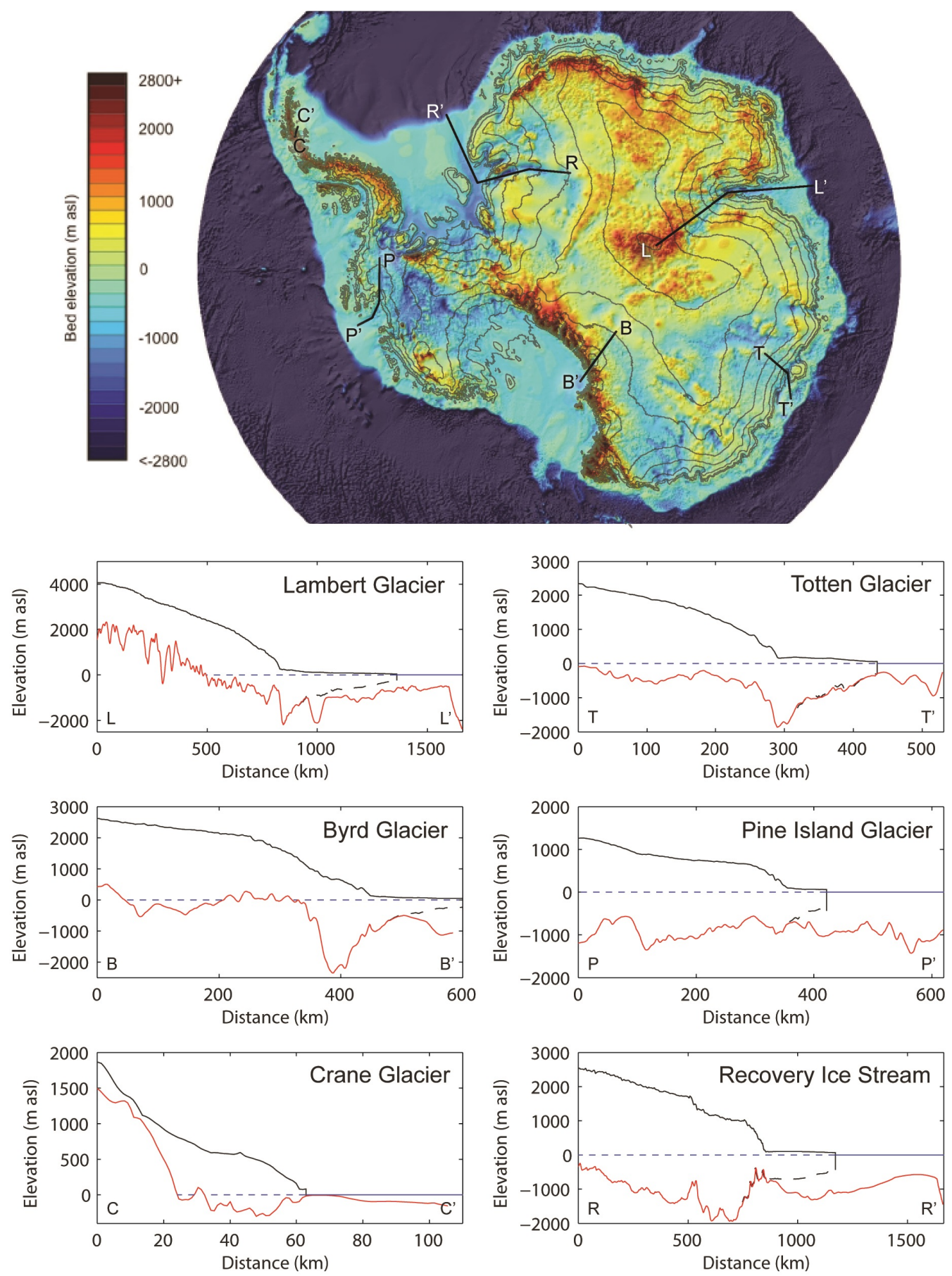

Figure 2.2 Antarctic ice sheet surface and bed elevations with longitudinal profiles of example outlet glaciers. The top panel demonstrates the substantial area of Antarctica with an ice sheet bed resting below sea level (after Fretwell et al., 2013). Ice surface elevation contours (at $500 \mathrm{~m}$ intervals) and transects along the flowlines of outlet glaciers are also shown. These transects correspond to the profiles in the lower panels, which reveal the varied surface (black) and bed (red) topography, and highlight the occurrence of overdeepened basins with reverse bed slopes near present-day grounding-lines. In particular, Totten Glacier and Pine Island Glacier are currently experiencing rapid surface lowering, which may be driven by 'marine ice sheet instability' (see text for discussion). Byrd Glacier is typical of many Transantarctic Mountain outlets, with large overdeepenings and modern ice-shelf buttressing. 
motivating further investigation. This region of Antarctica experienced an increase of glaciological research during the International Geophysical Year (1957-59), when several field campaigns obtained in situ measurements related to surface mass balance and glacier flow (e.g. Swithinbank, 1963; Giovinetto et al., 1964; Wilson and Crary, 1961). Later advances came from modelling studies, recognising the role of undulating topography on ice flux adjustment (Kavanaugh et al., 2009; Golledge and Levy, 2011) and its affect on basal ice temperature (Kavanaugh and Cuffey, 2009; Golledge et al., 2014a).

The future behaviour of Transantarctic Mountain outlet glaciers is uncertain. In recent decades, many glaciers in this sector of the EAIS have experienced marginally positive mass balances (Stearns, 2011; Rignot et al., 2008, 13 $\pm 13 \mathrm{Gt} \mathrm{a}^{-1}$ ) and advances of their termini (Miles et al., 2013). However, this trend may only reflect the short duration of satellite observations. Glacier velocities in this region can change on daily to centennial timescales (e.g. Zoet et al., 2012; Hulbe and Fahnestock, 2007), and the ice front position and mass balance of these glaciers have shown cyclic variations over at least the last 50 years (Frezzotti, 1997). It is therefore apparent that the transient behaviour of Transantarctic Mountain outlet glaciers on greater than decadal timescales is insufficiently understood.

In summary, improvements in Antarctic observations and modelling of glaciological processes have increased our understanding of ice sheet behaviour in recent decades (Hanna et al., 2013; Vaughan et al., 2013). However there are still uncertainties regarding: 1) whether the observed changes are within the range of natural variability; 2) the sensitivity of different types of outlet glacier (e.g. marine-terminating, ice-shelf-terminating, valleyconstrained) to external forcing and the extent to which ice dynamics are modulated by internal mechanisms; and 3) the timing, duration, magnitude and style of ice sheet response following an initiated instability. This thesis seeks to better understand these uncertainties, which are considered a significant limitation to the prediction of multi-century future sea level rise (Church et al., 2013). Focusing on Transantarctic Mountain outlet glaciers, past changes in ice geometry and controls on ice dynamics will be explored through numerical modelling (associated with Research Questions 1 and 4).

\subsection{Past climate in Antarctica}

The state of Antarctica's ice sheets is inherently linked to climate (e.g. Huybers and Denton, 2008; Huybers, 2009; Hanna et al., 2013). Understanding past configurations and behaviour of ice sheets therefore requires consideration of past changes in Antarctic climate. 
The Late Cenozoic was characterised by a cooling trend towards the polar climate of today, inferred from deep sea proxy records of combined ocean temperature and global ice volume (Zachos et al., 2001). This trend became more prominent during the Mid-Miocene ( $\sim 14 \mathrm{Ma}$ ), recorded as a 6-7 ${ }^{\circ} \mathrm{C}$ cooling in the Southern Ocean (Shevenell et al., 2004) and $>8{ }^{\circ} \mathrm{C}$ cooling in the terrestrial, high elevation, Transantarctic Mountains (Lewis et al., 2008). Decreases in Antarctic temperature were probably caused by low summer insolation associated with a change in orbital forcing, and decreasing $\mathrm{CO}_{2}$ (Holbourn et al., 2005). Although cooler than the Miocene, Antarctica was still warmer and wetter than today during the Pliocene (Fedorov et al., 2013; Haywood et al., 2013), which is reflected in the western Ross Sea by reconstructed sea surface temperatures of $4.5-6.5^{\circ} \mathrm{C}$ higher than present (Winter et al., 2010). Following early-mid Pliocene Antarctic warming (Escutia et al., 2009), cooling resumed in the Late Pliocene and continued into the Pleistocene, recorded in multi-proxy sea surface temperature and sea ice reconstructions from the western Ross Sea (McKay et al., 2012; Sjunneskog and Winter, 2012).

Variations in Antarctic and global palaeoclimate are best understood during the Pleistocene, with ice cores able to record continuous and centennial-scale atmospheric changes (e.g. Marcott et al., 2014). EPICA Dome C in East Antarctica currently provides the longest ice core record, extending back to $\sim 800 \mathrm{ka}$ (Jouzel et al., 2007). Using changes in $\delta^{18} \mathrm{O}$ and $\delta \mathrm{D}$ as proxies for variations in atmospheric temperature, the climate is shown to have fluctuated between glacial and interglacial states since at least the mid-Pleistocene (Figure 2.3A). These fluctuations are characterised by relatively rapid transitions from glacial to interglacial periods and then slower transitions into more prolonged glacial periods, which mirrors offshore records of ocean temperature and ice volume over this period (Lisiecki and Raymo, 2005; Huybers, 2009). The same pattern is also recorded in West Antarctica (WAIS Divide Project Members, 2013) and around the Ross Embayment, at Siple Dome (Brook et al., 2005), Talos Dome (Stenni et al., 2011) and at the divide of Skelton and Mackay Glaciers at Taylor Dome (Steig et al., 1998). Atmospheric temperature covaries with $\mathrm{CO}_{2}$ over this time (Parrenin et al., 2013), and recent high resolution records reveal a temperature lag of several centuries following changes in $\mathrm{CO}_{2}$ (Marcott et al., 2014; Shakun et al., 2012).

Ice core proxies also record changes in accumulation and wind strength, which suggest Antarctica experienced significantly reduced precipitation and more vigorous atmospheric circulation during glacial periods (e.g. Petit et al., 1999; WAIS Divide Project Members, 2013). This is recorded at Taylor Dome during the last glacial, when modified synoptic patterns and negligible accumulation $\left(<0.1 \mathrm{~cm} \mathrm{a}^{-1}\right.$ ice equiv. $)$ accompanied atmospheric temperatures 9-10 ${ }^{\circ} \mathrm{C}$ cooler than today (Steig et al., 1998; Morse et al., 1998). Subsequent warming is shown from $\sim 20-18 \mathrm{ka}$ in both Antarctic ice core proxies (WAIS 


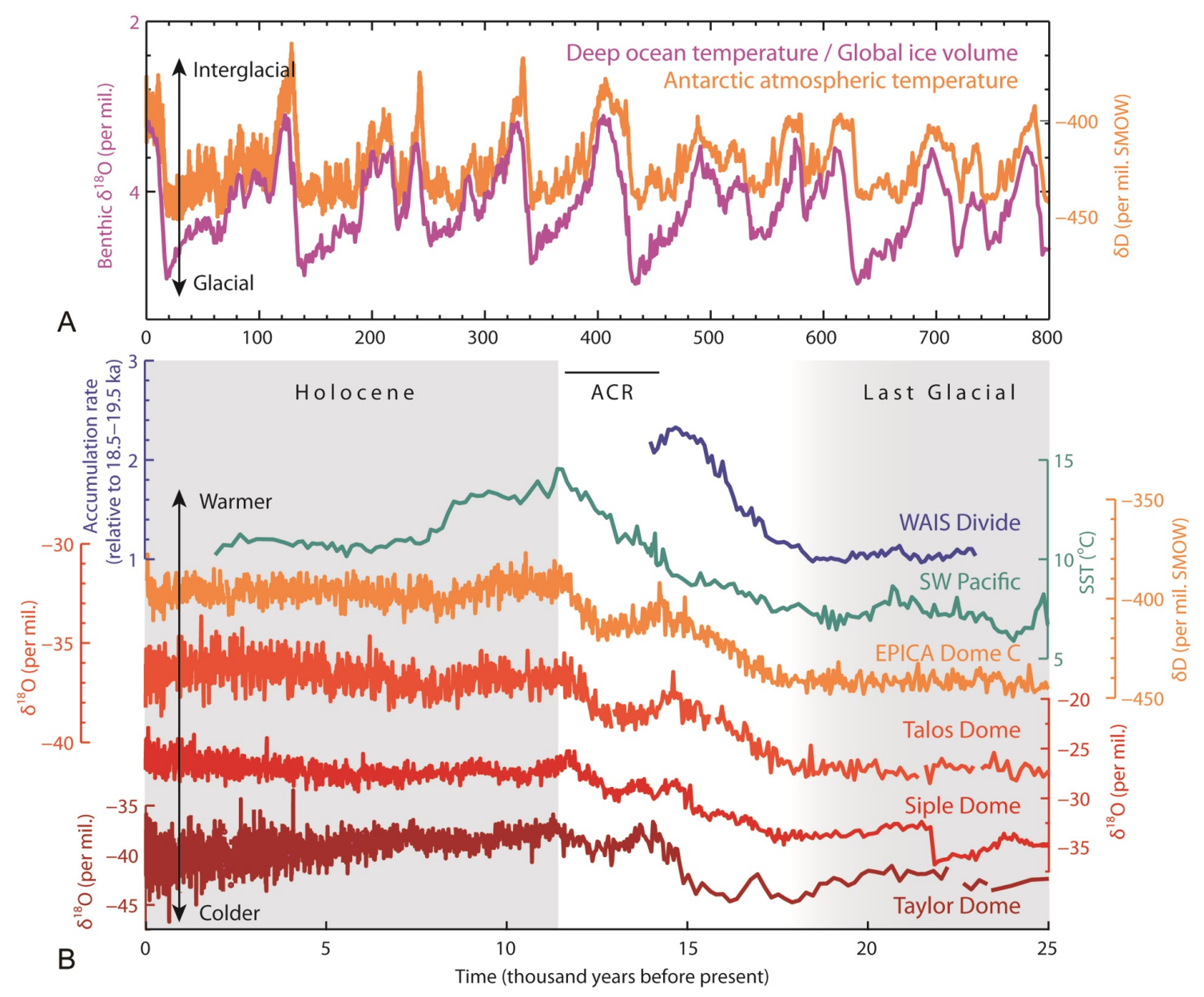

Figure 2.3 Past changes in Antarctic climate. A) The last $800 \mathrm{ka}$ of fluctuating atmospheric temperature in East Antarctica, inferred from $\delta \mathrm{D}$ from an ice core (EPICA Dome C; Jouzel et al., 2007), compared to inferred changes in deep ocean temperature and global ice volume (Lisiecki and Raymo, 2005). Interglacial periods were relatively short with long transitions into prolonged glacial periods that then terminated relatively rapidly. B) Antarctic climate through the last $25 \mathrm{ka}$, representing the last glacial and subsequent termination to present-day. Sea surface temperatures (SST) in the south-west Pacific Ocean increased after $\sim 20 \mathrm{ka}$, peaking at $\sim 11 \mathrm{ka}$ before reducing and stabilising during the Holocene (Pahnke and Sachs, 2006). Changes in East Antarctic temperature (EPICA Dome C) also occurred in the Ross Embayment, at Talos Dome (Stenni et al., 2011), Siple Dome (Brook et al., 2005) and Taylor Dome at the divide of Skelton and Mackay Glaciers (Steig et al., 1998); atmospheric temperature increased at 18-20 ka until the Antarctic Cold Reversal (ACR) at 14.5-12.9 ka, after which temperature again rose and then stabilised into the Holocene. These post-glacial increases in temperature are matched by enhanced precipitation in Antarctica (WAIS Divide Project Members, 2013).

Divide Project Members, 2013; Jouzel et al., 2007) and south-west Pacific Ocean sea surface temperature reconstructions (Pahnke and Sachs, 2006), which corresponds with a rapid increase in precipitation (e.g. Morse et al., 1998; WAIS Divide Project Members, 2013) (Figure 2.3B). Atmospheric temperature then temporarily dropped between 14.5 ka and $12.9 \mathrm{ka}$, during the Antarctic Cold Reversal, before plateauing with minor fluctuations through the Holocene (11.6-0 ka) (WAIS Divide Project Members, 2013). Mean- 
while, sea surface temperatures in the Pacific Ocean peaked at $\sim 11$ ka before cooling into the Holocene, which is also seen in reconstructions from the Antarctic continental-shelf (Shevenell et al., 2011) and corresponds to reduced sea ice in Antarctica (WAIS Divide Project Members, 2013). The Ross Sea sector, in the vicinity of Skelton and Mackay Glaciers, experienced minimal changes in atmospheric temperature and precipitation during the Holocene (Steig et al., 1998; Morse et al., 1998).

In summary, the Antarctic climate cooled with pronounced steps during the Mid-Miocene and then the Late Pliocene, into the relatively cold and dry Pleistocene. Ice cores reveal changes in atmospheric temperature over the Late Pleistocene, which show orbitallypaced fluctuations between glacial and interglacial states. It can be inferred that similar variations have occurred at least since the Miocene (Zachos et al., 2001; Lisiecki and Raymo, 2005). Atmospheric warming following the last glacial episode coincides with sea surface warming and increased precipitation until the Holocene, after which the climate remained relatively stable and similar to present.

These changes in Antarctic climate provided the conditions for ice sheet initiation (Zachos et al., 2001; DeConto and Pollard, 2003) and subsequent fluctuations (Huybers, 2006, 2009). In this thesis, outlet glacier behaviour is investigated with respect to past glacial and interglacial climates, of the relatively warm and wet Pliocene and the relatively cool and dry Pleistocene (associated with Research Question 1). The response of glaciers during the the last period of post-glacial warming ( $\sim 20 \mathrm{ka}$ to present) is also explored (associated with Research Question 4).

\subsection{Ice sheet variability in the Ross Sea sector}

Reconstructing the past behaviour of ice sheets and understanding how these variations relate to past climate provides a context for modern and potential future changes in ice sheet volume. The Ross Sea sector of Antarctica, between $\sim 160^{\circ} \mathrm{E}$ and $150^{\circ} \mathrm{W}$, is a region of particular research interest because it is affected by changes in both the East and West Antarctic Ice Sheets; today, this sector includes an area of $\sim 1.65 \times 10^{6} \mathrm{~km}^{2}$ and $0.75 \mathrm{x}$ $10^{6} \mathrm{~km}^{2}$ for the EAIS and WAIS respectively (Rignot et al., 2008). Glacial geological records obtained in this region have improved our understanding of ice sheet variability, identifying the frequency of fluctuations and constraining past ice sheet thickness and extents over the Late Cenozoic (e.g. McKay et al., 2009). Such information, in turn, informs glaciological models that provide a continent-wide perspective of ice sheet variation and dynamics (e.g. Pollard and DeConto, 2009). 


\subsubsection{Miocene, Pliocene \& Pleistocene ice sheet extents}

Following ice sheet initiation on East Antarctic mountain massifs (Bo et al., 2009), grounded ice expanded across the continent to the Ross Sea (Pollard and DeConto, 2009), where it is recorded in sediment cores since 34 Ma ago (e.g. Naish et al., 2001; Barrett et al., 1987). Cyclic variations in glacio-marine sediments, collected in the vicinity of Mackay Glacier, indicate orbitally-paced (40-ka and 125-ka) fluctuations in ice sheet extent into the Miocene (Naish et al., 2001). By the Early Miocene, geophysical surveys suggest an expanded ice sheet had reached the Ross Sea outer continental-shelf (Bartek et al., 1997). Further ice sheet overriding events are recorded in the western Ross Sea (Naish et al., 2001) and Transantarctic Mountains (Sugden and Denton, 2004; Lewis et al., 2007) in the Mid-Miocene. This was a time of significant landscape development (Sugden and Denton, 2004), when many outlet glacier systems became incised (Jamieson et al., 2010).

The last $5 \mathrm{Ma}$ of ice sheet fluctuations are recorded by a comprehensive sedimentary record in the Ross Sea (ANDRILL; Naish et al., 2009), located downstream of Skelton Glacier. Glacio-marine sequences are preserved that represent open water, ice-shelf cover and overriding grounded ice. These are repeated through the core to reveal ice sheet oscillations, which occurred in 41-ka cycles until $\sim 1$ Ma (McKay et al., 2009). Numerical models, forced with stacked benthic marine data that is paced at the same orbital cycles, suggests high magnitude ice sheet fluctuations that possibly led to collapse of the West Antarctic Ice Sheet during the Pliocene (Pollard and DeConto, 2009). During this time, geological evidence indicates that outlet glaciers in the Transantarctic Mountains likely transitioned from subpolar to polar glacial regimes (McKay et al., 2009). A change in basal regime is also shown from flowline modelling of Ferrar Glacier despite only small changes in ice geometry during the Pliocene (Staiger et al., 2006; Johnson and Staiger, 2007; Golledge and Levy, 2011). During the Pleistocene, surface-exposure dating records variations in the geometries of Transantarctic Mountain outlet glaciers, superimposed on long-term ice surface lowering (Joy et al., 2014; Di Nicola et al., 2009, 2012). In the Ross Embayment, at least 7 grounded to floating ice transitions occurred over the last $\sim 800$ ka, suggesting a 100-ka periodicity of ice sheet fluctuations (Naish et al., 2009; McKay et al., 2012). Interglacial conditions during this time were likely similar to today, with an ice-shelf over the ANDRILL-1B drill site, but open water with a less extensive ice-shelf may have existed during Marine Isotope Stage 7 or 5 (McKay et al., 2012).

In summary, detailed geological records and the application of numerical modelling have expanded our understanding of past ice sheet variability since the Miocene. Importantly, grounded ice in the Ross Embayment has been shown to advance and retreat on orbital timescales, as frequently as every $\sim 41$ ka during the Pliocene and Early Pleistocene, and 
with possible ice sheet collapse during warm Pliocene interglacials. Transantarctic Mountain outlet glaciers likely adjusted their dynamics in response to climate, incising their valleys in the process. However, past changes in ice sheet thickness are unconstrained and it is unclear whether outlet glaciers fluctuated together with grounded ice in the Ross Sea over this time. In this thesis, geomorphological mapping, surface-exposure dating and numerical modelling are used to investigate the long-term dynamics and geometries of Skelton and Mackay Glaciers (associated with Research Questions 1, 2 and 3), which have both delivered grounded and floating ice over well-studied geological drill sites.

\subsubsection{Last Glacial Maximum and subsequent deglaciation}

Studying the Last Glacial Maximum (LGM) and subsequent Antarctic deglacial history is important for constraining ice sheet and GIA models, and evaluating ice sheet response to various forcing factors (e.g. sea level, atmospheric and oceanic temperature, and internal feedbacks) (Bentley et al., 2014). In the Ross Embayment, terrestrial and marine evidence record past ice sheet elevations and retreat of both the WAIS and EAIS across the continental-shelf (Anderson et al., 2014).

The maximum extent of grounded ice in the Ross Sea at the LGM has been investigated with seismic and swath bathymetric surveys, and numerous offshore sediment cores. Sampled glacial till and mapped mega-scale glacial lineations reveal that ice previously flowed to the outer continental-shelf, focused through sub-marine troughs alongside raised banks (e.g. Shipp et al., 1999; Greenwood et al., 2012) (Figure 2.4). Here, grounding-zone wedges delimit the LGM ice front, which possibly occurred by $\sim 30.8$ (calibrated radiocarbon, cal.) ka ago in the central Ross Sea (Bart and Cone, 2012) and sometime before $\sim 13$ cal. ka just north of Coulman Island in the western Ross Sea (Shipp et al., 1999; Cunningham et al., 1999). The precise location of the LGM grounding-line remains uncertain, with numerical models simulating more advanced grounded ice close to the continental-shelf edge (Whitehouse et al., 2012; Golledge et al., 2013).

Upstream of the inferred LGM grounding-line, ice sheet thickening is recorded by terrestrial deposits. Glacially-eroded material with far-travelled erratics can be found deposited on islands and peninsulas bordering the Ross Sea, nunataks and valley sides along the Transantarctic Mountains, and in the mouths of deglaciated valleys adjacent to McMurdo Sound. Drift sheets help delimit the thickened LGM ice sheet profile, and have been correlated based on elevation, morphology, weathering and occasional radiocarbon dates between Northern Victoria Land (Terra Nova Drift), Southern Victoria Land (Ross Sea Drift) 


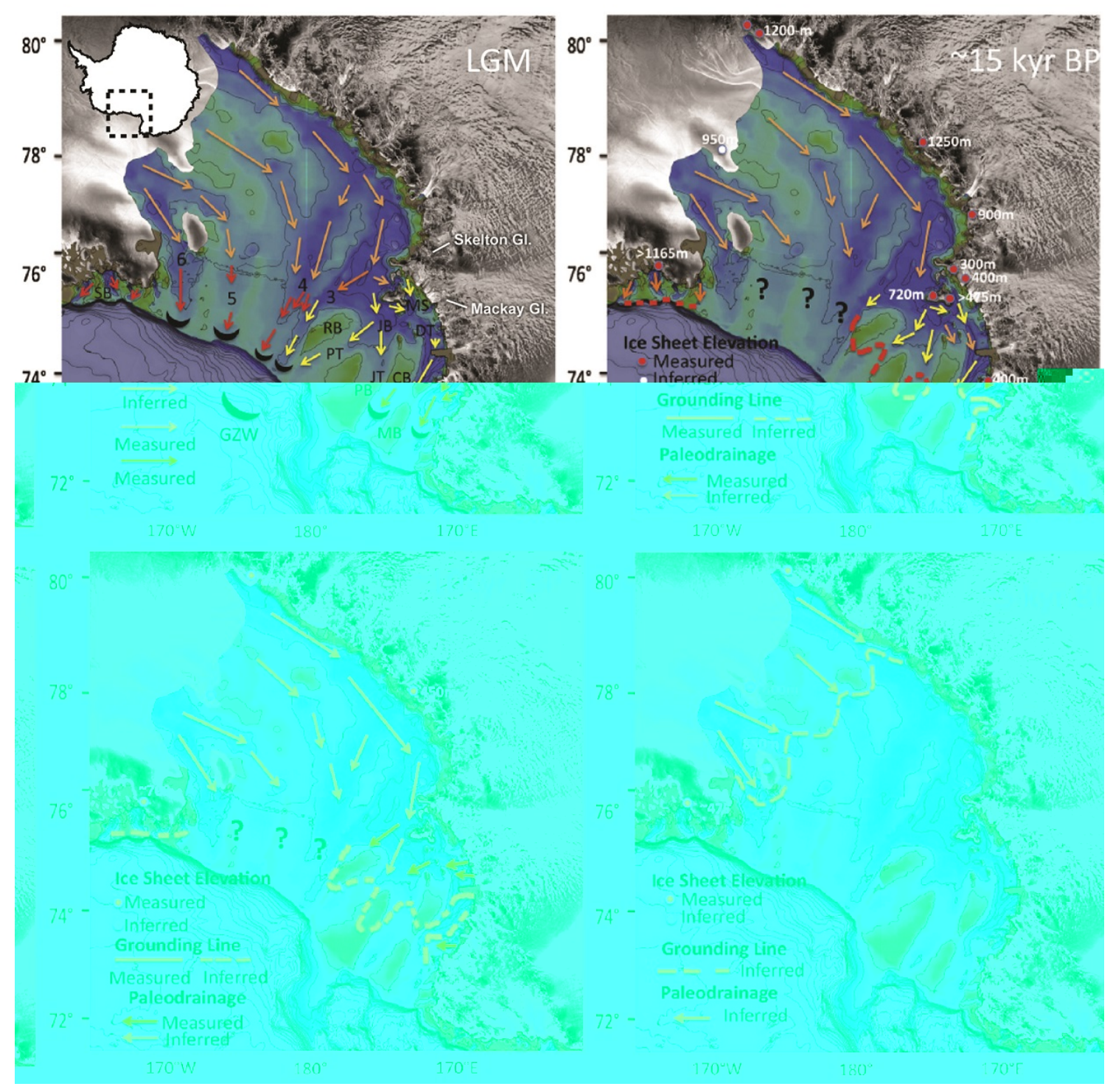

Figure 2.4 Current understanding of LGM to Holocene ice sheet history in the Ross Sea (adapted from Anderson et al., 2014). Measured and inferred ice surface elevations (red and white circles), drainage paths (West Antarctica, orange and red arrows; East Antarctica, orange and yellow arrows), and grounding-lines (solid and dashed red lines) are shown. Bathymetry highlights troughs (dark blue) and banks (green) on the continental shelf. The LGM extent is part-constrained by grounding-zone wedges $(\mathrm{GZW})$, and offshore features are labelled as: MS = McMurdo Sound, DT = Drygalski Trough, CB = Crary Bank, JB = JOIDES Basin (Trough), JT = JOIDES Trough, MB = Mawson Bank, PB = Pennell Bank, PT = Pennell Trough, RB = Ross Bank, SB = Sulzberger Bay, with further troughs numbered. For detailed discussion of this deglaciation evidence see Anderson et al. (2014). The outlets studied in this thesis, Skelton Glacier and Mackay Glacier, are noted in the LGM panel for context.

and the Transantarctic Mountains (Reedy, Scott, Beardmore and Britannia Drifts) (e.g. Stuiver et al., 1981; Orombelli et al., 1990; Bockheim et al., 1989; Denton and Marchant, 2000; Denton and Hughes, 2000; Bromley et al., 2010, 2012). Based on these deposits, LGM ice surface elevations have been recognised as $\sim 1000-1100 \mathrm{~m}$ asl at Scott Glacier (Bromley et al., 2012), $\sim 1100 \mathrm{~m}$ asl later corrected with modelling to $\sim 800-900 \mathrm{~m}$ asl 
at the mouth of the Darwin/Hatherton Glacier system (Bockheim et al., 1989; Anderson et al., 2004), 640 $\mathrm{m}$ asl at Minna Bluff (Denton and Marchant, 2000), $720 \mathrm{~m}$ asl on eastern Ross Island (Denton and Marchant, 2000), 590 m asl on Cape Bird (Dochat et al., 2000), 350-400 $\mathrm{m}$ asl at the mouth of Taylor Valley (Hall and Denton, 2000) and $\sim 300-400 \mathrm{~m}$ asl in Terra Nova Bay (Orombelli et al., 1990). This broadly shows a trend of decreasing surface elevations from the ice sheet interior northwards to the LGM grounding-line (Figure 2.4). However, the inferred LGM ice sheet profile has a very low, and possibly unfeasible surface gradient; numerical models indicate ice was thicker inland with a steeper surface profile at the LGM (e.g. Golledge et al., 2012; Whitehouse et al., 2012). It is therefore possible that these deposits only record a minimum LGM surface elevation, and may instead reflect an ice sheet thermal boundary (Clark, 2011; Golledge et al., 2013) with the ages representing initial deglaciation.

Surface-exposure dating of glacially-deposited erratic clasts can provide a record of thickerthan-present ice geometries that is largely independent from interpretations of depositional landforms. This approach was best illustrated in Marie Byrd Land on the eastern side of the Ross Embayment (Stone et al., 2003). Here, the LGM ice sheet surface was $>\sim 800 \mathrm{~m}$ asl near the coast and $>\sim 1130 \mathrm{~m}$ asl, with reduced thickening, further inland. Surface-exposure dating also determined an LGM surface elevation of $>\sim 1670 \mathrm{~m}$ asl in the Ohio Range near the WAIS divide (Ackert et al., 2007), and $\sim 1100-1400 \mathrm{~m}$ asl and $\sim 1400 \mathrm{~m}$ asl further north at Reedy Glacier (Todd et al., 2010) and upstream Hatherton Glacier (assuming pre-Holocene thickening, Joy et al., 2014), respectively. Again, however, these data only represent exposure after initial deglacial surface lowering and are, therefore, likely minimum estimates of the LGM surface profile. Additional ice sheet surface evidence comes from glaciological techniques, which show the Siple Dome surface, midway along the Siple Coast, was $~ 900-1000$ m asl at the LGM (Waddington et al., 2005). Overall, geological and glaciological data suggest an ice surface profile at the LGM that sloped from mountainous areas in the interior towards the centre of the embayment and the grounding-line on the outer continental-shelf, with interior ice sheet surfaces slightly higher in West Antarctica (by $~ 125$ m; Ackert et al., 2007) and lower or similar to today in East Antarctica (Lilly et al., 2010; Joy et al., 2014; Mackintosh et al., 2014). However, the exact timing and thickness of the LGM ice sheet geometry is uncertain.

Following the LGM, the retreat of grounded ice across the continental-shelf is recorded in sediment cores (e.g. Domack et al., 1999; Licht et al., 1996; Leventer et al., 2006; Mckay et al., 2008); sediment progresses from glacial till to proximal glacimarine to distal glacimarine, with an increasing abundance of diatoms as overriding ice is replaced by open water conditions. The timing of this grounding-line retreat is constrained by radiocarbon ages. These are largely obtained from the acid insoluble organic fraction of 
diatomaceous and other glacimarine sediments as well as occasional carbonate material, and therefore provide minimum dates for ungrounding (Anderson et al., 2014). The calibration of ratiocarbon ages from bulk sediments may be misleading as the extact sources of carbon are uncertain, however despite this, such calibration can provide broad absolute estimates of the minimum age of deglaciation, for comparison to other datasets (e.g. onshore surface-exposure chronologies).

In the western Ross Sea, the grounding-line had retreated south of Coulman Island by $\sim 13$ cal. ka (Cunningham et al., 1999) and was within the Drygalski Trough near the mouth of David Glacier by $\sim 11$ cal. ka (Domack et al., 1999) (Figure 2.4). Retreat was likely faster in these deep troughs with temporary pinning of the grounding-line on shallower parts of the shelf (Figure 2.4); final retreat in Terra Nova Bay, adjacent to the Drygalksi Trough, did not occur until $\sim 8.2 \mathrm{cal}$. ka (Baroni and Hall, 2004). The grounding-line had likely past just north of Ross Island by $\sim 10$ cal. ka (Mckay et al., 2008), again with possible delayed retreat into the shallower coastal areas around McMurdo Sound at $\sim 7.5$ cal. ka (Domack et al., 1999; Licht et al., 1996; Hall et al., 2004). In the central and eastern Ross Sea, the retreat of grounded ice is apparent from recessional moraines and grounding-zone wedges mapped from multibeam swath bathymetry, however the timing of this retreat is unknown (Anderson et al., 2014).

South of Ross Island, retreat of grounded ice is inferred from geological and glaciological data. At upstream Hatherton Glacier, radiocarbon-dated algae in ice-damned lakes indicate the modern glacier geometry had been reached by $\sim 6.8$ cal. ka, which likely corresponds to the timing of grounding-line retreat in the Ross Embayment (Bockheim et al., 1989; Joy et al., 2014). Modelling experiments suggest that this evidence records a lagged response, with grounding-line retreat past the mouth of Hatherton Glacier occurring earlier at 7.1-7.9 ka (Anderson et al., 2004). At Roosevelt Island, in the eastern Ross Sea, a radar-derived ice stratigraphy and time-dependent ice-flow model indicate retreat of the grounding-line past the island before $\sim 3.2 \mathrm{ka}$ (Conway et al., 1999). The modern grounding-line position at Siple Coast was likely reached by $\sim 2$ ka from analysis of flowstripes (Hulbe and Fahnestock, 2007).

Based on these data, two theoretical models of grounding-line retreat have been proposed: 1) 'Swinging gate', whereby retreat occurred from near Coulman Island, along the front of the Transantarctic Mountains, hinged on Roosevelt Island (Conway et al., 1999); and 2) 'Saloon doors', whereby the grounding-line receded first in the central embayment, followed by retreat at the coastal fringes (Ackert, 2008). Numerical ice sheet models constrained by these data favour a mixture of the two styles of deglaciation, with retreat first occurring in the centre of the Ross Sea embayment until roughly Ross Island, and then accelerated grounding-line retreat along the front of the Transantarctic Mountains (e.g. Whitehouse et al., 2012; Golledge et al., 2014b). Further data are required to test these 
deglacial models, especially south of Ross Island where retreat is constrained by a single site.

Ice sheet surface lowering occurred in association with grounding-line retreat, and is recorded around the terrestrial fringes of the embayment with surface-exposure dating. In the eastern Ross Sea sector, surface lowering following the LGM was initially gradual (Stone et al., 2003). This style of thinning continued in the upstream reaches until presentday surfaces were reached at $\sim 2-3 \mathrm{ka}$, however near the coast, an episode of rapid surface lowering of $\sim 400 \mathrm{~m}$ is recorded from $\sim 4 \mathrm{ka}$. In the Transantarctic Mountains, the pattern of ice sheet thinning is less clear. At Reedy Glacier, upstream of the present-day Siple Coast grounding-line, surface-exposure dating of glacial drift deposits suggests surface lowering from $\sim 15 \mathrm{ka}$ into the Holocene, which persisted until $\sim 2-3 \mathrm{ka}$ when presentday glacier surfaces were reached (Todd et al., 2010). Holocene thinning is also recorded further north at Scott and Beardmore Glaciers (Stone et al., pers. comm.), however, like Reedy Glacier, the driving factors responsible are unclear.

In summary, the LGM and subsequent deglaciation is better understood than any previous glacial episode in the Ross Embayment, but large uncertainties still exist regarding the ice sheet configuration and deglacial timing. The extent and thickness of grounded ice at the LGM is partly constrained, however, more records are required, particularly to better delimit maximum ice surface elevations. The timing and style of grounding-line retreat is relatively well understood, more so in the western Ross Sea, but geological constraints south of Ross Island are limited and the response of outlet glaciers to this retreat is uncertain. In this thesis, geomorphological mapping and surface-exposure dating are used to help delimit the LGM ice surface (associated with Research Questions 2, 3 and 4) and record outlet glacier surface lowering during the subsequent deglaciation (associated with Research Question 4). Modelling experiments then explore the drivers responsible for this recorded glacier thinning, with simulations informed by existing offshore evidence.

\subsection{Antarctic contribution to past sea level rise}

The role of Antarctic ice sheets in contributing to future sea level requires an improved understanding of past ice loss (Church et al., 2013). On long timescales, global mean (eustatic) sea level reflects the equilibrium response of ice sheets to climate. On shorter timescales, rates and magnitudes of sea level rise can be deduced from changes in ice sheet geometry. With respect to future sea level, the Miocene, Pliocene and Pleistocene 
interglacials are of interest because they represent the equilibrium response to a presentday or warmer-than-present climate, while the last deglaciation from the LGM holds the potential for understanding the effects of transient sea level rise in a warming climate.

Long-term estimations indicate that eustatic sea level fluctuated together with Antarctic ice sheets on orbital scales (e.g. Miller et al., 2005). During the Miocene, eustatic sea level possibly underwent variations of $\sim 30-60 \mathrm{~m}$, with sea level occasionally lowering to $\sim 20$ $30 \mathrm{~m}$ below present during the Early Miocene (Miller et al., 2005). This inferred increase in ice volume could correspond to ice sheet advances to the outer Ross Sea continentalshelf (Bartek et al., 1997), however it also implies a relatively thin Antarctic ice sheet would have existed at this time. During the Mid-Pliocene, eustatic sea level was $\sim 22 \pm$ $10 \mathrm{~m}$ higher than today (Miller et al., 2012), with large uncertainties derived from GIA modelling of near and far-field sites ( $\sim-30 \mathrm{~m}$; Raymo et al., 2011). Ice sheet modelling simulates $\sim 4 \mathrm{~m}$ of sea level from West Antarctica and $\sim 3 \mathrm{~m}$ from East Antarctica (Pollard and DeConto, 2009), which underestimates the East Antarctic contribution by $\sim 7 \mathrm{~m}$ compared to that which is suggested by sea level reconstructions (Miller et al., 2012). Broadly, a trend of sea level lowering occurred from the Late Pliocene that mirrored global cooling and increasing ice volume (Miller et al., 2005; Lisiecki and Raymo, 2005; Zachos et al., 2001).

Global sea level varied through the Pleistocene with high magnitude fluctuations of atmospheric temperature and Antarctic ice sheet extent (Rohling et al., 2009; Pollard and DeConto, 2009). One Pleistocene interglacial, Marine Isotope Stage 11 ( 400 ka ago), was a period of prolonged atmospheric warmth that contributed $\sim 6-13 \mathrm{~m}$ of sea level relative to present, and suggests collapse of the WAIS with a small contribution from the EAIS (Raymo and Mitrovica, 2012). During the last interglacial, Marine Isotope Stage 5, eustatic sea level was $>6 \mathrm{~m}$ above present (Kopp et al., 2009), with possible contributions from large East Antarctic basins and areas neighbouring the WAIS in addition to ice loss from West Antarctica (Bradley et al., 2013; Pingree et al., 2011). Sea level likely rose in metre-scale jumps during this time (Blanchon et al., 2009), suggesting dynamic, nonlinear ice loss from Antarctica.

The LGM and subsequent deglaciation is recorded in numerous near-coastal sea level archives. A 14-ka window (at 30-16 ka BP) represents the LGM when eustatic sea level was $<120$ m below present (Stanford et al., 2011; Lambeck et al., 2014). Sea level rose from a possible LGM minimum of $134 \mathrm{~m}$ below present (at $21 \mathrm{ka} \mathrm{BP}$ ) until $\sim 8.3 \mathrm{ka}$ BP (Lambeck et al., 2014), after which the rate of sea level rise progressively decreased. The rise in sea level is characterised by several meltwater pulses, known as MWP 19ka, MWP-1A and MWP-1B. At $\sim 19.5-18$ ka BP, eustatic sea level rose by $\sim 10-15 \mathrm{~m}$ (Yokoyama et al., 2000; Lambeck et al., 2014), reflecting the onset of substantial deglacia- 
tion. The largest recorded meltwater pulse is MWP-1A, identified in Barbados (Fairbanks, 1989), Sunda (Hanebuth et al., 2000) and Tahiti (Bard et al., 1996; Deschamps et al., 2012) from $\sim 14.6 \mathrm{ka} \mathrm{BP}$. Once initiated, sea level rose by $\sim 20 \mathrm{~m}$ over $\sim 300$ years at a rate of $>40 \mathrm{~mm} \mathrm{a}^{-1}$ (Deschamps et al., 2012; Lambeck et al., 2014). Meltwater pulse 1B has thus far only been found in Barbados (Bard et al., 2010), with statistical analysis of combined sea level records questioning the occurrence of a rapid rise at this time (Fleming et al., 1998; Stanford et al., 2011; Lambeck et al., 2014).

The sources of these meltwater pulses are still not fully resolved (Clark et al., 2009; Carlson and Clark, 2012). Meltwater pulse 19-ka has been primarily attributed to the retreat of Northern Hemisphere ice sheets (Clark et al., 2004), although at least a small contribution came from Antarctica (Weber et al., 2011). Despite only an isolated appearance in far-field sea level records, Antarctic ice loss likely contributed to sea level rise around the time of MWP-1B (Mackintosh et al., 2011; Weber et al., 2014; Golledge et al., 2014b; Stanford et al., 2011; Lambeck et al., 2014). The sources of MWP-1A are less certain. In the Northern Hemisphere, melting of the Laurentide, Cordilleran and European ice sheets contributed $\sim 6-8 \mathrm{~m},<1 \mathrm{~m}$ and $\sim 1-3 \mathrm{~m}$ ice equivalent sea level over this period, respectively (e.g. Peltier, 2005; Tarasov and Peltier, 2005, 2006; Tarasov et al., 2012; Gregoire et al., 2012). It has been suggested that $\sim 4-7 \mathrm{~m}$ of this Northern Hemisphere contribution occurred during MWP-1A (Carlson and Clark, 2012). Assuming a rise in sea level between $12 \mathrm{~m}$ and $22 \mathrm{~m}$ during MWP-1A (Deschamps et al., 2012), this leaves $\sim 5-18 \mathrm{~m}$ of sea level potentially needing to be sourced from the Southern Hemisphere.

The WAIS and EAIS have been suggested as alternative sources based upon a modelled melting scenario that matches far-field sea level indicators (Clark et al., 2002). A recent record of iceberg-rafted debris identifies several episodes of ice discharge in Antarctica, the largest of which corresponds with MWP-1A (Weber et al., 2014), although it is not possible to estimate the magnitude of ice loss from this record. Ice sheet models can help investigate the magnitude and cause of sea level contributions. Forced with coupled ocean-atmosphere climate model, a similar pattern of ice loss is simulated in Antarctica as found in the debris record (Figure 2.5), supporting Antarctic ice sheets as a contributor to MWP-1A (Golledge et al., 2014b). However, significant deglaciation at this time is not recorded in the available geological data (e.g. Stone et al., 2003; Bentley et al., 2006; Mackintosh et al., 2007). Importantly, for Antarctic ice sheets to be the primary source of MWP-1A, they must have contained a total of at least $7 \times 10^{6} \mathrm{~km}^{3}$ more ice at the LGM than at present. This is roughly 1.2-2 times that determined from ice sheet models constrained by current geological data (e.g. Mackintosh et al., 2011; Whitehouse et al., 2012; Golledge et al., 2014b).

In summary, past changes in eustatic sea level are recorded sporadically through the Late Cenozoic. Miocene and Pliocene sea level fluctuated largely with Antarctic ice sheets on 


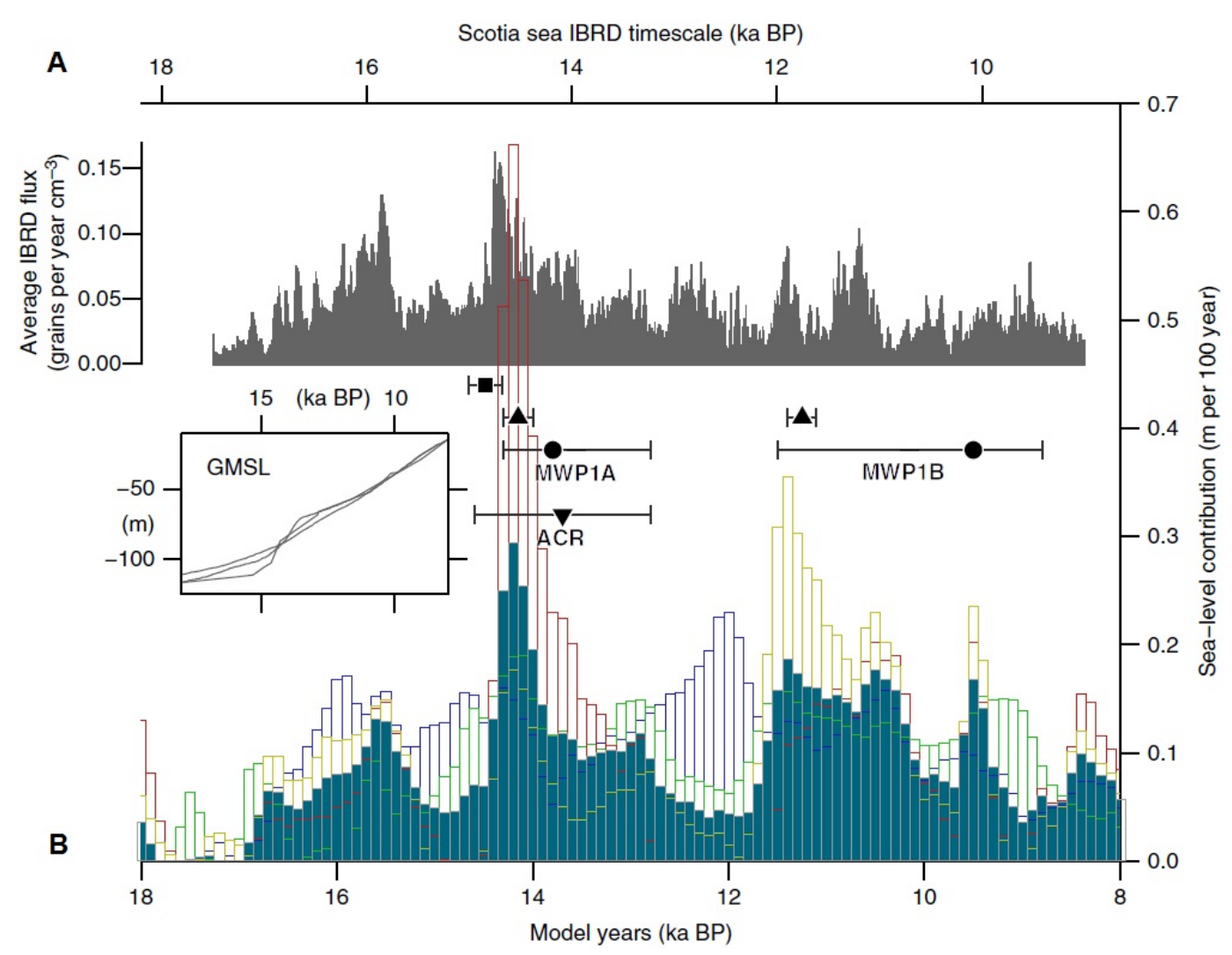

Figure 2.5 Sea level contributions from Antarctic ice sheets following the LGM (after Golledge et al., 2014b). A) Iceberg-rafted debris (IBRD) variability in the Scotia Sea (Weber et al., 2014), revealing a relatively large ice discharge event around the time of MWP-1A. B) Simulated sea level contribution from Antarctica for a range of ocean temperature forcings (coloured bins) and ensemble mean (solid blue bins), showing a similar pattern of Antarctic ice loss to that in the IBRD record (Golledge et al., 2014b).

The timings of MWP-1A and MWP-1B are shown with a square (Deschamps et al., 2012), triangles (Liu and Milliman, 2004) and circles (Stanford et al., 2011). The inset shows global mean sea level (GMSL) from various records over this time (Deschamps et al., 2012; Stanford et al., 2011; Clark et al., 2009).

orbital timescales, supporting the idea that large rises in sea level occurred from the collapse of the WAIS and parts of the EAIS. Episodes of sea level rise during the Pleistocene were predominantly sourced from both Northern Hemisphere and Antarctic ice sheets, and sea level records indicate significant, rapid meltwater events. However, estimates of past sea level, whether of highstands/lowstands or rapid sea level rise, rely on far-field reconstructions that are inadequately supported by Antarctic evidence. Therefore, any new data from Antarctica helps improve our understanding of Antarctica's contribution to past sea level change. This thesis investigates long-term ice sheet fluctuations and the potential contribution from grounded ice in the western Ross Sea to meltwater pulses following the LGM (associated with Research Questions 2, 3 and 4). 


\subsection{Constraining past ice sheet behaviour}

In this research, past ice sheet geometries and dynamics will be constrained at two outlet glaciers that feed into the western Ross Sea, the importance of which has been demonstrated with respect to understanding potential ice sheet behaviour and sea level response.

As discussed, the Miocene, Pliocene and Pleistocene ice sheet extents have been broadly determined offshore with stratigraphic profiles of grounding-zone wedges on the continental shelf (e.g. Bartek et al., 1997) and sediment cores recording the fluctuations of grounded ice (e.g. Naish et al., 2009). To gauge the upstream behaviour, terrestrial evidence is required. In mountainous regions of Antarctica, advancing ice sheets can produce a geological signature, which can be identified as erosional surfaces (e.g. Sugden and Denton, 2004) and moraines (e.g. Lewis et al., 2007). Fluctuations in ice sheet extent typically occur together with variations in ice surface elevation. Surface-exposure dating at inland nunataks can record long-term changes in ice surface lowering (e.g. Suganuma et al., 2014), and also allows for the investigation of a fluctuating surface elevation over time (e.g. Lilly et al., 2010). It is for these reasons that surface-exposure dating is used here, together with the assessment of geomorphological signatures.

The best preserved records of past ice sheet geometry and behaviour come from the LGM and subsequent deglaciation. Offshore sediment cores and onshore deposits record the horizontal and vertical extent of the ice sheet, and are largely temporally-constrained with radiocarbon dating of organic material. The coherence of these chronologies, however, is limited by sediment that is unsuitable for dating (e.g. a lack of carbonate material and input of 'old' carbon from the bioturbation of sediment) and uncertain marine reservoir effects (with corrections in the Ross Sea ranging from 1.1 ka to >1.4 ka) (Hall et al., 2010; Anderson et al., 2014). Furthermore, terrestrial organic material is sparse in Antarctica and what does exist sources most of its carbon from the marine environment. Surfaceexposure dating is an alternative approach for determining changes in ice sheet surface elevation, highly suited to terrestrial dating in Antarctica (Chapter 3; Balco, 2011). Here, surface-exposure dating is used to determine the LGM surface elevation and subsequent surface lowering, and by targeting multiple nunataks this technique identifies spatial differences in the timing and magnitude of ice surface changes.

Ice sheet models, constrained by geology, can be used to recognise the Antarctic-wide pattern and drivers of recorded ice sheet behaviour (e.g. Pollard and DeConto, 2009; Mackintosh et al., 2011; Whitehouse et al., 2012; Golledge et al., 2012; Briggs et al., 2013; Golledge et al., 2014b). However, as discussed, large misfits exist between recorded and simulated changes, which preclude adequate evaluation of outlet glacier dynamics. Glacier flowline models are better suited to understanding the behaviour of outlet glaciers from geological data constraints. Such modelling can be used to investigate time- 
dependent steady-state flow regimes (e.g. Johnson and Staiger, 2007; Golledge and Levy, 2011) and the controls on ice dynamics through transient simulations (e.g. Jamieson et al., 2012), which are not attainable with more complex and computationally-expensive models (e.g. Gagliardini et al., 2013). Here, numerical flowline modelling is used in combination with surface-exposure dating chronologies at Skelton and Mackay Glaciers.

This thesis provides new geological data that constrains long-term ice sheet fluctuations in the vicinity of the Transantarctic Mountains and western Ross Sea, as well as the LGM configuration and subsequent thinning response of outlet glaciers in this region. Flowline modelling helps to decipher the time-dependent flow of these these glaciers and the transient dynamics during deglaciation.

\subsection{Research questions}

The response of ice sheets to climate change and the extent to which such a response is affected by internal mechanisms remains unclear. This imperfect understanding motivated the following research questions, which are focused on reconstructing the past configurations and ice dynamics of two Transantarctic Mountain outlet glaciers. Investigation of Skelton Glacier and Mackay Glacier was directed by questions $1 \& 2$ and $3 \&$ 4 , respectively.

1. What are the main environmental variables influencing the flow of Skelton Glacier during present and past climates?

2. To what extent has Skelton Glacier undergone a stable or fluctuating geometry during the Late Cenozoic?

3. How does the flow regime of Mackay Glacier vary temporally and spatially?

4. What was the style and mechanism of thinning at Mackay Glacier during the last deglaciation?

To answer these questions, surface-exposure dating and numerical glacier modelling were utilised. These methods will be outlined in the next chapter. 


\section{Chapter 3}

\section{Methodology}

The research undertaken in this thesis is focused on better understanding past glacier configurations and ice dynamics. This is carried out at two Transantarctic Mountain outlet glaciers with the use of two principal techniques. Terrestrial cosmogenic nuclide surfaceexposure dating is conducted in order to determine past ice sheet surface elevations and glacier thinning through time. Meanwhile, glaciological modelling is applied to investigate glacier dynamics under past and present conditions. In this chapter, I describe cosmogenic nuclides and the application of surface-exposure dating with respect to Antarctica. I also provide a description of the glaciological modelling approach used here.

\subsection{In situ-produced cosmogenic nuclides}

\subsubsection{Cosmic-ray flux at the Earth's surface}

The technique of surface exposure dating exploits cosmic radiation that reaches the Earth's atmosphere and the subsequent production of cosmogenic nuclides at or just below the surface. This process can be divided into three main stages: the production of 1) primary cosmic rays, 2) secondary cosmic rays, and 3) cosmogenic nuclide reactions, which will now be discussed in turn.

Cosmic radiation can be composed of either low energy $(<0.1 \mathrm{GeV})$ solar-derived particles (Masarik and Reedy, 1995) or high energy galactic particles (0.1-10 GeV) (Lal and Peters, 1967). Radiation of galactic origin is more important for nuclide production and consists of protons (87\%), alpha particles (12\%) and other heavy nuclei ( $\sim 1 \%)$ (Masarik and Beer, 1999). The intensity of these primary, incoming rays is heavily influenced by the Earth's predominantly dipole geomagnetic field. Rays must exceed a minimum momentum (or rigidity) in order to penetrate the magnetic field, which is dependent on the angle of incidence of the rays and their location relative to magnetic field lines (Figure 3.1; Dunai, 2010). At the poles, for example over Antarctica, rigidity is low and therefore more primary particles can penetrate; at the equator the opposite is true, with lower energy particles unable to penetrate.

Having reached the Earth's atmosphere, incoming rays react to form secondary cosmic rays (Figure 3.2). A cascade of these secondary particles occurs as the primary rays in- 


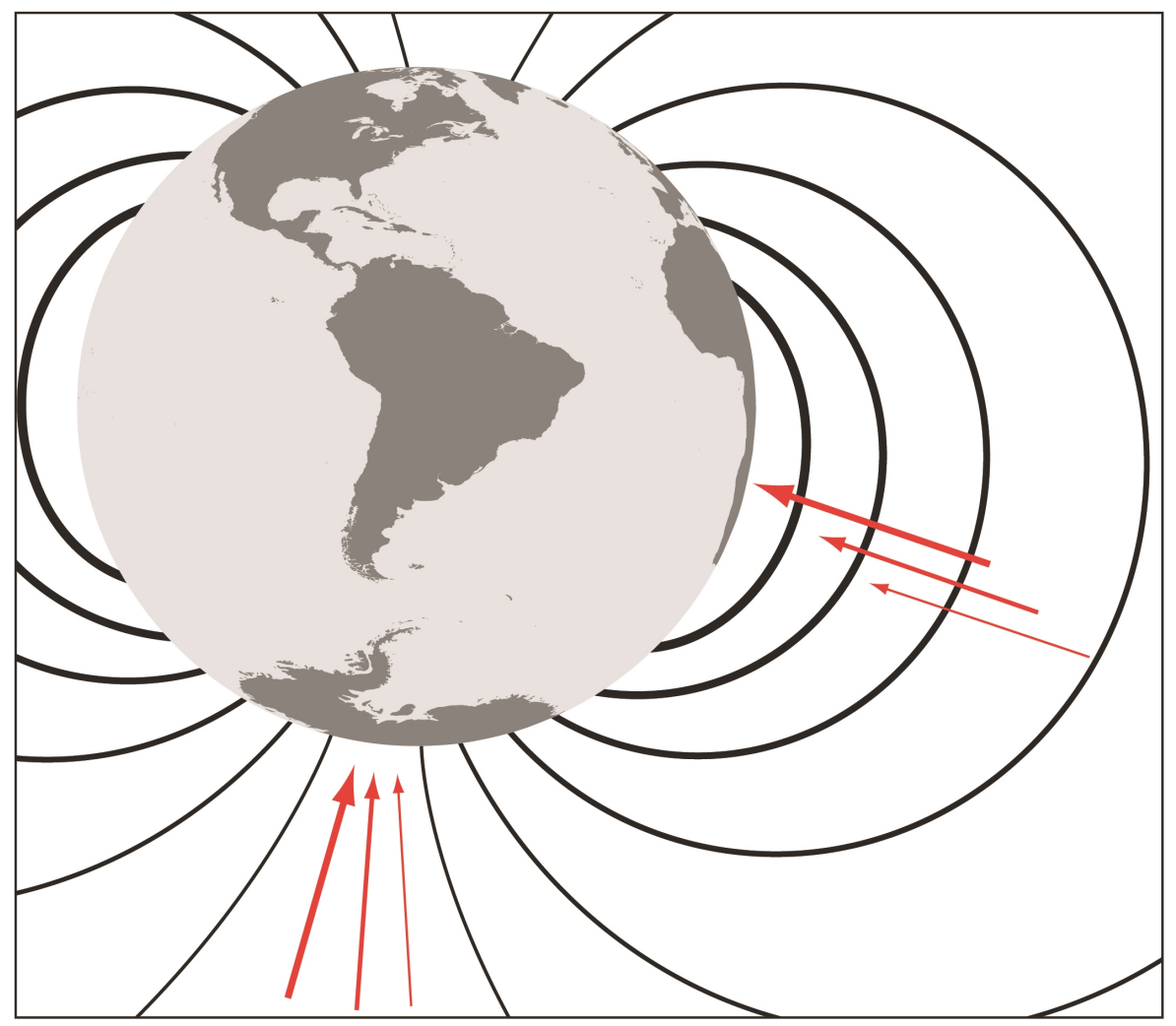

Figure 3.1 Geomagnetic field and affect on incoming primary cosmic rays, shown schematically (adapted from Darvill, 2013). All cosmic rays must exceed the cut-off rigidity. In Antarctica, converging magnetic field lines provide a low cut-off rigidity, allowing most primary rays to reach the atmosphere and resulting in higher cosmogenic nuclide production rates. Whereas only high rigidity primary rays $(>10 \mathrm{GeV})$ enter the atmosphere at the equator.

teract with gas nuclei in the atmosphere, forming both high energy nucleons (protons and neutrons) and mesons (e.g. muons, kaons and pions) (Gosse and Phillips, 2001). Energy becomes scattered or absorbed as the production of secondary rays cascades down through the atmosphere, leading to a near-exponential decrease in ray intensity towards the Earth's surface. The length of this attenuation is dependent on the energy of incoming rays, which is $\sim 130 \mathrm{~g} \mathrm{~cm}^{2}$ at high latitudes, like Antarctica, and $\sim 150 \mathrm{~g} \mathrm{~cm}^{2}$ near the equator (Dunai, 2010). It is during these reactions that meteoric cosmogenic nuclides are produced.

Finally, in situ cosmogenic nuclides are produced as these secondary rays interact with nuclei at the Earth's surface and shallow sub-surface. Nuclides form in a range of lithologies (inclusive of igneous, metamorphic and sedimentary rocks) with the specific nuclide (e.g. ${ }^{3} \mathrm{He},{ }^{10} \mathrm{Be},{ }^{14} \mathrm{C},{ }^{21} \mathrm{Ne},{ }^{26} \mathrm{Al},{ }^{36} \mathrm{Cl}$ ) dependent on rock composition and hence, the target minerals available. Reactions are typically either nucleogenic or muogenic with the former producing most nuclides, involving fast and high energy neutrons (Table 3.1). 


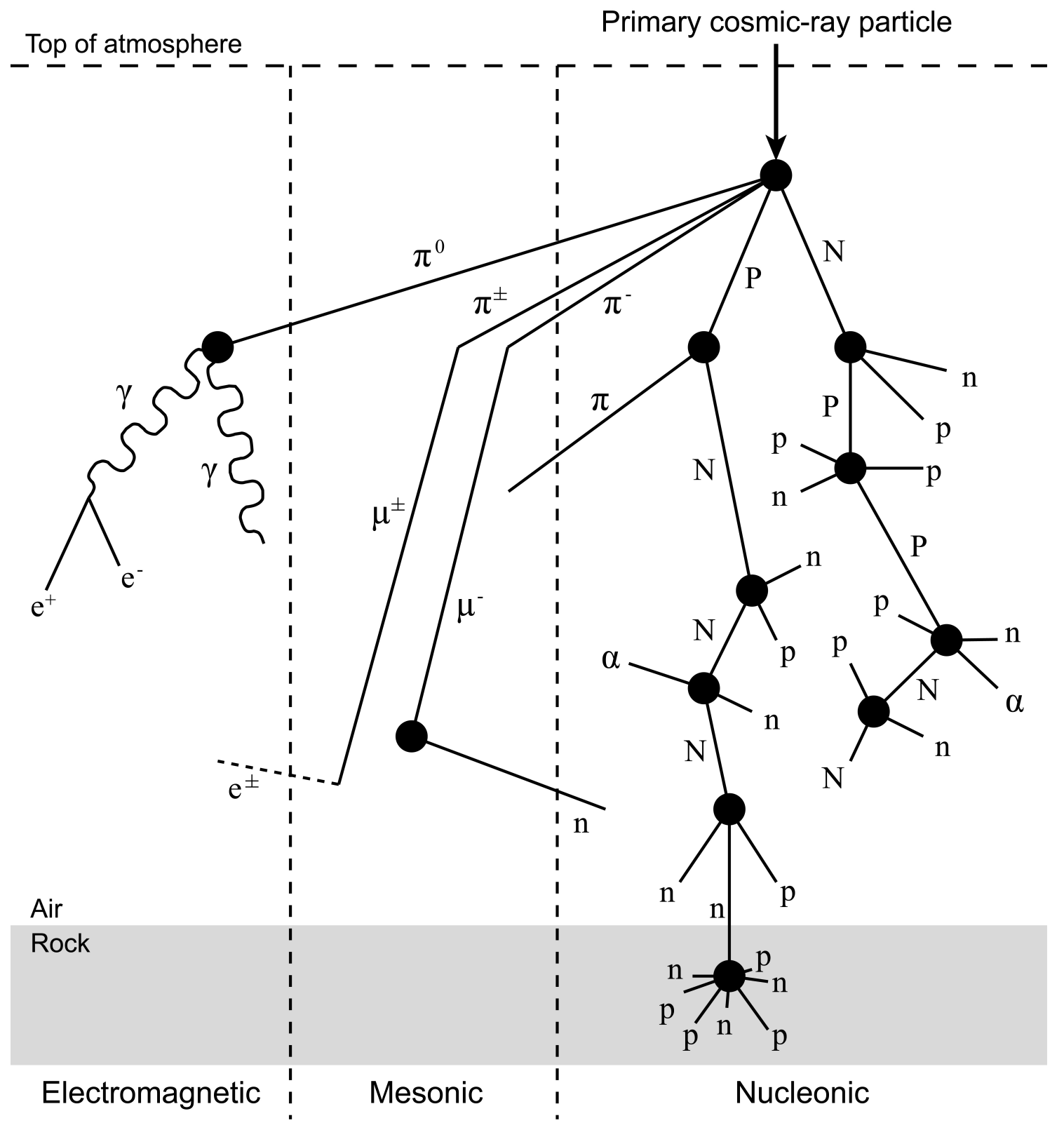

Figure 3.2 Cascade of secondary cosmic rays that are produced in the atmosphere and rock (adapted from Gosse and Phillips, 2001; Dunai, 2010). In situ cosmogenic nuclide interaction in rock is shown for ${ }^{10} \mathrm{Be}$. Abbreviations: $\mathrm{n}(\mathrm{N})$, neutron (carrying nuclear cascade); $\mathrm{p}(\mathrm{P})$, proton (carrying nuclear cascade); $\alpha$, alpha particle; $\mathrm{e}^{ \pm}$, electron or positron; $\gamma$, gamma-ray photon; $\pi$, pion; $\mu$, muon. 
Table 3.1 Cosmogenic nuclides. Common nuclides and their reactions at the Earth's surface.

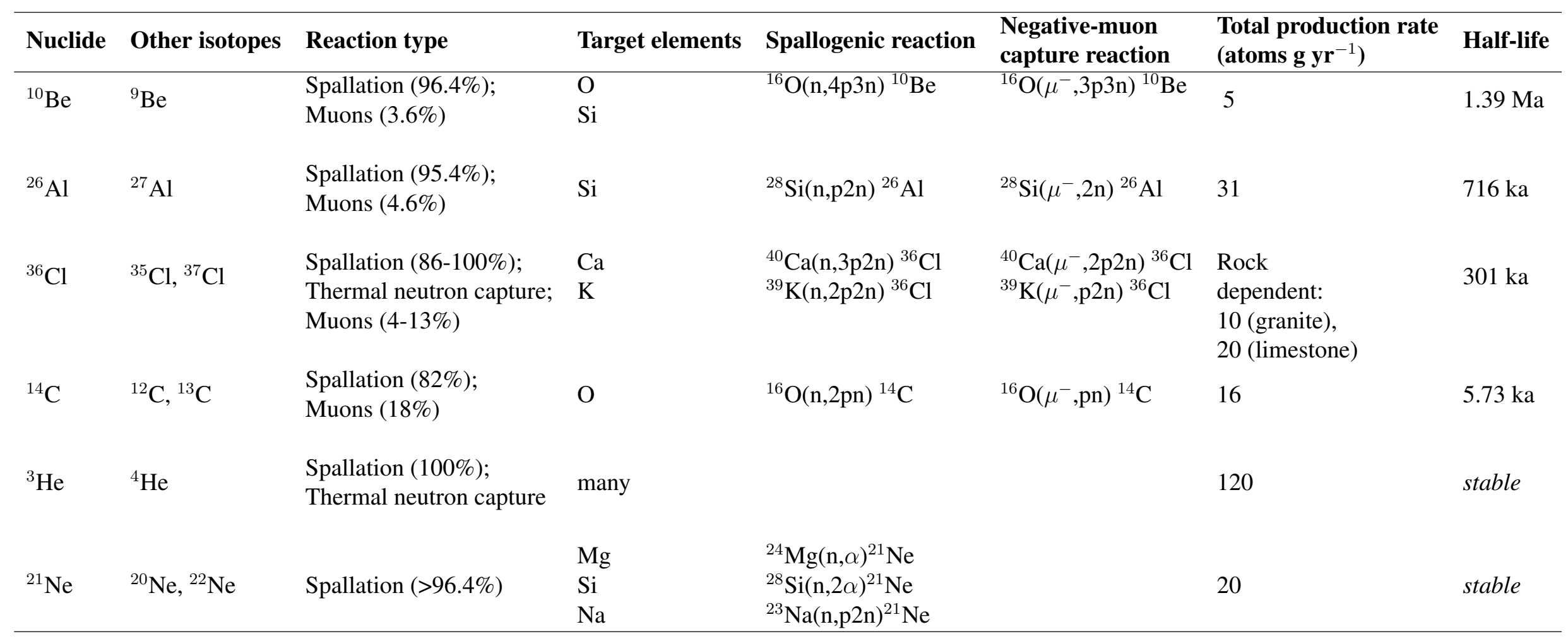

Modified after Gosse and Phillips (2001), Ivy-Ochs and Kober (2008), Dunai (2010). 
The flux of neutrons attenuates down through the rock surface as reactions occur with target elements. This attenuation is exponential with depth, reducing the neutron flux to $<1 \%$ below $\sim 3 \mathrm{~m}$, with the depth dependent on rock density (Lal, 1991). The lower mass of muons relative to neutrons makes them less reactive, which allows them to penetrate deeper into rock. As a result, muon reactions become dominant below $\sim 2 \mathrm{~m}$ and are nearexclusive below $\sim 3 \mathrm{~m}$ in rock (or $1000 \mathrm{~g} \mathrm{~cm}^{2}$ ). Following nuclide production, radioactive isotopes experience a time-dependent decay. Nuclides continue to accumulate in a rock surface until, if the nuclide is radioactive, production equals decay. At this point, secular equilibrium (saturation) is reached, which is typically after about three to four respective half-lives (Figure 3.3).
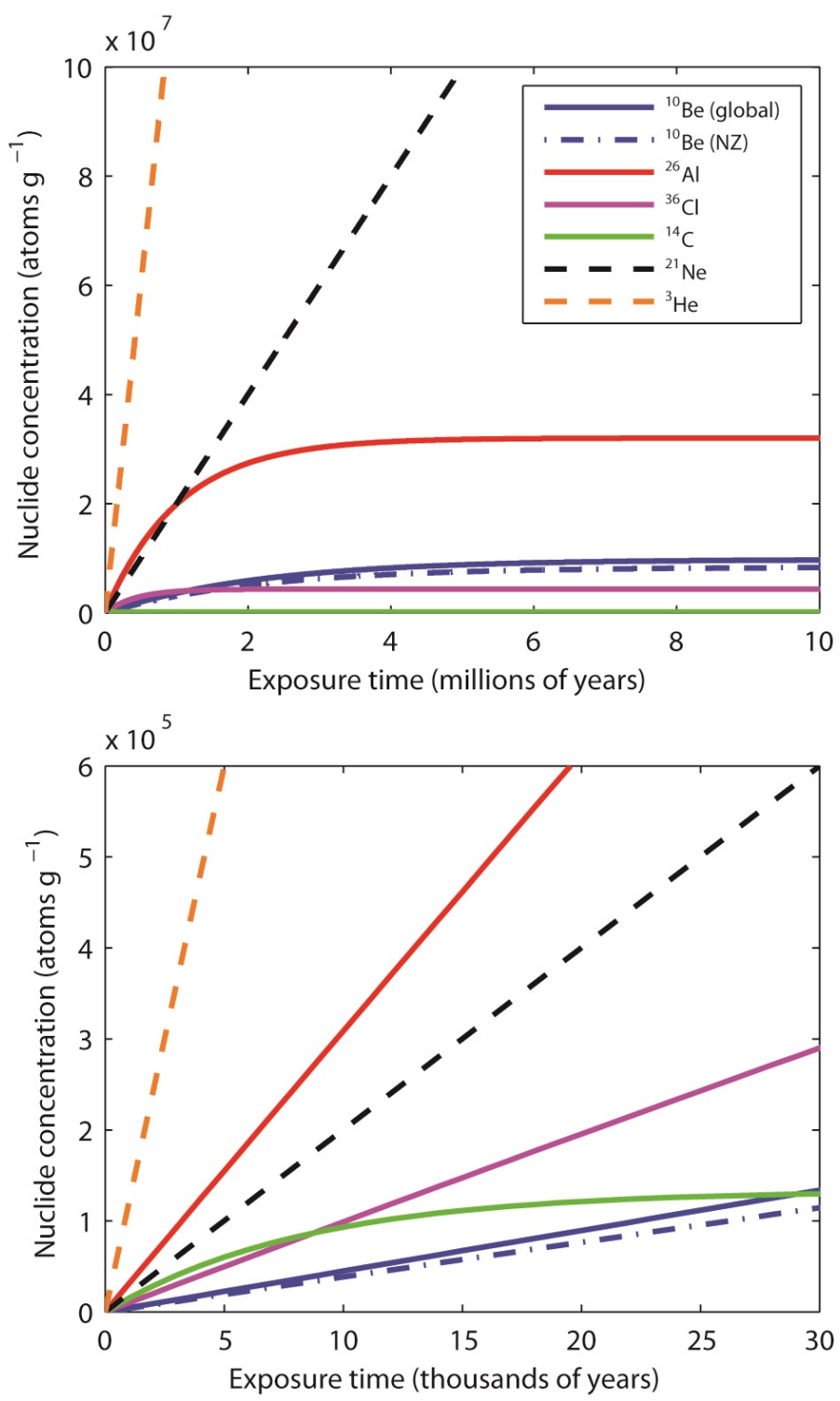

Figure 3.3 Cosmogenic nuclide concentrations through time (SLHL). Concentrations are shown for both stable (dashed line) and radioactive (solid line) isotopes, with ${ }^{10} \mathrm{Be}$ calculated using both global and New Zealand (NZ) production rates. Radioactive isotopes become saturated over time, preventing continuous increases in concentration. 


\subsubsection{Nuclide production in quartz}

In this thesis, I focus on quartz-bearing lithologies that accumulate ${ }^{10} \mathrm{Be}$ and ${ }^{26} \mathrm{Al}$. These nuclides are ubiquitous and relatively well understood.

The concentration of a nuclide (atoms $\mathrm{g}^{-1}$ ) in a mineral (e.g. quartz) through time $\left(N_{(t)}\right)$ can be calculated with the following equation:

$$
N_{(t)}=\frac{P_{(0)}}{\lambda+\frac{\rho \varepsilon}{\Lambda}}\left(1-e^{-\left(\lambda+\frac{\rho \varepsilon}{\Lambda}\right) t_{e x p}}\right)+N_{i n h} e^{-\lambda t_{e x p}}
$$

where $P_{(0)}$ is the site-specific production rate (atoms $\mathrm{g}^{-1} \mathrm{a}^{-1}$ ), $\lambda$ is the decay constant $\left(\mathrm{a}^{-1}\right), \rho$ is the rock density $\left(\mathrm{g} \mathrm{cm}^{3}\right), \varepsilon$ is the (subaerial) erosion rate $\left(\mathrm{cm} \mathrm{a}^{-1}\right), \Lambda$ is the attenuation length $\left(\mathrm{g} \mathrm{cm}^{2}\right), t_{\text {exp }}$ is the exposure age of the surface (years), and $N_{\text {inh }}$ is the pre-existing nuclide concentration, referred to as 'inheritance'. Commonly, calculated concentrations are then normalised to the sea-level high-latitude $\left(<60^{\circ}\right)$ production rate (SLHL) for comparison between sites.

Assuming zero inheritance or erosion, Eq. 3.1 can be simplified to:

$$
N_{(t)}=\frac{P_{(0)}}{\lambda}\left(1-e^{-\lambda t_{e x p}}\right)
$$

In numerous situations, an exposed surface can be become buried (for example, by glacial ice, accumulating sediment or rockfall deposits), at which point nuclides are no longer produced. During an episode of (infinitely deep) burial $\left(t_{\text {bur }}\right)$, a nuclide concentration evolves, partly dependent on the subglacial erosion component $(\varepsilon)$, as follows:

$$
N_{(t)}=N_{i n h} e^{-1\left(\lambda+\frac{\rho \varepsilon}{\Lambda}\right) t_{b u r}}
$$

During both exposure and burial, nuclide concentrations are produced and decay at different rates dependent on the cosmic ray flux, attenuation length and rock density. For a range of input values, the greatest variance of resulting nuclide concentrations occurs near saturation during exposure, while decay is faster for denser rock and shorter attenuation lengths when buried (Figure 3.4).

As previously mentioned, the nuclides of ${ }^{10} \mathrm{Be}$ and ${ }^{26} \mathrm{Al}$ have different half-lives (Table 3.1). This relationship can be exploited to reveal the evolution of nuclide concentrations through time, which in turn, can inform us about surface processes. For example, for a 
continuously exposed surface, the concentration of ${ }^{10} \mathrm{Be}$ increases until it reaches secular equilibrium while the ratio of ${ }^{26} \mathrm{Al}$ to ${ }^{10} \mathrm{Be}$ slightly decreases as the lower half-life of ${ }^{26} \mathrm{Al}$ causes it to reach secular equilibrium sooner (Figure 3.5). Surfaces can experience different concentration trajectories despite continuous exposure as a result of subaerialweathering; a steady-state erosion island represents the area within which a continuously exposed surface can exist (Figure 3.6). Following exposure, when a surface becomes buried and hence, protected from cosmic rays, the concentration of ${ }^{26} \mathrm{Al}$ decays more quickly than that of ${ }^{10} \mathrm{Be}$; the ${ }^{26} \mathrm{Al} /{ }^{10} \mathrm{Be}$ ratio decreases across burial isochrons, in line with radioactive decay (Figure 3.7).
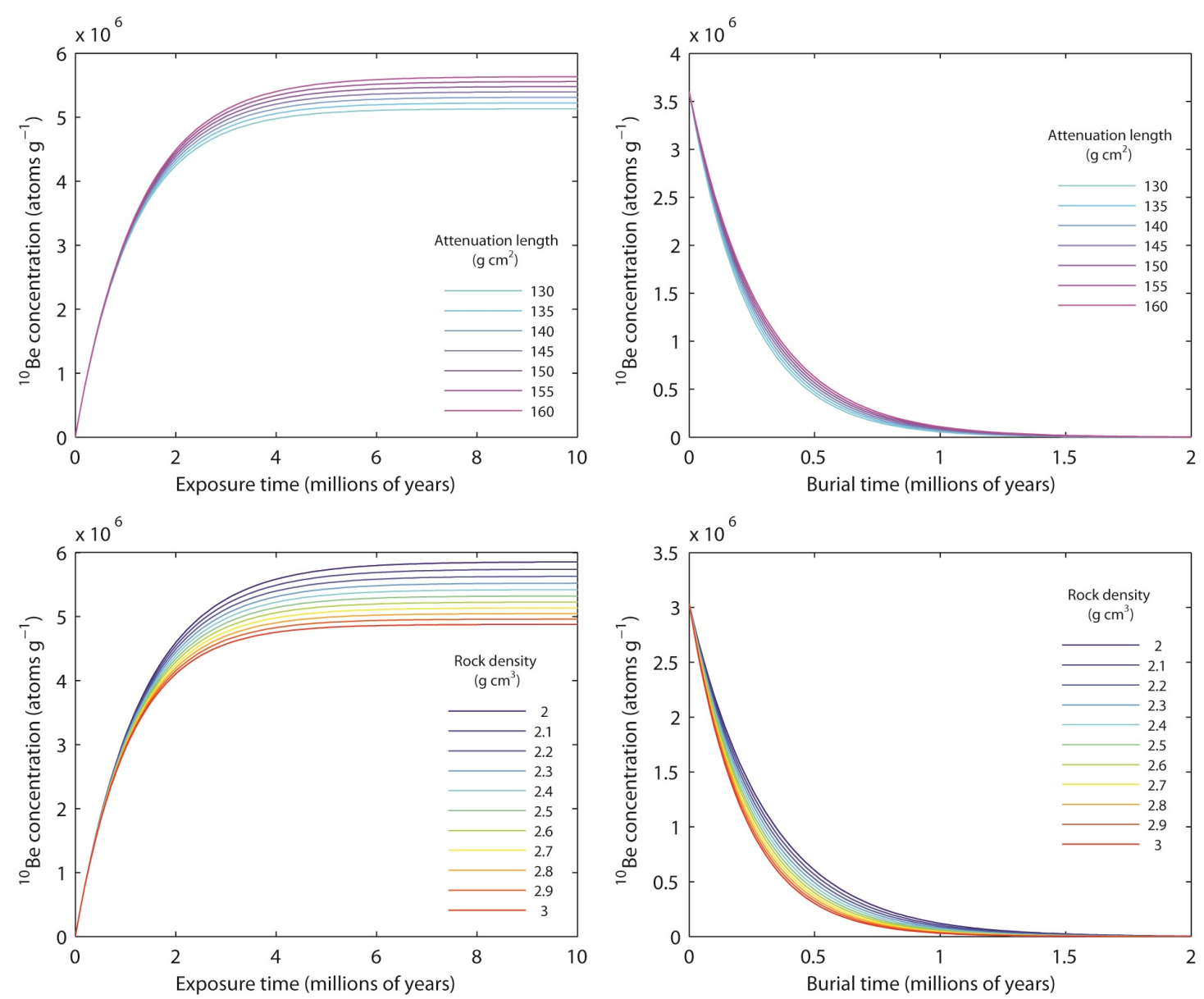

Figure 3.4 Evolution of nuclide concentrations from different attenuation lengths and rock densities during continuous periods of exposure and burial. During exposure (left panels), the greatest variance between attenuation length and rock density values occurs near saturation. During burial (right panels), concentrations diverge until $\sim 500 \mathrm{ka}$, with decay of ${ }^{10} \mathrm{Be}$ faster for denser rock and shorter attenuation lengths. Nuclide concentrations calculate in these examples assume complete burial by overriding ice with subglacial erosion of $18 \mathrm{~mm} \mathrm{ka}^{-1}$, and subaerial weathering of $0.2 \mathrm{~mm} \mathrm{ka}^{-1}$ during exposure. 


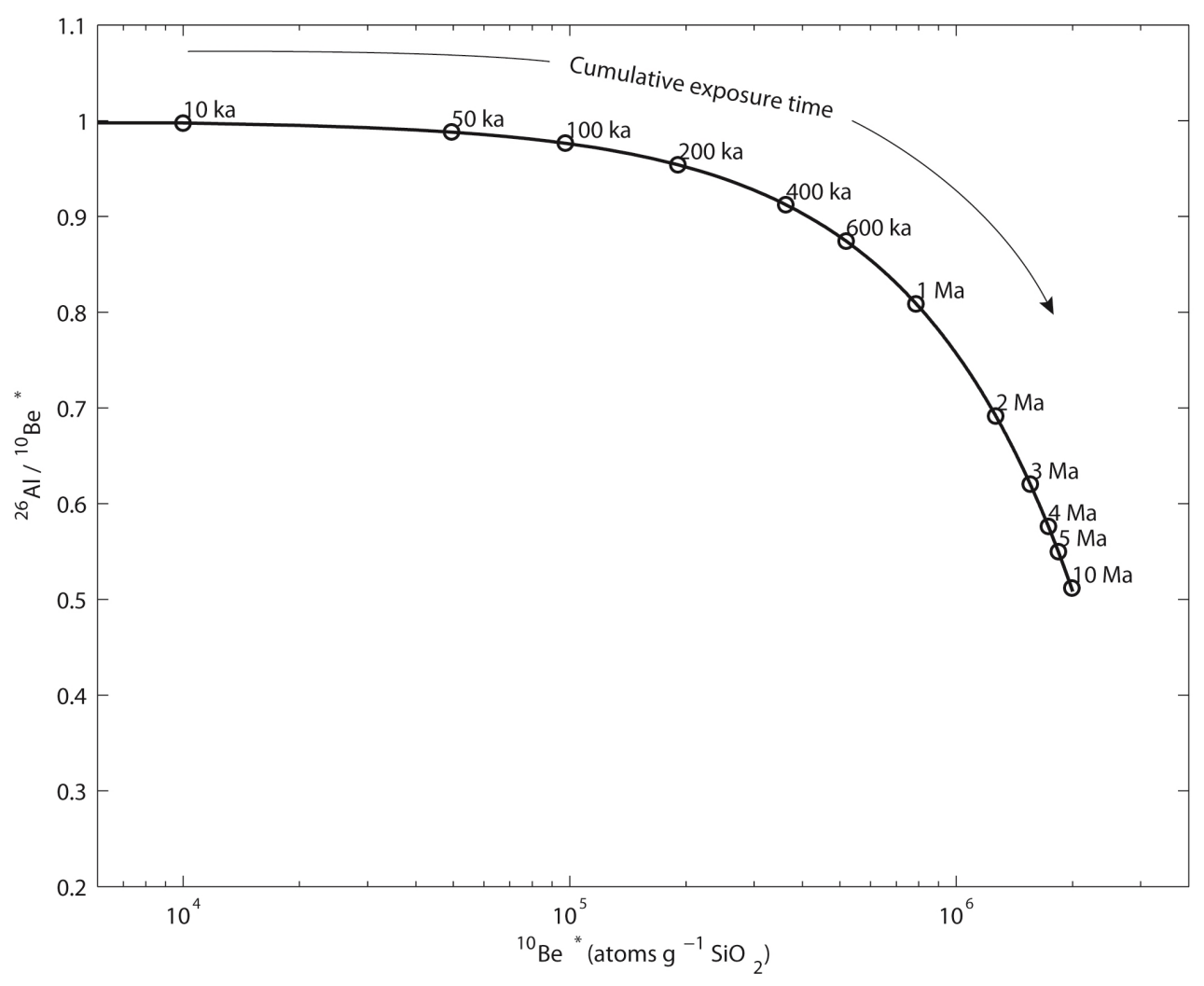

Figure 3.5 Nuclide concentrations over time with continuous exposure. The ${ }^{10} \mathrm{Be}$ concentration increases until it becomes saturated and is at secular equilibrium, after about 3-4 half-lives. As the half-life is less for ${ }^{26} \mathrm{Al}$, the ${ }^{26} \mathrm{Al} /{ }^{10} \mathrm{Be}$ ratio starts to decrease before ${ }^{10} \mathrm{Be}$ reaches secular equilibrium. Concentrations have been normalised to SLHL.

\subsubsection{Temporal and spatial variations in production rates}

The production rate of a nuclide is integral to calculating a surface's concentration with respect to time (Eq. 3.1 and 3.2). Absolute determination of a production rate typically requires the measurement of a nuclide concentration in a surface of known (independently dated) exposure age, along with scaling schemes to extrapolate these local, time-averaged production rates to present-day sea level and high latitude. Such scaling accounts for both spatial and temporal variations in production rate, largely associated with geomagnetic field effects; as previously explained, cosmic ray intensity is greater at altitude than sea level and at the pole than the equator.

Using published calibration site production rates and an averaging procedure for different scaling schemes, global reference production rates have been determined. Until recently, these were assumed to be $5.1 \pm 0.3$ atoms $\mathrm{g} \mathrm{a}^{-1}$ and $31.1 \pm 1.9$ atoms $\mathrm{g} \mathrm{a}^{-1}$ for ${ }^{10} \mathrm{Be}$ and ${ }^{26} \mathrm{Al}$ respectively, at $1013.25 \mathrm{hPa}$ at high latitude (Stone, 2000), or $4.96 \pm 0.43$ atoms $\mathrm{g} \mathrm{a}^{-1}$ (later updated to $4.49 \pm 0.39$ atoms $\mathrm{g} \mathrm{a}^{-1}$ ) and $30.2 \pm 2.6$ atoms $\mathrm{g} \mathrm{a}^{-1}$ for ${ }^{10} \mathrm{Be}$ and ${ }^{26} \mathrm{Al}$ from spallogenic reactions (Balco et al., 2008). The production of ${ }^{10} \mathrm{Be}$ and ${ }^{26} \mathrm{Al}$ 


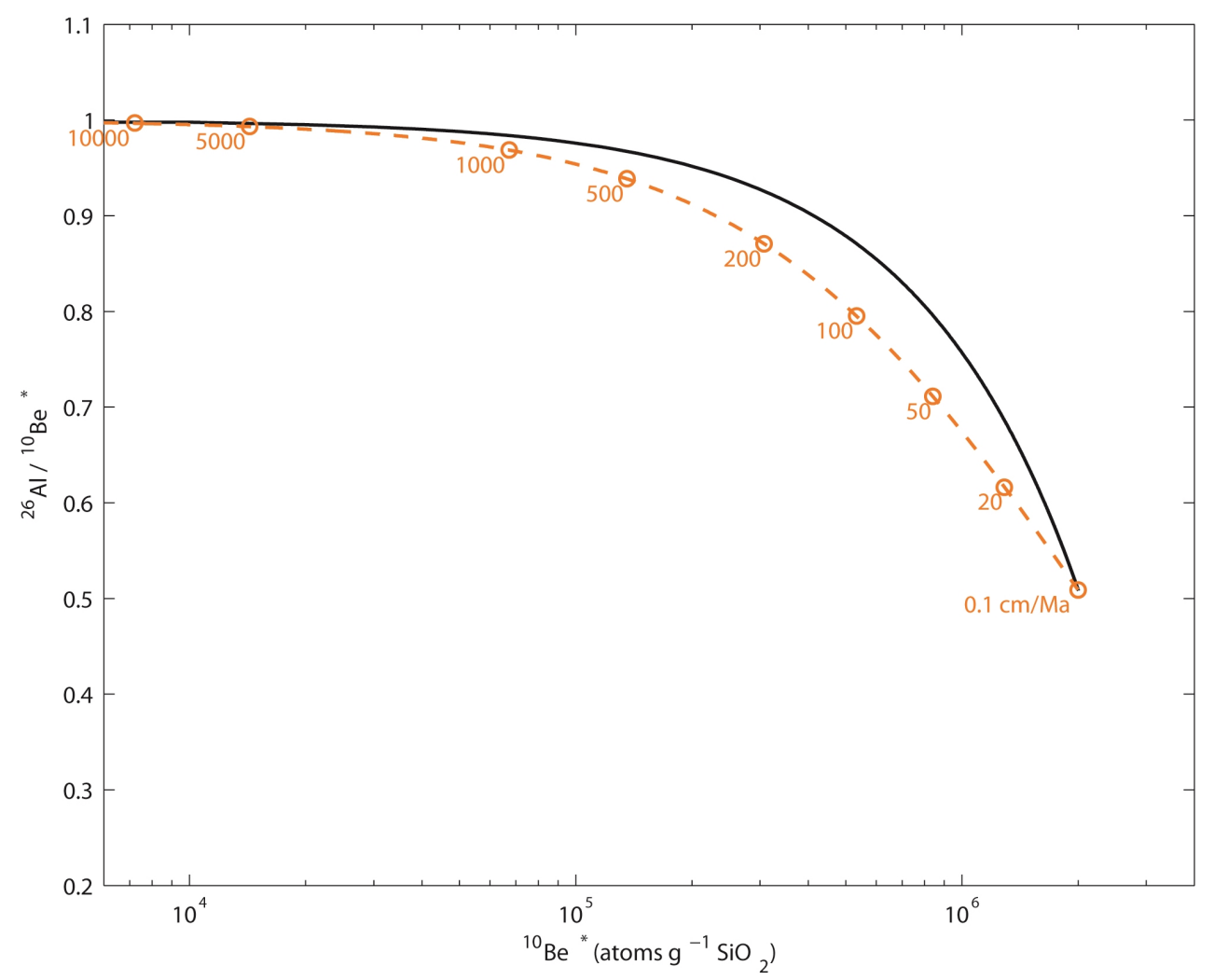

Figure 3.6 Nuclide concentrations over time with continuous exposure and steady-state erosion. Surfaces that are continuously exposed (shown here for $20 \mathrm{Ma}$ ) but that experience different degrees of constant subaerial erosion (shown for 0.1-10,000 $\mathrm{cm} / \mathrm{Ma}$ ), follow diverging trajectories towards individual end-points (orange), when saturation is reached. The area between the zero erosion (black; Figure 3.5) and erosion to saturation (dashed orange) lines is known as the 'steady-state erosion island' (Lal, 1991), and any surfaces with concentrations that occur in this area are assumed to be continuously exposed.

is assumed to vary equally through time. Therefore, the relatively well-constrained ${ }^{10} \mathrm{Be}$ production rate can be used to calculate the production rate of ${ }^{26} \mathrm{Al}$, using the relationship $P^{26} \mathrm{Al}=P_{{ }_{10} \mathrm{Be}} \times 6.1$ (later updated to 6.75) (Balco et al., 2008).

More recently, new calibration sites with improved precision and independent age control made scientists question the validity of a global ${ }^{10} \mathrm{Be}$ production rate. Importantly for Antarctica, new sites in the Southern Hemisphere and high-latitude Arctic indicate a production rate much lower than the global reference dataset. The production rate by spallation has been estimated in New Zealand as $3.88 \pm 0.10$ atoms $\mathrm{g} \mathrm{a}^{-1}$ (with Lal/Stone scaling; Putnam et al., 2010) and in South America as $3.70 \pm 0.11$ atoms $\mathrm{g} \mathrm{a}^{-1}$ (Lal/Stone

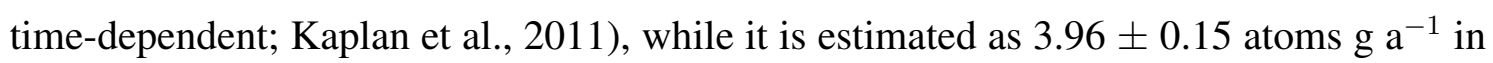
the Arctic (Lal/Stone; Young et al., 2013). These recent estimates overlap within 1-sigma uncertainty, suggesting a global production rate closer to $\sim 3.85$ atoms $\mathrm{g} \mathrm{a}^{-1}$. However, no calibration sites currently exist in Antarctica and the closest sites lie approximately 


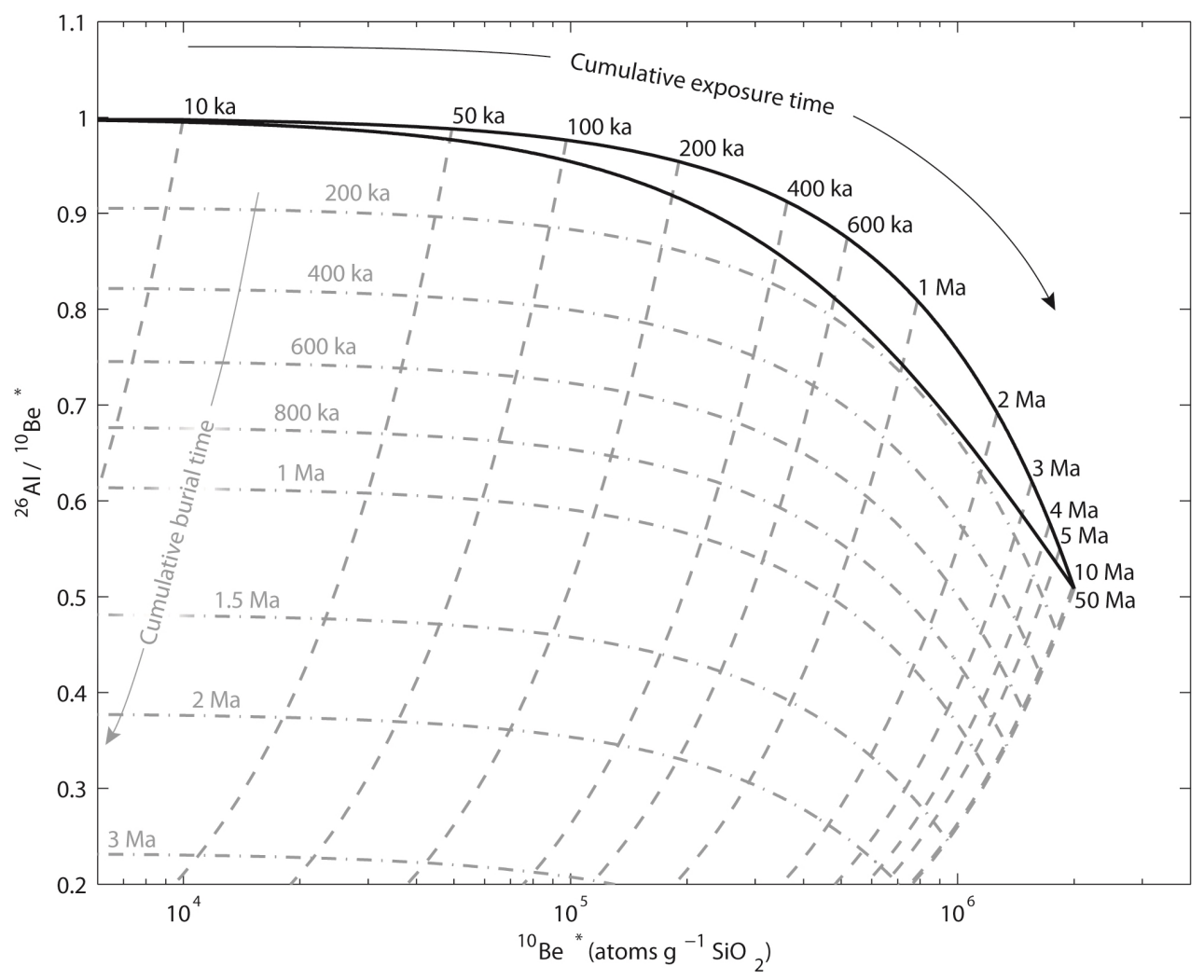

Figure 3.7 Nuclide concentrations over time with exposure and burial isochrons. If the concentrations of a surface fail to plot within the steady-state erosion island (Figure 3.6), then the surface has experienced burial. Isochrons represent lines of equal burial (dot-dashed) and exposure (dashed).

$25-30^{\circ}(3000-4000 \mathrm{~km})$ to the north. Therefore, the determination of ${ }^{10} \mathrm{Be}$ and ${ }^{26} \mathrm{Al}$ production in Antarctica still relies heavily on latitudinal scaling.

Several scaling schemes have been developed to account for the latitudinal variation of incoming cosmic rays and subsequent nuclide production. Some of these scaling models focus on the non-dipole nature of the geomagnetic field, based largely on grounded surveys of cosmic-ray intensities (cf. Granger et al., 2013). Dunai (2000) recognised variations in neutrons across latitudes and altitudes, and established a model that scaled nuclide production accordingly. Desilets and Zreda (2003) developed an alternative scheme based on the same spatially variable effects but with a different survey dataset, which was later modified (Desilets et al., 2006). Lifton et al. (2005) further adapted this approach, and amalgamated a longer observational dataset of neutron monitor surveys and integrated solar modulation effects. These methods produced very similar results to each other, but importantly for Antarctica, accounted for large deviations at lower latitudes rather than at the poles.

The simplest of existing scaling schemes happens to be the most appropriate for Antarc- 
tica. Stone (2000) recognised that the Antarctic ice sheet affected the atmosphere causing persistently lower air pressures (by 20-40 hPa) than elsewhere on Earth (Figure 3.8). This results in higher production rates. By modifying the early latitude-altitude scaling of Lal (1991), Stone (2000) replaced altitude with weather station derived Antarctic air pressure (Radok et al., 1996) to determine production rate scaling suitable for both the ice sheet and Transantarctic Mountain areas. This scheme also accounted for the reduced attenuation of muons in the atmosphere with a separate scaling factor to that of spallogenic nuclide production.

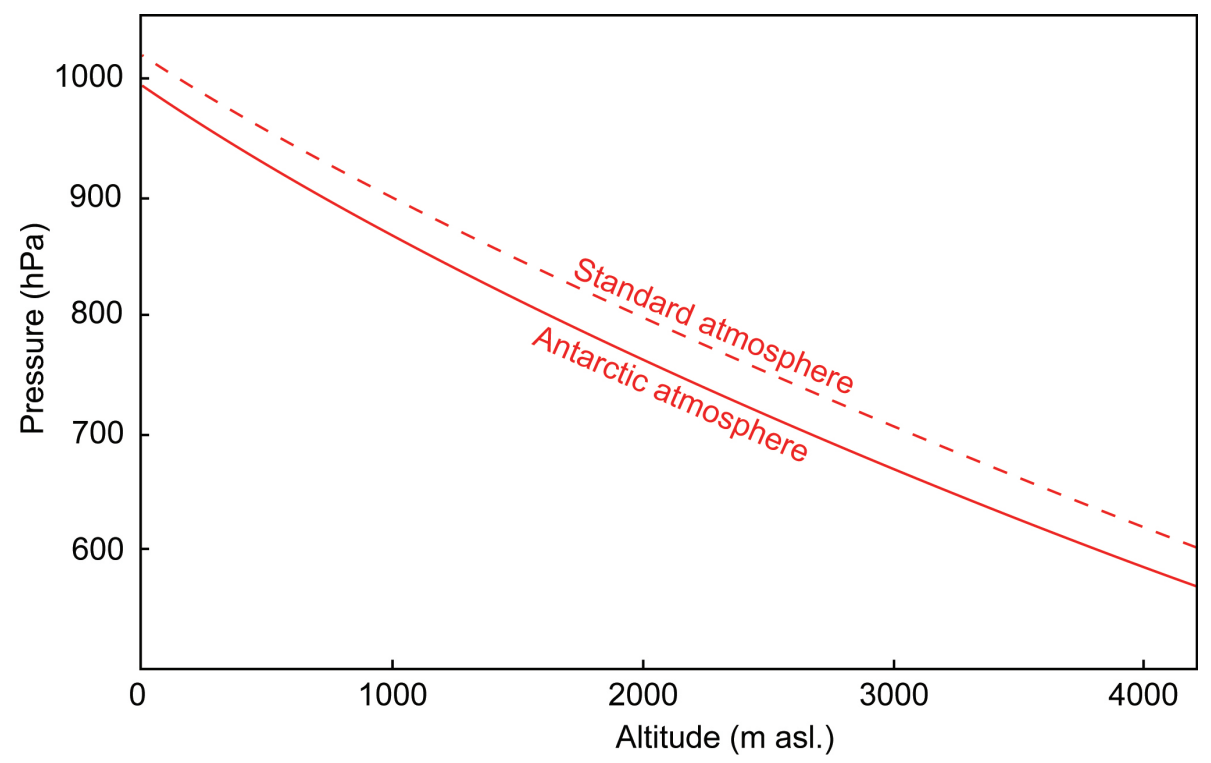

Figure 3.8 Atmospheric pressure-altitude relationship in Antarctica compared to the standard atmosphere (after Stone, 2000). Curves are based on measured long-term air pressures, which are from land sites at $>60^{\circ} \mathrm{S}$ latitude in Antarctica.

Production rates at the Earth's surface do not only vary spatially today, but likely varied through time due to changes in the magnetic field, and also in the intensity of the galactic source. Calibration sites span most of the last $\sim 30 \mathrm{ka}$ and are largely inclusive of any production rate changes over this time, however uncertainty greatly increases beyond this period. Temporal scaling requires past reconstructions of the magnetic field, which are applied as a function of the geomagnetic cutoff-rigidity (Balco et al., 2008). Several schemes include such scaling (Dunai, 2001; Desilets et al., 2006; Lifton et al., 2005), with Lifton et al. (2005) additionally accounting for temporal variations in solar activity. However, any changes in cosmic ray palaeo-intensity only need to be corrected for at latitudes below $60^{\circ}$ (Desilets and Zreda, 2003; Pigati and Lifton, 2004), as geomagnetic field lines at the poles are near-vertical and therefore any variations in field strength have little affect on cosmic ray flux. Chapter 5 provides further discussion regarding long-term production rate variation through time. 
Additional temporal variations of a surface's production rate can occur from changes in altitude at a site. These occur principally through tectonic activity and glacio-isostatic adjustments. In the Transantarctic Mountains, most uplift occurred prior to the Late Cenozioc (Elliot, 2013), however exact timings and rates of uplift events are not well understood. During glacial maxima, isostatic depression occurred around the fringes of Antarctica (e.g. Whitehouse et al., 2012), however, rock surfaces were covered by ice, inhibiting nuclide production. Subsequent deglaciation during interglacials would have caused delayed uplift (e.g. $32 \mathrm{~m}$ during the Holocene; Hall et al., 2004), with resulting enhanced nuclide production. While difficult to correct for in most Antarctic locations, such temporary isostatic uplift responses would only affect site-specific production rates and corresponding mean exposure ages within uncertainty bounds (Figure 3.9).

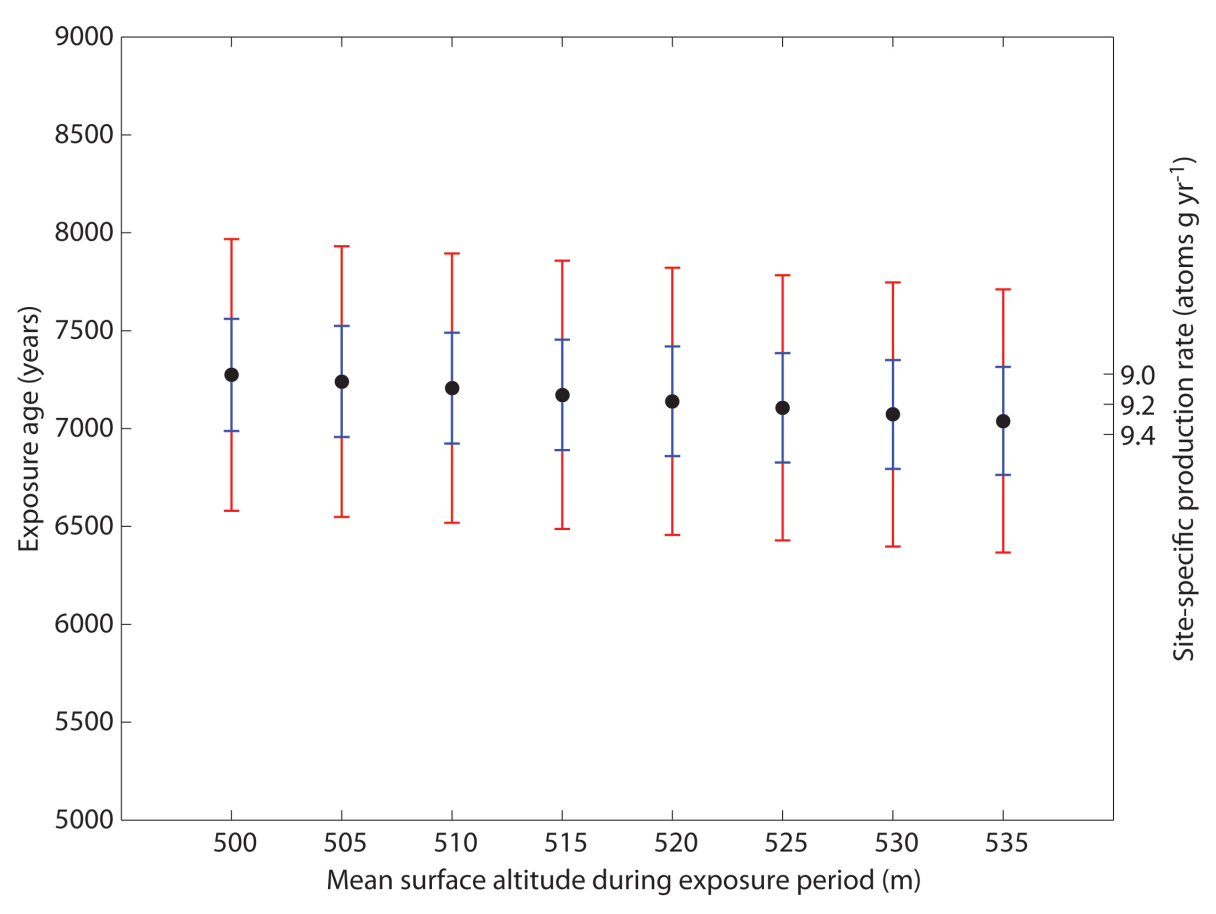

Figure 3.9 Scenario of changing production rate and exposure age with altitude. Using a hypothetical ${ }^{10} \mathrm{Be}$ concentration at a location of recorded past isostatic uplift (Hall et al., 2004) and a series of mean altitudes from $500 \mathrm{~m}$ above modern sea level, site production rates and corresponding exposure ages were calculated during the Holocene (black circles). Site-specific production rates are shown to change by $\sim 0.12 \%$ per metre elevation change. Over this time, a mean altitude change of $35 \mathrm{~m}$ affects mean exposure ages only within internal uncertainties (blue error bars). In this scenario, only after $85 \mathrm{~m}$ change in mean altitude do internal uncertainties become distinguishable. External age uncertainties are also shown (red error bars). For explanations of 'internal' verses 'external' uncertainties please see section 3.2. 


\subsection{Surface exposure dating}

Past geomorphic events associated with the evolving surface of a landscape, whether driven by climatic or tectonic processes, can be quantified using surface exposure dating. As previously discussed, cosmogenic nuclides can accumulate in a wide selection of lithologies, covering an age range of $10^{2}$ to $10^{7}$ years. Since initial developments of the technique (e.g. Klein et al., 1986; Lal, 1988), improvements in nuclide measurement and production rate estimates have enabled its effective application across a swath of environments, from wave-cut platforms to alluvial fans, landslides to volcanic eruptions, and highly faulted to highly stable land surfaces (reviewed in Ivy-Ochs and Kober, 2008; Dunai, 2010; Granger et al., 2013; Darvill, 2013). In particular, surface exposure dating has provided a major contribution to the understanding of glacial processes and past glacial extents, improving our knowledge of past climate (Balco, 2011).

In Antarctica, surface exposure dating provides the primary age control for terrestrial landscape evolution and ice sheet fluctuations, for three main reasons: 1) Its applicability across a range of lithologies and time spans supersedes alternative techniques bettersuited to temperate settings; 2) Substantial continental ice cover increases the chances of erratic cobbles being subglacially-derived prior to exposure, thereby reducing the chance of inheritance of cosmogenic nuclides; 3) Antarctica's climate reduces the possibility of post-depositional disturbance. Each of these reasons will now be discussed.

Early Antarctic glacial chronologies commonly used radiocarbon dating, however this approach requires organic material that is scarcely found on and around the continent. Previously, radiocarbon dating has targeted shells, forams, diatoms and bulk organic sediment in marine environments (e.g. Licht et al., 1996; Domack et al., 1999), and algae and faunal remains onshore (e.g. Bockheim et al., 1989; Hall and Denton, 2000; Hall et al., 2004). Many of these organic deposits sourced their radiocarbon from the ocean, which can accommodate 'old', excess radiocarbon, while some bioturbated sediments can recycle 'old' carbon. The top of offshore sediment cores record the offset between actual (modern) and radiocarbon-determined ages, and in the Ross Sea region corrections ranging from $1.1 \mathrm{ka}$ to >1.4 ka are required (Hall et al., 2010; Anderson et al., 2014). As surface exposure dating does not rely on such organic material, it is more suitable to dating terrestrial landforms and surface evolution.

The exposure age of a rock surface is dependent on whether the surface has an inherited exposure signal and whether it has maintained consistent nuclide production since exposure. Very few areas of Antarctica are ice-free and therefore erratic cobbles were likely eroded subglacially before being transported to the glacier flanks, in which case previous exposure of the cobble to cosmic rays less likely. 
Post-depositional processes can affect the nuclide concentration, however these effects are minimised in the cold, arid Antarctic climate. Subaerial weathering rates are extremely low (e.g. $0.16 \mathrm{~mm} \mathrm{ka}^{-1}$ in East Antarctica (Fink et al., 2006), $0.7 \mathrm{~mm} \mathrm{ka}^{-1}$ in the Dry Valleys (Ivy-Ochs et al., 1995)), and snow cover is typically limited and short-lived. The relative inactivity of surface processes means that burial and remobilisation of glacial deposits is less likely than in a temperate environment.

\subsubsection{Cosmogenic nuclide inheritance and other sources of error}

Despite surface exposure dating being well-suited to the terrestrial, ice-free areas of Antarctica, the cold environment can hamper reliable, internally-consistent chronologies. Antarctica's ice sheets are predominantly cold-based (Pattyn, 2010), which reduces the potential of subglacial erosion (e.g. Jamieson et al., 2010; Golledge et al., 2013). If less than $\sim 2.5 \mathrm{~m}$ of rock is eroded during periods of glacier expansion, then the rock surface can contain inherited cosmogenic nuclides, accumulated during multiple periods of prior exposure. In this situation, the nuclide concentration accumulated at the rock surface is artificially increased, causing an apparent age overestimation since last covered by ice. Such 'complex' exposure histories have been recorded in West Antarctica (e.g. Sugden et al., 2005), East Antarctica (e.g. Hein et al., 2014), the Antarctic Peninsula (e.g. Bentley et al., 2006) and in the Transantarctic Mountains (e.g. Storey et al., 2010). Bedrock surfaces are more likely to retain inherited cosmogenic nuclides as cobbles and boulders are mainly derived subglacially.

Clasts can be recycled during successive glacial periods, resulting in a scattered population of surface-exposure ages. Interpreting such a population of exposure ages depends on the complexity of the depositional setting. Previously, the youngest erratic clasts have been taken as the true ages of most recent exposure (e.g. Stone et al., 2003; Mackintosh et al., 2007; Bentley et al., 2010), with older ages assumed to be anomalies that reflect complex exposure histories. In blue-ice ablation areas, erratic clasts can record a scattered exposure history from nuclide accumulation prior to the clast being deposited (Fogwill et al., 2012). However, on the ice-free flanks of a glacier, where material has been deposited and weathered over multiple glacial cycles, the oldest exposure age would best reflect the timing of initial deposition (Swanger et al., 2011). In an attempt to better understand the scatter of exposure ages, statistical assessment of a dataset (Hein et al., 2014) or numerical modelling of complex exposure histories (Lilly et al., 2010) can be applied. However, by carefully considering the subtle but identifiable signs of cold-based glacier activity (e.g. Atkins et al., 2002; Joy et al., 2014) in the field, it may possible to obtain an internally-consistent chronology free of scatter. 
While shielding from cosmic rays by sediment or snow cover may be relatively small, its occurrence is hard to constrain over the total period of exposure. In depositional environments where sediment is able to cover cobbles or bedrock (e.g. from rockfall or windblown transport), or where till cover is deflated or eroded away, the true exposure age can be underestimated. The situation is similar for snow cover, however the lower density of snow compared to sediment or rock means that it is less of a concern; several tens of metres of snow cover is required to fully attenuate radiation (Zweck et al., 2013; Delunel et al., 2014), therefore preventing nuclide production in the underlying rock surface. By strictly following a robust sampling strategy, such burial effects can be avoided.

\subsubsection{Sampling strategy}

In this thesis, I aim to record the exposure of rock surfaces that results from lowering of the ice sheet surface. Ackert et al. (1999) were the first to use surface exposure dating to identify Antarctic ice sheet surface lowering from an LGM maximum thickness. Later, more continuous records of ice sheet thinning were reconstructed (Stone et al., 2003) and glacial-interglacial ice sheet fluctuations were investigated (Lilly et al., 2010).

Here, sampling sites are targeted based on certain, chapter-specific objectives. In Chapter 5, I seek to record potential glacial-interglacial changes of ice surface elevation using samples collected from nunataks distributed across the catchment. While in Chapter 7 , I look to obtain an LGM-to-present thinning history using a "dipstick" approach (Stone et al., 2003); glacial erratics are collected in altitudinal transects to record time-dependent exposure that results from transient ice surface lowering. In both studies, a prior assessment of the glacial system is made to evaluate the suitability of applying surface exposure dating (Chapters 4 and 6, respectively). Geomorphological evidence that indicates glacial erosion (e.g. Möller, 1995; Sugden and Denton, 2004) and deposition (e.g. Hall and Denton, 1999; Hall et al., 2000) is recorded in this region through the Late Cenozoic and during the LGM, indicating that suitable rock surfaces exist for the application of surface exposure dating in this thesis.

In order to obtain a glacial chronology free of potentially spurious surface-exposure ages, I selected samples with the following preference: 1) erratic cobbles or boulders that show signs of glacial transport, as indicated by an exotic lithology and/or a glaciated surface (e.g. polishing, faceting and abrasions), resting on glacially-eroded stable bedrock, preferably on ridge crests so that prior mobilisation can be ruled out; 2) cobbles resting on com- 
pacted surficial material likely deposited by glacier ablation, rather than clasts embedded in or near cryoturbation features such as patterned ground, which would indicate probable repeated burial; 3) bedrock surfaces with evidence of fresh and unweathered glacial erosion (e.g. streamlined, polished and striated). The specific sampling strategy used for each study is detailed in the respective chapter.

\subsubsection{Sample preparation, geochemistry and AMS measurement}

Preparation of samples for surface exposure dating was conducted at the sedimentology, cosmogenic nuclide and geochemistry laboratories of Victoria University of Wellington (VUW) and GNS Science, New Zealand. Details of the procedures can be found in Appendix $\mathrm{A}$ and $\mathrm{B}$.

The upper $\sim 2-8 \mathrm{~cm}$ of each sample was extracted for processing using a large circular saw. This material was then crushed with a fine jaw-crusher and sieved to retain sand-sized grains (250-500 microns). Each sample was initially cleaned using a Frantz Isodynamic separator (at $\sim 0.5$ Amps and a $10^{\circ}$ tilt) to remove the magnetic component. Further cleaning was required to remove non-quartz minerals and etch any meteoric beryllium from the outer surface of the grains. This was achieved for each sample (up to $150 \mathrm{~g}$ ) with a 1-day leach in hydrochloric acid and then three 2-day leaches in a 5\% mixture of weak hydrofluoric acid and nitric acid, warmed and rotated on hotdog rollers. A final 1-hour hydrofluoric acid (7 M) leach and concentrated aqua regia cleaning was carried out to remove any remaining meteoric beryllium prior to sample dissolution and isotope extraction.

Beryllium and aluminium were extracted following established geochemical procedures (von Blanckenburg et al., 1996, 2004; Norton et al., 2008). A ${ }^{9}$ Be spike ( 0.15-0.18 mg per sample; $375 \mathrm{ppm}$ ) was added to the quartz samples, which were then dissolved in concentrated hydrofluoric acid. Anion exchange columns were first used to remove iron, and then cation exchange columns were used to isolate beryllium from sodium, magnesium, and the aluminium. An additional anion column stage then enabled the separation of aluminium from the remaining titanium and iron. Precipitates were then either oxidised with silver, or oxidised and mixed niobium, externally.

Analytical measurements were undertaken at ETH Zurich (Switzerland) mass spectrometry facilities using both Tandem and Tandy accelerator mass spectrometers (Christl et al., 2013). For beryllium, all samples were measured relative to the ETH Zurich in-house 
standard S2007N (nominal ${ }^{10} \mathrm{Be} /{ }^{9} \mathrm{Be}$ ratio of $28.1 \pm 0.8 \times 10^{-12}$ ), which in turn was calibrated relative to the ICN 01-5-1 standard $\left({ }^{10} \mathrm{Be} /{ }^{9} \mathrm{Be}\right.$ ratio of $\left.27.09 \pm 0.3 \times 10^{-12}\right)$ (Nishiizumi et al., 2007), and were then corrected with procedural blanks. For aluminium, samples were measured relative to the in-house standard ZAL94N (nominal ${ }^{26} \mathrm{Al} / \mathrm{Al}$ ratio of $480 \times 10^{-12} \pm 3.8 \%$ ), subsequently normalised to the KN-01-4-1 standard (Nishiizumi, 2004). Meanwhile, stable aluminium was measured at VUW and GeoForschungsZentrum (GFZ) (Potsdam, Germany), corrected with a caesium spike and checked with standard addition.

\subsubsection{Exposure age determination}

Following the collection of samples and measurement of nuclide concentrations, exposure ages can be determined from the estimated rates of nuclide production and decay (Eq. 3.1 and 3.2). In this thesis, ages are determined using the CRONUS-Earth calculator and procedures (Balco et al., 2008), which provides a 'best practice' approach and common basis for all surface-exposure dating studies.

Here, all exposure ages are calculated using a range of production rate scaling schemes (discussed previously), but using Antarctic-specific atmospheric pressure (Stone, 2000). The global dataset production rate (Balco et al., 2008) is primarily used, however exposure ages derived from the New Zealand based production rate are additionally evaluated. Also required for determination of the production rate at the sample site are the longitude, latitude and altitude. Topographic shielding, measured in the field, is included as a calculated shielding factor. Subaerial weathering is considered small and uncertain, and is therefore assumed to be zero.

The CRONUS-Earth approach seeks to include the uncertainties from nuclide measurements, scaling schemes as well as all input parameters to the scaling schemes (Balco et al., 2008). Both 'internal' and 'external' uncertainties are provided as an output. The internal uncertainty includes solely the measurement uncertainty of the nuclide concentration, and is intended for comparing sample measurements from a single study area or feature. External uncertainties additionally include production rate uncertainty from both spallation and muons. Comparison between surface exposure ages in this thesis and other (cosmogenic or radiocarbon) chronologies uses the external uncertainties. The largest uncertainty, however, is the choice of reference production rate, which is not accounted for in either the internal or external uncertainty; in Antarctica, a mid-Holocene mean exposure age would differ by $\sim 1,000$ years depending on whether the global or New Zealand 
calibration dataset is used. Production rate uncertainty is discussed in the thesis where relevant to chronological interpretations.

\subsection{Glaciological modelling}

\subsubsection{One-dimensional flowline model approach}

Glacier flowline modelling (e.g. Oerlemans, 1997) is applied in this thesis to provide both a quantitative evaluation of the outlet system and investigate past glacier dynamics in combination with the surface-exposure chronology. Flowline models, unlike continental-scale ice sheet models, are less complex and therefore can be applied at higher spatial resolutions and are especially suited to understanding along-flow dynamics and mechanisms of advance/retreat. Here, I use a one-dimensional (1-d) finite difference flowline model, which has previously been applied to Transantarctic Mountain (Golledge and Levy, 2011; Golledge et al., 2014a) and other Antarctic glaciers (Davies et al., 2014).

The modelling approach used here has two main stages. Firstly, empirically-constrained modern glacier surface and velocity profiles are simulated to steady-state. This ensures that uncertain flow and environmental variables are correctly tuned to the observed ('known') glacier configuration. Secondly, past glacier profiles are simulated, constrained by the new surface exposure data and/or palaeoclimate and geological data in the region. The nature of these simulations is dependent on the study objectives; Chapter 4 compares the past steady-state regimes of Skelton Glacier, while Chapter 7 investigates the transient response of Mackay Glacier from an advanced steady-state configuration. Sensitivity tests are additionally performed in order to identify the potential response of a glacier to a range of possible environmental and flow variables.

\subsubsection{Numerical model formulation}

Ice thickness in the model evolves over the grounded portion of the glacier, using a forward explicit numerical scheme. The change in ice thickness is calculated using the mass conservation equation (Cuffey and Paterson, 2010):

$$
\frac{\partial H}{\partial t}=M-\nabla \cdot q
$$


where $H$ is ice thickness (metres), $t$ is time (years), $M$ is net accumulation ( $\mathrm{m} \mathrm{a}^{-1}$ ) and $\nabla \cdot q$ is the gradient of ice flux. Steady-state is reached when the gradient of ice flux balances net accumulation. This equation is integrated through time using an adaptive time-stepping scheme (Hindmarsh, 2001), which is a function of grid size and peak ice flux (Golledge and Levy, 2011).

The 1-d model implements this change in ice thickness along the flowline from the centreline velocity $\left(u, \mathrm{~m} \mathrm{a}^{-1}\right)$ and symmetrical trapezoidal cross-sectional area $(\gamma)$, which is determined from the valley-side angle and valley width (e.g. Oerlemans, 1997), for each cell down-glacier $(d x)$ as follows:

$$
\frac{\partial \gamma}{\partial t}=\frac{\partial}{d x}(\gamma u)+M
$$

The shallow-ice approximation is used together with a longitudinal averaging scheme to calculate basal shear stress $\left(\tau_{b}, \mathrm{kPa}\right)$ along the flowline. This effectively smooths out large along-flow variability in velocity and gravitational driving stresses (Kamb and Echelmeyer, 1986; Echelmeyer and Kamb, 1986):

$$
\tau_{b}=\frac{1}{2 \ell H^{1 / n}} \int \tau_{d} F H^{1 / n} e^{-|\Delta x| \ell} d x
$$

in which $\ell$ represents the longitudinal coupling length (metres), $F$ is a width-dependent shape factor, $\Delta x$ is the horizontal cell size (metres), and driving stress $\left(\tau_{d}, \mathrm{kPa}\right)$ is derived from:

$$
\tau_{d}=-\frac{\partial S}{\partial x} \rho g H
$$

where $\partial S / \partial x$ is the glacier's surface slope, $\rho$ is the density of ice $\left(920 \mathrm{k} \mathrm{g} \mathrm{m}^{3}\right)$ and $g$ is gravity $\left(9.81 \mathrm{~m} \mathrm{~s}^{2}\right)$.

Centreline surface velocities $(u)$ are calculated from both basal sliding and deformation components:

$$
u=f_{d} H \tau_{b}^{n}+f_{s} \frac{\tau_{b}^{p}}{H}
$$

where $f_{d}$ and $f_{s}$ represent the deformation $\left(\mathrm{kPa}^{-3} \mathrm{a}^{-1}\right)$ and sliding $\left(\mathrm{Pa} \mathrm{m}^{2} \mathrm{a}^{-1}\right)$ parameters 
respectively, $n$ is Glen's flow law exponent (3) and $p$ is the sliding exponent (3).

The deformation rate factor is temperature-dependent (Cuffey and Paterson, 2010). Energy required for creep activation $(Q)$ is $60 \mathrm{~kJ} \mathrm{~mol}^{-1}$ below and $139 \mathrm{~kJ} \mathrm{~mol}^{-1}$ above $263.15 \mathrm{~K}$, whereby the thermal parameter $(\xi)$ is $1.14 \times 10^{-5}$ and $5.47 \times 10^{10}$ respectively (Hubbard, 2006). Using these values, the deformation parameter is determined as follows:

$$
f_{d}=2 A_{0}(n+1)^{-1}
$$

where

$$
A_{0}=E \xi \exp \left(\frac{-Q}{R \bar{T}}\right)
$$

in which $R$ represents the universal gas constant $\left(8.314 \mathrm{~J} \mathrm{~mol}^{-1} \mathrm{~K}^{-1}\right), \bar{T}$ is the columnaveraged ice temperature $(\mathrm{K})$, and $E$ is an enhancement coefficient, employed to account for softening of the ice by impurities. Based on observed strain rate relationships, suitable Antarctic values for enhancement are 2-10 (Cuffey and Paterson, 2010).

Glacier motion by basal sliding occurs with the production of melt at the bed, once the pressure-melting point is reached. As sliding can still occur in sub-freezing temperatures (e.g. Echelmeyer and Zhongxiang, 1987; Cuffey et al., 1999), an exponential function is used to enable sliding over a range of temperatures near to the pressure-melting point (Hindmarsh and Le Meur, 2001):

$$
f_{s}=f_{s 0} \exp \left[-1 \times\left(\left(8.7 \times 10^{-4} H\right)-T_{b}\right)\right] .
$$

The degree of sliding can vary spatially along the glacier depending on possible influxes of meltwater (e.g. Stearns et al., 2008) and transitions to a more/less deformable bed (e.g. Kamb, 2001). Here, a spatially variable basal sliding coefficient $\left(f_{s 0}\right)$ is used where there is evidence of a major along-glacier change in traction at the bed (i.e. Chapter 7, where the marine portion has softer, more deformable sediments). Flow of the glacier is primarily controlled by the enhancement and sliding coefficients, which are tuned to most closely simulate steady-state configurations.

Net mass balance $(M)$ is integral to the calculation of ice thickness continuity through time (Eq. 3.4 and 3.5). The model uses a positive degree-day scheme to calculate surface melting. In Antarctica, the air temperature predominantly remains below $0^{\circ} \mathrm{C}$, at least in the present-day climate. This dry, high-radiation climate facilitates sublimation, which 
consumes high amounts of energy. As a result, the remaining energy for melt is much reduced, and the corresponding degree-day factor is low (Hock, 2003). In common with other Antarctic studies, a degree-day factor $\left(F_{p d d}\right)$ between 0.001 and $0.01 \mathrm{~mm} \mathrm{~K}^{-1} \mathrm{~d}^{-1}$ is used in the model (Huybrechts and Oerlemans, 1990; Davies et al., 2014). The scheme is implemented if the monthly mean ice surface temperature $\left(T_{s}^{m}, \mathrm{~K}\right)$, determined from a cosine function of a mean annual air temperature (Golledge and Levy, 2011), is $\geq 0$ :

$$
M=\sum_{m=1}^{12} P_{s}^{m}-\left(T_{s}^{m} \times F_{p d d}\right)
$$

where $m$ is the month of the year, $P_{s}^{m}$ is the effective monthly precipitation at the glacier surface $\left(\mathrm{m} \mathrm{a}^{-1}\right)$ and $F_{p d d}$ equals $0.005 \mathrm{~mm} \mathrm{~K}^{-1} \mathrm{~d}^{-1}$. Annual precipitation is assumed to equal surface accumulation where $T_{s}^{m} \leq 0$, whereas above this temperature, precipitation is assumed to be liquid and thus not retained by the glacier (effective precipitation, $P=$ $0)$. Precipitation varies according to elevation using an adjustable lapse rate from sea level $\left(\mathrm{m} \mathrm{a}^{-1} \mathrm{~km}^{-1}\right)$ as well as distributed equally throughout the year, and the model therefore ignores any additional spatial and temporal variability (e.g. Morse et al., 1998).

Mass loss in Antarctica primarily occurs at the terminus, dominantly via calving and sub-glacier melt. Detailed assessments of ice dynamics across the grounding-line (e.g. Schoof, 2007) and of the associated downstream stress regime should use the shallow shelf approximation (e.g. MacAyeal, 1989), however, in this thesis I am primarily concerned with simple changes in ice thickness of the grounded portion of outlet glaciers. Although imperfect, negative mass balance effects of both the grounded and floating portions included in the model are determined by the relationship between ice thickness at the terminal cell and the floatation threshold:

$$
H \leq\left(\psi-\left[b+H_{\varphi}\right]\right) \frac{\rho_{w}}{\rho}
$$

in which $\psi$ is sea level (metres), $H_{\varphi}$ is the subglacial till layer thickness (metres), $\rho_{w}$ is the density of seawater (1028 $\mathrm{kg} \mathrm{m}^{-3}$ ), and $b$ is the bed elevation (metres), adjusted through time in response to isostatic (un)loading (Golledge and Levy, 2011).

For ice thickness below this threshold the cell is effectively floating, and mass loss is calculated following an empirically-derived sub-shelf melt relationship with ocean temperature $\left(T_{o},{ }^{\circ} \mathrm{C}\right)$ (Holland et al., 2008):

$$
M_{s h}=M-0.341 T_{o}^{2}+2.365 T_{o}+3.003 .
$$


Whereas, for ice thickness above this threshold, loss occurs through calving at the grounded terminal cell (Cuffey and Paterson, 2010):

$$
M_{c v}=M-\eta(c[\psi-b])
$$

where $\eta$ is a scalable coefficient and $c$ is a constant (17). The parameters $T_{o}$ and $\eta$ are used for tuning purposes.

Temperature at the glacier's surface heavily influences the basal ice temperature, achieved through the thermal conductivity of ice $\left(\kappa_{i c e}, 2.4 \mathrm{Wm}^{-1} \mathrm{~K}^{-1}\right)$. Together with contributions from an upward geothermal heat flux $\left(G, \mathrm{~mW} \mathrm{~m}^{2}\right)$, sliding-derived strain heating and both vertical and horizontal advections of heat (Cuffey and Paterson, 2010), a pressure-melting adjusted basal ice temperature $\left(T_{b}, \mathrm{~K}\right)$ is calculated (Hindmarsh and Le Meur, 2001):

$$
T_{b}=T_{s}+\left(\frac{G H}{\kappa_{i c e}}\right)+\left(8.7 \times 10^{-4} H\right)-\left(u \frac{\partial T}{\partial x}+w \frac{\partial T}{\partial z}\right)+\left(2 \frac{\partial \bar{u}}{\partial x} \tau_{b}\right)+\left(\tau_{b} u_{s}\right)
$$

where $x$ is the horizontal distance along the flowline (metres), $z$ is the vertical position in the ice column (metres), and $w$ and $u$ are the vertical and horizontal velocities $\left(\mathrm{m} \mathrm{a}^{-1}\right)$.

Complexities in the vertical profiles of ice temperature and flow are simplified with a linear temperature gradient and vertically-averaged flow. Furthermore, the use of a flow rate enhancement coefficient (Eq. 3.10) reduces potential velocity errors derived from the determination of ice temperatures.

Although subglacial erosion is considered to be minor in the Transantarctic Mountains (e.g. Cuffey et al., 2000), the model used here allows for the investigation of bedrock erosion and associated till flux effects. The presence and magnitude of subglacial erosion $\left(\varsigma, \mathrm{m} \mathrm{a}^{-1}\right)$ depends on temperature and pressure conditions at the bed, and is calculated using basal shear stress and sliding velocity (Pollard and DeConto, 2003):

$$
\varsigma=0.2 \times 10^{-9} \tau_{b} u_{s}\left(1-\frac{H_{\varphi}}{\varphi_{\max }}\right)
$$

in which $H_{\varphi}$ represents the subglacial till layer thickness and $\varphi_{\max }$ represents an erosionlimiting till thickness (metres). Both bedrock erosion (till generation) and till flux are dependent on the thickness of the till layer and its coupling with the overriding ice; this 
model assumes perfect ice-till coupling and limits till thickness to $2 \mathrm{~m}$ based on observations (Golledge and Levy, 2011). While the magnitude of erosion is influenced by these assumptions (Fowler, 2000), the estimations calculated using this approach are sufficient for comparing relative changes in basal conditions down-glacier and that are derived from contrasting climatic regimes (i.e. Chapter 4).

\subsubsection{Boundary conditions and environmental forcing}

The flowline model is initialised for Skelton and Mackay Glaciers using present-day topographic and ice thickness data. Continental-scale ice sheet models (e.g. Pollard and DeConto, 2009; Golledge et al., 2014b) use gridded datasets of ice surface elevation, ice thickness and bed topography, such as BEDMAP2, where data from numerous aeriallyderived radar profiles across Antarctica are compiled (Fretwell et al., 2013). However, to overcome spatial gaps in the input data, large interpolations and a common grid resolution $(1 \mathrm{~km})$ are required. Glacier-scale flowline models often require these data with reduced uncertainties and a higher spatial resolution, and therefore I use local radar-derived ice depth data in this thesis where appropriate. At Skelton Glacier the ice surface and bed elevations were obtained from a CReSIS airborne-radar survey (Chapter 4), while at Mackay Glacier the ice surface was derived from an ICECAP airborne-radar survey and the bed topography was determined from BEDMAP2 data that was corrected in the lower reaches with ground-based radio-echoed ice thickness measurements (Calkin, 1974) (Chapter 7).

Model experiments are forced using climate parameterisations, which are either empiricallyderived constants or tuned variables. Values for the amount of precipitation, mean annual air temperature, precipitation and temperature lapse rates and annual temperature range are derived from local measurements (National Soil Survey / Landcare Research; Crary, 1966) and the Taylor Dome ice core at the head of the catchments (Morse et al., 1999; Steig et al., 2000), where possible. The near-surface ocean temperature is $-1.8^{\circ} \mathrm{C}$ in winter and $-1.6^{\circ} \mathrm{C}$ in summer, based on modern observations (Orsi and Whitworth, 2004). In situations where measurements are lacking or not considered representative of the system, the environmental variable is used as a tuning parameter (discussed in the respective chapters).

Time-dependent simulations are forced with regional-scale palaeoclimate data. Specifically, the Taylor Dome ice core (Steig et al., 1998; Monnin et al., 2004) is exploited to acquire values for past temperature and precipitation, while geological data is used to estimate past sea level (e.g. Dwyer and Chandler, 2009) and ocean temperature (e.g. Winter et al., 2012). Unconstrained or uncertain environmental variables are either used as tuning 
parameters or are assumed to be the same as today (e.g. temperature lapse rate).

\subsection{Summary}

This chapter has outlined the basis and application of the two main techniques employed in this thesis, surface-exposure dating and glaciological modelling. Surface-exposure dating is well-suited to Antarctica and is used here as both a geochronological and geomorphological tool, held with the responsibility of providing empirical evidence of past ice sheet fluctuations. While its fundamental physical basis is generally well understood, by far the largest limitation is an uncertain nuclide production rate in Antarctica. This uncertainty, however, does not significantly hamper the outcomes of this work, and provides an opportunity to better understand nuclide production through time. Glacier flowline modelling allows for a quantitative prediction of the along-glacier ice dynamics, particularly befitting to simple flow scenarios in mountainous settings. It is implemented here, in combination with surface exposure chronologies, to investigate the behaviour of Transantarctic Mountain outlet glaciers under different climatic and topographic conditions. 


\section{Chapter 4}

\section{Dynamics of Skelton Glacier under past and present climates}

\subsection{Introduction}

Understanding the dynamic responses of outlet glaciers has become more urgent following observations of termini retreat (Miles et al., 2013), dynamic thinning (Pritchard et al., 2009), and melt-induced ice-shelf collapse (Glasser and Scambos, 2008) and subsequent glacier acceleration (Scambos et al., 2004; Rignot et al., 2004). Antarctic glaciers are simulated to melt (Davies et al., 2014), increase discharge (Winkelmann et al., 2012) and possibly undergo non-linear grounding-line retreat (e.g. Favier et al., 2014; Mengel and Levermann, 2014) with projected atmospheric warming, however, the style and magnitude of such a response is heavily dependent on the glacial system (Oerlemans et al., 1998; Hulbe et al., 2008; Golledge et al., 2014a). This is most evident in the Transantarctic Mountains where catchments of different geometries and basal conditions can produce contrasting dynamic responses (Stearns et al., 2008; Golledge and Levy, 2011; Zoet et al., 2012; Golledge et al., 2014a). In this chapter, I investigate the dynamic behaviour of Skelton Glacier, a Transantarctic Mountain outlet of the East Antarctic Ice Sheet (EAIS), under past and present climates.

Since early theories of long-term East Antarctic ice sheet stability (e.g. Sugden et al., 1993), geological evidence and glaciological modelling have revealed new information regarding the time-dependent configurations and dynamics of outlet glaciers in this region. Ice sheet reconstructions from mapped glacial deposits indicate that a more extensive Antarctic ice sheet existed at the Last Glacial Maximum (LGM), which buttressed Transantarctic Mountain outlets that flowed into the Ross Embayment, causing them to thicken in their lower reaches (e.g. Bockheim et al., 1989; Denton and Hughes, 2000). Similar expansive ice sheet configurations are also suggested for the Pliocene, but with high amplitude fluctuations between fully glacial and interglacial states (Naish et al., 2009; Pollard and DeConto, 2009). In addition to changes in glacial extent, the evolving climatic conditions led to changes in the basal regime and erosive potential of outlet glaciers (e.g. McKay et al., 2009; Jamieson et al., 2010; Golledge et al., 2013). Considering the dramatic shifts in glacier behaviour inferred by these studies, the sensitivity of such systems to environmental forcings or contrasting steady-state climates is not well understood. 
Glaciological modelling of individual Transantarctic Mountain outlet glaciers enables the quantification of dynamics and comparison between systems. Modelling studies indicate that these glaciers likely flow predominantly by internal deformation and respond along their length to environmental forcings (e.g. Johnson and Staiger, 2007; Kavanaugh and Cuffey, 2009; Golledge and Levy, 2011), however, the form of dynamic response varies depending on the boundary conditions of the glacier (Golledge et al., 2014a). It is therefore important to understand whether the present-day behaviour of Skelton Glacier is typical of Antarctic, and specifically Transantarctic Mountain, outlet glaciers, and also whether the dynamics of these glaciers remains similar through time.

Here, the dynamic behaviour of Skelton Glacier is investigated to better understand: 1) the extent to which vertical and lateral changes in catchment topography influence the flow of Skelton Glacier, and the controls on basal sliding along the glacier; 2) the sensitivity of Skelton Glacier to changes in environmental variables; 3) the dynamics of Skelton Glacier under past climates (LGM, Pliocene interglacial, Pliocene glacial), and variations in the extent and magnitude of basal erosion under these different climatic conditions.

In order to address these objectives, I investigate the boundary conditions, glacier geometry and flow regime of Skelton Glacier using available observational datasets and a one-dimensional (1-d) finite difference glacier flowline model (Golledge and Levy, 2011). The model is tuned to reproduce the modern glacier geometry and ice velocities, and to then provide a quantitative evaluation of flow characteristics, thermal regime and basal erosion along the length of the glacier. In addition to testing the sensitivity of the glacier under present-day (warm, interglacial) conditions, I also compare the flow regimes for past steady-state glacier configurations, during cold Quaternary (LGM), cold Pliocene, and warm Pliocene climates.

\subsubsection{Previous glaciological studies of Skelton Glacier}

Skelton Glacier (159-162 $\left.{ }^{\circ} \mathrm{E}, 78-79^{\circ} \mathrm{S}\right)$ flows from Taylor Dome at the edge of the EAIS plateau, through the Transantarctic Mountains and feeds into the Ross Ice Shelf (Figure 4.1). A catchment of approximately $6000 \mathrm{~km}^{2}$ drains a large névé to the south-west and steep cirque tributary glaciers on the sides of the Royal Society Range to the north-east. A line of three nunataks (Névé Nunatak, Halfway Nunatak and Clinker Bluff) divide these source areas, which both flow into a laterally confined inlet downstream of Clinker Bluff and the grounding-line.

During the International Geophysical Year (1957-59), a series of ice thickness, velocity and meteorological measurements were undertaken as part of a traverse of Skelton 


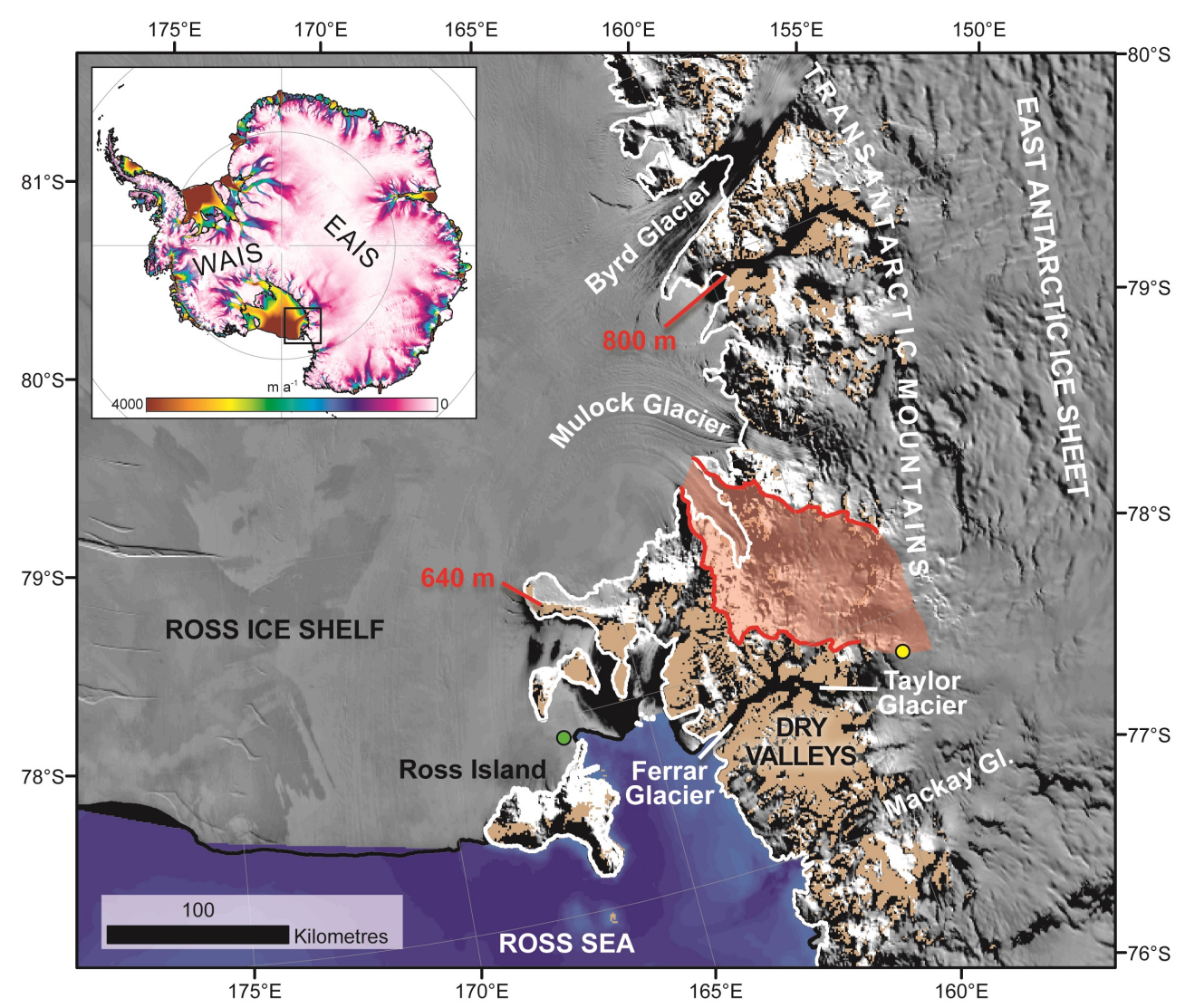

Figure 4.1 Geographic context of Skelton Glacier. Inset shows surface velocities of the West Antarctic (WAIS) and East Antarctic (EAIS) Ice Sheets, and the location of the main part of the figure. Skelton Glacier (red area) is sourced from the edge of the EAIS near Taylor Dome (yellow circle) and flows through the Transantarctic Mountains into the floating Ross Ice Shelf. At the LGM, grounded ice extended past the mouth of Skelton Glacier, with a surface sloping from $\sim 800 \mathrm{~m}$ asl (at Darwin-Hatherton Glacier; Anderson et al., 2004) to 640 m asl (at Minna Bluff; Denton and Marchant, 2000). Ice-free areas (brown), location of the ANDRILL core (green circle; Naish et al., 2009) and present-day grounding-line (white) are also shown.

Glacier. A seismic survey reveals an almost grounded, floating ice-shelf that extends 50 $\mathrm{km}$ from the grounding-line, through Skelton Inlet to the Ross Ice Shelf (Crary, 1966). At this confluence with the Ross Ice Shelf, the floating portion of Skelton Glacier was measured to be approximately $585 \pm 20 \mathrm{~m}$ thick (Crary, 1966; Swithinbank, 1969). Based on a velocity measurement of $0.28 \mathrm{~m} \mathrm{~d}^{-1}$ ( $\sim 102 \mathrm{~m} \mathrm{a}^{-1}$; Cameron and Goldthwait, 1961), an annual discharge of $\sim 0.8 \mathrm{~km}^{3}$ was calculated (Wilson and Crary, 1961). Elsewhere, ice surface velocities are recorded as $90 \mathrm{~m} \mathrm{a}^{-1}$ of the floating ice (Giovinetto et al., 1964), and $\sim 115$ to $350 \mathrm{~m} \mathrm{a}^{-1}$ (Golledge et al., 2014a; Humbert et al., 2005) at the grounding-line.

The spatial pattern of surface mass balance can be inferred from temperature and precipitation data. Borehole air temperatures measured just below the surface, which are typically $\pm 1{ }^{\circ} \mathrm{C}$ from the mean surface air temperature, changed near-linearly with alti- 
tude from $-21^{\circ} \mathrm{C}$ on the shelf $\left(84 \mathrm{~m}\right.$ asl) to $-42^{\circ} \mathrm{C}$ in the névé (2300 $\mathrm{m}$ asl) (Crary, 1966). Precipitation measurements range from 0.06 to $0.17 \mathrm{~m}$ w.e. $\mathrm{a}^{-1}$ at the Ross Ice Shelf confluence, and $0.11 \mathrm{~m}$ w.e. $\mathrm{a}^{-1}\left(2316 \mathrm{~m}\right.$ asl) to $0.36-0.4 \mathrm{~m}$ w.e. $\mathrm{a}^{-1}(800-2300 \mathrm{~m}$ asl) in the névé (Wilson and Crary, 1961; Crary, 1966). This suggests that accumulation at Skelton Glacier varies non-linearly with altitude, which is supported by climate reanalysis data that show local precipitation and surface mass balance that are heavily influenced by relief and a prevailing east-to-west precipitation gradient in this area (Lenaerts et al., 2012).

\subsection{Dynamic setting as inferred from observational datasets}

Airborne and satellite derived datasets allow an ice sheet to glacier scale assessment of surface morphology (Scambos et al., 2007), bed topography and ice thickness (Fretwell et al., 2013), mass balance (Rignot and Thomas, 2002) and ice surface velocity (Rignot et al., 2011). Using these datasets, the characteristics of modern flow regimes can be inferred for ice shelves (e.g. Fahnestock et al., 2000; Glasser and Scambos, 2008), ice streams (e.g. Price and Whillans, 2001; Campbell et al., 2008) and smaller outlet glaciers (e.g. Glasser and Gudmundsson, 2012). Therefore, I firstly assess the modern dynamics of Skelton Glacier that are evident from observational data.

The surface of Skelton Glacier transitions from the elevated ( $\sim 2250 \mathrm{~m}$ asl), low-gradient ice sheet plateau, via a steeper Transantarctic Mountains section, to the near-flat floating portion downstream of the grounding-line. Integrated and interpolated radar-derived bed data (BEDMAP2; Fretwell et al., 2013) reveal a wide basin in the centre of the catchment that is surrounded by high relief (Figure 4.2). The bed slopes gently through the catchment from $\sim 1500$ to $-50 \mathrm{~m}$ asl, but elongate overdeepenings are apparent in topographicallyconfined areas, specifically near The Portal and in Skelton Inlet. At these locations thickening of the glacier occurs. Ice surface velocities (Rignot et al., 2011) highlight the two main flow paths of Skelton Glacier, the south-west trunk and the north-east arm. Each flow path accelerates in the lower reaches, peaking just upstream of the grounding-line (Figure 4.2).

In order to better constrain flow in the catchment, glacier surface flow-stripes were mapped from the satellite-derived Landsat Image Mosaic of Antarctica (LIMA) dataset. The glacier catchment boundary was delimited using the locations and directions of surface flow-stripes, BEDMAP2 surface slope and ice velocity data. This analysis confirms that ice is sourced from the plateau upstream of Skelton Névé. Flow-stripes develop parallel to flow unit borders, forming from lateral compression at shear margins and where rapid basal sliding occurs across rough glacier beds (Glasser and Gudmundsson, 2012), and 
therefore their distribution can help infer stress regimes within the catchment. The main control on the development of flow-stripes in the Skelton Glacier catchment is lateral compression, as shown on the surface of tributary glaciers to the north-east and towards the confluence of flow paths near the grounding-line. However, basal sliding may also occur along the main flow path, as indicated by the initiation of flow-stripes in the centre of Skelton Névé (Figure 4.2).

The relationships between surface structures and the flow properties of the glacier can be better illustrated in a longitudinal profile along the main trunk (Figure 4.3). High resolution CReSIS airborne radar data reveal larger amplitude bed undulations than what is evident in BEDMAP2 data (Fretwell et al., 2013). The bed is characterised by an extensive basin in the upper reaches of the glacier (at $\sim 0-40 \mathrm{~km}$ ), where it drains Taylor Dome before flowing through The Portal and into Skelton Névé. Further overdeepenings occur at $\sim 100-110 \mathrm{~km}$ down-glacier and immediately downstream of the grounding-line, which are separated by a bedrock ridge. Here, the ice thins from almost $1200 \mathrm{~m}$ to $<600 \mathrm{~m}$ before thickening again to nearly $1500 \mathrm{~m}$. InSAR data (Rignot et al., 2011) reveal a nearexponential increase in surface velocity towards the bedrock ridge and grounding-line, which is complemented by similar TerraSAR-X values and in situ GPS measurements (Golledge et al., 2014a).

The dynamic response of an outlet glacier to perturbations is sensitive to along-flow variations in both bed topography and valley width (Jamieson et al., 2012; Enderlin et al., 2013). Therefore, I investigated the distribution of along-flow stresses with respect to valley geometry down the flowline. Once ice enters the névé, the steepening ice surface enables a transition in the flow regime. This is apparent as a stepped increase in surface velocity near the top (at $\sim 60 \mathrm{~km}$ ) and then in the centre (at $\sim 80 \mathrm{~km}$ ) of the névé. This regime shift is also evident from the presence and structure of crevasses, which form perpendicular to the principle strain rate (Price and Whillans, 2001; Glasser and Scambos, 2008). In the upper-mid névé, occurrences of transverse crevasses signify areas of extending flow as the ice velocity increases (Figure 4.3).

Flow in the lower reaches of Skelton Glacier is likely controlled by reductions in valley width, particularly in the basin between Halfway Nunatak and Clinker Bluff. Surface velocity increases as the flow path narrows and basin deepens downstream of $\sim 95 \mathrm{~km}$ (Figure 4.3). Compressive flow dominates towards the narrowest point of the catchment, evident from marginal crevasses. Ice is then forced to thin and steepen over the bedrock ridge, accelerating to $\sim 270 \mathrm{~m} \mathrm{a}^{-1}$ (Rignot et al., 2011), which is manifested as an icefall where crevassing becomes chaotic. Here, surface geometry adjusts as a consequence of bed topography. 

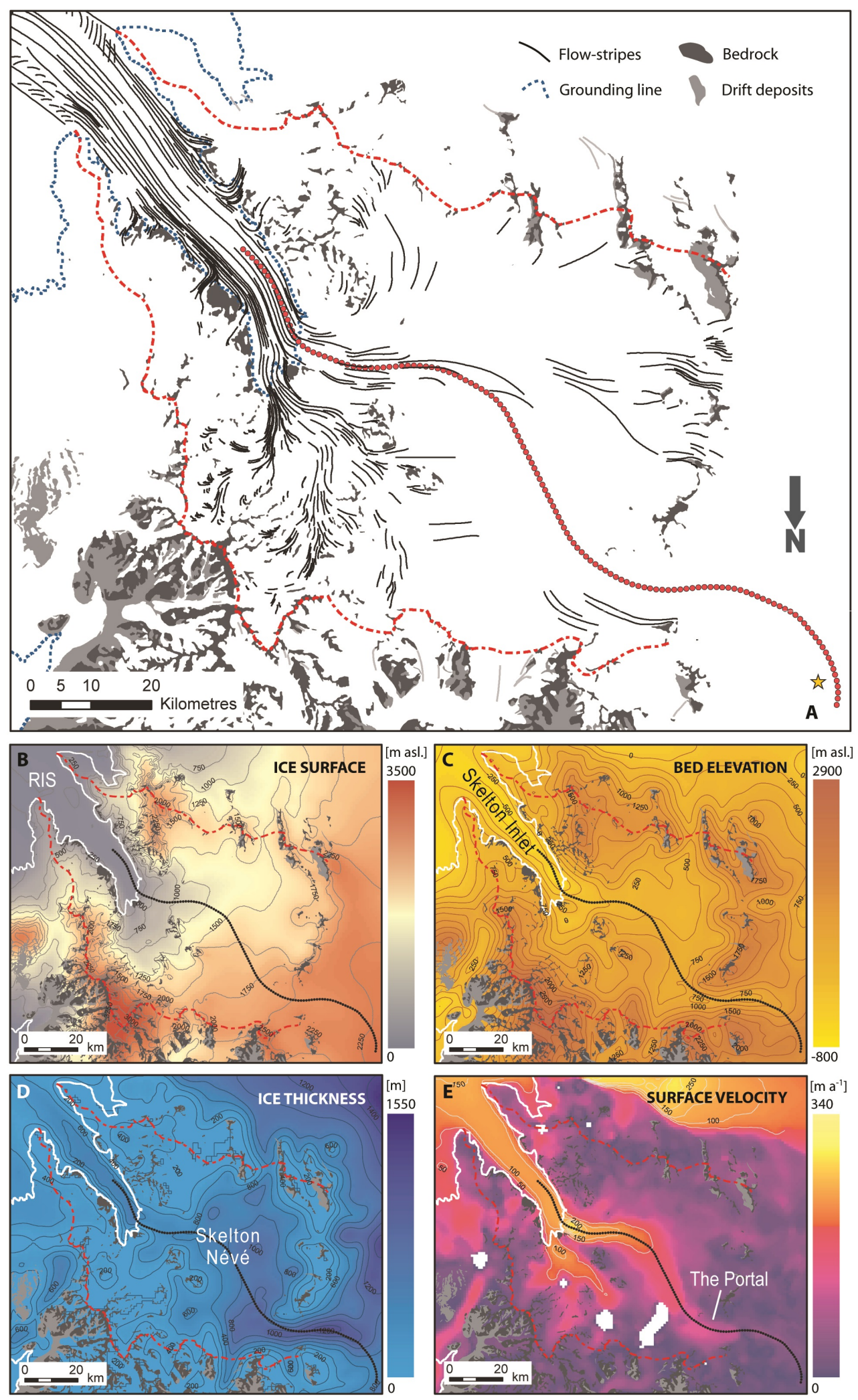

Figure 4.2 Features and characteristics of Skelton Glacier from observational datasets.

A) Flow-stripes, mapped using LIMA imagery, highlight flow paths within the catchment (dashed red line). A glacier flowline (1-km resolution, red circles) extends

from Taylor Dome, near to the ice core site (yellow star), to downstream of the present-day grounding-line. B-E) Ice surface elevation, bed elevation and corresponding ice thickness (BEDMAP2; Fretwell et al., 2013), as well as surface velocity magnitude of Skelton Glacier (Rignot et al., 2011). 

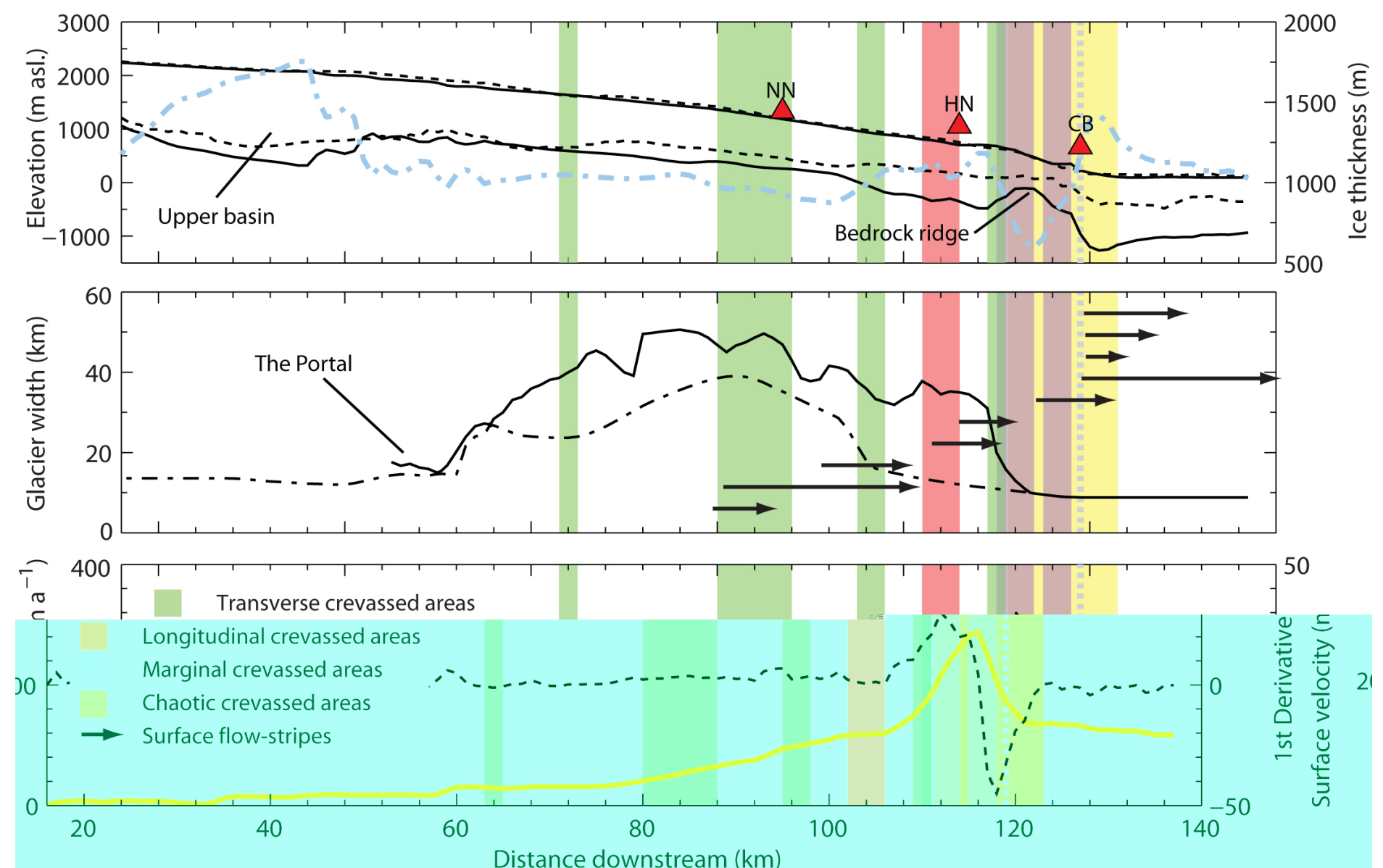

Figure 4.3 Flow of Skelton Glacier from observations along the central flowline. Upper panel: Measured ice surface, bed topography and ice thickness. Bed estimates from CReSIS (solid black line), with corresponding ice thickness (dot-dashed blue line), reveal large overdeepenings not apparent from BEDMAP2 data (dashed black line). Locations of adjacent nunataks (Névé Nunatak, NN; Halfway Nunatak, HN; Clinker Bluff, CB) are denoted as triangles. Middle panel: Width of glacier along the flowline, to and from the catchment boundaries (solid line) and margins of the flow path (dot-dashed line), which shows widening into Skelton Névé downstream of The Portal and narrowing towards Skelton Inlet. Lower panel: Ice surface velocity down-glacier (orange line) and its 1st derivative (dashed black line) (after Rignot et al., 2011), highlight glacier acceleration towards the grounding-line (dashed grey line at $119 \mathrm{~km}$ ). 
Preliminary assessment of Skelton Glacier from observational data suggests several important flow characteristics: 1) Flow extends from Taylor Dome on the Antarctic plateau, providing flux beyond just Skelton Névé and small tributaries from the north-east; 2) The main trunk flows towards the centre of Skelton Névé and adjacent to the line of nunataks, accelerating in its lower reaches; 3) Extending flow and increased velocity likely occurs as a result of a steepening surface, possibly with a basal sliding component, which then changes to compressive flow as the glacier narrows with a dynamic adjustment of ice flux over the bedrock ridge. Under these interpreted present-day conditions, flow by internal deformation can be inferred along most of Skelton Glacier, with higher driving stress, basal shear and the possibility of basal sliding occurring in the lower reaches of the glacier, upstream of the grounding-line.

\subsection{Glacier sensitivity to environmental and physical controls}

In order to further investigate the along-flow dynamics of Skelton Glacier, numerical flowline modelling was carried out. In addition to simulating the glacier under modern conditions, numerical modelling enables the investigation of dynamics for different boundary condition scenarios (e.g. Huybrechts and Oerlemans, 1990; Jamieson et al., 2012; Winkelmann et al., 2012; Briggs et al., 2013; Davies et al., 2014; Maris et al., 2014). I use a 1-d flowline model to predict the likely basal ice temperatures, velocity fields and along-flow extent of basal sliding and internal deformation of Skelton Glacier. The dynamic sensitivity of the glacier is investigated with respect to changes in flow parameterisation, as well as environmental forcings.

\subsubsection{Model set-up and empirical constraints}

The 1-d finite difference flowline model used here has been previously applied to Transantarctic Mountain outlet glaciers (Chapter 3; Golledge and Levy, 2011; Golledge et al., 2014a). Ice thickness evolves in space and time following the mass conservation equation, and was applied here at a 1-km horizontal resolution from the ice divide at Taylor Dome to just downstream of the present-day grounding-line. Flow of the fully grounded portion of Skelton Glacier was calculated using the shallow ice approximation and a longitudinal averaging scheme (Chapter 3). In Skelton Inlet where ice is only loosely grounded, if at all (Crary, 1966), mass loss occurs wherever the bed is below sea level; sub-ice melt or calving is calculated at each cell downstream of the grounding-line, dependent on the ice 
thickness relative to floatation thickness and water depth (see Chapter 3).

The model was initialised with airborne-derived bed topography and ice thickness data (CReSIS, $\pm 10 \mathrm{~m}$ ), extending $136 \mathrm{~km}$ along the main flow path of Skelton Glacier (Figure 4.2). A glacier width was prescribed that varies down-glacier with the margins of the flow path; Skelton Glacier flows through The Portal, widens in Skelton Névé and then narrows upstream of the bedrock ridge in the lower reaches (Figure 4.3).

Simulation of the along-flow dynamics of Skelton Glacier firstly requires the present-day profile to be replicated as closely as possible. This was achieved by tuning uncertain flow and environmental parameters while maintaining physical constants (Table 4.1) and measured climate variables (Table 4.2). Empirical data suggest a complex, non-linear precipitation pattern within the catchment, and initial assessment of Skelton Glacier revealed an additional, minor influx of ice from outside the main flow path modelled here. This can be accounted for by adjusting both the amount of precipitation at sea level and the precipitation lapse rate.

Table 4.1 Physical constants and model parameters

\begin{tabular}{lcc}
\hline Parameter & Value & Unit \\
\hline Flow enhancement factor $*$ & 2 & - \\
Sliding rate factor $*$ & $5 \times 10^{-7}$ & $\mathrm{~Pa} \mathrm{~m}^{2} \mathrm{a}^{-1}$ \\
Glen's flow law exponent & 3 & - \\
Sliding exponent & 2 & - \\
& & \\
Geothermal heat flux & $56-61$ & $\mathrm{~mW} \mathrm{~m}^{-2}$ \\
Thermal conductivity of ice & 2.4 & $\mathrm{~W} \mathrm{~m}^{-1} \mathrm{~K}^{-1}$ \\
Universal gas constant & 8.314 & $\mathrm{~J} \mathrm{~mol}^{-1} \mathrm{~K}^{-1}$ \\
Horizontal domain resolution & 1000 & $\mathrm{~m}$ \\
\hline
\end{tabular}

*Tuning parameter

\subsubsection{Simulating present-day Skelton Glacier}

The tuned Skelton Glacier simulation largely reproduces the observed surface profile and ice geometry (Figure 4.4). In particular, the low gradient upper reaches, steepening middle reaches and steep ice-fall at the bedrock ridge are well replicated. The simulated ice thickness co-varies with measurements down-glacier, diverging by $<100 \mathrm{~m}$. These slight mismatches of ice geometry could not be resolved by exploration of tuning parameters, and instead suggest simplifications in the physics of the model and subtle, unaccounted for 
Table 4.2 Environmental parameters (modern)

\begin{tabular}{lcc}
\hline Parameter & Value & Unit \\
\hline Precipitation at sea level $*$ & 1 & $\mathrm{~m} \mathrm{a}^{-1}$ \\
Precipitation lapse rate $*$ & -0.02 & $\mathrm{~m} \mathrm{a}^{-1} \mathrm{~km}^{-1}$ \\
Mean annual temp. at sea level $\dagger$ & -21 & ${ }^{\circ} \mathrm{C}$ \\
Temperature lapse rate $\dagger$ & -9 & $\mathrm{~K} \mathrm{~km}^{-1}$ \\
Annual temperature range \# & 25 & ${ }^{\circ} \mathrm{C}$ \\
Ocean temperature (winter) & -1.8 & ${ }^{\circ} \mathrm{C}$ \\
Ocean temperature (summer) $*$ & -1.6 & ${ }^{\circ} \mathrm{C}$ \\
Calving rate coefficient $*$ & 1 & - \\
Relative sea level & 0 & $\mathrm{~m}_{\text {Degree-day factor }}$ \\
\hline
\end{tabular}

*Tuning parameter, $\dagger($ Crary, 1966), \#(Golledge and Levy, 2011).

variations in the glacier width. Despite minor disagreement regarding the magnitude of along-flow profile fluctuations, the simulated Skelton Glacier reproduces observed stepchanges in surface velocity downstream; ice velocity accelerates slightly from $60 \mathrm{~km}$, further increases at $80-100 \mathrm{~km}$ and $110 \mathrm{~km}$, and peaks at $116 \mathrm{~km}$ (Rignot et al., 2011) (Figure 4.3).

The first objective of this work was to identify present-day controls on the flow of Skelton Glacier. The preliminary investigation recognised predominantly topographicallyconstrained, surface slope-driven flow by ice deformation, with the possibility of ice thickness-controlled sliding in the lower reaches of the glacier. The modelled Skelton Glacier supports this initial assessment, with simulated flow by deformation greater than by sliding along the length of the glacier and enhanced sliding simulated in its lower reaches. Areas of basal sliding correspond with the locations of thicker ice in the overdeepened basins, where basal melt is predicted. In its current configuration, dynamic ice occurs in the laterally-constrained lower reaches of Skelton Glacier, with most vigorous flow occurring where thinner ice and steeper surface slopes are found. Based on these observations, I hypothesise that the lower reaches of the glacier and possibly ice in the overdeepened basins are most sensitive to environmental perturbations.

\subsubsection{Sensitivity of Skelton Glacier to environmental variables}

The second objective of this study was to investigate the response of Skelton Glacier to changes in environmental variables. The sensitivity of the glacier was tested for climatic perturbations that reflect variance around the simulated present-day values; these include mean annual air temperature (MAAT), precipitation (changed at sea level), precipitation 

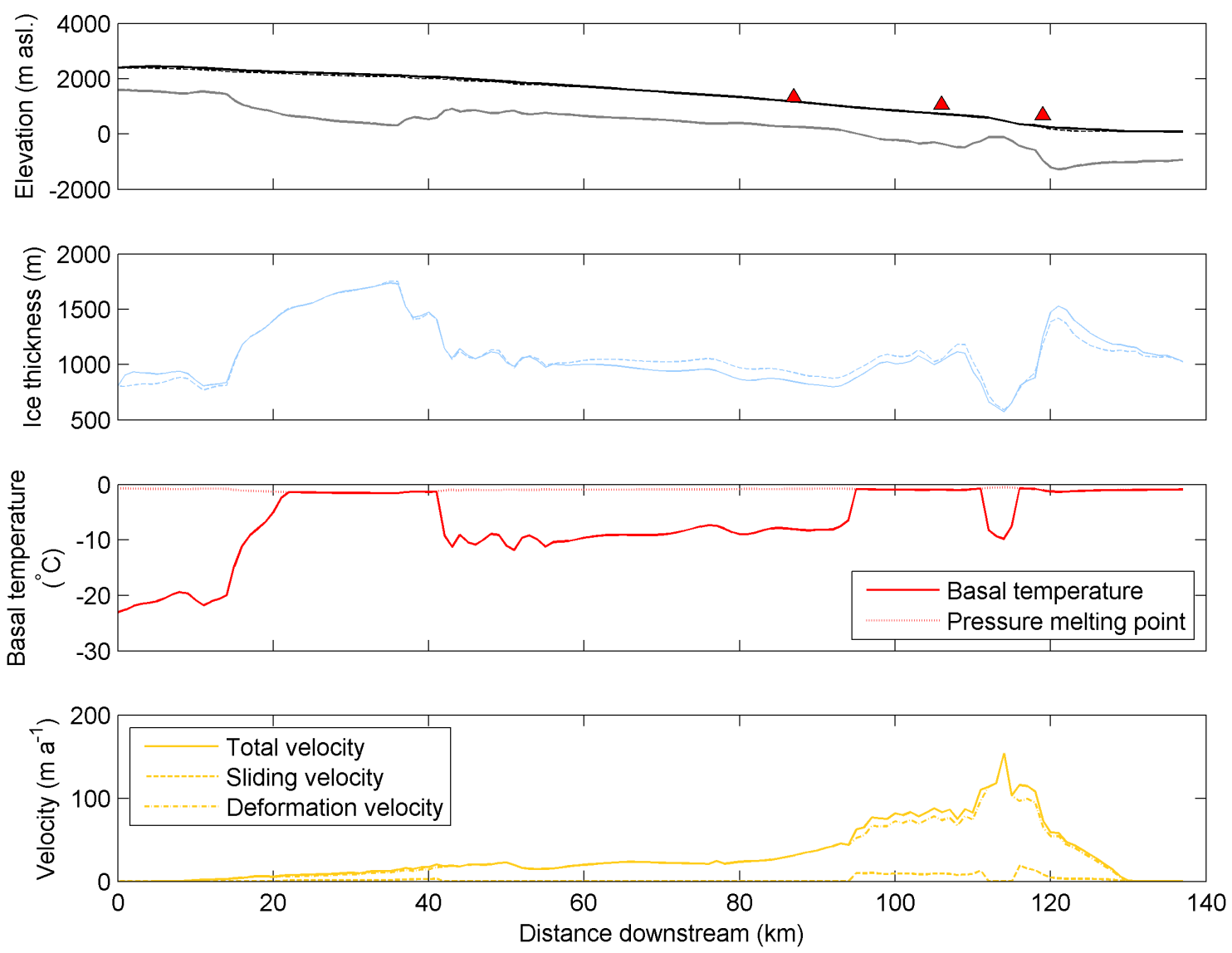

Figure 4.4 Simulated present-day Skelton Glacier. The modelled surface (black, solid) and bed (grey, solid) elevations are consistent with the measured (CReSIS) profiles (dashed), manifested a similar along-flow variations in ice thickness. Flow is predominantly by ice deformation, but sliding is simulated in the overdeepened basins where thick ice enables the basal temperature to reach the pressure melting point.

lapse rate and summer ocean temperature (with winter ocean temperature kept at $-1.8^{\circ} \mathrm{C}$ ) (Table 4.3). Similarly, the effect of relative sea level change was also investigated by simulating Skelton Glacier for a range sea level values relative to present-day (of $-30 \mathrm{~m}$ asl to +50 $\mathrm{m}$ asl, in $5 \mathrm{~m}$ increments). The geometry, and therefore dynamics, of Transantarctic Mountain outlet glaciers is significantly influenced in their lower reaches by the presence of grounded ice in the Ross Sea, which provides a buttressing effect that is independent of local environmental variables. However, the extent of Antarctic ice sheets and hence presence or absence of this buttressing effect downstream of Skelton Glacier is dependent on global climate conditions. Therefore we additionally explore this thickening in the lower reaches of the glacier by changing the calving coefficient (from 0.2 to 1 , in 0.1 increments), which acts to limit mass loss at the terminal cell thereby representing the effect of buttressing.

A first measure of the steady-state response to changes in environmental parameters is ice volume, represented here as a 2-d volume simulated for the flowline catchment. A second 
Table 4.3 Climate parameters used to investigate the sensitivity of Skelton Glacier

\begin{tabular}{lcccc}
\hline Parameter & $\begin{array}{c}\text { Range } \\
\text { (value) }\end{array}$ & $\begin{array}{c}\text { Range } \\
\text { (\%) }\end{array}$ & $\begin{array}{c}\text { Increments } \\
\text { (value) }\end{array}$ & Unit \\
\hline Precipitation at sea level & 0.4 to 1.2 & -60 to +20 & 0.05 & $\mathrm{~m} \mathrm{a}^{-1}$ \\
Precipitation lapse rate & 0.1 to 0.24 & -50 to +20 & 0.01 & $\mathrm{~m} \mathrm{a}^{-1} \mathrm{~km}^{-1}$ \\
Mean annual temp. at sea level & -33 to -11 & -60 to +50 & 1 & ${ }^{\circ} \mathrm{C}$ \\
Ocean temperature (summer) & -1.8 to -1 & -12 to +40 & 0.1 & ${ }^{\circ} \mathrm{C}$ \\
\hline
\end{tabular}

measure of steady-state glacier change is ice surface elevation. Here, I use the elevation at the grounding-line as it provides the most consistent trend (Figure 4.5); when applying a linear negative mass balance change, the elevation at and near the terminal cell records a highly variable and positive response, possibly a model artefact arising from surface readjustment at the end of the domain. Furthermore, changes in surface elevation, and therefore likely ice thickness, at the grounding-line have greater implications for outlet glacier mass loss (Rignot and Thomas, 2002) and stability (Schoof, 2007).

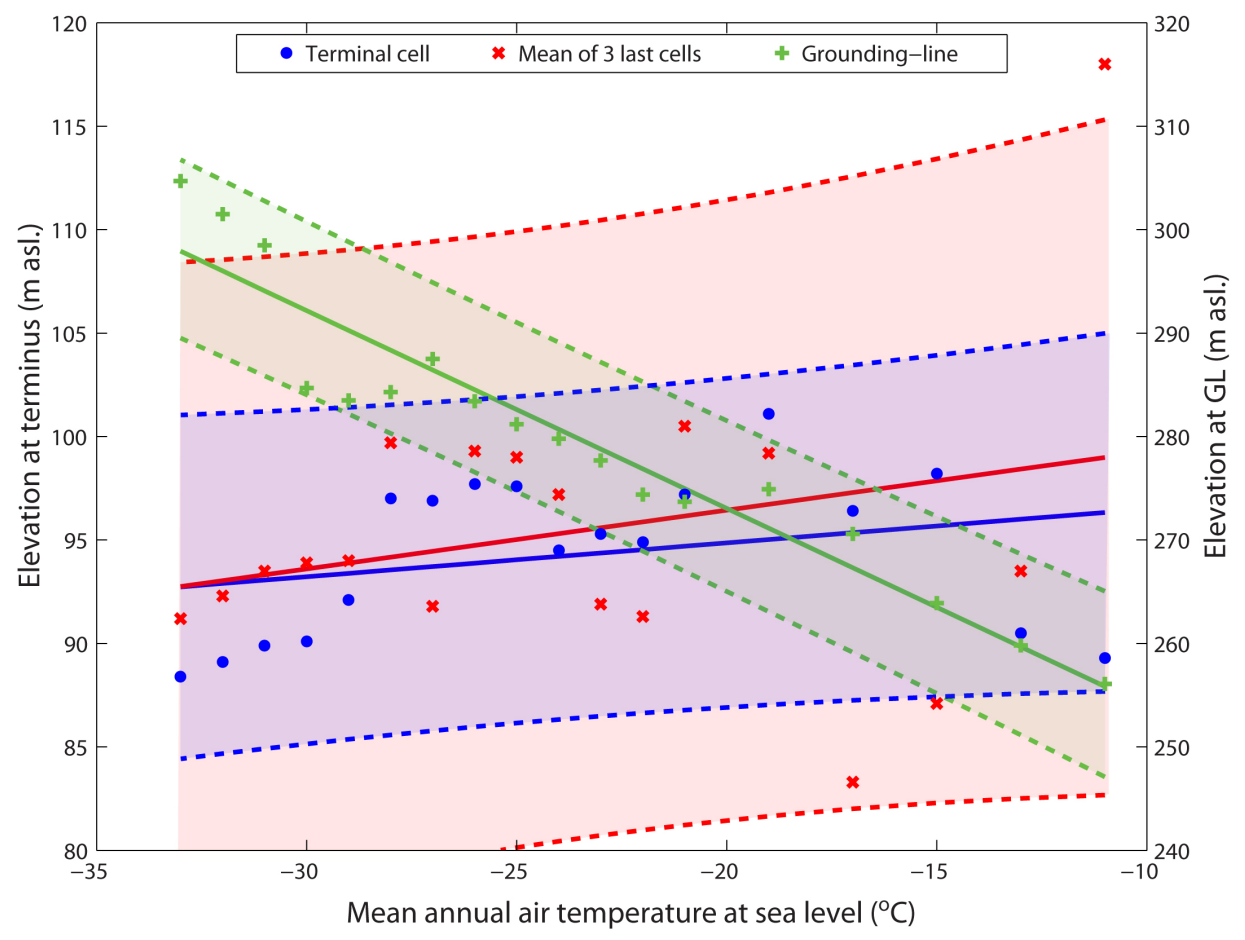

Figure 4.5 Test of surface elevation as a measure for changes in steady-state glacier profile. Surface elevations are shown for the terminal cell, the mean of the three terminal cells and the grounding-line (GL), that result from a linear change in air temperature.

Bold lines show the respective linear regressions, while the dashed lines denote the 2-sigma standard deviation. A highly variable surface elevation is simulated at the terminal cell and of the final three cells, and despite a warming temperature trend, a slight increase in elevation of the surface occurs. Elevations at the grounding-line have a stronger, negative trend and will be used as a measure in sensitivity experiments as they better reflect changes in steady-state geometry. 
The largest changes in glacier geometry occur as a result of the externally forced thickening of the lower reaches, achieved here through a reduced calving coefficient (Figure 4.6). A small reduction in calving produces a negligible ice volume change and a minor increase in the surface elevation. However, once ice thickens at the grounding-line so that the surface elevates above $\sim 500 \mathrm{~m}$ asl (calving coefficient 0.7 ), both the surface elevation and ice volume increase rapidly in response to step decreases in calving. This relationship likely results from decreased driving stress from an increasingly shallow surface slope, and therefore reduced flux and mass loss at the terminus, thickening in the lower reaches of the glacier and further decreases in driving stress, modulated by mass loss via calving.

Similar non-linear relationships are simulated for changes in ocean temperature and sea level (Figure 4.6). For an increase in ocean temperature above present, both the surface elevation at the grounding-line and ice volume change exponentially. A change in sea level below present produces an increase in glacier volume, whereas a sea level above present results in a minor decrease in ice volume. The ice surface at the groundingline demonstrates an opposite relationship, whereby the surface elevates from a rise in sea level, possibly as thicker ice can be facilitated at the grounding-line, despite greater volume loss through calving. However, these changes from sea level perturbations do not significantly affect the glacier geometry, where at most, $\sim 10 \mathrm{~km}^{3}$ volume and $<10 \mathrm{~m}$ elevation change occur per $20 \mathrm{~m}$ sea level change.

The geometry of Skelton Glacier responds linearly to a step change in MAAT, precipitation and precipitation lapse rate (Figure 4.6). An increase in both precipitation and precipitation lapse rate increases the ice volume and the ice surface at the grounding-line, whereas a negative relationship exists between MAAT and the glacier geometry (Figure 4.6). The magnitude of these effects also differs between the environmental parameters. A $\sim 20 \%$ atmospheric (MAAT) warming (from $-21^{\circ} \mathrm{C}$ to $-17^{\circ} \mathrm{C}$ ) produces $\sim 170 \mathrm{~km}^{3}(\sim 8 \%$ ) volume loss. However, an equivalent $20 \%$ change in precipitation and precipitation lapse rate only results in a $\sim 4 \%\left(\sim 80 \mathrm{~km}^{3}\right)$ and $\sim 1 \%\left(\sim 25 \mathrm{~km}^{3}\right)$ ice volume change, respectively. While in reality any changes in regional precipitation are likely coupled to changes in atmospheric temperature, these sensitivity tests at Skelton Glacier highlight that air temperature is the likely dominating factor influencing glacier geometry.

Ice flowing through the overdeepened basins and in the lower reaches of Skelton Glacier are hypothesised to be most sensitive to environmental perturbations. Therefore, I explore the along-flow dynamic sensitivity to changes in calving, precipitation and atmospheric temperature. Incrementally reducing the calving coefficient results in a progressively thicker glacier in its lower reaches with diminishing thickening upstream, replicating the effect of buttressing at the terminus. Today, basal temperatures reach the pressure melting 

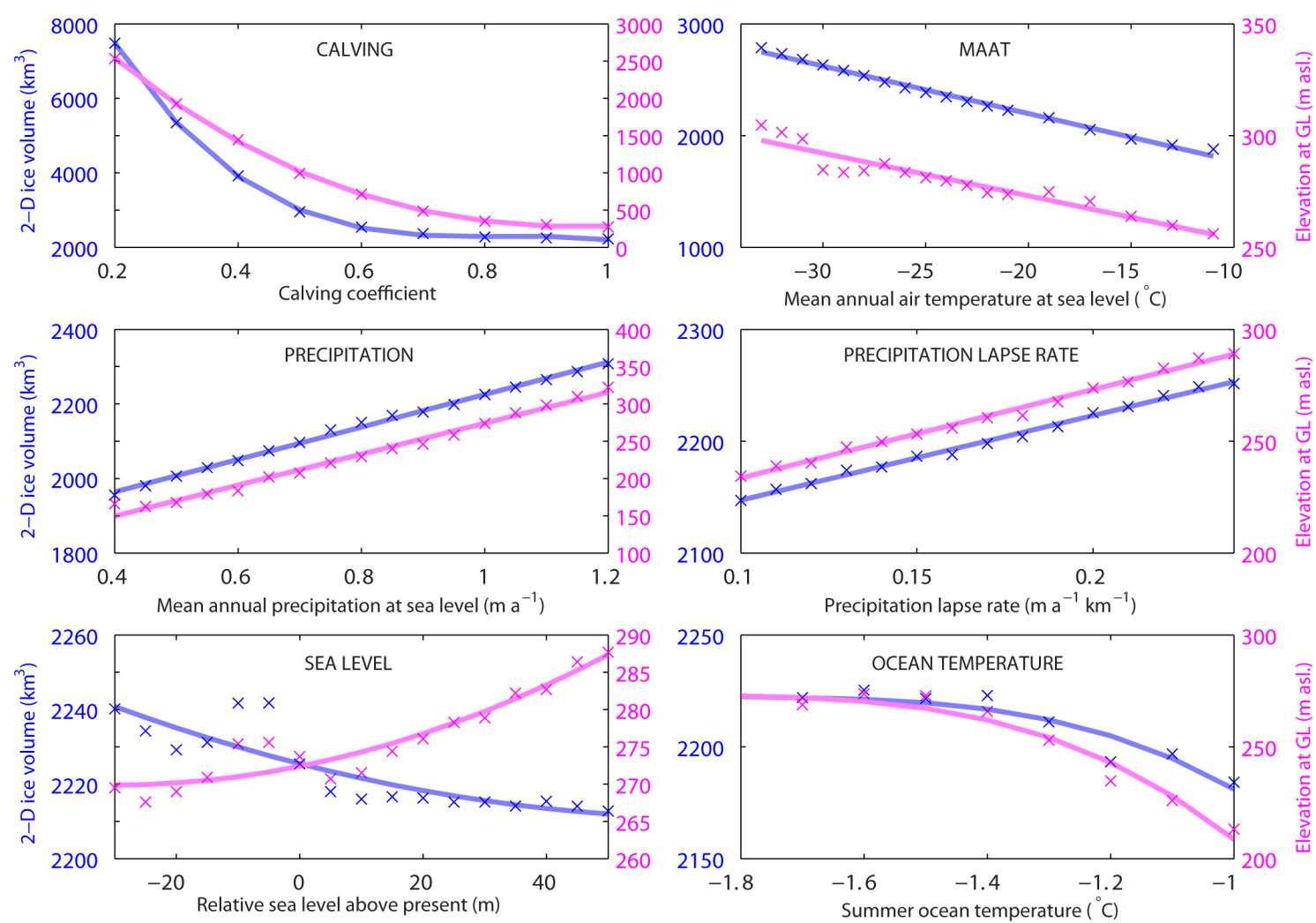

Figure 4.6 Sensitivity of Skelton Glacier to environmental variables. Ice volume and elevation at the grounding-line (GL) have linear relationships with perturbations in mean annual air temperature (MAAT), amount of precipitation and precipitation lapse rate, but has a greater response for MAAT per percentage change. Calving, sea level and ocean temperature produce non-linear geometric responses, and is exponential for the latter of these.

point in the upper basin and in the smaller overdeepenings either side of the bedrock ridge. Under a buttressed Skelton Glacier scenario, thickening extends the area of the bed that is at pressure melting point, suggesting that basal melt could occur in the portion upstream of Névé Nunatak. The glacier is forced to thin over the bedrock ridge that currently prohibits basal melt, however, melt can be enabled here with sufficient thickening; an increase of ice thickness by $\sim 170 \mathrm{~m}$ (calving coefficient of 0.8 to 0.7 ) exceeds this threshold, causing the basal ice temperature to reach pressure melting point.

While changes in both precipitation and atmospheric temperature produced linear ice geometry responses, the dissimilar magnitudes of ice volume change suggest differing dynamic effects along the length of Skelton Glacier. An increase in precipitation causes a slight thickening along the profile, with an opposite effect under reduced precipitation. While the area of basal ice that reaches melting point is unaffected by these changes, the minor thickening from increased accumulation acts to increase basal temperature, dominating over possible cooling effects from enhanced precipitation. This slight warming of the glacier acts to soften the ice and in turn, together with strain heating, is able to facilitate greater deformation velocities in the steeper lower reaches of the glacier. 

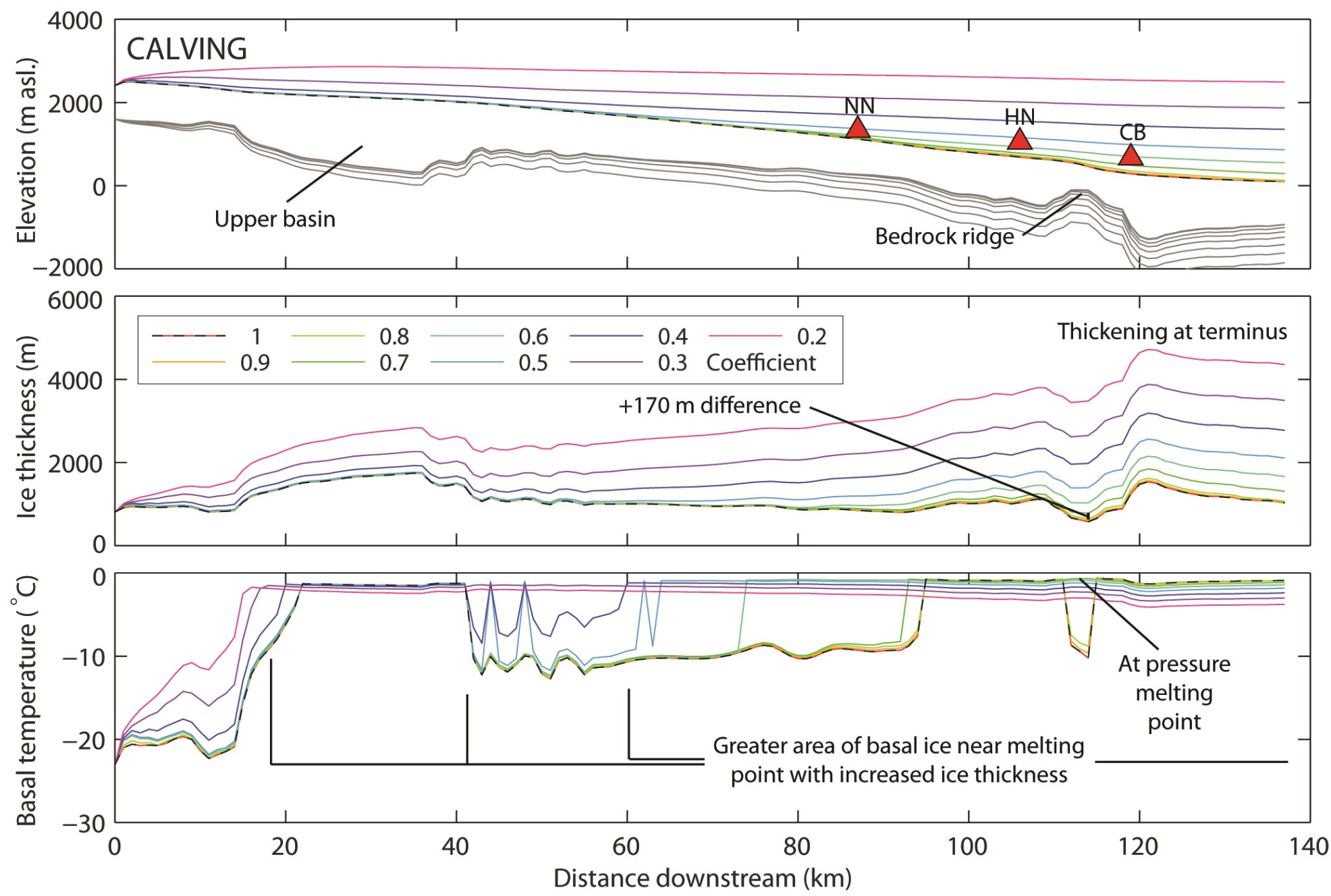

Figure 4.7 Simulated along-flow sensitivity of Skelton Glacier to changes in calving. Similar to a buttressing effect, changes in the calving coefficient cause adjustment of ice thickness in the lower reaches. Under thicker ice, the basal ice temperature increases, and at the bedrock ridge, a $\sim 170 \mathrm{~m}$ thickening enables a likely switch to basal melt. Present-day profiles are shown by a dashed line, while nunataks are red triangles (labelled as in Figure 

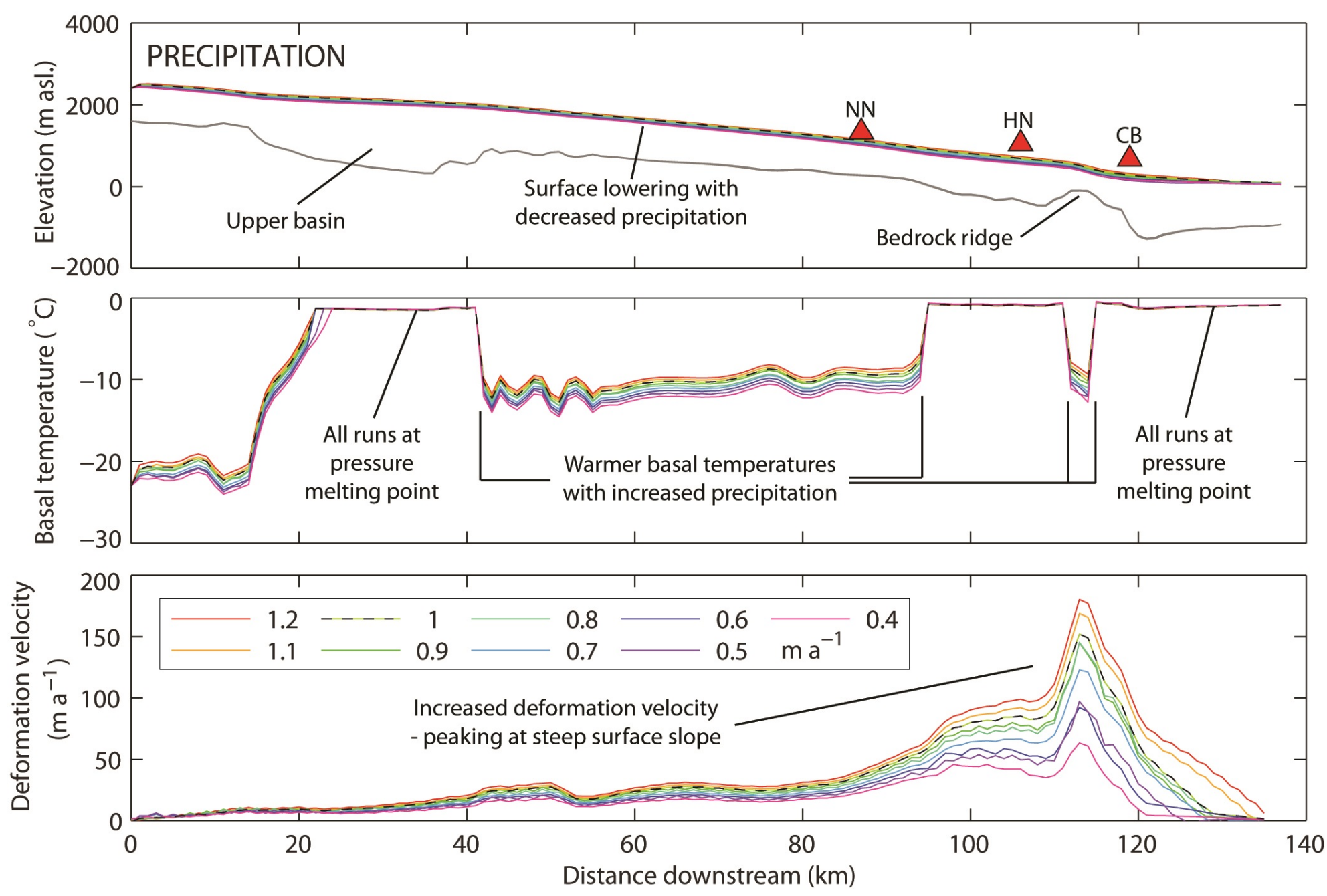

Figure 4.8 Simulated along-flow sensitivity of Skelton Glacier to changes in precipitation. Minor changes are predicted in the surface elevation and extent of basal melt. However, the slight thickening from increased precipitation acts to warm ice at the bed, and internal feedbacks allow large amplitude ice deformation changes in the lower reaches. Present-day profiles are shown by a dashed line, while nunataks are red triangles (labelled as in Figure 4.3). 

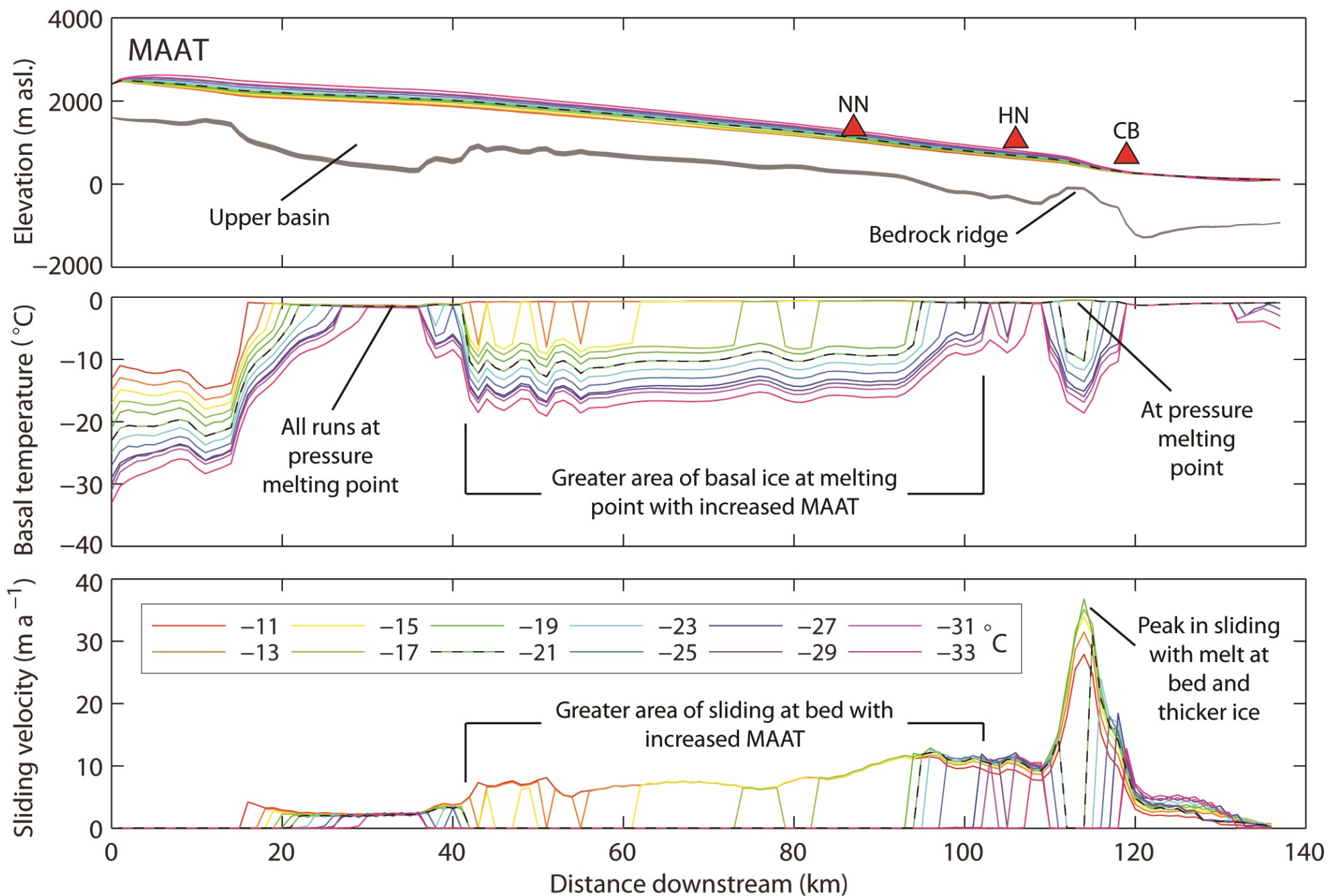

Figure 4.9 Simulated along-flow sensitivity of Skelton Glacier to changes in mean annual air temperature (MAAT). Despite small surface elevation effects, changes in MAAT are able to alter the area of basal ice reaching melting point, with $-19^{\circ} \mathrm{C}$ causing basal melt to occur at the bedrock ridge. Melt at the bed enables a greater extent and magnitude of basal sliding, which is limited by ice thickness in the lower reaches. Present-day profiles are shown by a dashed line, while nunataks are red triangles (labelled as in Figure 4.3). 
A contrasting dynamic response is simulated for changes in air temperature, despite similar low-magnitude ice thickness adjustments. Warmer air temperatures lead to a negative surface mass balance, causing the glacier to thin, however, they also substantially increase the extent of basal melt along the profile. In particular, a $2^{\circ} \mathrm{C}$ increase in mean annual air temperature from $-21^{\circ} \mathrm{C}$ (present) to $-19^{\circ} \mathrm{C}$ enables basal melt at the bedrock ridge. The $2{ }^{\circ} \mathrm{C}$ rise above present at the bedrock ridge allows the glacier to switch from zero sliding here to peak sliding along the profile that can be explained by a positive feedback; as ice deformation rises from warmer surface temperatures, strain heating on slopes is able to increase, and then sliding increases once basal melt is possible, which encourages more strain heating. Sliding at the bed thins the glacier along its length and this reduced ice thickness then hinders the sliding potential, which is shown at the bedrock ridge for warmer glacier scenarios that result from air temperatures $>-19^{\circ} \mathrm{C}$. The occurrence of melt along more of the bed allows for both more extensive and higher magnitude sliding at the bed. Sliding would likely extend continuously from $\sim 25 \mathrm{~km}$ upstream of Névé Nunatak to the terminus under a $6^{\circ} \mathrm{C}$ warmer air temperature, and from the head of the upper basin under $8-10^{\circ} \mathrm{C}$ warmer temperatures.

In summary, the volume and surface elevation of Skelton Glacier can respond linearly or exponentially, depending on which environmental variable is changed. In particular, buttressing at the terminus from expanded ice in the Ross Sea allows significant thickening of the lower reaches and therefore a greater glacier volume. Thickening has the potential to increase the area of basal melt along the glacier, however, the basal conditions are also dependent on the environmental conditions at the surface. For example, changes in precipitation can affect the temperature of the ice that then influences the amount of ice deformation, however, this causes only moderate volume change. Variations in mean annual air temperature lead to greater adjustments in ice volume than from precipitation, primarily from its ability to modify the extent of basal melt and therefore sliding at the bed. These sensitivity tests also identify that areas of the bed can switch between contrasting dynamic states if near an ice thickness threshold, for example, at the bedrock ridge.

\subsection{Dynamics and basal conditions of Skelton Glacier under different climates}

The third objective of this work was to investigate the dynamics and basal conditions of Skelton Glacier under past climates. Sensitivity experiments illustrate that the glacier responds differently along its length depending on the environmental conditions. Here, we investigate variations in the dynamics for contrasting LGM (colder and drier), Pliocene 
interglacial (warmer and wetter) and Pliocene glacial (colder and wetter) regimes.

Steady-state simulations are constrained by regional empirical evidence of past climatic conditions and ice sheet configurations (Table 4.4). During the LGM, accumulation was minimal $\left(\leq 0.01 \mathrm{~m} \mathrm{a}^{-1}\right)$ and atmospheric temperatures were $\sim 9-10^{\circ} \mathrm{C}$ colder than present at Taylor Dome (Steig et al., 1998; Monnin et al., 2004). The glacier was likely thicker in its lower reaches as geological data indicates ice surface elevations of 800 and $640 \mathrm{~m}$ asl south and north of the mouth of Skelton Glacier (Bockheim et al., 1989; Anderson et al., 2004; Denton and Marchant, 2000). A relative sea level of $+50 \mathrm{~m}$ is also inferred for this time, based on the output from a global isostatic model (Peltier, 2004). For the Pliocene, interglacial conditions are prescribed in the model based on inferences of a globally warmer and wetter climate (Lisiecki and Raymo, 2005; Fedorov et al., 2013; Haywood et al., 2013) and regional sea surface temperatures as high as $3-5^{\circ} \mathrm{C}$ (Winter et al., 2010). Under these conditions, the Skelton Inlet was likely free of grounded ice as geological evidence and modelling suggests that the Ross Embayment was largely open water (Naish et al., 2009; Pollard and DeConto, 2009), which is also reflected by a eustatic sea level higher than present (+25 m) at this time (Dwyer and Chandler, 2009). Pliocene glacial episodes are poorly constrained, however thickening in the lower reaches of the glacier probably occurred as grounded ice extended past the mouth of Skelton Glacier (Naish et al., 2009; Pollard and DeConto, 2009), and a climate warmer and wetter than the LGM but colder than present can be inferred. All other environmental parameters in these simulations are largely unknown so are kept as modern values, and model flow parameters remain unchanged from the modelled present-day glacier.

Table 4.4 Environmental parameters (past)

\begin{tabular}{lcccc}
\hline Parameter & LGM & $\begin{array}{c}\text { Pliocene } \\
\text { (interglacial) }\end{array}$ & $\begin{array}{c}\text { Pliocene } \\
\text { (glacial) }\end{array}$ & Unit \\
\hline Precipitation at sea level $*$ & 0.01 & 2 & 1 & $\mathrm{~m} \mathrm{a}^{-1}$ \\
Precipitation lapse rate & -0.02 & -0.02 & -0.02 & $\mathrm{~m} \mathrm{a}^{-1} \mathrm{~km}^{-1}$ \\
Mean annual temp. at sea level $*$ & -30 & -10 & -25 & ${ }^{\circ} \mathrm{C}$ \\
Temperature lapse rate & -9 & -9 & -9 & $\mathrm{~K} \mathrm{~km}^{-1}$ \\
Annual temperature range & 25 & 25 & 25 & ${ }^{\circ} \mathrm{C}$ \\
Ocean temperature (winter) & -1.8 & -1.8 & -1.8 & ${ }^{\circ} \mathrm{C}$ \\
Ocean temperature (summer) $*$ & -1.8 & +0.5 & -1.6 & ${ }^{\circ} \mathrm{C}$ \\
Calving rate coefficient $*$ & 0.121 & 1 & 0.6 & - \\
Relative sea level $*$ & +50 & +25 & +40 & $\mathrm{~m}$ \\
\hline
\end{tabular}

*Parameter informed by interpretations of ice core or geological data (see text).

These data-constrained simulations of Skelton Glacier reveal adjustments of the glacial regime in response to changing ice geometry and environmental conditions during con- 


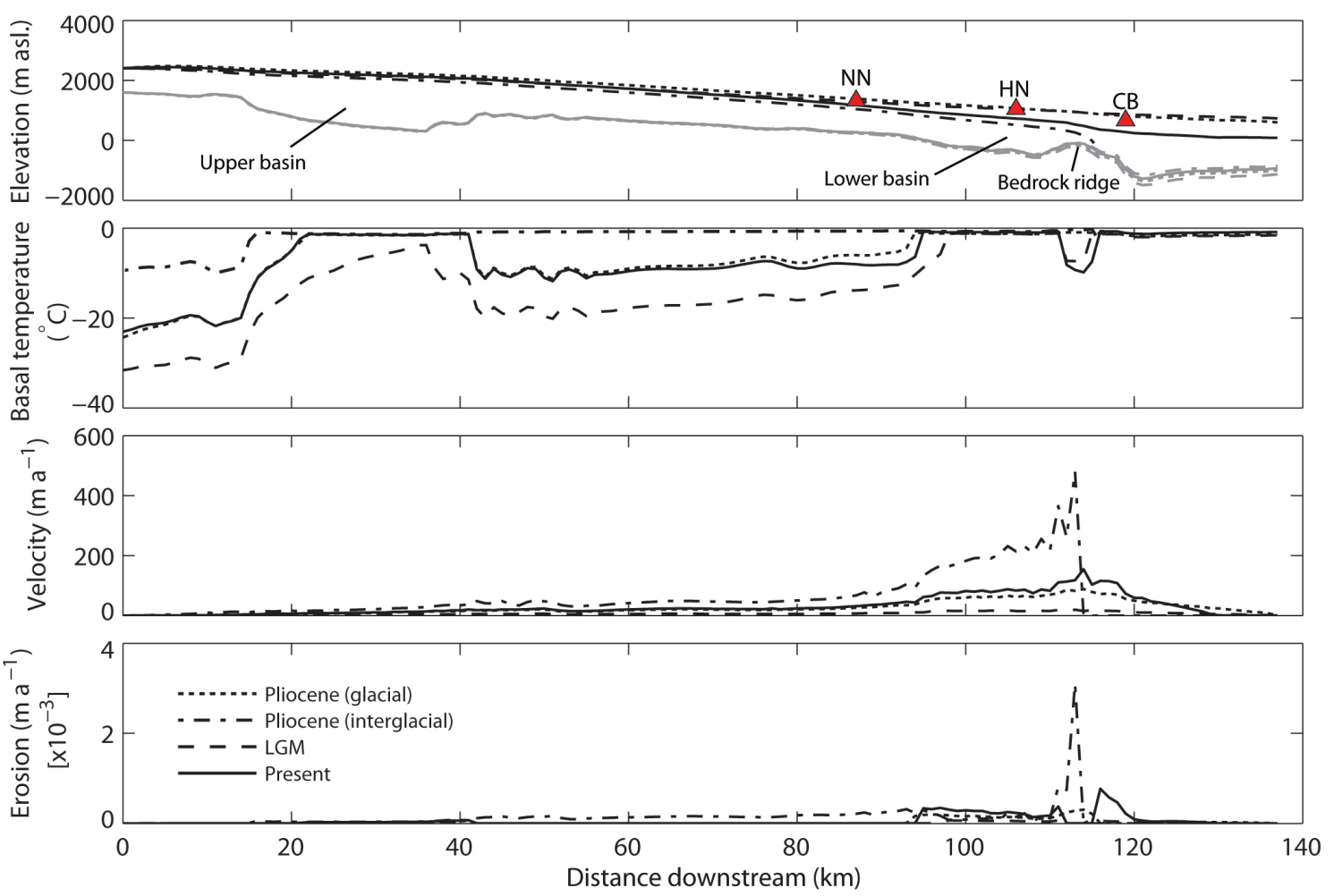

Figure 4.10 Simulated configurations and dynamics of Skelton Glacier under present-day, LGM, Pliocene interglacial and Pliocene glacial climates. The glacier was likely thick enough during glacial (LGM and Pliocene) climates to override the nunataks (red triangles, labelled as in Figure 4.3) and was fully grounded, above sea level, during warm interglacial climates, when near-continuous basal sliding and erosion predicted along the central flowline.

trasting climatic episodes. Today, basal melt likely occurs in the upper and lower basins as well as downstream of the bedrock ridge, while moderate basal erosion $\left(<0.001 \mathrm{~m} \mathrm{a}^{-1}\right)$ is predicted in the overdeepenings of the lower reaches of the glacier where velocities are high. The modelled glacier geometry at the LGM reproduces a low gradient surface profile, with thickening in the lower reaches of Skelton Glacier sufficient to cover the nunataks adjacent to the flow path. This thickening causes slightly warmer than present basal ice at the bedrock ridge, but elsewhere basal temperatures are simulated to be cooler and basal melt in the lower basin (at $\sim 100 \mathrm{~km}$ ) is less extensive than today. Together, the shallow surface slope and small extent of basal melt produce very low velocities, with minor erosion at the bed limited to the overdeepenings either side of the bedrock ridge. During a simulated Pliocene interglacial regime, warm ocean temperatures force the glacier to terminate at the bedrock ridge above sea level, with a lower than present surface profile near the terminus. Despite this reduced ice thickness, warm atmospheric temperatures allow basal melt to occur from the upper basin to the terminus, with nearcontinuous basal erosion predicted along this length of the glacier. A steep ice surface in the lower reaches greatly enhances the velocity, which peaks $\left(>400 \mathrm{~m} \mathrm{a}^{-1}\right)$ together with basal erosion $\left(\sim 0.003 \mathrm{~m} \mathrm{a}^{-1}\right)$ at the terminus. A buttressed Skelton Glacier during 
Pliocene glacial episodes probably had a surface profile similar to that at the LGM, however the relatively warmer and wetter climate at this time instead results in velocities and basal conditions similar to today. One key difference between present-day and Pliocene glacial regimes occurs at the bedrock ridge, where the thicker ice of the Pliocene configuration facilitates basal melt that, in turn, enables modest erosion at the bed $(\sim 0.0005 \mathrm{~m}$ $\left.\mathrm{a}^{-1}\right)$.

\subsection{Discussion}

\subsubsection{Topographically-controlled flow of Skelton Glacier}

Evaluation of the Skelton Glacier system from satellite and aerial observations as well as flowline modelling provides new insight into the controls on the flow of Transantarctic Mountain outlet glaciers. Skelton Glacier thickens upstream of large bed undulations, for example at the bedrock ridge above the grounding-line, which causes the ice surface to steepen down-glacier in a similar manner to neighbouring Ferrar Glacier (Golledge and Levy, 2011). Surface velocity peaks where the surface is steepest, however, the velocity increases further upstream where the glacier narrows. Longitudinal extending flow is coincident with this accelerating surface velocity but not with the presence of basal melt, and therefore indicates that flow is controlled primarily by changes in surface slope and glacier width; mass conservation occurs whereby the ice flux dynamically adjusts to a varying geometry. These results are similar to the along-flow interpretations at Taylor and Ferrar Glaciers (Kavanaugh and Cuffey, 2009; Golledge and Levy, 2011) that ice velocity peaks as it cascades over bed obstacles and is propagated in areas of thicker ice, which is in contrary to the idea of bed topography restricting glacier flow (Johnson and Staiger, 2007). At Skelton Glacier, width is also recognised as an important control on ice flux in the absence of major variations of bed topography. Together, these interpretations have implications for numerous Transantarctic Mountain outlet glaciers, which typically have overdeepened basins in their upper reaches and near their modern grounding-lines, and become more laterally constrained in their downstream reaches (Fretwell et al., 2013).

\subsubsection{Sensitivity of Skelton Glacier to environmental forcings}

Sensitivity experiments revealed that the ice geometry of Skelton Glacier responds linearly or exponentially depending on the environmental forcing. Enhanced oceanic forcing, from a raised relative sea level or ocean temperature, would cause mass loss. This 
effect is simulated to be small for a sea level change above present, but exponential for an increase in ocean temperature above present, indicating a floatation-limiting factor for the modern configuration of Skelton Glacier. Sea level rise results in retreat and mass loss as long as ice thickness is below the floatation threshold (e.g. Jamieson et al., 2014). However, in the overdeepended basin near the grounding-line, loss through floatation is limited whereas increases in ocean temperature can act unimpeded to exponentially increase sub-ice melt (Holland et al., 2008).

Buttressing of Skelton Glacier by either a thicker Ross Ice Shelf or grounded ice in the Ross Sea is demonstrated to likely cause the most significant increases in ice volume. The effect of thickening in the lower reaches acts to reduce the glacier surface slope, thereby reducing driving stress and ice flux, leading to reduced mass loss and potential further thickening. The degree of enhanced lateral drag (or 'buttressing') therefore governs upstream thickening and the potential for further thickening from positive feedbacks. While the transition from grounded to floating ice or the interaction between Skelton Glacier and buttressing ice is not fully incorporated in these modelling experiments, it can be assumed that with sufficient thickening in its lower reaches that exceeds floatation thickness, the grounding-line would advance (Schoof, 2007).

Changes in precipitation and mean annual air temperature lead to linear responses of Skelton Glacier, manifested as adjustments of ice volume and surface elevation at the grounding-line. These relationships are expected for Antarctic glaciers as a decrease in temperature or an increase in precipitation enlarges and elevates the accumulation area. Any changes in surface mass balance that result in increased ice volume, act to thicken ice at the grounding-line with the potential for glacier advance (Seroussi et al., 2014). However, the higher elevation of the glacier surface resulting from increased precipitation can alternatively lead to surface steepening and enhancement of the driving stress and ice flux (Winkelmann et al., 2012). At Skelton Glacier, a positive change in air temperature resulted in a greater modelled ice volume loss than from an equivalent negative percentage change in precipitation. This is a superficially similar to the modelled response of a glacier in the Antarctic Peninsula (at $\sim 64{ }^{\circ} \mathrm{S}$ ), where warming-induced mass loss occurs because of a close proximity to a surface melt threshold, as a result of relatively high local atmospheric temperatures ( $\sim 7^{\circ} \mathrm{C}$ MAAT) (Davies et al., 2014). However, in this case the more southerly location $\left(\sim 78{ }^{\circ} \mathrm{S}\right)$ and therefore significantly colder temperatures $\left(-21^{\circ} \mathrm{C}\right.$ MAAT) at Skelton Glacier, demonstrates that an alternative, ice-dynamic mechanism is required to explain the modelled sensitivity to air temperature.

Outlet glaciers respond dynamically along their length following perturbations in environmental forcings or stresses near the grounding-line (Vieli and Nick, 2011). Bed topography is shown to provide a critical control on the along-flow response at Skelton Glacier, 
principally through its affect on ice thickness and therefore potential basal melt. In particular, threshold responses can result from bed variations in certain locations, for example at the bedrock ridge. Here, a $2^{\circ} \mathrm{C}$ increase in atmospheric temperature enables a switch to basal melt, which then allows a stretch of continuous sliding that peaks just upstream of the grounding-line. The steep ice surface at the bedrock ridge also facilitates an internal feedback in response to a change in precipitation. Rather than any discernible affect on melt and sliding at the bed, an increase in precipitation instead significantly amplifies ice deformation in this area of the glacier. Such disparities in the dynamic response upstream of the grounding-line reveals why the ice volume of Skelton Glacier adjusts at different magnitudes for equivalent environmental perturbations, and highlights the important role of basal melt in influencing ice flux.

The extent of basal melt, and therefore potential for enhanced sliding and ice flux, is greater per step change in atmospheric forcing for thicker and higher velocity outlet glaciers, like Beardmore Glacier (Golledge et al., 2014a). Nevertheless, the importance of this basal sliding mechanism for causing significant mass loss is dependent on the stability of the grounding-line, which is primarily influenced by bed topography (Schoof, 2007) and buttressing (Dupont and Alley, 2005). The main flowpath of Skelton Glacier is currently grounded upstream of a large overdeepening and is therefore not considered vulnerable to retreat, however, the combination of basal sliding and floatation-driven grounding-line retreat may lead to mass loss for the northern arm of Skelton Glacier that currently occupies a deep basin (Golledge et al., 2014a). Furthermore, as ice thickness near the grounding-line of Skelton Glacier is heavily influenced by buttressing at the terminus, thinning or retreat of the Ross Ice Shelf could then lead to enhanced ice flux across the grounding-line through climate-driven basal sliding in the lower reaches of the glacier until a new steady-state profile is reached.

\subsubsection{Glacier dynamics under past climates}

Exploring Skelton Glacier under different climatic conditions provides insight into its potential steady-state dynamics and configurations. Modelling of Skelton Glacier predicts minimal differences in its upper reaches but large topographically-controlled variations in the configuration and behaviour downstream for contrasting past climates. This is in agreement with other studies that suggest that the upper reaches of these glaciers remain stable (e.g. Sugden et al., 1993), whereas the lower reaches undergo dynamic variations in ice geometry (e.g. Naish et al., 2009; Golledge and Levy, 2011).

A terrestrial, fully-grounded Skelton Glacier during the Pliocene interglacials would have likely had sufficient basal melt to slide at its bed along most of its length. As with tem- 
perature glaciers today (e.g. Oerlemans et al., 2013), greatest velocities occurred near its terminus where the surface steepens sharply. The surface profile was likely very different during colder and buttressed episodes, when a very low gradient surface existed. However, despite similar simulated glacier geometries during Pliocene glacials and the LGM, slight differences in environmental conditions produced distinguishable basal conditions and dynamics. Skelton Glacier likely behaved much like today during Pliocene glacials, with basal melt occurring in the deeper basins and along-flow acceleration manifested in the confined, lower reaches of the glacier, peaking at the bedrock ridge. As a result of lower atmospheric temperatures during the LGM, basal melt likely only occurred in the lower basin, near Halfway Nunatak, and together with the low surface gradient, minimal ice velocities could be reached (Golledge and Levy, 2011). Only under Pliocene conditions, when a significantly warmer climate existed and ice was sufficiently thicker during glacial episodes, was basal melt possible at the bedrock ridge.

Basal erosion is very much dependent on whether melt occurs at the bed and the magnitude of basal sliding. Therefore, minor erosion is predicted in the upper and lower basins where ice is thick in all climate scenarios, but no erosion is simulated between $\sim 42 \mathrm{~km}$ and $\sim 95 \mathrm{~km}$ during either Pliocene glacials, the LGM or at present. As high basal temperatures and velocities are modelled during Pliocene interglacials, erosion is possible over the bedrock ridge, and peaks where driving stresses and basal shear stresses are high. Nevertheless, the lower surface profile at this time would prevent erosion of the higher elevation nunataks, while marginal areas of the glacier away from the central incised trough would likely experience reduced basal melt and erosion of the bed from thinner ice (Jamieson et al., 2010; Golledge et al., 2013). In mountainous terrain where outlet glaciers are topographically-controlled, the foci of erosion most probably remains fixed over time, however the magnitude of erosion varies depending on the ice flux at those points (Golledge et al., 2014a), and thus the large basins at Skelton Glacier have likely existed throughout the Late Cenozoic and will probably continue to deepen.

\subsection{Conclusion}

As yet, few studies have investigated the sensitivity of Transantarctic Mountain outlet glaciers to environmental forcings (e.g. Johnson and Staiger, 2007; Riger-Kusk, 2011; Golledge et al., 2014a), and the necessity of such studies is made more apparent considering the varied dynamic responses anticipated between glacial systems (e.g. Oerlemans et al., 1998; Hulbe et al., 2008). Here, aerial and satellite derived data were used to provide preliminary interpretations of the present-day flow regime of Skelton Glacier, and 1 -d flowline modelling then allowed a quantitative prediction of along-flow dynamics un- 
der modern and hypothetical conditions. The modern flow of Skelton Glacier is governed primarily by surface slope, which responds to reduced valley width and large bed undulations, much like other Transantarctic Mountain glaciers (Kavanaugh and Cuffey, 2009; Golledge and Levy, 2011). However, sensitivity experiments carried out this study reveal the importance of basal sliding for maintaining ice flux. Specifically, Skelton Glacier is shown to be most susceptible to perturbations in atmospheric temperature, outside potential buttressing effects.

Under past climates, high magnitude dynamic change occurred in the lower reaches of the glacier. This was primarily the result of the presence or absence of buttressing at the terminus as well as contrasting atmospheric temperatures, with both effects influenced heavily by the topography. It is likely that basal sliding and at least minor erosion occurred in the two overdeepened basins in the lower reaches of the glacier, either side of the bedrock ridge, under all glacial and interglacial climates during the Pliocene and Quaternary. However, near-continuous warm-based, sliding was only possible during peak Pliocene warming.

These findings have implications for the application of surface-exposure dating. Importantly, the central line of nunataks adjacent to the main flow path of Skelton Glacier (Névé Nunatak, Halfway Nunatak, Clinker Bluff) were likely only covered by ice during glacial episodes, primarily as a result of buttressing by grounded ice in the Ross Sea. Under these climatic conditions, bedrock erosion of the nunataks was unlikely. Conversely, at times of high bedrock erosion, for example during Pliocene interglacials, a much thinner Skelton Glacier likely existed that left the nunataks exposed. It can therefore be inferred that negligible bedrock erosion of the nunataks occurred during the Pliocene or Quaternary. Modelling also revealed that only subtle surface elevation changes occurred in the upper reaches of the glacier, which suggests potential difficulty in distinguishing between past ice surface fluctuations (e.g. Staiger et al., 2006; Lilly et al., 2010; Joy et al., 2014). Despite these implied limitations, large surface elevation changes could be captured by surface-exposure dating in the dynamic lower reaches of Skelton Glacier. Fresh erratics recording the LGM and subsequent deglaciation could potentially be found here, having been eroded from the upper basin $(\sim 20-40 \mathrm{~km})$ or lower basin $(\sim 95-115 \mathrm{~km})$ under thicker, more erosive ice. 


\section{Chapter 5}

\section{Complex exposure history of nunataks at Skelton Glacier since the Miocene revealed from multiple-isotope cosmogenic nuclide dating}

\subsection{Introduction}

The behaviour of Antarctic ice sheets is likely linked to orbitally-driven changes in Earth's climate (e.g. Raymo et al., 2006; Huybers, 2009). In the western Ross Sea, sediment cores from the continental-shelf record such ice sheet behaviour during the Miocene, Pliocene and Pleistocene (Naish et al., 2001, 2009; McKay et al., 2012). Numerical ice sheet modelling indicates that these fluctuations in grounded ice extent primarily reflect changes in the configuration of the West Antarctic Ice Sheet (WAIS) since at least the Pliocene (Pollard and DeConto, 2009). Meanwhile, the East Antarctic Ice Sheet (EAIS) may have been a stable, cold-based feature (e.g. Sugden et al., 1993; Siegert et al., 2008), or may have undergone long-term fluctuations in its geometry (e.g. Barrett et al., 1992; Lilly et al., 2010). In the Transantarctic Mountains, East Antarctic outlet glaciers that feed into the Ross Embayment record both prolonged ice surface stability (e.g. Staiger et al., 2006; Di Nicola et al., 2012; Balco et al., 2014) and repeated changes in ice surface elevation (e.g. Di Nicola et al., 2009; Joy et al., 2014). The extent to which these outlet glaciers fluctuated with grounded ice in the Ross Embayment or maintained stable ice configurations, therefore, remains uncertain.

Past ice sheet thickening in the western Ross Embayment is best recorded during the Last Glacial Maximum (LGM) (Anderson et al., 2014). Along the front of the Transantarctic Mountains, the ice sheet surface sloped from $\sim 1400 \mathrm{~m}$ asl near the interior (Todd et al., 2010) to $\sim 400 \mathrm{~m}$ asl in Northern Victoria Land (Orombelli et al., 1990), towards the LGM grounding-line. During this time, outlet glaciers likely thickened in their lower reaches, 'buttressed' by grounded ice in the Ross Sea (e.g. Bockheim et al., 1989; Denton and Hughes, 2000). However, more evidence is required to determined how much thickening occurred and how far this was propagated upstream.

In this chapter, I investigate the long-term history of Skelton Glacier in the Transantarctic Mountains that feeds into the Ross Embayment (Figure 5.1). An assessment of the present-day dynamics of this outlet glacier, along with glacier flowline modelling, suggests that nunataks at least in the lower reaches of Skelton Glacier were overridden by ice 
at the LGM (Chapter 4), however chronological evidence is required to test this hypothesis. Skelton Glacier is fed by ice from the East Antarctic plateau, and therefore changes in the configuration of the EAIS may be reflected in ice surface elevation changes at upstream nunataks. Alternatively, changes in the ice surface at these nunataks may represent high-magnitude, orbitally-paced fluctuations in the extent of grounded ice in the Ross Embayment. Skelton Glacier is well placed to test this idea, as it was a primary source of ice that was delivered to the ANDRILL (AND-1B) core site recording these ice sheet oscillations, since the Early Pliocene (Talarico and Sandroni, 2009; Talarico et al., 2012).

This study seeks answers to the following questions:

1. Did the lower reaches of Skelton Glacier thicken at the LGM?

2. Has Skelton Glacier experienced long-term stability or did its nunataks undergo repeated periods of burial and exposure during the Late Cenozoic?

3. Can past changes in the geometry of Skelton Glacier be attributed to orbitally-paced ice sheet cycles?

I attempt to answer these questions using surface-exposure dating, which is well-suited to investigating former ice surface elevation changes (e.g. Stone et al., 2003; Balco, 2011). This technique also has the potential to record long-term exposure-burial histories using multiple isotopes (e.g. Lilly et al., 2010; Balco et al., 2014), particularly because bedrock samples often exhibit complex exposure histories in Antarctica, as a result of insufficient erosion during periods of ice cover (e.g. Hein et al., 2014).

\subsubsection{Study area}

Skelton Glacier flows from the East Antarctic plateau through the Transantarctic Mountains to the Ross Embayment, with its dynamics influenced by valley width and bed undulations (Chapter 4). A central line of nunataks separates the two main flow paths (Clinker Bluff, Halfway Nunatak, Névé Nunatak) (Figure 5.1). The modern grounding-line occurs at the head of an overdeepened inlet, $\sim 350$ vertical metres below the top of Clinker Bluff. Modelling indicated that these nunataks were likely covered by ice during glacial periods and exposed during interglacial periods, however, with only minor bedrock erosion predicted (Chapter 4). We would therefore expect rock surfaces to preserve a long-term signal of burial and exposure

The bedrock underlying the Skelton Glacier catchment is comprised of a metasedimentary basement (Skelton Group), overlain by sandstone sedimentary sequences (Beacon 


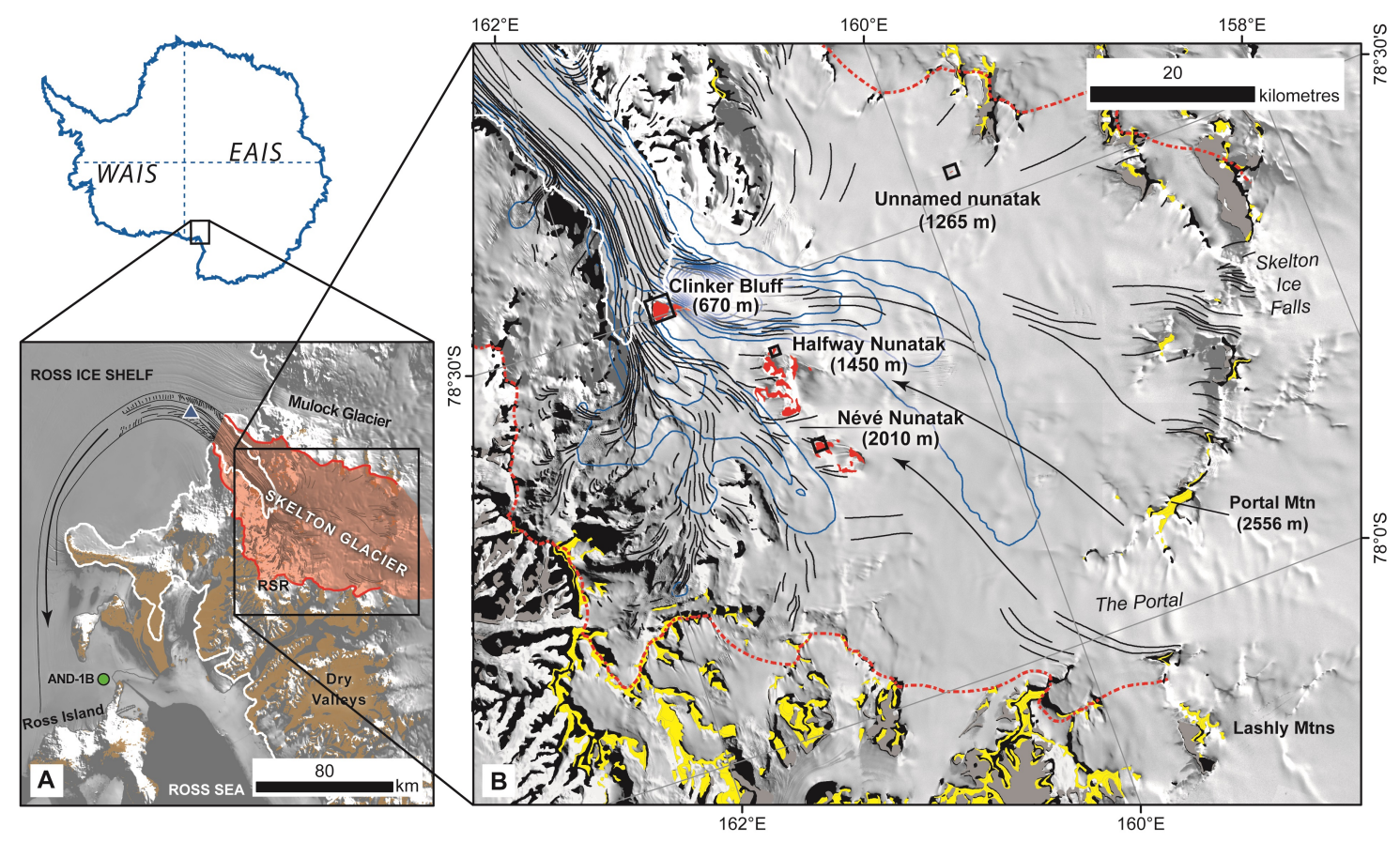

Figure 5.1 Overview of Skelton Glacier and nunataks targeted for surface-exposure dating. A) The catchment of Skelton Glacier is highlighted with respect to the modern Ross Ice Shelf, Royal Society Range (RSR), ice-free areas (brown), and the ANDRILL core site (AND-1B; green circle). Skelton Glacier delivers ice to the core site today, indicated by an arrow, and likely has done since at least the Pliocene (Talarico et al., 2012). Long-term variations in the presence of grounded ice at the mouth of Skelton Glacier are obtained from an ice sheet model (blue triangle; Pollard and DeConto, 2009). The present-day grounding-line is denoted by a white line. B) Ice flows from the East Antarctic plateau, through Skelton Névé to the grounding-line, highlighted with mapped flow-stripes (black lines) and ice velocity contours (at $25 \mathrm{~m} \mathrm{a}^{-1}$ intervals; blue lines) (Rignot et al., 2011). Nunataks targeted for surface-exposure dating are shown in red and specific sample sites are identified with boxes (displayed in Figure 5.2). Sandstone erratics are found on Halfway and Névé Nunataks derived from upstream; Beacon Supergroup sandstone outcrops are shown in yellow (all other exposed bedrock and drift is shown in black and grey respectively) and possible travel paths of erratics are illustrated with arrows.

Supergroup), which are intruded by dolerite dykes and sills (Ferrar Supergroup) (Gunn and Warren, 1962). Névé Nunatak is made up of dolerite, Clinker Bluff is granite and Halfway Nunatak is comprised of both dolerite and granite. Sandstone is exposed in ice-free areas surrounding the névé, including at upstream Portal Mountain and Lashly Mountains, as well as an unnamed nunatak to the south (Figure 5.1).

Evidence of subaerial weathering and past overriding of ice is evident at each of these nunataks. Extensive, long-term surface weathering is suggested by granite surfaces that are heavily spalled, and large areas of exposed dolerite that are heavily fractured. However, past glacial activity is also apparent, as glacially-polished granite surfaces at Clinker Bluff and striated sandstone surfaces at the unnamed nunatak, as well as perched boul- 
ders, erratic cobbles and glacial till at Halfway and Névé Nunataks. Unlike the margins of other Transantarctic Mountain glaciers (e.g. Bockheim et al., 1989; Denton and Marchant, 2000; Di Nicola et al., 2009; Joy et al., 2014), no clear drift limits, moraines or erosional/weathering trimlines exist. Till is largely thin and compacted, however, patterned ground also occurs on the low gradient nunatak slopes. Sub-angular clasts (including boulders $>1 \mathrm{~m}$ in diameter) can be found strewn over the surface, but most of these are locally-derived. Erratic sandstone clasts, sourced from upstream (Figure 5.1), are less common.

The geomorphology observed at the central nunataks of Skelton Glacier suggests both the preservation of weathered relict surfaces and the relatively recent deposition of clasts during a phase of largely non-erosive ice cover. This evidence is typical of cold-based ice that has limited erosive potential, and has been identified elsewhere in the Transantarctic Mountains (Atkins et al., 2002; Lloyd-Davies et al., 2009; Atkins, 2013). Minor erosion is similarly predicted alongside these nunatak sites over the Pliocene and Quaternary from glacier modelling (Chapter 4). This suggests that the bedrock surfaces on these nunataks may have been preserved since the Miocene. Erratic clasts observed at these nunataks were likely sourced from near to, and upstream of, The Portal (Figure 5.1), where thicker ice in an upstream basin facilitated basal erosion (Chapter 4). Glacially-deposited erratics, therefore, have the potential to record a thicker Skelton Glacier in the relatively recent geological past, while bedrock surfaces may record a complex glacial history, representing cold-based glacier activity over many past glaciations during the Late Cenozoic.

\subsection{Surface-exposure dating}

\subsubsection{Method}

Surface-exposure dating is used to reveal the glacial history of the catchment. In particular, this approach seeks to constrain the ice surface elevation during the last glacial cycle and previous glacial-interglacial cycles, as suggested by the geomorphology and offshore sediment cores downstream of Skelton Glacier. The central line of nunataks were targeted to provide an upstream transect along-side the main flow path of the glacier, thereby most likely to represent dynamic ice surface elevations from the modern grounding-line to the upper névé. In order to provide additional spatial coverage of past ice thickness changes within the catchment, the unnamed nunatak was also targeted (Figure 5.1). At each site, samples were collected near to ( \pm 20 vertical $\mathrm{m}$ ) the present-day upstream ice surface so that changes in surface elevation were comparable between nunataks. Modelling suggested that the ice surface likely existed at or below this modern elevation during inter- 
glacials and above this elevation during glacial periods, since at least the Pliocene (Chapter 4), and therefore samples collected here should reflect these glacier fluctuations.

In total, ten samples were collected for surface-exposure dating, from nunatak areas where there was evidence of glacial erosion and deposition. This includes 7 erratic sandstone cobbles on thin, compacted till that was draped over bedrock at Névé Nunatak, an isolated unweathered sandstone erratic boulder on bedrock at Halfway Nunatak, a striated sandstone bedrock surface at the unnamed nunatak, and a glacially-polished granite bedrock surface at Clinker Bluff (Figure 5.2, Table 5.1). As these samples are sandstone and granite, the quartz content is relatively high. Several nuclides can accumulate in quartz when exposed to cosmic rays (Chapter 3$)$, two of which $\left({ }^{26} \mathrm{Al}\right.$ and $\left.{ }^{10} \mathrm{Be}\right)$ are used here to investigate the exposure history of the samples.

Typically, ${ }^{10} \mathrm{Be}$ is used in Antarctica to estimate the age of surface exposure as a result of ice sheet thinning during the last deglaciation (e.g. Stone et al., 2003; Bentley et al., 2010; Balco and Schaefer, 2013; Johnson et al., 2014). However, this single isotope approach relies on the exposed surface having been sufficiently eroded during glacial cover, with no (measureable) inherited nuclide accumulation from a previous episode of exposure. In Antarctica, cold-based, non-erosive ice often fails to sufficiently erode rock surfaces, resulting in exposure chronologies that are internally inconsistent (Hein et al., 2011). To overcome this issue, exposure ages are commonly derived from both ${ }^{10} \mathrm{Be}$ and ${ }^{26} \mathrm{Al}$ (e.g. Mackintosh et al., 2007; Lilly et al., 2010; Storey et al., 2010; White et al., 2011b; Hein et al., 2014), allowing for the investigation of possible complex exposure. Erratic clasts are often sourced from subglacial locations without prior exposure, but in some situations may have been previously exposed, and therefore may or may not show complex exposure. As bedrock surfaces are exposed and buried in situ, they often exhibit complex exposure wherever cold-based ice occurs. As cold-based activity is interpreted the Skelton Glacier nunataks, ${ }^{26} \mathrm{Al}$ is additionally measured here.

Samples were prepared and beryllium and aluminium were extracted according to the methods described in Chapter 3. For beryllium $\left({ }^{9} \mathrm{Be}\right.$ and $\left.{ }^{10} \mathrm{Be}\right)$, all samples were measured relative to the ETH Zurich in-house standard S2007N, and then to the ICN 01-5-1 standard (Nishiizumi et al., 2007), and were then corrected with procedural blanks (mean ${ }^{10} \mathrm{Be} /{ }^{9} \mathrm{Be}$ ratio of $\left.6.5 \times 10^{-15}\right)$. For aluminium $\left({ }^{26} \mathrm{Al}\right)$, samples were measured relative to the ETH Zurich in-house standard ZAL94N and subsequently normalised to the KN-014-1 standard (Nishiizumi, 2004). Meanwhile, stable aluminium $\left({ }^{27} \mathrm{Al}\right)$ required independent measurement, which was carried out at VUW and GeoForschungsZentrum (GFZ), corrected with a caesium spike and checked with standard addition. The final ${ }^{26} \mathrm{Al}$ concentrations, corrected with VUW and GFZ aluminium measurements, were found to be 
consistent between laboratories (Figure 5.3, Table 5.2), overlapping at 1 sigma despite GFZ-derived mean concentrations $4 \%$ higher on average. This inter-laboratory analysis provides confidence that these concentrations have been accurately estimated.
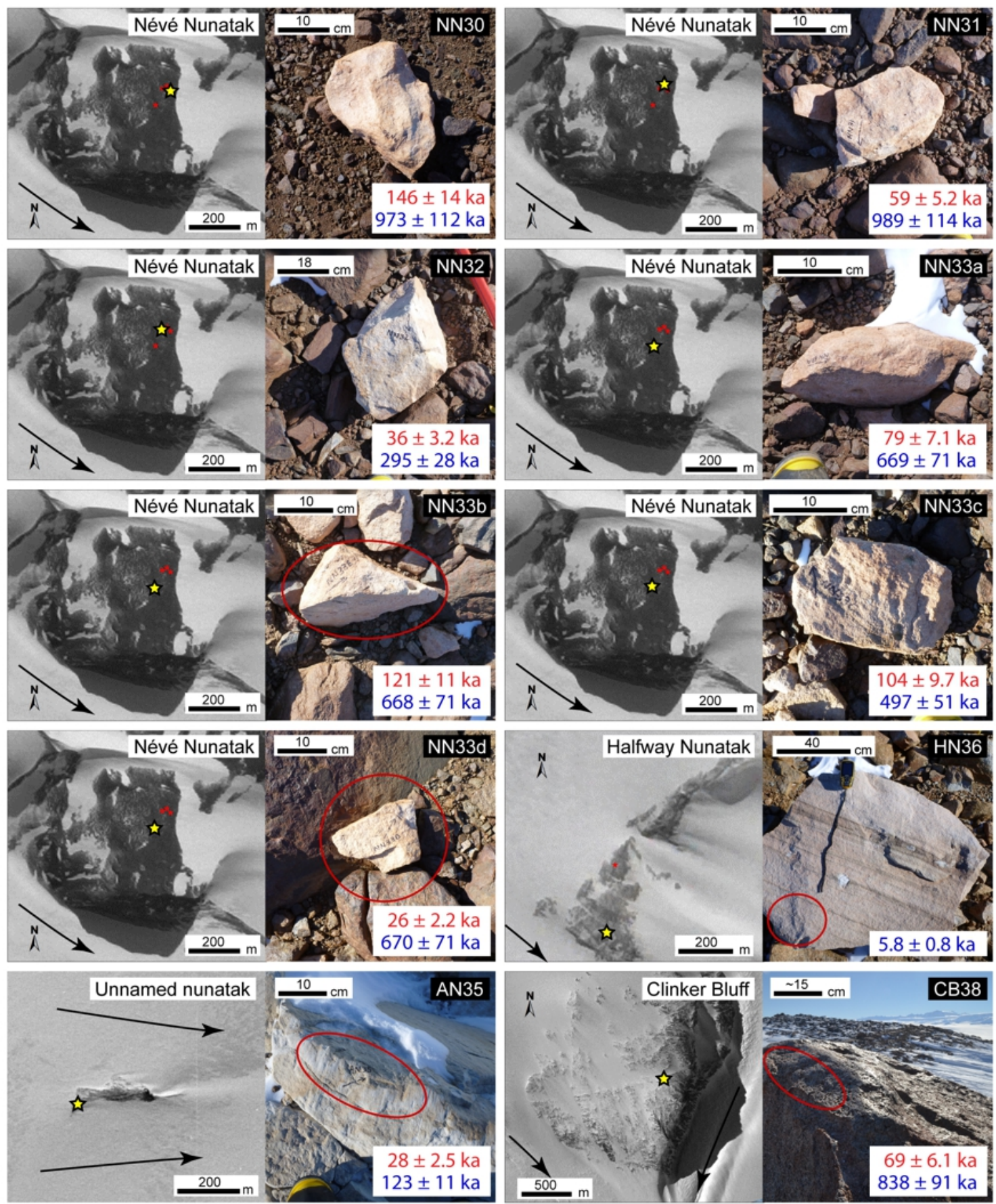

Figure 5.2 Samples collected at Skelton Glacier nunataks. The location of each sample is shown by a yellow star on an aerial photograph. The flow path of Skelton Glacier is highlighted with an arrow. Samples are all sandstone erratics, with AN35 and CB38 sandstone and granite bedrock respectively. Apparent surface-exposure ages are shown for ${ }^{26} \mathrm{Al}$ (red) and ${ }^{10} \mathrm{Be}$ (blue), calculated here using the Lal/Stone (time-dependent) production rate scaling scheme. 
Table 5.1 Details of samples from Skelton Glacier nunataks.

\begin{tabular}{lcccccccc}
\hline Sample ID & Latitude & Longitude & $\begin{array}{c}\text { Altitude } \\
(\mathbf{m} \text { asl) }\end{array}$ & $\begin{array}{c}\text { Sample } \\
\text { lithology }\end{array}$ & Sample position & $\begin{array}{c}\text { Thickness } \\
(\mathbf{c m})\end{array}$ & $\begin{array}{c}\text { Density } \\
\left(\mathbf{g ~ c m}^{2}\right)\end{array}$ & $\begin{array}{c}\text { Shielding } \\
\mathbf{c o r r e c t i o n}^{-}\end{array}$ \\
\hline NN30 & -78.3051 & 160.9898 & 1355 & Sandstone & Cobble on till veneer & 6.0 & 2.2 & 0.999183 \\
NN31 & -78.3053 & 160.9917 & 1332 & Sandstone & Cobble on compacted till & 6.0 & 2.2 & 0.999183 \\
NN32 & -78.3054 & 160.9909 & 1331 & Sandstone & Cobble on till & 2.5 & 2.2 & 0.998310 \\
NN33a & -78.3059 & 160.9895 & 1335 & Sandstone & Cobble on compacted till & 6.0 & 2.2 & 0.991466 \\
NN33b & -78.3059 & 160.9895 & 1335 & Sandstone & Cobble on till and bedrock & 6.5 & 2.2 & 0.999184 \\
NN33c & -78.3059 & 160.9895 & 1335 & Sandstone & Cobble on till & 5.0 & 2.2 & 0.995398 \\
NN33d & -78.3059 & 160.9895 & 1335 & Sandstone & Cobble on till and bedrock & 7.0 & 2.2 & 0.999056 \\
\hline AN35 & -78.5474 & 159.7335 & 1262 & Sandstone & Striated bedrock & 3.5 & 2.2 & 0.999885 \\
\hline HN36 & -78.4243 & 161.0501 & 886 & Sandstone & Boulder perched on bedrock & 5.0 & 2.2 & 0.999984 \\
\hline CB38 & -78.5063 & 161.5931 & 665 & Granite & Edge of polished bedrock & 4.0 & 2.7 & 0.933929 \\
\hline
\end{tabular}

Samples in bold are from bedrock.

Table 5.2 Skelton Glacier sample concentrations.

\begin{tabular}{|c|c|c|c|c|c|c|c|c|c|c|c|c|c|}
\hline $\begin{array}{l}\text { Sample } \\
\text { ID }\end{array}$ & Lab ID & $\begin{array}{c}\text { Final } \\
\text { quartz } \\
\text { weight }(\mathrm{g})\end{array}$ & $\begin{array}{l}{ }^{9} \mathbf{B e} \\
\text { carrier } \\
(\mathbf{m g})\end{array}$ & ${ }^{10} \mathbf{B e} /{ }^{9} \mathbf{B e}$ & $\begin{array}{l}1 \sigma \\
(\%)\end{array}$ & $\begin{array}{c}{ }^{10} \text { Be conc. } \\
\left(\text { atoms } \mathbf{~ g ~ a ~}^{-1}\right)\end{array}$ & $\begin{array}{c}\mathbf{1} \sigma \\
\left(\text { atoms }_{\mathbf{g} ~ \mathbf{a}^{-1}}\right)\end{array}$ & ${ }^{26} \mathbf{A} \mathbf{l} /{ }^{27} \mathbf{A l}$ & $\begin{array}{l}1 \sigma \\
(\%)\end{array}$ & $\begin{array}{l}{ }^{26} \mathbf{A l} \text { conc. } \\
\left(\text { atoms } \mathbf{~ g ~ a ~}{ }^{-1} \text { ) }\right. \\
(\text { GFZ) }\end{array}$ & $\begin{array}{c}1 \sigma \\
\left(\text { atoms g a }^{-1}\right) \\
(\text { GFZ) }\end{array}$ & $\begin{array}{c}{ }^{26} \mathbf{A l} \text { conc. } \\
\left(\text { atoms g a }{ }^{-1}\right) \\
(\mathbf{V U W})\end{array}$ & $\begin{array}{c}\mathbf{1} \sigma \\
\left(\text { atoms g a }^{-1}\right) \\
(\mathbf{V U W})\end{array}$ \\
\hline NN30 & RV01, RV42 & 56.24 & 0.1861 & $6.724 \mathrm{E}-11$ & 3.0 & $1.4548 \mathrm{E}+07$ & $4.36 \mathrm{E}+05$ & $1.59 \mathrm{E}-11$ & 2.8 & $1.7325 \mathrm{E}+07$ & $4.90 \mathrm{E}+05$ & $1.6316 \mathrm{E}+07$ & $5.34 \mathrm{E}+05$ \\
\hline NN31 & RV06, RV45 & 26.40 & .1680 & 7.394E-11 & 3.0 & $1.4418 \mathrm{E}+07$ & $33 \mathrm{E}+$ & $1.51 \mathrm{E}-11$ & & 7.1239E+06 & $1.57 \mathrm{E}+05$ & $6.7606 \mathrm{E}+06$ & $1.49 \mathrm{E}+05$ \\
\hline NN32 & RV07, RV46 & 34.02 & 1672 & $2.734 \mathrm{E}-11$ & 3.0 & & & & & 4.4864E+06 & $1.22 \mathrm{E}+05$ & $4.2895 \mathrm{E}$ & $1.74 \mathrm{E}+05$ \\
\hline NN33a & RV05, R & & 1681 & $5.160 \mathrm{~B}$ & & & & 1.76 & & 9.4607 & $1.94 \mathrm{E}$ & 9.06 & $1.86 \mathrm{E}+$ \\
\hline NN33b & & & 1677 & 5.75 & 3. & & .14 & $1.50 \mathrm{E}$ & & 1.42741 & $2.95 \mathrm{E}+05$ & 1.39 & $5.09 \mathrm{E}+05$ \\
\hline NN33c & RV08, RV49 & 57.40 & 0.1677 & 4.163E-11 & 3.2 & $8.1786 \mathrm{E}+06$ & $2.62 \mathrm{E}+05$ & $1.29 \mathrm{E}-11$ & 2.7 & $1.2411 \mathrm{E}+07$ & $3.35 \mathrm{E}+05$ & 1.1991 & $3.24 \mathrm{E}+05$ \\
\hline NN33d & RV09, RV50 & 12.30 & 0.1677 & $5.440 \mathrm{E}-11$ & 3.0 & $1.0483 \mathrm{E}+07$ & $3.14 \mathrm{E}+05$ & $1.62 \mathrm{E}-11$ & 1.9 & $3.1582 \mathrm{E}+06$ & $6.15 \mathrm{E}+04$ & $3.1979 \mathrm{E}+06$ & $8.80 \mathrm{E}+04$ \\
\hline AN35 & RV02, RV51 & 61.03 & 0.1784 & $1.033 \mathrm{E}-11$ & 3.0 & $2.1274 \mathrm{E}+06$ & $6.38 \mathrm{E}+04$ & $5.36 \mathrm{E}-12$ & 2.9 & $3.3351 \mathrm{E}+06$ & $9.55 \mathrm{E}+04$ & $3.1374 \mathrm{E}+06$ & $1.85 \mathrm{E}+05$ \\
\hline HN36 & RV04 & 21.54 & 0.1650 & $1.488 \mathrm{E}-13$ & 9.7 & 7.4664E+04 & $7.60 \mathrm{E}+03$ & - & - & - & - & - & - \\
\hline CB38 & RV24 & 33.80 & 0.2055 & $1.657 \mathrm{E}-11$ & 2.4 & $6.7324 \mathrm{E}+06$ & $1.61 \mathrm{E}+05$ & $2.01 \mathrm{E}-11$ & 2.1 & $4.4393 \mathrm{E}+06$ & $9.10 \mathrm{E}+04$ & $4.1439 \mathrm{E}+06$ & $1.84 \mathrm{E}+05$ \\
\hline
\end{tabular}

Note: ${ }^{26} \mathrm{Al}$ concentrations derived from GFZ and VUW aluminium measurements are indistinguishable (Figure 5.3). Those of GFZ are used hereafter. 


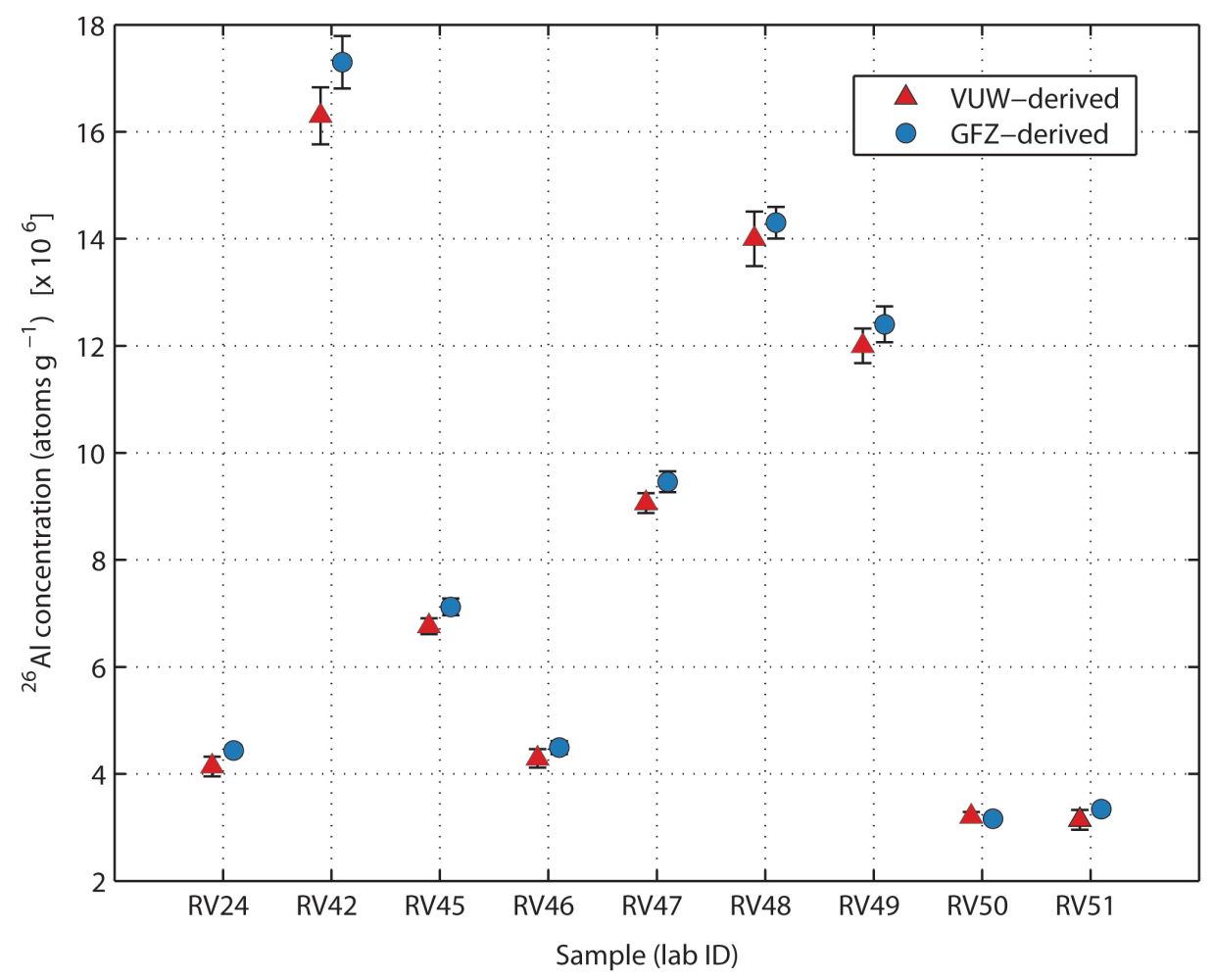

Figure 5.3 Consistent ${ }^{26} \mathrm{Al}$ concentrations derived from VUW and GFZ measurements. Corrected with ${ }^{27} \mathrm{Al}$ measurements, ${ }^{26} \mathrm{Al}$ concentrations overlap between laboratories at $1 \sigma$. On average, GFZ-derived mean concentrations were $4 \%$ higher those from VUW measurements.

\subsubsection{Results}

The concentrations of ${ }^{10} \mathrm{Be}$ and ${ }^{26} \mathrm{Al}$ (Table 5.2), accumulated as a result of cosmic irradiation, represent the exposure time of the rock surface since deglaciation. Surface exposure ages can therefore be estimated given rates of radionuclide production and decay (Chapter 3). Here, exposure ages were calculated for both ${ }^{10} \mathrm{Be}$ and ${ }^{26} \mathrm{Al}$ using the SLHL ${ }^{10} \mathrm{Be}$ production rate $\left(4.49 \pm 0.39\right.$ atoms $\mathrm{g} \mathrm{a}^{-1}$ due to spallation; Stone, 2000; Balco et al., 2008) and constant ${ }^{26} \mathrm{Al} /{ }^{10} \mathrm{Be}$ production rate relationship of 6.75 (Balco et al., 2008), following corrections for topographic shielding and sample thickness (Chapter 3, Table 5.1). Nuclide production varies with time and space, and therefore a range of scaling schemes with different temporal dependence are used; the Lal/Stone time-dependent scheme (Stone, 2000; Nishiizumi et al., 1989) is considered most appropriate for these samples as it accounts for Antarctic-specific air pressure (Chapter 3). The resulting ages are termed 'apparent exposure ages' as they assume continuous exposure since initially exposed and zero surface erosion over that time (Tables 5.3 and 5.4). 
Table 5.3 ${ }^{10} \mathrm{Be}$ apparent surface-exposure ages at Skelton Glacier.

\begin{tabular}{|c|c|c|c|c|c|c|c|c|c|c|c|c|c|}
\hline \multirow{2}{*}{$\begin{array}{l}\text { Sample } \\
\text { ID }\end{array}$} & \multirow{2}{*}{$\begin{array}{c}\mathbf{P}_{S}(\text { muons }) \\
\left(\text { atoms g a }^{-1}\right)\end{array}$} & \multirow{2}{*}{$\begin{array}{c}\mathbf{P}_{S} \text { (spallation) } \\
\left(\text { atoms g a }^{-1}\right)\end{array}$} & \multirow{2}{*}{$\begin{array}{c}\text { Internal uncertainty } \\
(1 \sigma)(\mathbf{y r s})\end{array}$} & \multicolumn{2}{|c|}{ Lal/Stone (non t-d) *十 } & \multicolumn{2}{|c|}{ Desilets \& Zreda \# } & \multicolumn{2}{|c|}{ Dunai $\S$} & \multicolumn{2}{|c|}{ Lifton et al. $\star$} & \multicolumn{2}{|c|}{ Lal/Stone $(\mathbf{t}-d) * \dagger \ddagger$} \\
\hline & & & & Age (yrs) & Uncertainty & Age (yrs) & Uncertainty & Age (yrs) & Uncertainty & Age (yrs) & Uncertainty & Age (yrs) & Uncertainty \\
\hline NN30 & 0.313 & 18.93 & 36335 & 945336 & 111071 & 1013657 & 160609 & 986805 & 154565 & 926043 & 121347 & 972592 & 112071 \\
\hline NN31 & 0.31 & 18.58 & 37021 & 961258 & 113400 & 1032154 & 164339 & 1004796 & 158128 & 942916 & 124096 & 989084 & 114451 \\
\hline NN32 & 0.313 & 19 & 9314 & 288500 & 28519 & 306111 & 40245 & 299377 & 39126 & 284029 & 31442 & 295464 & 28485 \\
\hline NN33a & 0.311 & 18.48 & 23100 & 651444 & 70758 & 695294 & 101155 & 678406 & 97842 & 639946 & 77694 & 668762 & 71040 \\
\hline NN33b & 0.31 & 18.56 & 23047 & 650358 & 70618 & 694126 & 100952 & 677270 & 97647 & 638882 & 77541 & 667644 & 70898 \\
\hline NN33c & 0.311 & 18.68 & 17556 & 484881 & 50788 & 516033 & 71924 & 504060 & 69742 & 476734 & 55784 & 497208 & 50884 \\
\hline NN33d & 0.31 & 18.5 & 23173 & 653031 & 70963 & 697010 & 101453 & 680072 & 98128 & 641499 & 77917 & 670400 & 71248 \\
\hline AN35 & 0.305 & 17.86 & 3734 & 120691 & 11433 & 128041 & 16088 & 125382 & 15677 & 119418 & 12678 & 123478 & 11395 \\
\hline HN36 & 0.267 & 12.81 & 583 & 5718 & 766 & 6113 & 954 & 6001 & 934 & 5811 & 825 & 5845 & 774 \\
\hline CB38 & 0.247 & 9.8 & 24053 & 815653 & 90713 & 889561 & 134765 & 869867 & 130519 & 825574 & 103640 & 838140 & 91191 \\
\hline
\end{tabular}

$\mathrm{P}_{S}=$ Sample-specific production rate, calculated with Lal/Stone scaling.

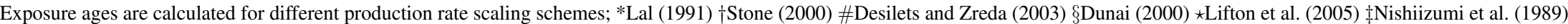

Calculated ages and associated uncertainties should only be considered at 3-4 significant figures.

Table 5.4 ${ }^{26} \mathrm{Al}$ apparent surface-exposure ages at Skelton Glacier.

\begin{tabular}{|c|c|c|c|c|c|c|c|c|c|c|c|c|c|}
\hline \multirow{2}{*}{$\begin{array}{l}\text { Sample } \\
\text { ID }\end{array}$} & \multirow{2}{*}{$\begin{array}{c}\mathbf{P}_{S}(\mathbf{m u o n s}) \\
\left(\text { atoms g a }^{-1}\right)\end{array}$} & \multirow{2}{*}{$\begin{array}{c}\mathbf{P}_{S}(\text { spallation }) \\
\left(\text { atoms g a }^{-1}\right)\end{array}$} & \multirow{2}{*}{$\begin{array}{c}\text { Internal uncertainty } \\
(1 \sigma)(\mathrm{yrs})\end{array}$} & \multicolumn{2}{|c|}{ Lal/Stone (non t-d) $* \dagger$} & \multicolumn{2}{|c|}{ Desilets \& Zreda \# } & \multicolumn{2}{|c|}{ Dunai $\S$} & \multicolumn{2}{|c|}{ Lifton et al. $\star$} & \multicolumn{2}{|c|}{ Lal/Stone $(\mathbf{t}-\mathbf{d}) * \dagger \dagger$} \\
\hline & & & & Age (yrs) & Uncertainty & Age (yrs) & Uncertainty & Age (yrs) & Uncertainty & Age (yrs) & Uncertainty & Age (yrs) & Uncertainty \\
\hline NN30 & 2.617 & 127.73 & 4323 & 142211 & 13944 & 150695 & 19697 & 147387 & 19151 & 139952 & 15386 & 145622 & 13920 \\
\hline NN31 & 2.597 & 125.36 & 1298 & 57223 & 5275 & 60537 & 7482 & 59273 & 7290 & 56532 & 5871 & 58537 & 5249 \\
\hline NN32 & 2.617 & 128.17 & 965 & 34924 & 3234 & 36925 & 4550 & 36163 & 4436 & 34583 & 3597 & 35717 & 3219 \\
\hline NN33a & 2.6 & 124.7 & 1644 & 77168 & 7155 & 81675 & 10178 & 79950 & 9911 & 76148 & 7960 & 78957 & 7122 \\
\hline NN33b & 2.597 & 125.24 & 2593 & 118499 & 11220 & 125574 & 15999 & 122862 & 15564 & 116794 & 12463 & 121306 & 11179 \\
\hline NN33c & 2.605 & 126.05 & 2876 & 101262 & 9685 & 107253 & 13683 & 104958 & 13317 & 99846 & 10722 & 103639 & 9656 \\
\hline NN33d & 2.595 & 124.8 & 495 & 25111 & 2263 & 26539 & 3213 & 25994 & 3133 & 24916 & 2534 & 25679 & 2249 \\
\hline AN35 & 2.55 & 120.46 & 798 & 27520 & 2551 & 29160 & 3589 & 28569 & 3500 & 27385 & 2849 & 28142 & 2540 \\
\hline CB38 & 2.063 & 66.14 & 1426 & 67280 & 6207 & 72273 & 8964 & 70960 & 8757 & 68142 & 7095 & 68817 & 6176 \\
\hline
\end{tabular}

$\mathrm{P}_{S}=$ Sample-specific production rate, calculated with Lal/Stone scaling.

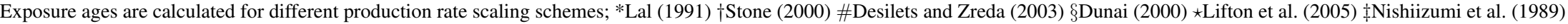

Calculated ages and associated uncertainties should only be considered at 3-4 significant figures. 
The apparent exposure ages at Skelton Glacier are highly variable, with no consistent timing of deglaciation suggested. The youngest ${ }^{10} \mathrm{Be}$ exposure age comes from the relatively unweathered glacial erratic at Halfway Nunatak (HN36), dated to $5.8 \pm 0.8$ ka (Figure 5.2, Table 5.3). This is the only sample that most likely records deglaciation during the last glacial cycle and as it is located close to the present-day glacier margin, it suggests that surface lowering here was near-complete by the mid-Holocene. All other samples provide a ${ }^{10} \mathrm{Be}$ apparent exposure age range from $123 \pm 11 \mathrm{ka}$ to $989 \pm 114 \mathrm{ka}$ (Figure 5.2, Table 5.3), with no strong agreement in ages over that time (Figure 5.4). The age distribution of these samples also fails to reveal any pattern between nunataks or sample type; the ages of erratics at upstream Névé Nunatak range from $\sim 200 \mathrm{ka}$ to $\sim 1.2 \mathrm{Ma}$, while bedrock-derived ages at the unnamed nunatak in the outer névé and at downstream Clinker Bluff are $\sim 120 \mathrm{ka}$ and $\sim 840 \mathrm{ka}$ respectively. The meaning of such a ${ }^{10} \mathrm{Be}$ apparent age distribution, spanning multiple glacial cycles, can be evaluated further with additional ${ }^{26} \mathrm{Al}$ determined exposure ages.

A substantially younger exposure history is suggested by ${ }^{26} \mathrm{Al}$ apparent exposure ages, with all samples dated to $<180 \mathrm{ka}$ (Table 5.4, Figure 5.4). While exposure ages from multiple nunataks coincide with the last glacial period ( $\sim 26-36 \mathrm{ka}, \mathrm{n}=3)$, no samples have matching ${ }^{26} \mathrm{Al}$ and ${ }^{10} \mathrm{Be}$ ages. Again, the apparent age distributions show no pattern between nunataks or between sample types. Together, ${ }^{10} \mathrm{Be}$ and ${ }^{26} \mathrm{Al}$ data indicate that these samples have experienced a complex exposure history with significant cosmogenic inheritance and, therefore, the apparent exposure ages cannot be considered absolute estimates for the timing of deglaciation.

The complex exposure history implied by the apparent exposure ages can be better understood with multiple isotope analysis. Cosmogenic inheritance results from insufficient erosion of a rock surface when overridden or entrained by ice. Nuclide concentrations can undergo radioactive decay during such burial, and the shorter half-life of ${ }^{26} \mathrm{Al}$ (716 ka) relative to ${ }^{10} \mathrm{Be}(1.39 \mathrm{Ma})$ enables faster relative decay and therefore a reduced ${ }^{26} \mathrm{Al} /{ }^{10} \mathrm{Be}$ ratio over this time (Chapter 3 ). The burial and exposure history can, therefore, be evaluated with two-nuclide analysis (Lal, 1991) (Figure 5.5).

A preliminary analysis (Figure 5.5A) reveals that the complex history recorded in these samples represents $>2$ Ma of cumulative burial and $>500 \mathrm{ka}$ of cumulative exposure. The unnamed nunatak (AN35) has experienced the least amount of cumulative exposure, while samples at Clinker Bluff and Névé Nunatak indicate as much as $>10 \mathrm{Ma}$ of exposure. Secondly, several of these samples plot beyond secular equilibrium, in an area termed the "forbidden zone". Two regions exist within this zone; 1) above the steady-state erosion island $\left({ }^{26} \mathrm{Al} /{ }^{10} \mathrm{Be}\right.$ ratios larger than the production ratio), which often indicates measurement error (i.e. matrix interferences in the plasma when measuring ${ }^{27} \mathrm{Al}$ ), and 2 ) below 

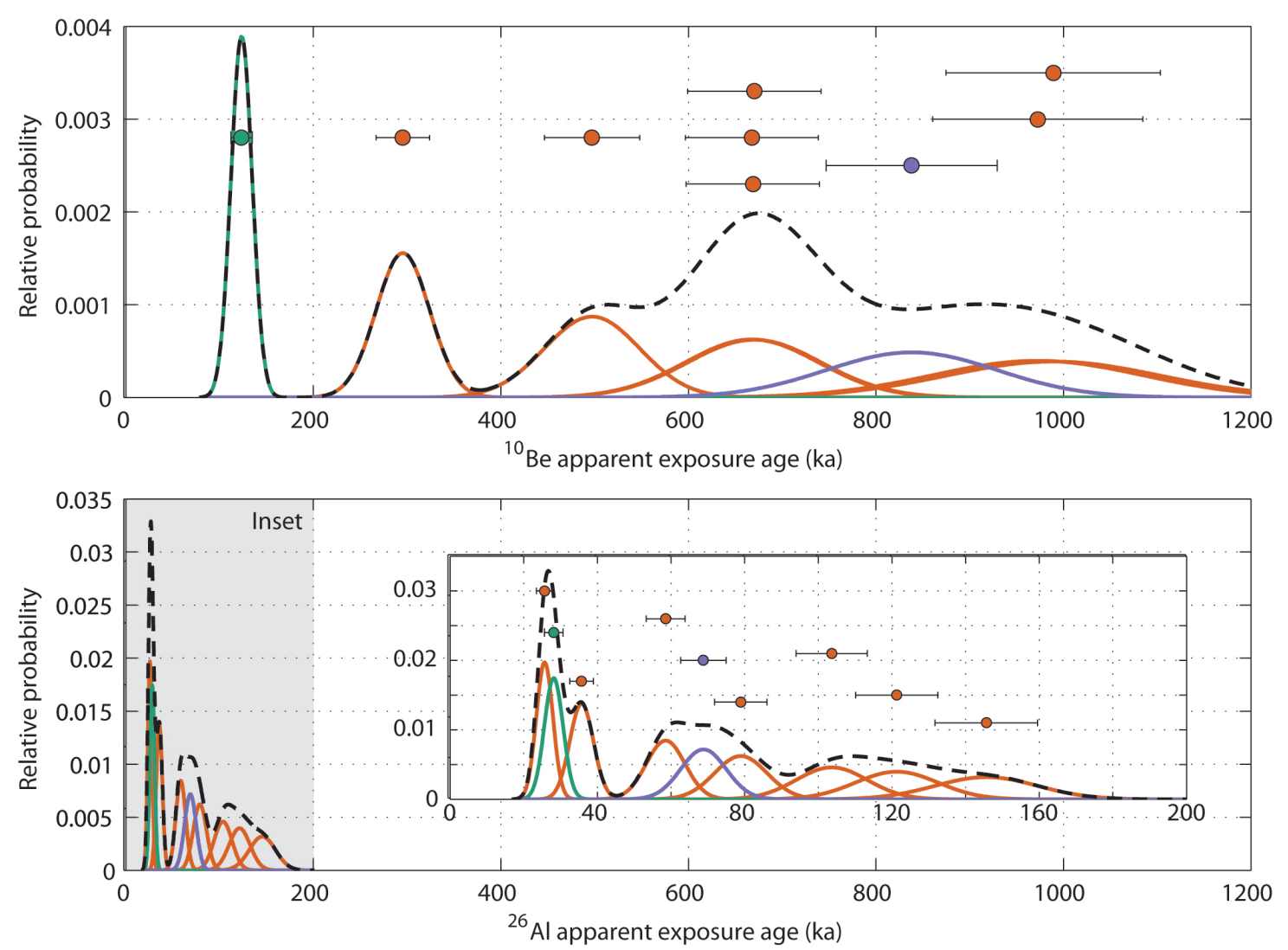

Figure 5.4 Probability plots of apparent ${ }^{10} \mathrm{Be}$ and ${ }^{26} \mathrm{Al}$ exposure ages at Névé Nunatak (orange), the unnamed nunatak (green) and Clinker Bluff (purple). The summed probability is shown with a dashed black line. Ages are also displayed with error bars at $1 \sigma$, plotted without a y-axis. In the upper panel, ${ }^{10} \mathrm{Be}$ apparent exposure ages produce no distinct probability peak that would be suggestive of a common deglaciation date, and instead show a broad range from $\sim 120 \mathrm{ka}$ to $>1 \mathrm{Ma}$. Importantly, ${ }^{26} \mathrm{Al}$ apparent exposure ages (lower panel) are consistently lower, with all ages suggesting deglaciation since $\sim 180 \mathrm{ka}$. Together, these ${ }^{10} \mathrm{Be}$ and ${ }^{26} \mathrm{Al}$ data indicate that each sample has experienced significant cosmogenic inheritance from prior episodes of exposure and, therefore, the apparent exposure ages are not reliable estimates of the last deglaciation.

the island and beyond secular equilibrium (where a surface's nuclide concentration is saturated, decay=production), which is forbidden due to the limits of nuclide production and decay. In theory, such low ${ }^{26} \mathrm{Al} /{ }^{10} \mathrm{Be}$ ratios and high ${ }^{10} \mathrm{Be}$ concentrations in these samples could result from ${ }^{26} \mathrm{Al}$ loss or ${ }^{10} \mathrm{Be}$ gain in the laboratory, however, carrier and standard addition as well as inter-laboratory agreement rule this scenario unlikely.

In applying two-nuclide analysis, the sample production rates over the period of complex exposure are assumed to be those of today (SLHL; Stone, 2000; Balco et al., 2008). While the production ratio between ${ }^{26} \mathrm{Al}$ and ${ }^{10} \mathrm{Be}$ is considered to be constant through time (Balco et al., 2008), the sample production rate of ${ }^{10} \mathrm{Be}$ may have been higher. If so, nuclide concentrations would instead plot prior to secular equilibrium, providing more 


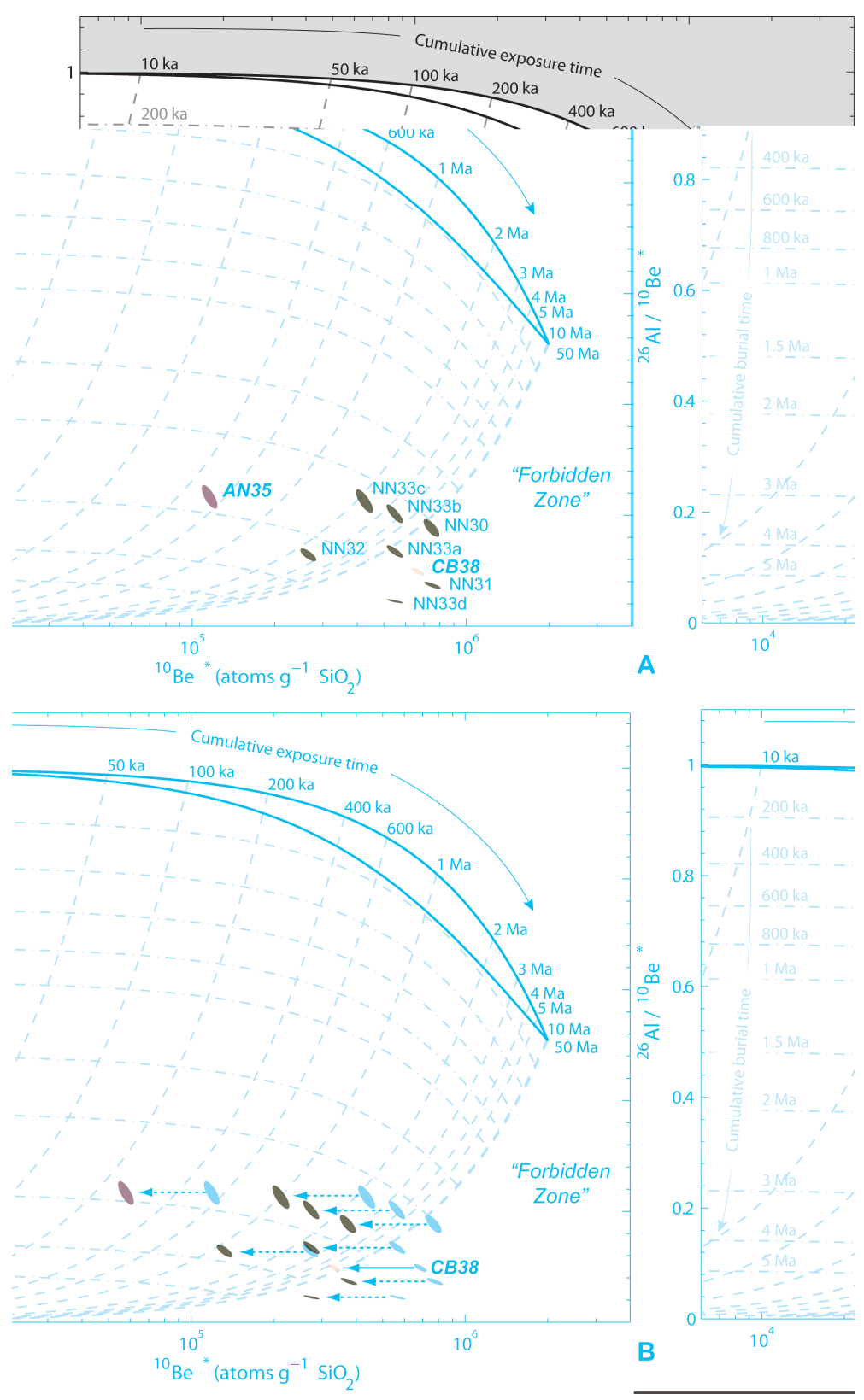

Figure 5.5 Two-nuclide plot showing complex sample exposure history, normalised to SLHL. Cumulative exposure and burial isochrons are shown (dashed and dot-dashed grey lines) together with the steady-state erosion island (solid black lines), within which a simple exposure history is implied. Skelton Glacier sample concentrations $(2 \sigma)$ are plotted and coloured by nunatak. A) Measured ${ }^{10} \mathrm{Be}$ and ${ }^{26} \mathrm{Al}$ concentrations indicate that these samples experienced a long complex history, culminating in $>0.5-10 \mathrm{Ma}$ of minimum cumulative exposure and $>2 \mathrm{Ma}$ of burial. However, most samples plot beyond secular equilibrium (in the "Forbidden zone"), assuming SLHL ${ }^{10}$ Be production rate of 5.1 atoms $\mathrm{g} \mathrm{a}^{-1}$ (Balco et al., 2008), including the bedrock sample of CB38 (purple). B) Using a mean ${ }^{10} \mathrm{Be}$ production rate $2 \mathrm{x}$ that of today (SLHL) over this time, CB38 instead plots realistically within secular equilibrium. See text for discussion. Following this correction, CB38 at Clinker Bluff indicates $\sim 10 \mathrm{Ma}$ of cumulative exposure and $>2.5$ Ma of burial, while erratics at Névé Nunatak (orange) show $>1$ Ma of exposure and $>2.5$ Ma of burial, and AN35 at the unnamed nunatak (green) shows $\sim 300 \mathrm{ka}$ of exposure and $\sim 3$ Ma of burial. 
realistic estimates for the complex histories of these samples (Figure 5.5B); $10 \mathrm{Ma}$ of cumulative exposure and >2.5 Ma of burial is suggested at Clinker Bluff (CB38), while erratics at Névé Nunatak show $>1$ Ma of exposure and $>2.5 \mathrm{Ma}$ of burial, and the unnamed nunatak (AN35) shows $\sim 300$ ka of exposure and $\sim 3$ Ma of burial.

In summary, a single ${ }^{10} \mathrm{Be}$ age from Halfway Nunatak reveals that the most recent surface lowering of Skelton Glacier reached the near-present ice surface by $\sim 5.8 \mathrm{ka}$. Two-nuclide apparent ages $\left({ }^{26} \mathrm{Al}\right.$ and $\left.{ }^{10} \mathrm{Be}\right)$ suggest that Névé Nunatak, Clinker Bluff and the unnamed nunatak were overridden by ice in the past, but that these nunataks experienced a complex exposure history, indicative of cosmogenic inheritance. Such a history involved exposure and burial over hundreds of thousands to tens of millions of years, with an inferred production rate likely higher than that of today.

\subsection{Discussion}

\subsubsection{Skelton Glacier during the last glacial cycle}

Surface-exposure dating suggests that at least the lower reaches of Skelton Glacier were thicker than present at the LGM. Commonly, the youngest exposure age is used in Antarctica to represent the timing of most recent deglaciation (Chapter 3; Stone et al., 2003; Mackintosh et al., 2007; Bentley et al., 2010). Here, the single-nuclide $\left({ }^{10} \mathrm{Be}\right)$ exposure age of $\sim 5.8 \mathrm{ka}$ at Halfway Nunatak represents deglaciation during the last glacial cycle. It implies that the glacier surface was higher prior to the mid-Holocene, but does not help constrain a maximum surface elevation at the LGM. However, in order for the glacier to thicken sufficiently to bury the sample at Halfway Nunatak, modelling indicates that the LGM surface elevation at the mouth of the Skelton Glacier would have been $\sim 700 \mathrm{~m}$ asl (Chapter 4). Together, this evidence therefore supports the thickening of Transantarctic Mountain outlet glaciers in response to buttressing by grounded ice in the Ross Sea (e.g. Bockheim et al., 1989). It also supports a south-north sloping ice sheet surface profile in this region at the LGM, in line with estimates at Hatherton Glacier ( $~ 800 \mathrm{~m}$ asl; Bockheim et al., 1989; Anderson et al., 2004) and Minna Bluff ( $240 \mathrm{~m}$ asl; Denton and Marchant, 2000). Ideally, a greater population of younger (Holocene) ages would be available to give additional strength to this interpretation.

Glacier modelling additionally predicted the overriding of Clinker Bluff and Névé Nunatak at the LGM (Chapter 4), where samples reveal a complex glacial history indicative of cos- 
mogenic inheritance. In this situation, a nuclide inventory accumulates during a period of exposure (i.e. an interglacial) but fails to be completely removed by overriding ice during a glacial period, resulting in an 'inherited' nuclide inventory (Figure 5.6); during a typical Late Pleistocene glacial-interglacial cycle, of $\sim 15 \mathrm{ka}$ of exposure and $\sim 85 \mathrm{ka}$ of burial (Tzedakis et al., 2012), cosmogenic inheritance would result from glacial erosion of $<\sim 3.5 \mathrm{~cm} \mathrm{ka}^{-1}$. In the western Ross Sea, however, ice may have become grounded between $\sim 27{ }^{14} \mathrm{C}$ ka BP (Denton and Marchant, 2000) and $\sim 7{ }^{14} \mathrm{C}$ ka BP (Hall et al., 2004), indicating that the typical duration of a glacial episode in the region could be $\sim 20 \mathrm{ka}$. Under this scenario, at least $14 \mathrm{~cm} \mathrm{ka}^{-1}$ of erosion would be required over this time to remove any cosmogenic inheritance from the previous $\sim 80 \mathrm{ka}$ of exposure. During the LGM, basal erosion of $\sim 0.0005-0.001 \mathrm{~m} \mathrm{a}^{-1}\left(50-100 \mathrm{~cm} \mathrm{ka}^{-1}\right)$ was simulated at overdeepenings in the central trunk of Skelton Glacier, but $<10 \mathrm{~cm} \mathrm{ka}^{-1}$ of erosion was predicted elsewhere along the flow path (Chapter 4). The cosmogenic inheritance recorded with surface-exposure dating indicates that ice at these nunataks, adjacent to the main flowpath, generated $<14 \mathrm{~cm} \mathrm{ka}^{-1}$, and possibly $<3.5 \mathrm{~cm} \mathrm{ka}^{-1}$, of basal erosion at the LGM.

The onset and style of deglaciation following the LGM cannot be determined, however, the exposure age at Halfway Nunatak indicates that glacier thinning likely continued into the Holocene. More specifically, this date suggests that the modern ice surface in the lower reaches of Skelton Glacier was reached by $\sim 5.8 \mathrm{ka}$. This evidence is in contrast to other East Antarctic outlet glaciers, where surface lowering had largely ceased by the early Holocene (e.g. White et al., 2011b), and instead, is more consistent with the chronologies of West Antarctic outlets, which record surface lowering into the mid/late-Holocene (e.g. Stone et al., 2003; Bentley et al., 2010; Johnson et al., 2014). The timing of glacier thinning is heavily dependent on the style of deglaciation downstream. At Skelton Glacier, ice discharge and surface lowering likely occurred in response to the retreat of grounded ice in the Ross Embayment (e.g. Bockheim et al., 1989; Anderson et al., 2004). Such grounding-line retreat is recorded just north of Ross Island by $\sim 10 \mathrm{ka}$ (calibrated ${ }^{14} \mathrm{C}$; Mckay et al., 2008) and then south of Skelton Glacier, to Hatherton Glacier, at $~ 7.9 \mathrm{ka}$ (6.8 calibrated ${ }^{14} \mathrm{C} \mathrm{ka}$, extrapolated to the glacier mouth with glacier modelling; Bockheim et al., 1989; Anderson et al., 2004), occurring in a 'swinging gate' pattern along the front of the Transantarctic Mountains (Conway et al., 1999). This implies that groundingline recession past the mouth of Skelton Glacier occurred sometime between $\sim 10 \mathrm{ka}$ and $\sim 7.9 \mathrm{ka}$, at least $\sim 1,500$ years prior to the final mid-Holocene surface lowering that is recorded at Halfway Nunatak.

The lag between the recorded timings in deglaciation may represent uncertainties in the dating techniques and/or the delayed upstream response of Skelton Glacier. A single surface-exposure age is a relatively poor constraint on the timing of deglaciation, 


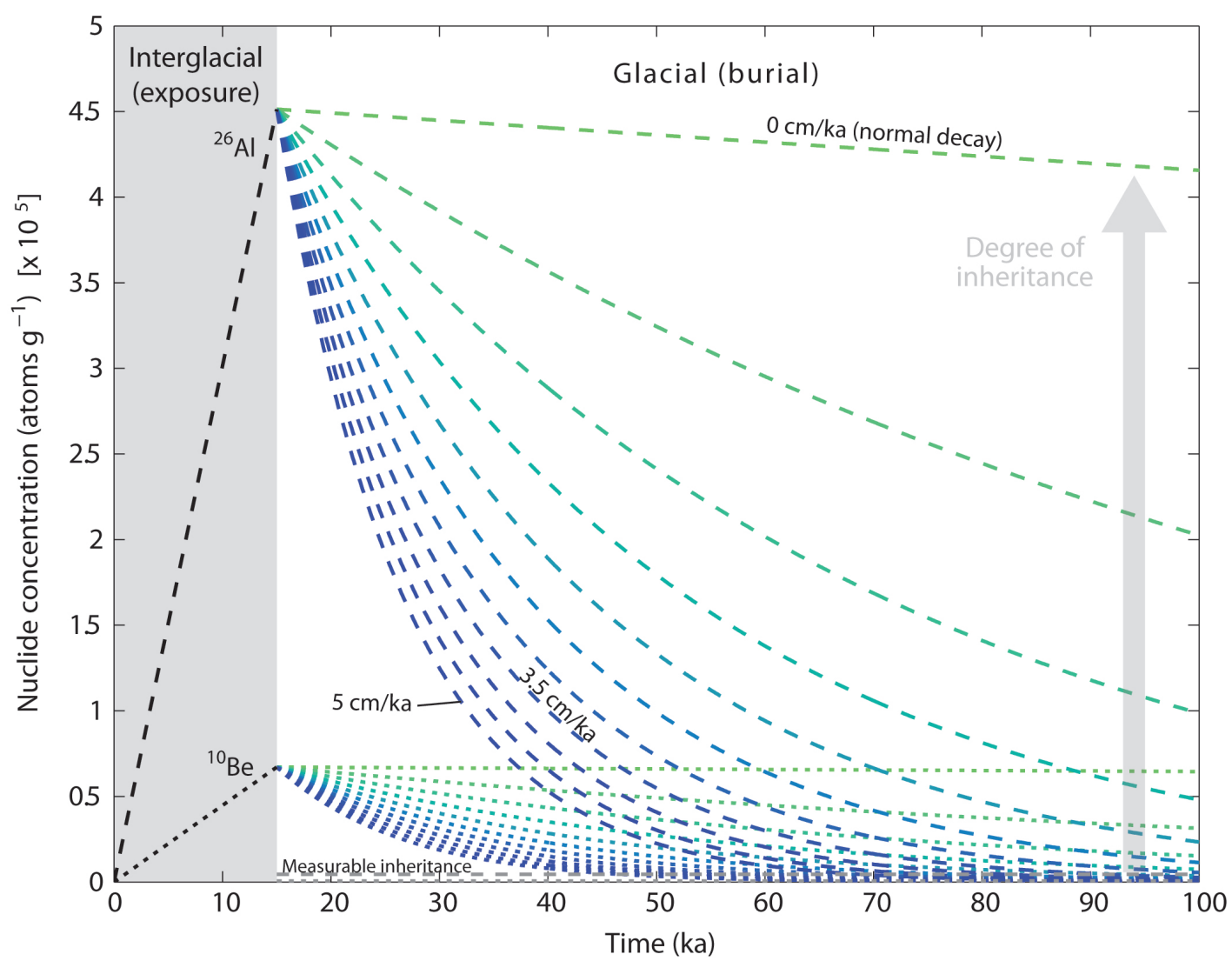

Figure 5.6 Evolution of nuclide concentrations during a typical Late Pleistocene glacial-interglacial cycle. Concentrations are modelled (at SLHL) for ${ }^{26} \mathrm{Al}$ (dashed) and ${ }^{10} \mathrm{Be}$ (dotted) through an interglacial of $15 \mathrm{ka}$ (exposure) and then a glacial period of 85 ka (burial). A range of subglacial erosion scenarios are shown during burial, from $0 \mathrm{~cm}$

$\mathrm{ka}^{-1}$ (green) to $5 \mathrm{~cm} \mathrm{ka}^{-1}$ (blue) in $0.5 \mathrm{~cm} \mathrm{ka}^{-1}$ intervals. Cosmogenic inheritance occurs where the nuclide concentrations do not decay to $\sim$ zero by the end of the glacial period. For both ${ }^{26} \mathrm{Al}$ and ${ }^{10} \mathrm{Be}$, nuclides decay to $<1 \%$ of their pre-burial concentrations (grey lines) after $\sim 77 \mathrm{ka}$ of burial with $3.5 \mathrm{~cm} \mathrm{ka}^{-1}$ of erosion and after $\sim 54 \mathrm{ka}$ of burial with $5 \mathrm{~cm} \mathrm{ka}^{-1}$ of erosion. However, cosmogenic inheritance results from either less erosion and/or a shorter burial period. The $1 \%$ cut-off is partly arbitrary, approximately representing the detectability of inheritance from concentration measurements.

and post-depositional movement and surface erosion of the erratic boulder at Halfway Nunatak could have artificially lowered the sample exposure age. However, the boulder was perched on bedrock and appeared unweathered, and is therefore considered a reliable exposure age. The dates of grounded ice retreat near Ross Island and at Hatherton Glacier are derived from radiocarbon dating, which suffers from an uncertain reservoir effect (corrections in the Ross Sea range from 1.1 ka to >1.4 ka) (Hall et al., 2010; Anderson et al., 2014) and may therefore be unreliable deglaciation estimates. Additionally, surface-exposure dating is dependent on the nuclide production rate, which is relatively unconstrained in Antarctica (Chapter 3) and may therefore be a source of age uncertainty. However, surface-exposure dating at Hatherton Glacier records deglaciation at $\sim 6.5 \pm 1.2$ ka, broadly in agreement with the radiocarbon chronology (Joy et al., 2014), suggesting minimal offset between dating techniques in this region. Alternatively, glaciological 
effects may be responsible for the lag between the timing of grounded ice retreat and final thinning at Halfway Nunatak. Surface lowering is dependent on the speed of dynamic adjustment and upstream propagation of thinning (Howat et al., 2007; Nick et al., 2009) and, in Transantarctic Mountain outlets, may account for a 1,000-year delay (Anderson et al., 2004). Furthermore, the local grounding-line possibly retreated slowly up the narrow Skelton Inlet, influenced by enhanced lateral drag from reduced valley width (Jamieson et al., 2012). It is, therefore, most likely that the lag represents delayed propagation effects and local grounding-line retreat, however the true duration of this lag is masked by uncertainties in the chronologies.

In summary, only a single exposure age supports thickening of Skelton Glacier at the LGM and the final surface lowering to its modern configuration. Despite poor direct age constraint, all sampled nunataks demonstrate past burial, predominantly by non-erosive ice, consistent with the simulated LGM profile and basal conditions of Skelton Glacier (Chapter 4). Together, this evidence suggests that thickening occurred in the lower reaches of Skelton Glacier at the LGM from buttressing at the terminus, and that subsequent surface lowering persisted until the mid-Holocene. Final thinning may have lagged the downstream retreat of grounded ice in the Ross Embayment, but dating uncertainties preclude further assessment.

\subsubsection{Long-term nuclide production rate higher than today}

Nuclide concentrations suggest that the nunataks at Skelton Glacier experienced a production rate higher, on average, in the past (SLHL; Balco et al., 2008). This is shown from samples with high ${ }^{10} \mathrm{Be}$ concentrations but also significantly low ${ }^{26} \mathrm{Al} /{ }^{10} \mathrm{Be}$ ratios (Figure 5.5A). In theory, glacial erratics could have been exposed upstream at a higher elevation, where the site production rate would be higher, and then redeposited at their current location. However, few ice-free areas exist upstream of Névé Nunatak that could have accommodated prior exposure of erratic clasts, and this would not account for the concentrations of a bedrock sample (CB38) at Clinker Bluff that must have accumulated nuclides in situ. To account for the nuclide concentrations of $\mathrm{CB} 38$, the production rate of ${ }^{10} \mathrm{Be}$, and therefore ${ }^{26} \mathrm{Al}$, must have been at least twice that of today on average over the period of complex exposure (Figure 5.5B).

It can be assumed with confidence that this represents a higher production rate because: 1) as mentioned, these concentrations are recorded in a bedrock sample that would have accumulated nuclides in situ (i.e. not from a higher elevation site upstream); 2) the production ratio between ${ }^{10} \mathrm{Be}$ and ${ }^{26} \mathrm{Al}$ would have remained constant through time, influ- 
enced by the same cosmic ray flux and production variables in quartz; and 3) nuclide concentrations were determined accurately, supported by indistinguishable inter-laboratory measurements. In order to explain higher production rates at these sites in the past, timedependent influences on production need to be assessed.

Firstly, while the bedrock sample could not have experienced a previously higher production rate upstream, the nunatak likely underwent changes in altitude from isostatic uplift. During ice cover (e.g. glacial periods), the crust is isostatically depressed; during deglaciation (e.g. interglacials), glacio-isostatic uplift occurs from unloading of the crust. Following this relationship, the nunataks may have been exposed to cosmic radiation at higher altitudes in the past (i.e. with higher-than-present production rates), if ice volume in the vicinity of Skelton Glacier was substantially less than today. To account for an apparent double production rate in the past, and assuming $\sim 0.12 \%$ change in site production rate per metre change in elevation (Figure 3.9), the nunataks would have to be $\sim 800$ $m$ higher than today. However, it is likely in the Transantarctic Mountains that long-term isostatic tectonic uplift, in response to valley incision (Stern et al., 2005), caused an opposing effect of increasing nunatak elevations and site production rates towards present. Therefore, while isostatic uplift would have influenced the site production rates, it is unlikely that this can explain a much higher production rate in the past.

Secondly, time-dependent effects on the production rate can also be related to the amount of cosmic radiation reaching the Earth's surface. Variations in surface flux result from changes in: 1) palaeo-intensity of primary radiation; 2) planetary magnetic field and solar modulation of galactic cosmic rays; 3 ) geomagnetic field effect from variations in dipole intensity and position; 4) atmospheric shielding (Gosse and Phillips, 2001). The largest, most identifiable and best understood effects are from the geomagnetic field strength, which is currently unusually high, producing production rates lower on average than in the past (Balco et al., 2008). Geomagnetic field variations are constrained over the last $\sim 2$ Ma from archeomagnetic and palaeomagnetic records (e.g. Guyodo and Valet, 1999; Laj et al., 2004; Korte and Constable, 2005; Ziegler et al., 2011). Various production rate scaling schemes attempt to account for these temporal variations (Dunai, 2000; Desilets and Zreda, 2003; Desilets et al., 2006; Balco et al., 2008; Lifton et al., 2005, 2014) as a function of cutoff-rigidity (Chapter 3 ). Production rates resulting from such scaling schemes differ on average by $\sim 0.4 \%$ from those without time-dependent magnetic field effects (Balco et al., 2008), with a larger difference for low-latitude sites where higher cutoff-rigidities have greater control on production rate variations (e.g. Desilets and Zreda, 2003; Pigati and Lifton, 2004). Based on this current understanding of geomagnetic field variations and related scaling of production rates through time, Skelton Glacier should have experienced very minor changes in production rate in the past due to 
its high latitude. However, cosmogenic nuclide concentrations have been shown to vary at higher magnitudes than recorded in these palaeomagnetic reconstructions (Ménabréaz et al., 2011), suggesting that current production rates may underestimate past variations in nuclide production.

In summary, the nuclide concentrations recorded at Skelton Glacier require a long-term production rate at least double that of today on average. This is not easily explained by the current knowledge of time-dependent nuclide production. Our inadequate understanding is highlighted by suggestions that scaling schemes, which incorporate past variations in the magnetic field, may not sufficiently capture past changes in production rate. This is an expanding field of research, with recent work seeking to constrain nuclide-specific production scaling factors (e.g. Argento et al., 2013; Lifton et al., 2014) and better understand atmospheric effects that likely influenced production rates under past climates (Lifton, 2014). Reliable application of surface-exposure dating over long timescales, as recorded here, requires improved constraints and modelling of time-dependent production rate variability.

\subsubsection{Burial-exposure history since the Miocene}

Multiple-isotope analysis revealed significant cosmogenic inheritance in samples at Skelton Glacier, indicative of a complex exposure history of the nunataks with intermittent burial by non-erosive ice. This supports model-derived ice surface profiles of Skelton Glacier under Quaternary and Pliocene climates (Chapter 4); overriding by cold-based ice was predicted during glacial periods, while nunatak exposure was predicted during intervening interglacials when the ice surface was lower or similar to present. Fluctuations between these glacial and interglacial states would have, therefore, subjected the nunataks to multiple periods of burial and exposure. Surface-exposure dating suggests, if an average long-term production rate twice that of today is assumed, that such glacial-interglacial cycles would need to account for as much as $\sim 10$ Ma of cumulative exposure and $>2.5$ Ma of burial.

Further investigation of the burial-exposure history recorded at Skelton Glacier can be achieved with the modelling of nuclide concentrations through time (e.g. Briner et al., 2006; Lilly et al., 2010). Here, a burial-exposure model has been developed to investigate the complex histories at these nunataks and to test how long Skelton Glacier has experienced glacial-interglacial fluctuations (Appendix C). This model calculates exposure and burial as stated in Equations 3.1-3.3, using the decay constants of $5.10 \times 10^{-7} \mathrm{yr}$ for ${ }^{10} \mathrm{Be}$ (Nishiizumi et al., 2007) and $9.83 \times 10^{-7} \mathrm{yr}$ for ${ }^{26} \mathrm{Al}$ (Nishiizumi, 2004; Balco et al., 
2008), an attenuation length of $160 \mathrm{~g} \mathrm{~cm}^{2}$ (Balco et al., 2008) and a rock density as per the measured samples (e.g. $2.2 \mathrm{~g} \mathrm{~cm}^{3}$ ). Production is calculated for spallation using a doubled SLHL production rate $\left({ }^{10} \mathrm{Be}, 4.49\right.$ atoms $\mathrm{g} \mathrm{a}^{-1}$ (x2); Balco et al., 2008) over this time. Production by muons is small ( $\sim 1.5-2 \%$ that of spallation; Tables 5.3 and 5.4$)$ as a result of high energies and only minor nuclide interaction in the upper rock surface, and is therefore considered negligible (e.g. Briner et al., 2006; Lilly et al., 2010). The resulting nuclide concentrations are then normalised to SLHL (x2) and plotted with measured concentrations (normalised by the site-specific production rate, $\mathrm{x} 2$ ) for comparison.

In order to model the long-term history of Skelton Glacier, the frequency and duration of burial and exposure periods need to be prescribed. Downstream of Skelton Glacier, oscillations of grounded ice and open water are recorded in the ANDRILL sediment core since $5 \mathrm{Ma}$ (Figure 5.1A), reflecting orbitally-paced ice sheet fluctuations (Naish et al., 2009; Pollard and DeConto, 2009). In particular, the frequency of these fluctuations in the geological record (Naish et al., 2009) broadly match the occurrence of open water/ grounded ice cycles at the mouth of Skelton Glacier, derived from an orbitally-driven ice sheet model (Pollard and DeConto, 2009) (Figure 5.7A). Assuming that grounded ice at the mouth of Skelton Glacier would buttress ice flow and cause upstream thickening, as previously discussed, the modelled grounding events at the terminus can be used as a proxy for sample burial since the Pliocene.

Burial and exposure is modelled over $5 \mathrm{Ma}$ using the orbitally-paced ice sheet fluctuations, under various subglacial erosion and subaerial weathering scenarios (Figure 5.7B). For zero erosion and weathering, final simulated nuclide concentrations equate to $\sim 600$ ka of cumulative exposure and $\sim 1.8 \mathrm{Ma}$ of burial, which fails to reproduce the concentrations measured in the samples. Under scenarios of increased weathering $\left(0.16 \mathrm{~mm} \mathrm{ka}^{-1}\right.$ and $0.7 \mathrm{~mm} \mathrm{ka}^{-1}$; Fink et al., 2006; Ivy-Ochs et al., 1995), concentrations of both ${ }^{26} \mathrm{Al}$ and ${ }^{10} \mathrm{Be}$ are lost more readily, evolving away from the measured concentrations. The same is true for subglacial erosion, where greater erosion leads to smaller nuclide concentrations, acting to reduce the cumulative burial and exposure time. It is, therefore, demonstrated that the complex exposure history at Skelton Glacier requires ice sheet fluctuations over longer than $5 \mathrm{Ma}$, combined with minimal erosion.

The burial-exposure model is extended back to $15 \mathrm{Ma}$ in an attempt to explain the complex sample histories. This predates the geological and ice sheet model constraints downstream of Skelton Glacier, and therefore additional drivers of burial and exposure are required from $15 \mathrm{Ma}$ to $5 \mathrm{Ma}$. Ice sheet fluctuations in the western Ross Sea are recognised to occur with variations in obliquity (in 41-ka cycles) during the Early Pliocene (Naish et al., 2009) and eccentricity (in 125-ka cycles) during the Early Miocene (Naish et al., 2001). An orbital control is also suggested during the Mid-Late Miocene ( $\sim 15-5 \mathrm{Ma})$, however 


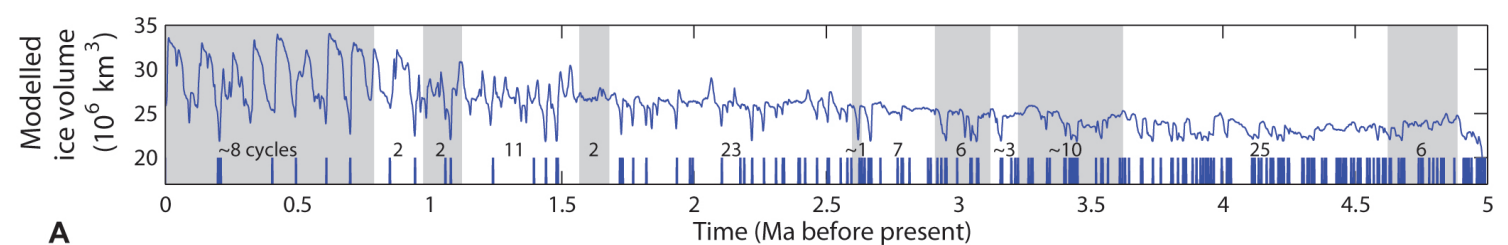

A

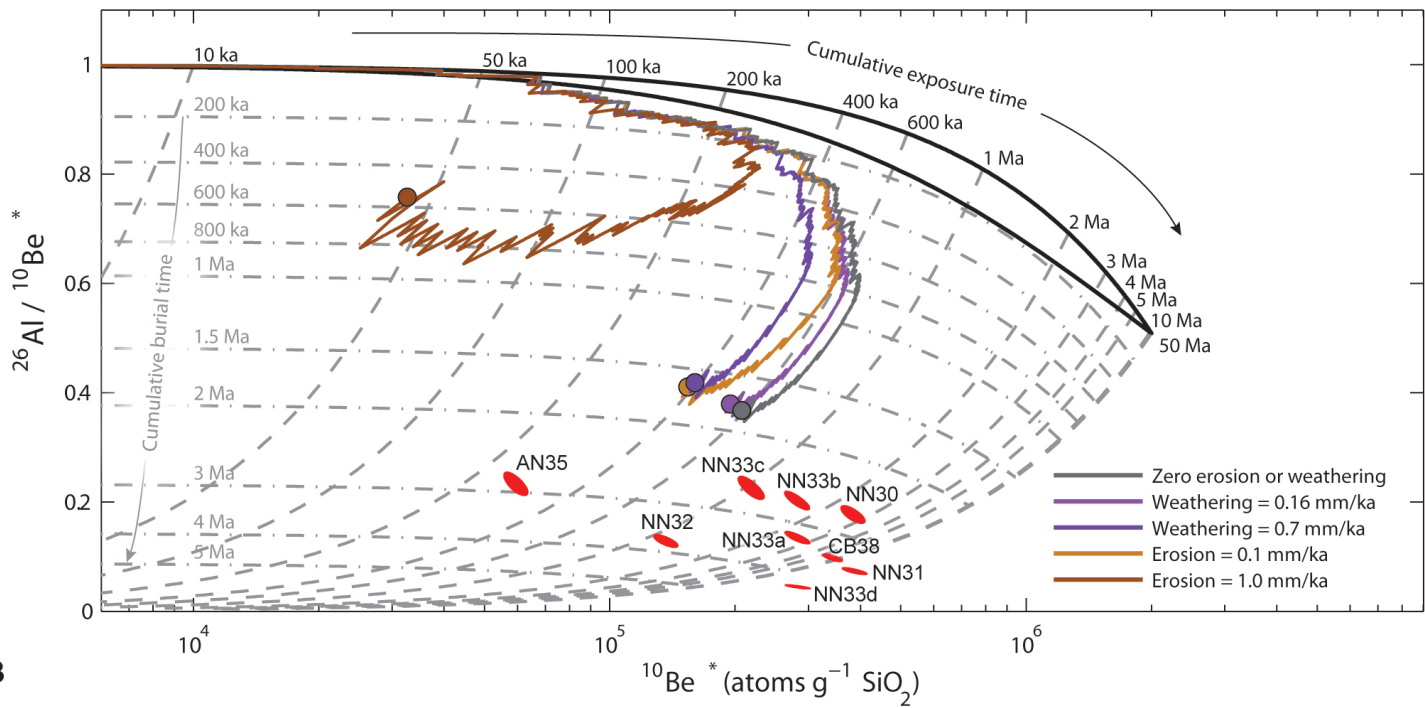

Figure 5.7 Modelled burial-exposure history over the last 5 Ma. A) Modelled ice volume (blue line) and corresponding periods of ungrounded ice (blue bars) at the mouth of Skelton Glacier (Figure 5.1A) (Pollard and DeConto, 2009). It shows orbitally-paced ice sheet fluctuations at periodicities of $41-\mathrm{ka}$ from $5 \mathrm{Ma}$ to $\sim 800 \mathrm{ka}$ and then of $100-\mathrm{ka}$ until present. Ice sheet fluctuations recorded at AND-1B (Figure 5.1A) (Naish et al., 2009) are shown here in grey with the identified number of glacial cycles, with inferred cycles shown with a white background where the glacial history was not preserved. These modelled and empirically-derived ice sheet fluctuations broadly agree, despite the model slightly underestimating the frequency and duration of interglacial periods. B) Burial-exposure history modelled for a selection of erosion and weathering scenarios, forced with the 5 Ma-to-present glacial history in A, where periods of ungrounded ice equate with exposure at the nunataks. Final concentrations (circles) can be compared to measured sample concentrations at Skelton Glacier (Figure 5.5; red ovals). Ice sheet fluctuations over the last $5 \mathrm{Ma}$, with zero subglacial erosion during burial and subaerial weathering during exposure, provide insufficient cumulative burial or exposure time to explain the measured concentrations. Increased erosion and/or weathering only acts to reduce both the final burial and exposure time.

it is unknown whether this is obliquity or eccentricity dominated (Wilson et al., 2012). Therefore, variations in both obliquity and eccentricity are used here to extend the burialexposure model back to $15 \mathrm{Ma}$. Periods of burial and exposure are determined with a constant threshold value, below which burial is modelled and above which exposure is modelled (Figure 5.8A). This threshold is adjusted to explore the sensitivity of nuclide concentrations to these forcing parameters over 15-5 Ma.

Nuclide concentrations are modelled from $15 \mathrm{Ma}$ to present under various burial-exposure scenarios (Figure 5.8). Firstly, it is shown that the length of burial and exposure periods, 

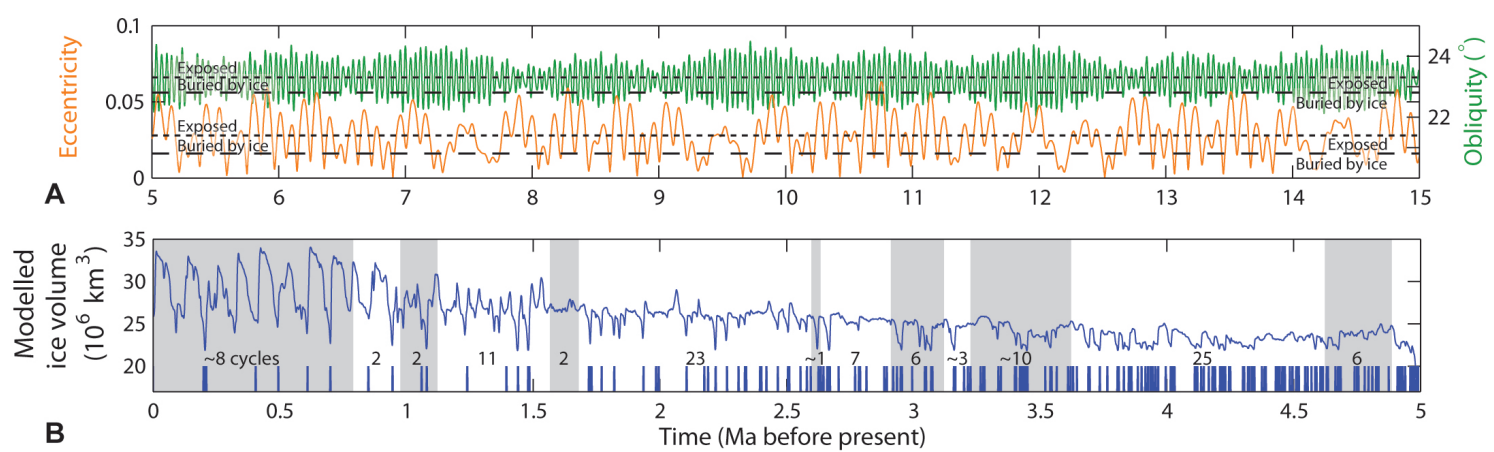

B

Time (Ma before present)

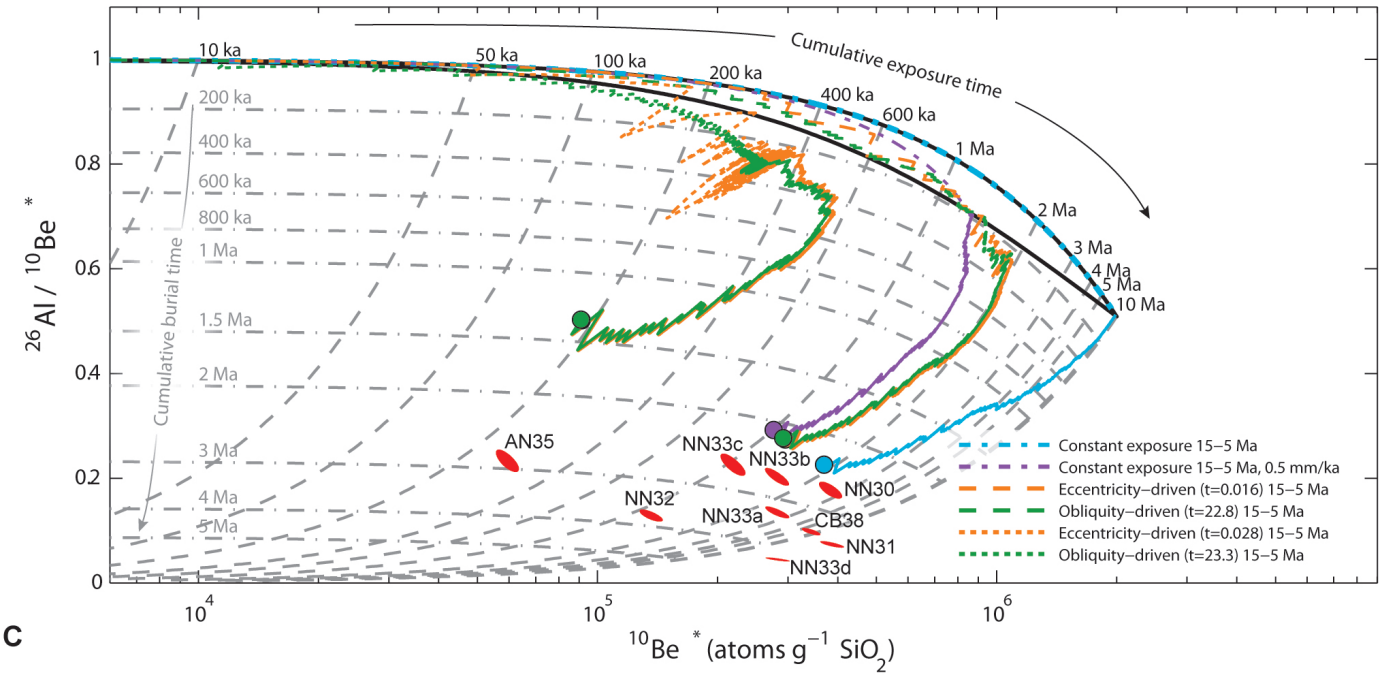

Figure 5.8 Modelled burial-exposure history over the last 15 Ma. A) Orbital forcing used in model from $15 \mathrm{Ma}$ to $5 \mathrm{Ma}$. Ice sheet fluctuations are inferred from variations in eccentricity and obliquity, where a constant threshold is used to delimit nunatak exposure versus nunatak burial. These are shown as dotted and dashed lines, which

related to evolving concentrations in C. B) Modelled ice volume and episodes of ungrounded ice (as in Figure 5.7), using to force burial-exposure model from $5 \mathrm{Ma}$ to present. C) Burial-exposure history for a selection of scenarios from 15-5 Ma, followed by the same history for 5-0 Ma. Measured sample concentrations at Névé Nunatak and Clinker Bluff are most closely replicated with constant exposure 15-5 Ma, both with zero subaerial weathering and $0.5 \mathrm{~mm} / \mathrm{ka}$ of weathering. Similar final concentrations result from orbitally-paced burial-exposure history over this time. Interestingly, nuclide concentrations show contrasting magnitudes and frequencies of burial/exposure whether eccentricity or obliquity driven, however these differences are reduced with a common

forcing after $5 \mathrm{Ma}$, resulting in near-identical final concentrations. Using higher thresholds $(\mathrm{t})$, and therefore greater burial time per cycle, concentrations evolve towards

AN35 but without sufficient cumulative burial time. This modelling highlights that greater burial is required during the last $5 \mathrm{Ma}$ to explain the measured sample concentrations.

and therefore evolution of nuclide concentrations, varies greatly depending on the orbital forcing used. However, any differences between the obliquity and eccentricity driven concentrations are greatly reduced after $5 \mathrm{Ma}$, when the common forcing is applied. Despite very different nuclide histories from 15-5 Ma, final nuclide concentrations can be near-identical, indicating negligible effect from the type of orbital forcing used. Secondly, sample concentrations at Névé Nunatak and Clinker Bluff can be most closely replicated 
if the nunataks were constantly exposed at 15-5 Ma (with subaerial weathering between $0 \mathrm{~mm} \mathrm{ka}{ }^{-1}$ and $0.5 \mathrm{~mm} \mathrm{ka}^{-1}$ ), or if the nunataks experienced repeated burial and exposure at obliquity or eccentricity timescales at $15-5 \mathrm{Ma}$ (using orbital threshold values of $\mathrm{t}=22.8^{\circ}$ and $\mathrm{t}=0.016$, respectively), prior to Pliocene-to-present ice sheet fluctuations. By increasing the amount of burial per cycle for the period $15-5 \mathrm{Ma}$ (using $\mathrm{t}=23.3^{\circ}$ and $\mathrm{t}=0.028$ ), nuclide concentrations evolve slightly towards AN35 at the unnamed nunatak, but the simulated cumulative burial time is less than that suggested by the measured concentrations by $\sim 1.5 \mathrm{Ma}$. Importantly, irrespective of the burial-exposure history between $15 \mathrm{Ma}$ and $5 \mathrm{Ma}$, insufficient minimum cumulative burial time is generated from the partconstrained burial-exposure history at 5-0 Ma. This analysis, therefore, suggests that Skelton Glacier possibly experienced ice sheet fluctuations at orbital timescales since at least $15 \mathrm{Ma}$, however the measured sample concentrations indicate $>0.5 \mathrm{Ma}$ additional burial time that is unaccounted for.

The offset between measured sample concentrations at Skelton Glacier and the simulated concentrations could be explained by a number of factors not included in the burialexposure model. Since the initiation of ice sheets in Antarctica, glaciers have incised progressively deeper outlet systems (e.g. Stern et al., 2005; Jamieson et al., 2010), facilitating long-term lowering of the ice sheet surface (Lilly et al., 2010). At Skelton Glacier, erosion was modelled in the central trunk during Pliocene and Quaternary glacials and interglacials (Chapter 4), suggesting the possibility of long-term ice surface lowering. This would, however, lead to progressively more exposure at the nunataks instead of greater burial time, as suggested by the sample concentrations. Additional burial time could also be explained by large, non-cyclic events at the nunataks in the last $5 \mathrm{Ma}$. A glacial episode with a duration of 0.5-1 Ma is unlikely, and the mouth of Skelton Glacier likely experienced more open water conditions than full glacial conditions during the Pliocene (Naish et al., 2009). Alternatively, these nunataks may have become covered by thick till, followed by slow deflation. Significant till production may have been possible during a particularly substantial interglacial (Chapter 4; DeConto et al., 2012), with deposition from overriding ice during a subsequent glacial episode. This would act to reduce the ${ }^{26} \mathrm{Al} /{ }^{10} \mathrm{Be}$ ratio and, therefore, increase the cumulative burial time, irrespective of ice sheet fluctuations over the last $5 \mathrm{Ma}$. Subsequent deflation of till would then re-exposed the nunatak, but potentially at some samples before others.

Further contributions to this apparent burial time offset may be associated with uncertainties in the nuclide concentration calculations. As discussed, long-term variations in the production rate are relatively unconstrained, especially prior to $\sim 800 \mathrm{ka}$. The evolution of nuclide concentrations could, therefore, have differed slightly to what is modelled here, depending on how these variations in production rate coincided with cycles of burial and exposure. Additionally, nuclide production was only calculated by spallation and, al- 
though considered small, the contribution from muons is not accounted for. Lastly, calculations here use values of attenuation length and rock density that are assumed. Moderate differences in these parameters can affect the amount of exposure and burial time (Figure 3.4), and may partly explain imperfect simulation of the measured sample concentrations.

In summary, the complex history of samples at Névé Nunatak, the unnamed nunatak and Clinker Bluff likely represents exposure and burial since at least the Mid-Miocene. Burial-exposure modelling suggests that these nunataks could have been overridden by a thickened Skelton Glacier that fluctuated on orbital timescales during this time, but continuous pre-Pliocene exposure cannot be ruled out. Nearby evidence from the East Antarctic plateau edge indicates near-continuous nunatak exposure since the Early Pliocene (Balco et al., 2014). Together with the data from Skelton Glacier, this suggests that Transantarctic Mountain outlet glaciers varied in size in response to fluctuating grounded ice in the Ross Embayment, while the upstream East Antarctic Ice Sheet remained relatively stable since at least the Pliocene.

\subsection{Conclusions}

The past configurations of Skelton Glacier have been investigated with surface-exposure dating. Its lower reaches were likely thicker than today at the LGM, supporting regional evidence that grounded ice in the Ross Sea acted to buttress the flow of Transantarctic Mountain outlet glaciers. Nunataks adjacent to the main flow path of Skelton Glacier experienced negligible erosion under probable cold-based ice, consistent with glacier modelling experiments. Samples at the nunataks record a complex history that can be explained by fluctuations of Skelton Glacier at orbital timescales since the Early Pliocene, and possibly Mid-Miocene. Over this time, sample concentrations indicate that nuclide production rates were, on average, at least twice those of today, highlighting the need to better constrain long-term variations in past production rates if surface-exposure dating is to be reliably applied on these timescales. 


\section{Chapter 6}

\section{Long-term palaeo-dynamics of Mackay Glacier inferred from preserved and modified geomorphology}

\subsection{Introduction}

This study aims to identify the past configurations and behaviour of Transantarctic Mountain outlet glaciers under different climatic settings by investigating a sequence of geomorphological signatures. Past outlet glacier behaviour can provide an analogue for both modern changes in basal conditions that are currently unattainable with satellite and ground measurements, and long-term responses of ice sheets to changing boundary conditions. Numerical ice sheet models are able to quantify past ice dynamics (e.g. Denton and Hughes, 2002; Huybrechts, 2002; Pollard and DeConto, 2009; De Boer et al., 2013; Golledge et al., 2013), and in doing so, improve the reliability of simulations of future ice sheet scenarios. These models typically use geologic-geomorphic data as temporal and spatial constraints on past ice sheet geometries and dynamics, but are limited by the quality and availability of data.

Glacial geologic-geomorphic features can be found beyond the present-day ice sheet margins. On the continental shelf, previous maximum extents of grounded ice are determined by seismic surveys of glacial deposits such as grounding-zone wedges (e.g. Bart et al., 2000; Shipp et al., 1999), while styles of advance and retreat can be inferred from the type and distribution of this offshore geomorphology (e.g. Greenwood et al., 2012; Klages et al., 2014). Terrestrial records are limited to the few ice-free areas at nunataks and the fringes of the present-day glaciers. In the Transantarctic Mountains, deeply incised valleys of outlet glaciers are flanked by aerially scoured surfaces that emanate from an ancient, more erosive glacial setting (e.g. Sugden and Denton, 2004). Various terrestrial deposits and formations have been studied in combination with dating techniques (e.g. surface exposure, tephra and radiocarbon dating) to determine the presence or absence of ice sheet activity at different times. These include weathering boundaries (e.g. Di Nicola et al., 2012), meltwater features (e.g. Lewis et al., 2006), soil profiles (Dickinson et al., 2012), shorelines (e.g. Hall et al., 2004) and glacial deposits. Surficial glacial drift deposits and moraines have been used to delimit episodes of more extensive ice cover in this region, whereby the differing lithological and soil compositions enabled relative age control and correlation between sites (e.g. Bockheim et al., 1989; Denton et al., 1989; Lewis et al., 2007; Bromley et al., 2012). Evidence of cold-based ice, that can either erode and redeposit or cover and preserve existing geomorphology (Atkins et al., 2002; 
Lloyd-Davies et al., 2009; Atkins, 2013), implies that these deposits may not be recording sequential glacial advances, but instead may represent a mixture of formation and modification events. Furthermore, the use of drift limits and moraine positions as indicators of past ice sheet thicknesses or extents may be misleading if they represent a previous glacial thermal boundary (warm- to cold-based ice) (e.g. Miller et al., 2002), as suggested by modelling of Transantarctic Mountain outlet glaciers (Golledge et al., 2013). If appropriately assessed, glacial geomorphology has the potential to provide a constraint on past glacier dynamics in addition to ice sheet geometry. Here, I attempt to improve our understanding of the past dynamics of these outlet glaciers by focusing on Mackay Glacier, an outlet glacier that has been in existence since before inception of an East Antarctic Ice Sheet (EAIS) (Sugden and Denton, 2004).

Since the end of the Eocene when the Southern Hemisphere experienced a major cooling, global sea level records indicate the formation and fluctuations of large Antarctic ice sheets between $34 \mathrm{Ma}$ and $14 \mathrm{Ma}$ (Shackleton et al., 1975; Miller et al., 2005). Such fluctuations occurred at orbital timescales, possibly between modern and fully glacial states (Naish et al., 2001), driven by declining $\mathrm{CO}_{2}$ concentrations (e.g. DeConto and Pollard, 2003; Pagani et al., 2005; Huber and Nof, 2006), rapid drops in temperature (Liu et al., 2009) and increased moisture availability from the Southern Ocean (Kennett, 1977). A sharp transition from obliquity to eccentricity paced orbital forcing during the mid-Miocene led to major ice sheet expansion in Antarctica (Holbourn et al., 2005) and high amplitude advance and collapse cycles continued through the Pliocene (Naish et al., 2009; Pollard and DeConto, 2009), at least in the case of the West Antarctic Ice Sheet (WAIS).

The last cycle of expansive ice sheets peaked at the Last Glacial Maximum (LGM). This event is relatively well recorded in the western Ross Sea, with the LGM grounding-line located near to the continental shelf edge (Anderson et al., 2014). Grounded ice in the Ross Sea was sourced from both WAIS via ice streams and EAIS through the Transantarctic Mountains (Licht et al., 2005), with a sloped surface from the interior to the coastal margin (Golledge et al., 2012). Geomorphic interpretations support this ice surface gradient along the south-north front of the Transantarctic Mountains (Figure 6.1A). Based primarily on the elevation of Ross Sea Drift, the LGM ice surface sloped from Minna Bluff and the east side of Ross Island ( $\sim 640$ and $\sim 710 \mathrm{~m}$ asl respectively; Denton and Marchant, 2000) past the mouth of Mackay Glacier to Terra Nova Bay ( 300-400 m asl; Orombelli et al., 1990). A modelled ice sheet geometry at the LGM agrees with surface reconstructions near Mackay Glacier, suggesting an ice elevation of $\sim 600-700 \mathrm{~m}$ asl at the present-day grounding-line (Denton and Hughes, 2000; Golledge et al., 2013). 

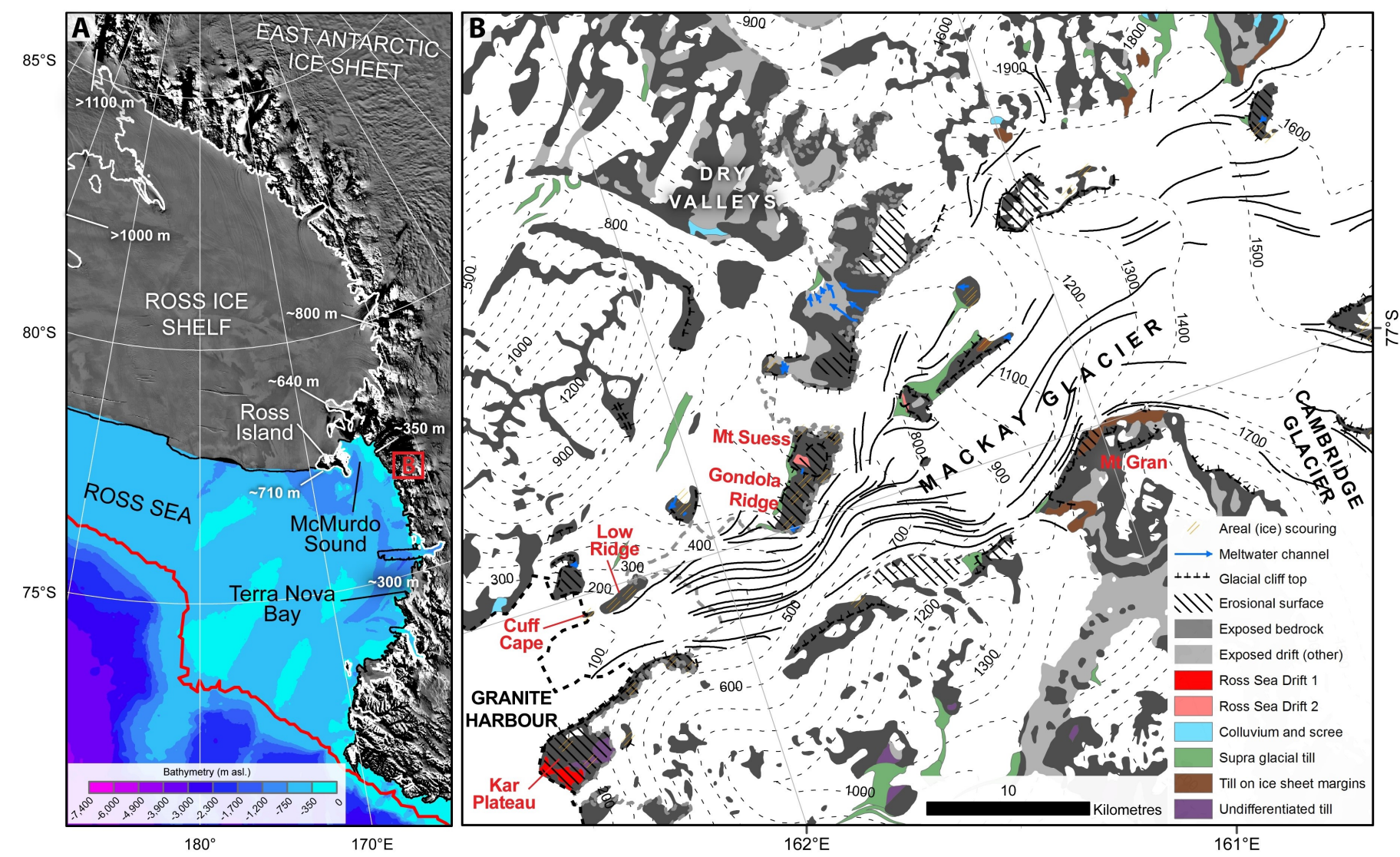

Figure 6.1 Location of Mackay Glacier and study sites. A) View along the Transantarctic Mountains and western Ross Embayment, with labelled constraints on LGM ice surface elevations (in m asl) and modelled LGM extent of grounded ice (red line; Golledge et al., 2013). B) Study sites at Mackay Glacier and coarse-scale geomorphology of the area. Shown are interpreted erosional landscape features (Sugden and Denton, 2004) and surficial deposits (Cox et al., 2012), with mapped glacier surface lineations to denote flow direction within the catchment. The present-day ice sheet grounding-line is shown as a solid white line over MOA ice surface contrast imagery in A and a dashed grey line in B, while the coast and ice shelf edge is shown as a solid black line in A and dashed black line in B. Ice surface elevations from BEDMAP2 (Fretwell et al., 2013) are contoured every $100 \mathrm{~m}$ asl in B. 
Reduced precipitation and possibly enhanced katabatic-driven ablation during glacial periods in the area of the Dry Valleys (McCrae, 1984) might have influenced past ice-flow patterns, potentially causing westward flow of grounded ice in McMurdo Sound (Denton and Hughes, 2000; Golledge et al., 2013). These local climatic factors are also hypothesised to explain the limited expansion and likely reduced size of local glaciers in the Dry Valleys at the LGM (Denton and Hughes, 2000; Denton and Marchant, 2000; Hall et al., 2000). Considering the location of Mackay Glacier adjacent to the Dry Valleys, and the understanding that the EAIS interior was starved of accumulation during glacials (Steig et al., 2000), Mackay Glacier may have been thinner than today at that time. Alternatively, it could have thickened in response to lowering sea level and/or buttressing effects from grounded ice in the Ross Sea, as documented for other Transantarctic Mountain outlet glaciers (e.g. Bockheim et al., 1989).

Today, observations reveal the close relationships between climate and ice sheet volume and dynamics (Hanna et al., 2013), and these have likely held true since the inception of glaciers in Antarctica. Initial conditions under high $\mathrm{CO}_{2}$ concentrations were favourable for extensive warm-based ice. Near Mackay Glacier, mean summer air temperatures were above $5^{\circ} \mathrm{C}$ during the Oligocene to Early Miocene (Roberts et al., 2003; Prebble et al., 2006). Environmental conditions then changed at the mid-Miocene transition, when surface waters in the south-west Pacific cooled by 6-7 ${ }^{\circ} \mathrm{C}$ (Shevenell et al., 2004). Subsequent glacial episodes experienced uniformly cold ice sheet surface temperatures $\left(<-10^{\circ}\right.$ at the LGM), likely contributing to widespread cold-based ice in East Antarctica (Jamieson et al., 2010; Golledge et al., 2013). However, large outlet glaciers and streaming areas of WAIS could have been at, or close to, basal melting point. Faster ice-flow and therefore greater shear stress would have facilitated selective erosion in the trunk of outlet glaciers while cold-based ice occupied the flanks (Golledge et al., 2013). These conditions are therefore similar to present-day (e.g. Pattyn, 2010), and suggest alpine areas and small outlet glaciers probably did not undergo wet-based sliding during cold glacial episodes. At Mackay Glacier, the output from a recent ice sheet model (Golledge et al., 2013) indicates predominantly cold-based ice, both in its trunk and at its sides, and therefore wet-based conditions at the LGM were unlikely (Figure 6.2A and 6.2B). However, if erosion at the bed was to occur, the greatest potential was in the middle reaches of the glacier where both basal velocity and shear stress are simulated to be highest (Figure 6.2C). Glacial-geological evidence is, therefore, required to test whether Mackay Glacier was wet-based and erosive at the LGM and/or during an earlier period of glacial cycles when relatively warmer atmospheric temperatures existed.

The multiple glaciations that are hypothesised for this area have left erosional and depositional signatures, which are investigated in this chapter to identify the evolving basal and 


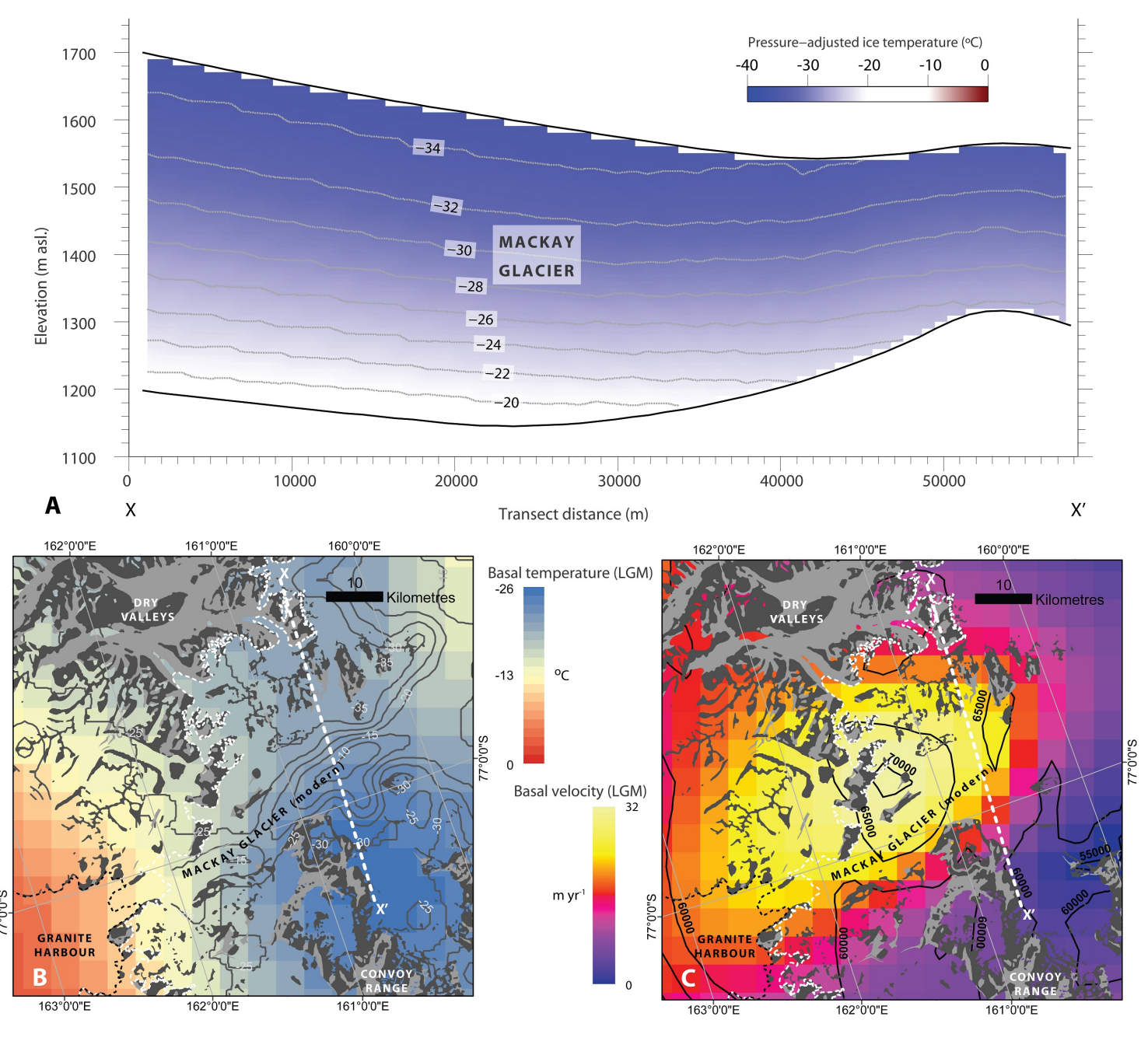

Figure 6.2 Modelled basal conditions of Mackay Glacier at the LGM. A) Vertical transect of ice temperature in the region of Mackay Glacier, simulated with a continent-scale model of an LGM ice sheet (Golledge et al., 2013). While the model (5 $\mathrm{km}$ horizontal resolution) does not capture the confined valley topography of Mackay Glacier, it indicates that the deeper trunk was likely warmer at the bed than the higher-elevation sides but that neither part of basal ice reached melting point. B) Map-plane view of modelled basal temperature (pressure-adjusted) at the LGM (Golledge et al., 2013) and present-day (Pattyn, 2010). At the LGM (coloured grid), basal ice possibly warmed to melting point beyond the Transantarctic Mountains and Granite Harbour in this area. Today (dark grey contours, every $5^{\circ} \mathrm{C}$ ), the warmest basal temperatures are indicated in the middle reaches of Mackay Glacier, but are similarly not low enough to produce melt at the bed. C) Basal velocity and shear stress at the LGM (Golledge et al., 2013). Basal erosion requires high basal velocities (coloured grid) and shear stress (black contours, every $5000 \mathrm{~Pa}$ ), both of which peak in the middle reaches of Mackay Glacier. The present-day grounding-line of the EAIS (thin, white dashed line), modern coast (thin, black dashed line) and vertical transect of A (thick, white dashed line) are located in B and C. 
flow regimes of Mackay Glacier. The following questions are addressed; 1) Was Mackay Glacier wet-based during early, pre-Quaternary ice sheet expansions? 2) How thick was Mackay Glacier at the LGM? 3) Was Mackay Glacier cold- or wet-based, non-erosive or erosive, during the LGM? 4) Was the flow of Mackay Glacier influenced by adjacent Dry Valleys glaciers and/or grounded ice in the Ross Sea?

\subsection{Geographic setting}

Mackay Glacier is an outlet of the EAIS, located immediately north of the Dry Valleys in the Transantarctic Mountains (76.9-77.2 ${ }^{\circ} \mathrm{S}, 160-162.5^{\circ} \mathrm{E}$; Figure 6.1). This landscape has evolved from fluvial planation and dissection, local temperate glaciation and then overriding by the EAIS at $\sim 15 \mathrm{Ma}$ (Sugden and Denton, 2004). The primary source of the present-day Mackay Glacier is Taylor Dome, with additional input from ice fields on the flanks of the Convoy Range and Dry Valleys. A network of meandering ice flow paths stretch $12 \mathrm{~km}$ across-valley and are over $430 \mathrm{~m}$ thick (Calkin, 1974). Measured surface velocity is $\sim 265 \mathrm{~m} \mathrm{a}^{-1}$ (1992-1998) at the grounding line (Frezzotti et al., 2000) and $\sim 250 \mathrm{~m} \mathrm{a}^{-1}$ towards the terminus (Macpherson, 1987), while velocities from satellitederived data (Rignot et al., 2011) are $3-5 \mathrm{~m} \mathrm{a}^{-1}$ at the head of the glacier to $190 \mathrm{~m} \mathrm{a}^{-1}$ at the terminus. Surface flow is heavily influenced by the basal topography, indicated by a stepped surface profile that reflects sub-glacial bedrock escarpments (Calkin, 1974). Further inference of the basal dynamics can be made from sediment observations. Till sampled at the terminus of Mackay Glacier is principally sub-glaciallly derived, but lacks fluvial deposits and therefore the presence of meltwater (Powell et al., 1996). Furthermore, sub-glacially derived englacial debris bands may signify some localised thrusting processes and have been observed adjacent to the terminus at Cuff Cape (Möller, 1995), indicative of a compressive or thermally complex regime.

\subsection{Surficial geology and geomorphology}

The ice-free flanks of Mackay Glacier consist of glaciated granitic (Granite Harbour Intrusives) and sandstone/dolerite (Beacon Supergroup) bedrock, and surficial glacial deposits. Previous studies have documented erosional surfaces, areal scour, glacial cliffs and drift deposits (Denton and Hughes, 2000; Sugden and Denton, 2004), however, such features have not been mapped in detail. This work focuses on sites that represent the uppermiddle (Mt Gran), lower-middle (Mt Suess and Gondola Ridge) and lower (Low Ridge 
and Cuff Cape) reaches of Mackay Glacier, as well as a site beyond the present-day terminus that borders the Ross Sea (Kar Plateau). Surficial geology and geomorphology was surveyed at each of these sites, with landforms and deposits mapped and respective characteristics noted (degree of weathered or glaciated material, lithologies, morphology, and relationship to Mackay Glacier). As this project aims to investigate the spatial variability of past ice dynamics inferred from the geomorphology, features were recorded with the aid of a handheld GPS ( $\pm<1 \mathrm{~m}$, horizontally) and Worldview satellite imagery $(0.5 \mathrm{~m}$ resolution).

\subsubsection{Mt Gran}

Mt Gran (2233 m asl) is an ice-free area that is located at the confluence of Cambridge and Mackay Glaciers, $\sim 26 \mathrm{~km}$ from the present-day grounding-line. On the south and southeast side of Mt Gran lie areas of relatively flat topography adjacent to the ice margin. Large snow patches, small local glaciers and highly-weathered surfaces occupy the upper slopes of Mt Gran, while glacial diamict ('drift') deposits mantle most of the bedrock below the talus-covered slopes (Figure 6.3). Here, the geomorphology has been both preserved and modified by previous glacial activity, with a signature that varies with distance from the ice margin.

A flat terrace area to the south-east of Mt Gran was mapped by Sugden and Denton (2004) as an erosional surface, cut across the Beacon Supergroup contact. Atop the bedrock lies thick cryoturbated drift (Figure 6.4A). Polygons are well-developed in places, with fine sediment brought to the surface while boulders occupy cracks at the edges, and refrozen meltwater pools intermittently occur within the deposits. The material is comprised primarily of dolerite with some sandstone input that is reflective of the local lithology. All clasts have experienced spallation, frost-shattering and chemical weathering to varying degrees. Ridge features extend across this drift deposit, sub-parallel with the glacier margin. The majority of the ridges have been heavily modified by cryoturbation with destabilised slopes and dissection by meltwater pools. At the base of Mt Gran's slopes, on both the south and south-east sides, are a set of ridges termed 'necklace moraines'. These retain continuous crest-lines and have likely been formed and possibly been mobilised by local ice and/or nivation processes. Ice-cored moraines can be found near the glacier margin and have well-developed, steep ice-proximal slopes, often with compacted till. The most ice-proximal moraine contains relatively unweathered dolerite material, which is in contrast to the other ridges that consist of heavily weathered dolerite (Figure 6.4B). 


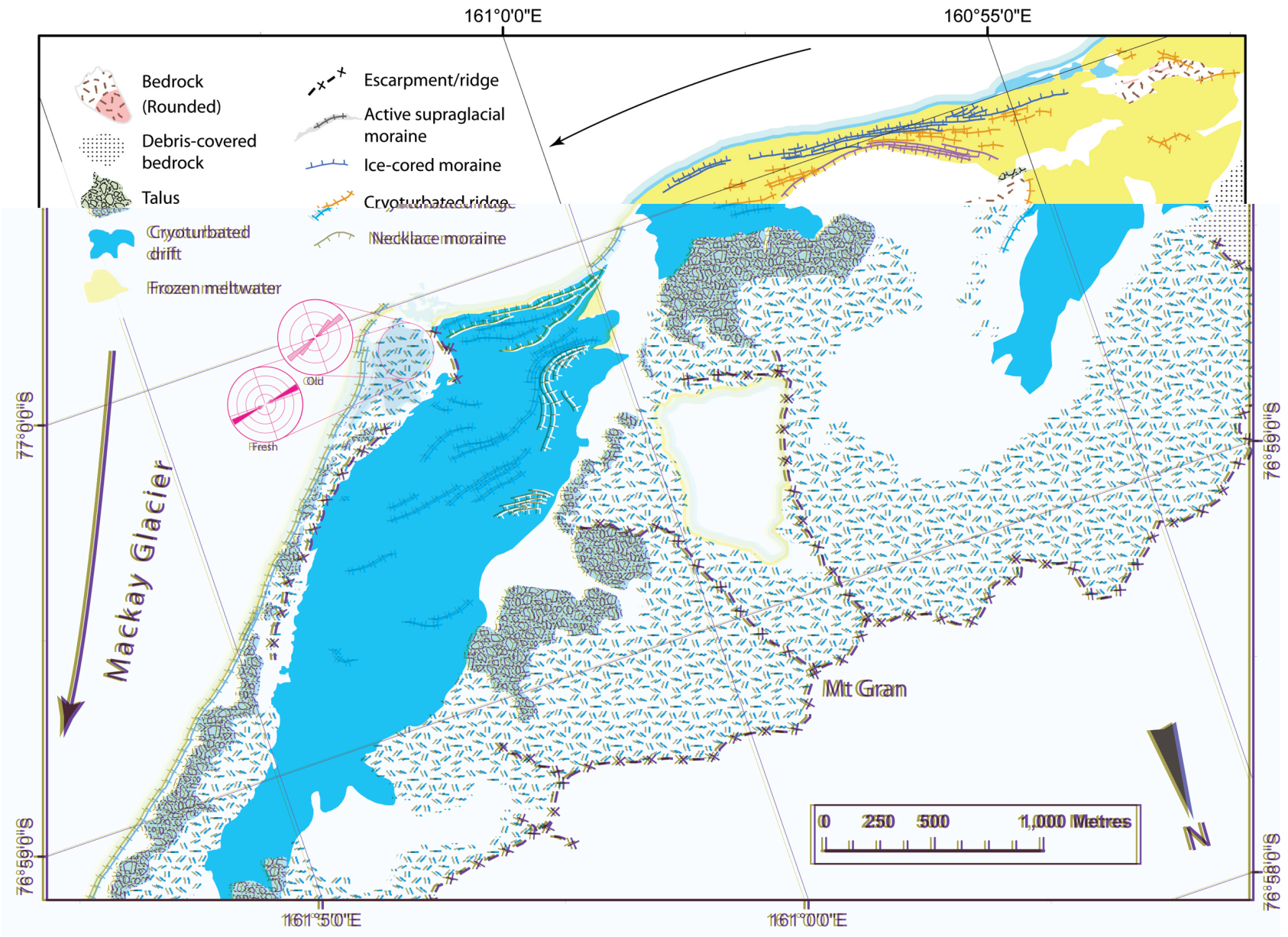

Figure 6.3 Surfical geology and geomorphology at Mt Gran, upper-middle Mackay Glacier. Cryoturbated drift and features exist in the low-lying areas adjacent to Mackay Glacier, while aerially weathered rock surfaces, year-round snow patches and a local alpine glacier occupy the higher elevations. Relatively old and fresh striae orientations are shown for the area of glacially-rounded bedrock $\left(060-070^{\circ}\right.$ and $080-090^{\circ}$ respectively), and indicate differing flow directions during different glacial events. 
Glacial erosion is most apparent as an escarpment or "glacial cliff top" (Sugden and Denton, 2004) that runs along the eastern edge of the terrace. An area of rounded dolerite bedrock lies to the south of the escarpment, and extends back from the margin of the trunk of Mackay Glacier, over a topographic bedrock high, to the edge of the drift-covered terrace. Although areas of the exposed bedrock have experienced considerable fracturing and spallation, smoothed and striated surfaces are preserved in several locations. Similar bedrock surfaces are exposed in small locations above drift deposits on the south side. Two dominant groups of striae exist; 1) narrow and deep striae that have the same oxidised surface as the surrounding bedrock, and 2) shallower, grey striae that have removed the pre-existing oxidised surface with negligible subsequent surface weathering (Figure 6.4C and 6.4D). Based on this relative age relationship, the former and latter groups are termed 'old' and 'fresh' striae, respectively. The 'old' striae are orientated 060-070 and often lie near large relict chatter-marks, while 'fresh' striae are orientated 080-090 and can be found adjacent to freshly abraded bedrock edges (Figure 6.3). Therefore, at least two glacial events are recorded with differing ice flow directions and erosional imprints. Additionally, p-forms ( $\sim 0.5 \mathrm{~m}$ deep and 5-10 $\mathrm{m}$ long) can be found on the side and below a terrace escarpment on the south-east side (Figure 6.4E), and are indicative of abrasive, debris-rich, subglacial meltwater under high pressure (Boulton, 1974; Shaw, 1988; Glasser and Bennett, 2004). Superimposed on the surface are preserved 'old' striae that suggest a similar time of formation, prior to the last episode of glacial cover. Fresh dolerite clasts that were likely deposited over this bedrock area during the last glacial episode, occur up to $\sim 1045 \mathrm{~m}$ asl, equivalent to $115 \mathrm{~m}$ above the trunk of the modern Mackay Glacier.

In summary, the ice-marginal flanks of Mt Gran record sequences of glacial erosion and deposition. An early glacial episode eroded and incised areas of a previously planed erosional surface (Sugden and Denton, 2004), forming ubiquitous rounded and striated bedrock. During this event, parts of the glacier were wet-based with substantial subglacial sediment flux and/or meltwater development. Subsequent deposition of glacial and locally-derived material formed thick drift deposits on the low gradient flanks of Cambridge and Mackay Glaciers. The last glacial episode failed to remove the drift deposit or erode the relict bedrock surfaces beyond minor surficial abrasions, but likely deposited freshly-eroded material and may have formed moraine(s) during subsequent retreat of the margin. Substantial reworking probably occurred from local glaciers and cryoturbation processes during the most recent, and possibly previous, ice-free periods. 


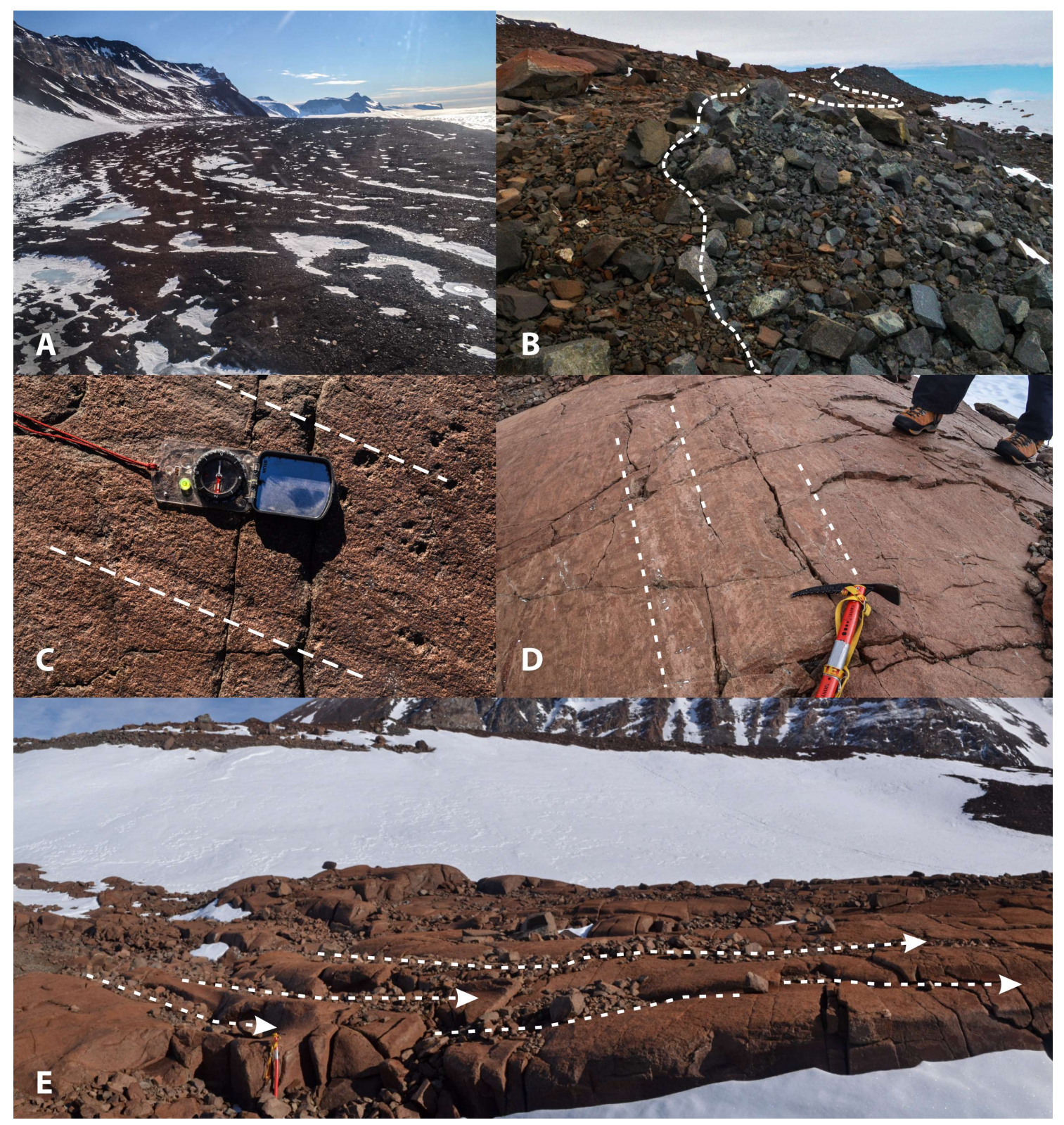

Figure 6.4 Selected representative views of geomorphic features at Mt Gran. A) View over the cryoturbated drift covered terrace, looking north-east. B) Boundary between old, weathered dolerite deposits (brown, to left) and fresher dolerite deposits (grey, to right), near the south-east ice margin. C) 'Old' striae eroded into a now oxidised and pitted dolerite bedrock surface (compass, uncorrected for magnetic north, for scale). D) 'Fresh' striae and abrasions on old, rounded and chatter-marked dolerite bedrock (ice axe for scale). E) P-forms trending downstream across the image (left to right, highlighted with dashed arrows), eroded into the old bedrock surface and part-infilled with glacial debris (ice axe for scale). 


\subsubsection{Mt Suess \& Gondola Ridge}

Mt Suess and Gondola Ridge comprise an elongate nunatak on the south side of Mackay Glacier, 7-14 km upstream of the present-day grounding line. Mt Suess (1191 m asl) is a heavily weathered basaltic dome, while the lower-lying Gondola Ridge is an expanse of rounded granite roche moutonnées (Figure 6.5). Here, both eroded and depositional landforms exist, but with limited locally-derived material.

Drift on Mt Suess was previously used to delimit an LGM ice surface, $150 \mathrm{~m}$ higher than today (Denton and Hughes, 2000). I identified drift of unknown age on Mt Suess, comprising highly weathered erratic sandstone, granite, quartzite and basaltic lithologies. A distinct ridge with a couple of smaller ridges are found on the lateral, downstream margin of the drift, but their true form is masked by cryoturbation and nivation features (Figure 6.6B). Despite this, heavily weathered sandstone erratics at $\sim 890 \mathrm{~m}$ asl $(330 \mathrm{~m}$ above the modern ice surface) and striated bedrock (Figure 6.6A) on the downstream side of Mt Suess indicate that parts of Mt Suess were glaciated sometime previously. On the upstream, western side of Mt Suess, there is a transition from freshly eroded granitic surfaces at the glacier margin, through increasingly weathered bedrock and then to regolithlike bedrock platforms below rectilinear dolerite slopes (Figure 6.7). Sandstone erratics with varied degrees of weathering can be found in high concentration across this transition up to $\sim 825 \mathrm{~m}$ asl (260 $\mathrm{m}$ above the modern glacier). These erratics were possibly deposited during the same glacial episode and therefore record ice cover up to the rectilinear slopes. If so, an erosional or weathering gradient is implied at that time, where either less bedrock erosion occurred with increased elevation at the glacier sides or more weathering occurred at higher elevations with longer prior exposure times.

Evidence of glacial erosion can be found as rounded and striated bedrock surfaces across Gondola Ridge (Figure 6.6). The orientation of these 'fresh' striae reveal the ice-flow direction during the last glacial episode. Near the north-east end of Gondola Ridge, striae indicate that ice flowed in a similar manner to today (at $070-100^{\circ}$ ), but took a more direct route downstream over the elevated bedrock. At the downstream face of Mt Suess, past ice-flow was near-perpendicular to the modern direction of Mackay Glacier (striae at $\left.110-130^{\circ}\right)$. This divergence of flow across Gondola Ridge implies previous ice-flow to the south-east that dominated any ice-flow along the east side of the nunatak.

Despite signs of erosive ice in the past, especially on lower lying areas immediately adjacent to the glacier margin, most of the bedrock surfaces are heavily spalled (Figure 6.6E and 6.6F). The presence of extensive surface weathering might be related to the coarse- 


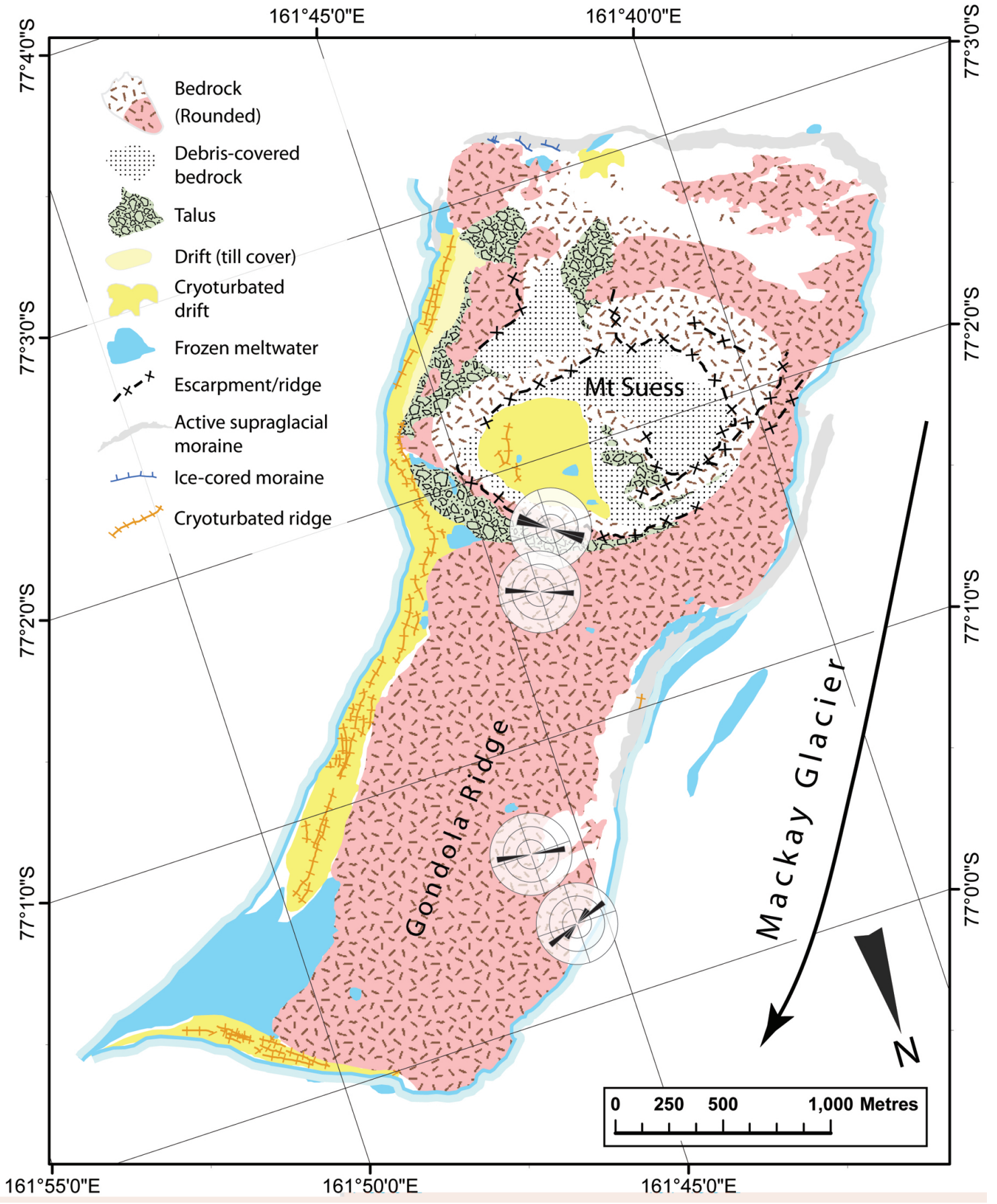

Figure 6.5 Surfical geology and geomorphology at Mt Suess and Gondola Ridge, lower-middle Mackay Glacier. The bedrock surface at this nunatak has been extensively rounded and smoothed, with surficial cover from deposits occurring in some ice-marginal and high elevation locations. Fresh striae orientations reveal the spatially variable flow directions of Mackay Glacier during the last glacial episode. 


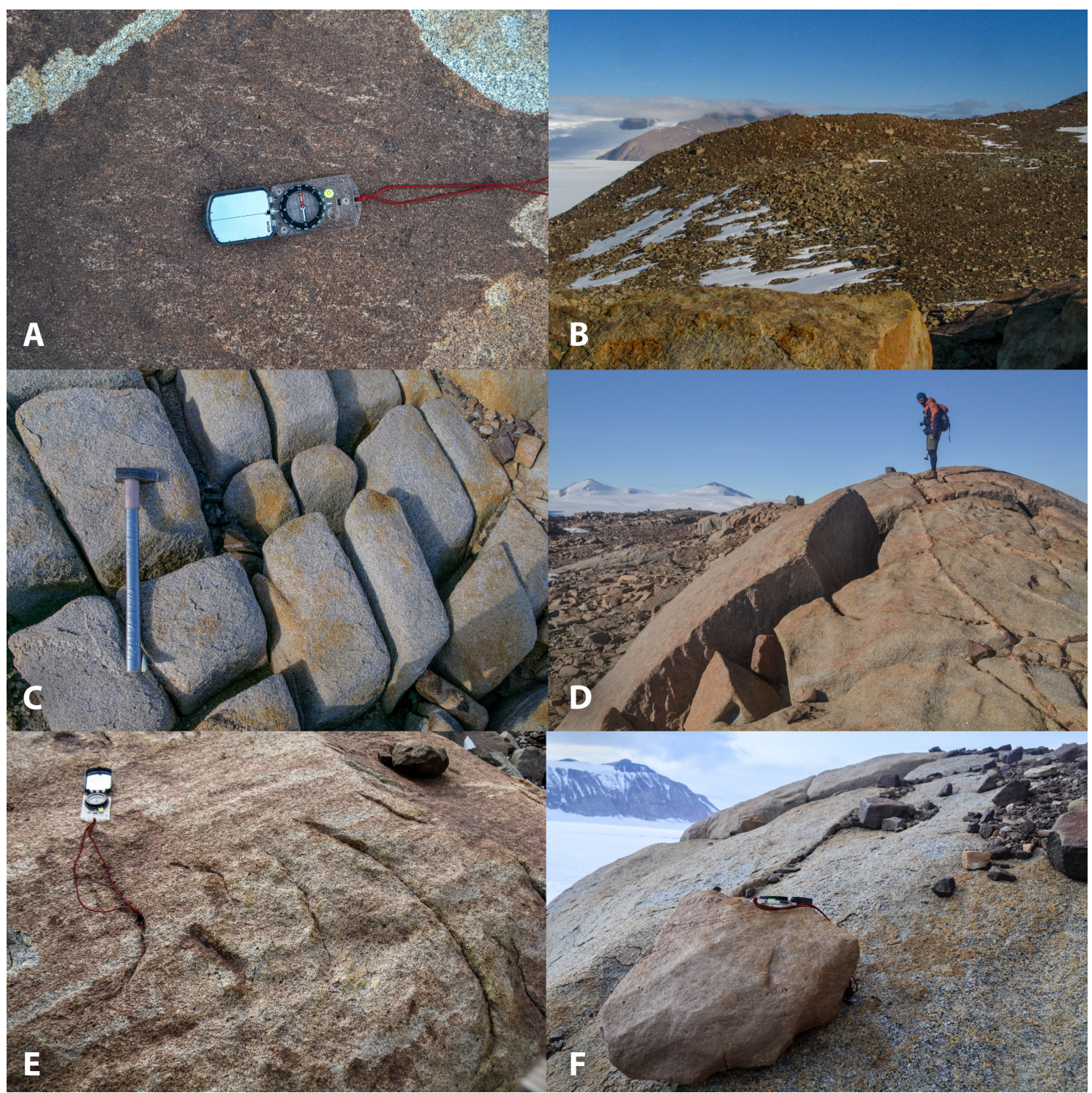

Figure 6.6 Selected representative views of geomorphic features at Mt Suess and Gondola Ridge. A) 'Fresh' striae on weathered bedrock at the higher elevation sides of Mt Suess (compass, uncorrected for magnetic north, for scale). B) Moraine ridge on Mt Suess aside cryoturbated drift deposits, looking south-east. C) Deeply weathered granite bedrock at the highest elevations of Gondola Ridge (80-cm-long hammer for scale). D) Heavily weathered and displaced, rounded bedrock at Gondola Ridge, located near C (person for scale next to perched erratic cobble). E) Relict chatter-marks and striae on granite bedrock at Gondola Ridge, with modest 'fresh' abrasions and recent spalling (compass for scale). F) Glacially-eroded erratic cobble, resting on moderately weathered rounded bedrock at Gondola Ridge (compass on cobble for scale). 


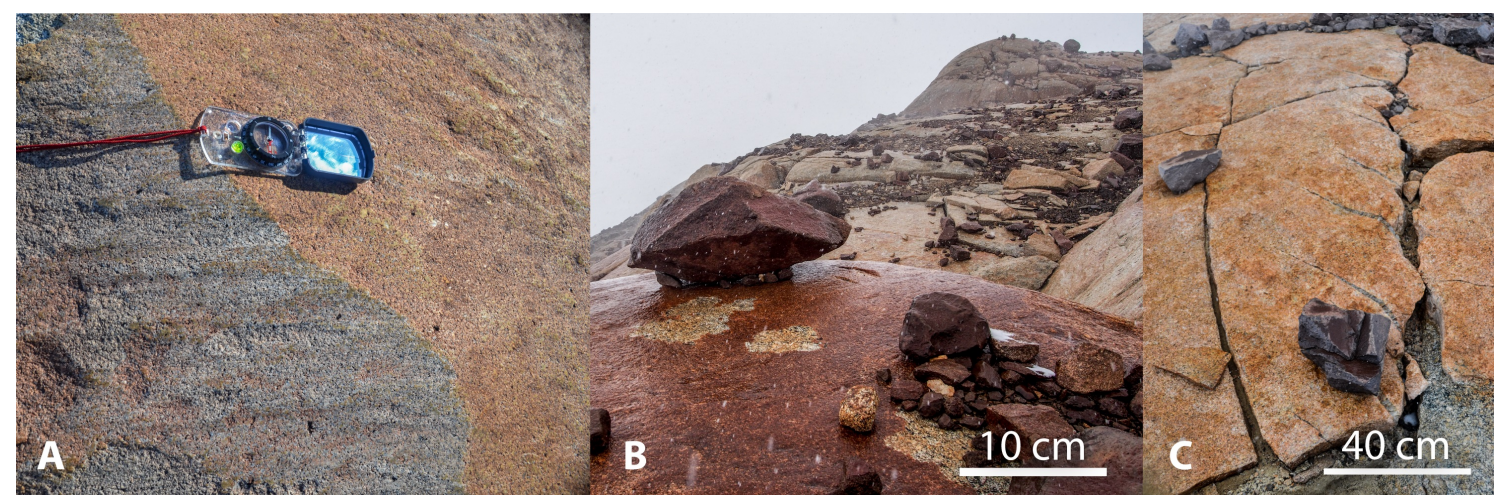

Figure 6.7 Vertical transect of the erosional and depositional signature at Mt Suess. A) Fresh erosion of a previously oxidised bedrock surface near the present-day glacier margin (compass for scale). B) Perched cobbles on rounded bedrock topography that retains 'old' striae and a heavily oxidised surface, located $\sim 100 \mathrm{~m}$ above the glacier margin. C) Heavily spalled, oxidised and fractured bedrock surfaces with a mixture of sub-rounded to angular cobbles resting on top, located $>250 \mathrm{~m}$ above the glacier margin.

grained nature of the granite, with spallation having occurred rapidly since last covered by ice. Alternatively, the bedrock surfaces may have been repeatedly weathered over multiple cycles with limited glacial erosion. The latter is supported by glacially deposited, erratic boulders perched atop heavily weathered tor-like features that are preserved on higher elevation bedrock (Figure 6.6C and 6.6D).

In summary, repeated glacial activity is evident at Mt Suess and Gondola Ridge. The vertical extent of previously thicker ice is recorded by glacial erratics and upper limits of rounded bedrock. The highest elevations of erratic cobbles indicate that the ice surface was $330 \mathrm{~m}$ above present-day in the past. Below this, 'fresh' bedrock striae and a higher concentration of erratics likely record the most recent episode of glacial cover, which was up to $260 \mathrm{~m}$ above the present-day surface. At this time, ice flowed over Gondola Ridge and around the downstream side of Mt Suess, possibly depositing a moraine and drift on Mt Suess. Glacial erosion is more evident near the modern glacier, with progressively relict, weathered surfaces preserved at higher elevations that imply cover by non-erosive ice and/or cover for a short period of time.

\subsubsection{Low Ridge \& Cuff Cape}

In the lower reaches of Mackay Glacier are two small nunataks, Low Ridge and Cuff Cape, located between the present-day grounding-line and floating terminus (Figure 6.8). Low Ridge is an elongated area of exposed granite bedrock ( 5-270 m asl), which presently divides Mackay Glacier from the less dynamic New Glacier. The top surface is weathered to a minor degree and is covered in places by till. The topography drops 
sharply on the Mackay Glacier side down to freshly eroded bedrock at the margin (Figure 6.9). A subtle lateral, and part-supraglacial, moraine of coarse, sub-rounded boulders lies parallel to the glacier margin. Erratic sandstone cobbles can be found perched on bedrock across Low Ridge (Figure 6.9A and 6.9B), and likely record the last episode of overriding ice in this location.

Cuff Cape is a small 'island' ( $<40 \mathrm{~m}$ asl) of granite bedrock that is bounded by the Ross Sea and the tongues of Mackay and New Glaciers. It is predominantly covered with drift deposits except for some exposed roche moutonnées and striated surfaces. A sequence of moraines exist on both sides of Cuff Cape (Figure 6.9C), formed from thrusting of New Glacier and from recent fluctuations of the Mackay Glacier terminus. The most recent moraine was deposited after 1913 AD (Möller, 1995) and might correlate to the moraine at Low Ridge. Both of these lower-elevation nunataks indicate complete cover during the last glacial episode with the deposition of erratics, likely sourced from upstream, and modest bedrock erosion.

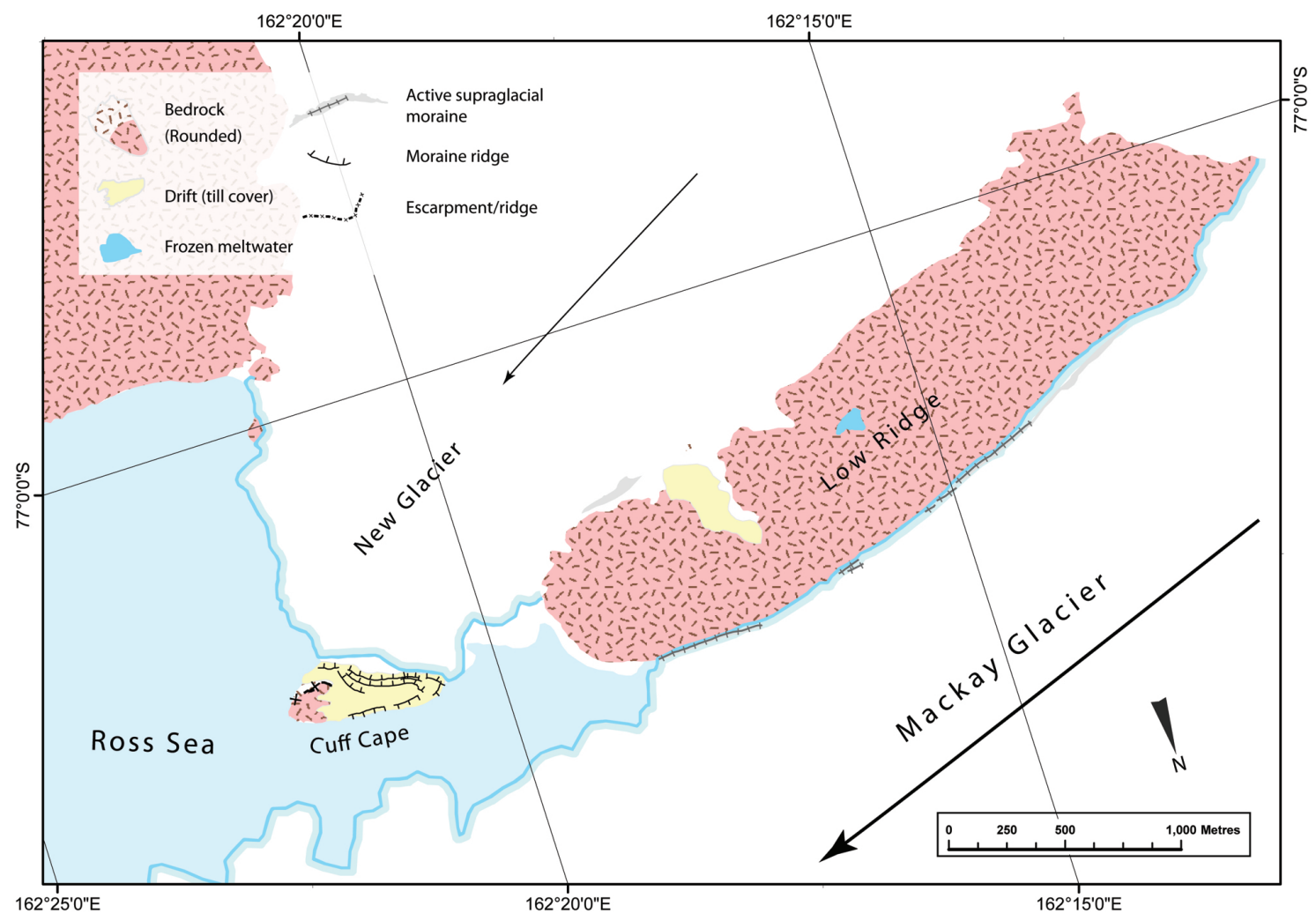

Figure 6.8 Surfical geology and geomorphology at Low Ridge and Cuff Cape, lower Mackay Glacier. Both nunataks have glacially rounded bedrock surfaces, but Cuff Cape is largely covered by recent till deposits. 


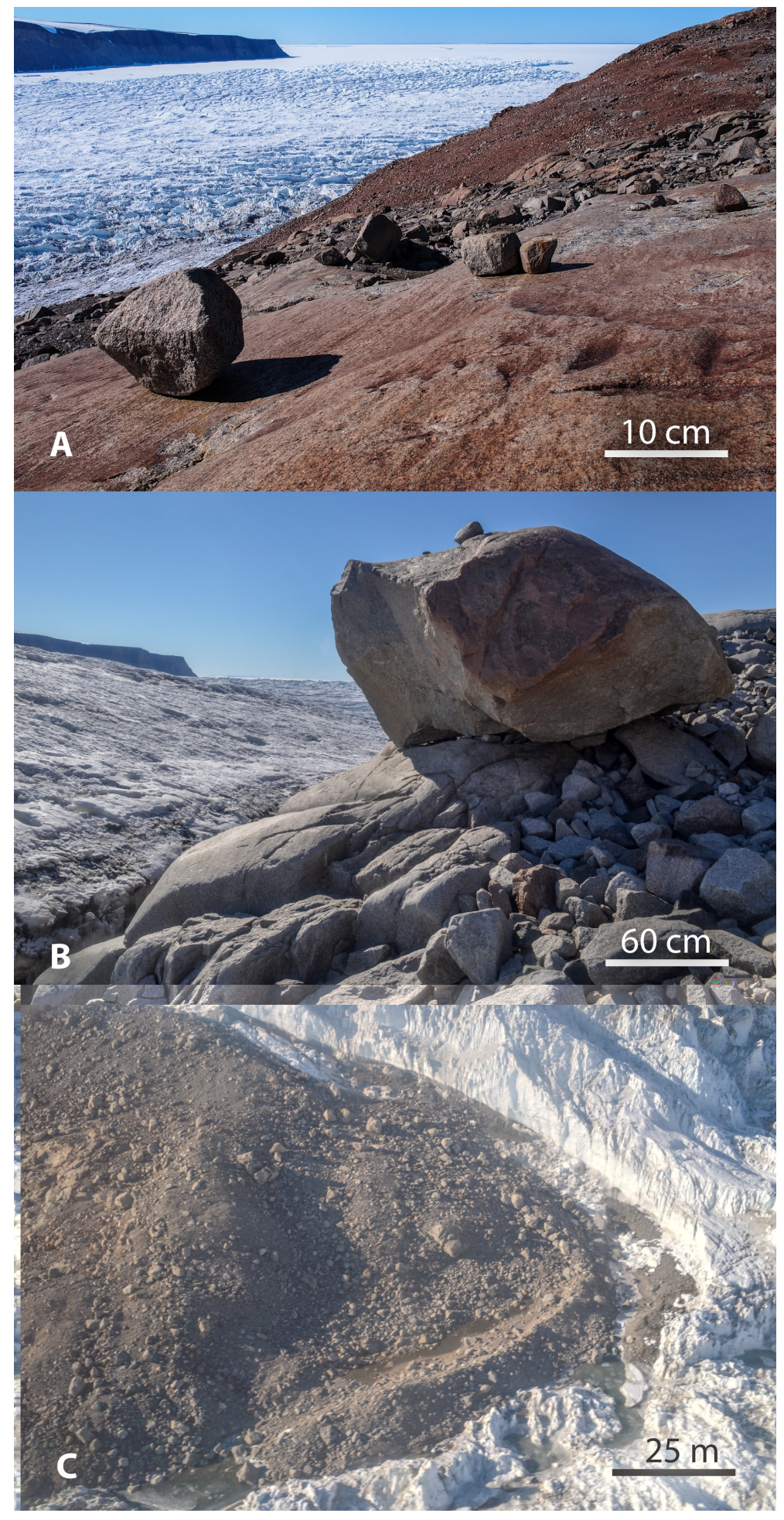

Figure 6.9 Geomorphic features at Low Ridge and Cuff Cape. A) Erratic cobbles resting on a bedrock surface with moderate recent erosion at Low Ridge ( $\sim 200 \mathrm{~m}$ above the modern glacier surface), looking north-east towards Kar Plateau. B) Erratic cobble resting on a large boulder on top of freshly eroded bedrock, at Low Ridge by the glacier margin. C) Aerial view of moraine ridges and drift deposits at the western end of Cuff Cape, with New Glacier to the top-right and Mackay Glacier to the bottom of the image. 


\subsubsection{Kar Plateau}

Kar Plateau is located on the north side of the present-day glacier terminus. The area has been mapped broadly as an elevated flat erosional surface, bounded by glacially eroded cliffs that extend up from the Ross Sea (Sugden and Denton, 2004). The plateau is divided by a distinct escarpment running south-east to north-west (Figure 6.10). Highly weathered slabs of dolerite bedrock lie above the escarpment and are thinly covered by weathered dolerite material; here, this surface has been termed 'regolith' (Figure 6.11A). Cryoturbation is a dominant process across the escarpment, but particularly on the north side at a lower elevation (Figure 6.11B). It occurs mainly from frost-heave of bedrock slabs and the turning over of overlying material, which produces extensive, well-developed polygons. Solifluction ridges and lobes have developed towards the base and on the sides of the escarpment, controlled by the slope morphology.

At the coast, ridges of clast-supported boulders run parallel to, and 10-15 m above, the sea ice edge and the base of an escarpment slope (Figure 6.11C). The boulders are large ( $>1.5$ m) and predominantly very rounded on the downslope side and more angular upslope.

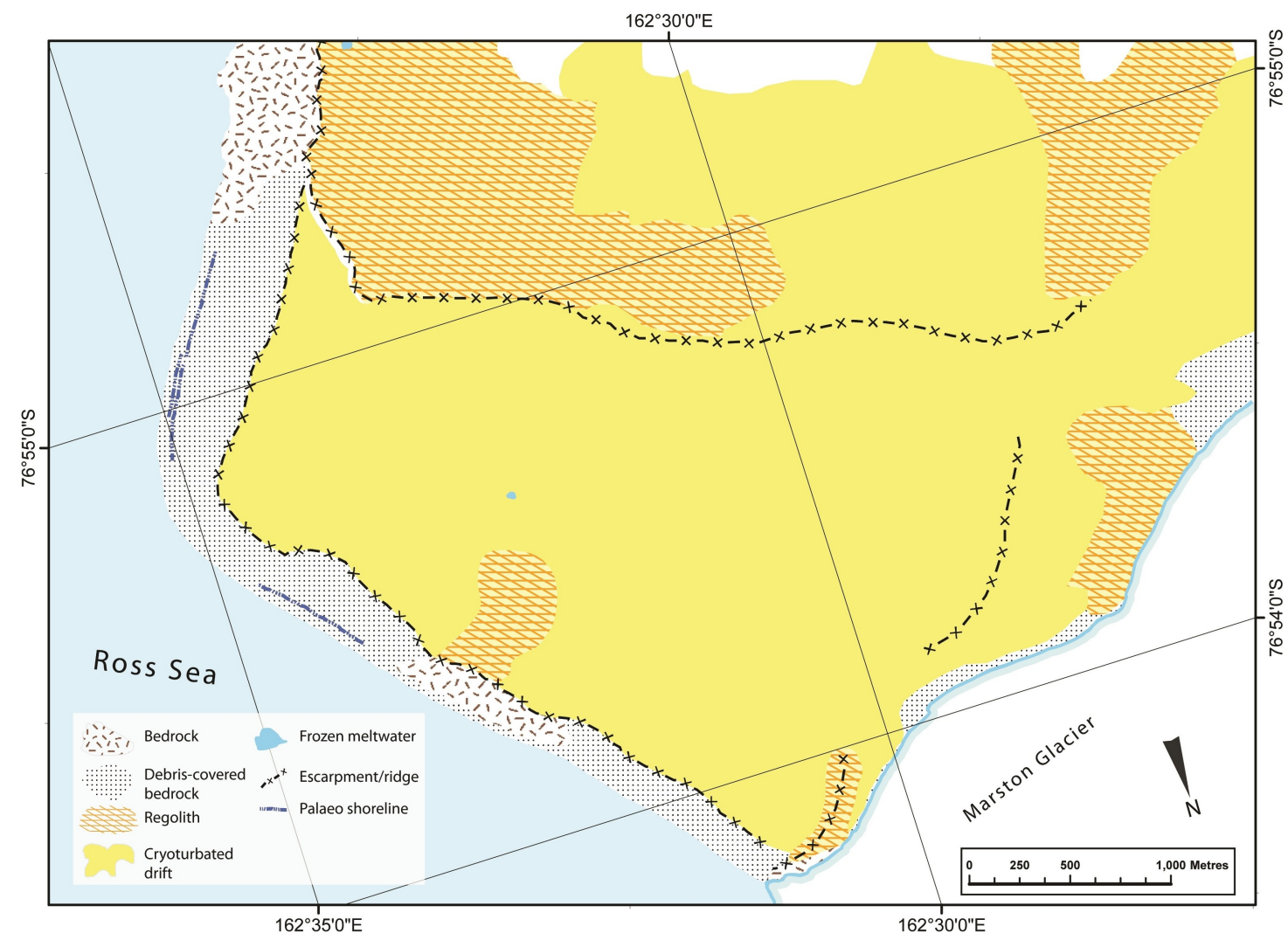

Figure 6.10 Surfical geology and geomorphology at Kar Plateau, beyond the present-day terminus of Mackay Glacier. The plateau is divided by an escarpment into an upper and lower section. Aerial weathering and cryoturbation features are extensive, with wave erosion and deposition at the margins. There are no glacial deposits preserved in situ on the plateau. 


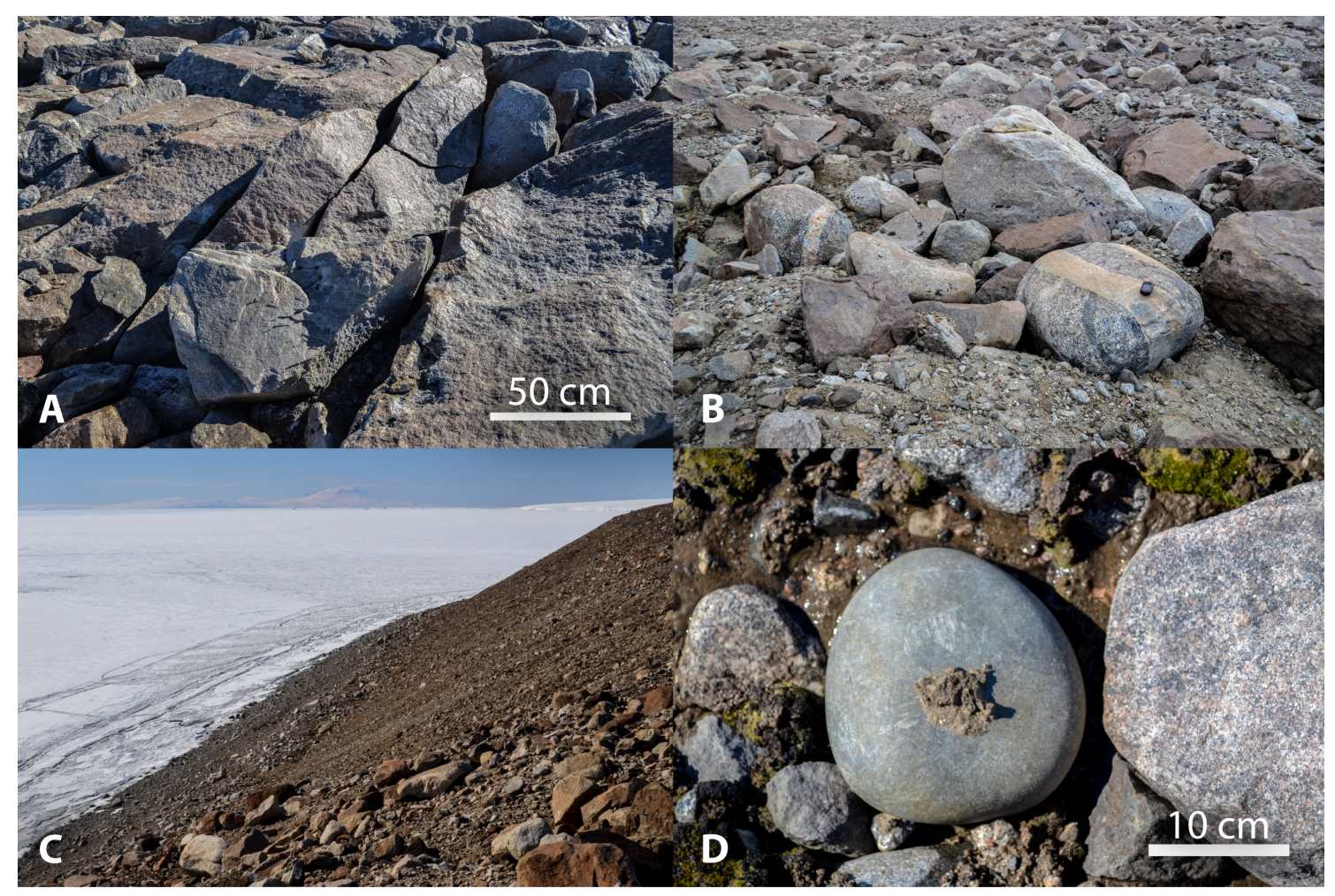

Figure 6.11 Selected representative views of geomorphic features at Kar Plateau. A) Highly fractured in situ bedrock slabs on the upper plateau, often found with weathered debris on the surface. B) Well-developed cryoturbation on the lower plateau, with angular to well-rounded boulders and elevated fine material (handheld GPS for scale).

C) Palaeo-shoreline ridge located parallel to sea margin, towards base of debris-covered escarpment (looking south-east over Ross Sea to Mt Erebus). D) Example of a well-rounded cobble with some surface abrasions.

These characteristics do not support an interpretation of storm beaches, which typically occur at $\sim 4 \mathrm{~m}$ above sea level and consist of clasts $<1 \mathrm{~m}$ in diameter (Hall et al., 2004). I instead interpret their deposition as a 'sea ice rampart' at a time of higher relative sea level, with wave action processes from below and rockfall from above. Although raised shorelines and marine limits are expected to reach about $21 \mathrm{~m}$ here (Hall et al., 2004), no wave-eroded cliffs or storm beach deposits were observed.

Past glacial extent at Kar Plateau is unclear. While there is a visual shift from brown to grey drift below the escarpment, this might represent a change of the underlying bedrock (dolerite to granite) opposed to a glacial drift limit. Glacially-transported sandstone and granite cobbles can be found across the plateau. Their surfaces have varied degrees of weathering and glacial erosion, which reflects the recycling and preservation of some material by cryoturbation processes. Numerous well-rounded cobbles are preserved on Kar Plateau up to at least $350 \mathrm{~m}$ asl (Figure 6.11D). The cobbles are characteristic of wave-eroded material, but their elevation and the presence of surface striae suggest glacial transport. 
In summary, Kar Plateau represents a surface of long-term alternating glacial erosion and subaerial weathering and modification. Determining a relative age of glacial activity is hindered by cryoturbation features, however the presence of glacial and marine derived erratics over the upper plateau indicates extensive ice cover in the past. Evidence of extensive cryoturbation on the lower plateau area may reflect more frequent or longer durations of ice cover and erosion, or a lower resistance of the granite bedrock. Palaeo-shorelines record local isostatic uplift after the last episode of glacial depression.

\subsection{Discussion}

Through assessment of the glacial geomorphology at Mackay Glacier, a window into the past ice dynamics and configurations can be inferred. In particular, there are four key interpretations; 1) an early, erosive ice sheet expansion occurred, 2) the lower reaches of the glacier were thicker-than-present during the last glacial period, with 3) both an erosive and non-erosive glacial regime, and 4) the ice flow direction has varied in the past. Additionally, cryoturbated drift is recognised as a common feature at the flanks of Mackay Glacier.

\subsubsection{Early wet-based glaciation}

Warm-based ice produces basal meltwater that in turn decreases the effective pressure and increases sliding velocity, and therefore the potential for erosion at the bed (Hallet, 1979; Cuffey and Paterson, 2010). Rounded bedrock with abraded surfaces at Mt Gran, Gondola Ridge and Low Ridge/Cuff Cape all support the idea of long-term, repeated overriding events and erosive bed-moulding ice-flow in the past. However, only at Mt Gran is there strong evidence of extensive past warm-based glaciation that has remained relatively unmodified since formation. Preserved striae and p-forms record early erosive ice cover and the presence of significant subglacial sediment flux and/or subglacial meltwater under high pressure (Glasser and Bennett, 2004). A mixture of sediment or meltwater dominated processes could explain the geomorphic signature at Mt Gran (e.g. Boulton, 1974; Shaw, 1988), although both support a warm-based regime; the production of sediment requires high erosive potential and therefore also wet-based ice within the glacial system.

Meltwater erosional forms have been identified elsewhere in Antarctica, associated with outlet glacier retreat in continental-shelf troughs (e.g. Wellner et al., 2006) and subglacial floods in the Dry Valleys (Sugden et al., 1991; Lewis et al., 2006). The formation of 
such features pertains to certain wet-based conditions, prevalent at different times since Antarctic glacial inception. Next, I will explore the physical and environmental conditions able to facilitate widespread wet-based ice at Mackay Glacier and when this warm-based regime likely existed.

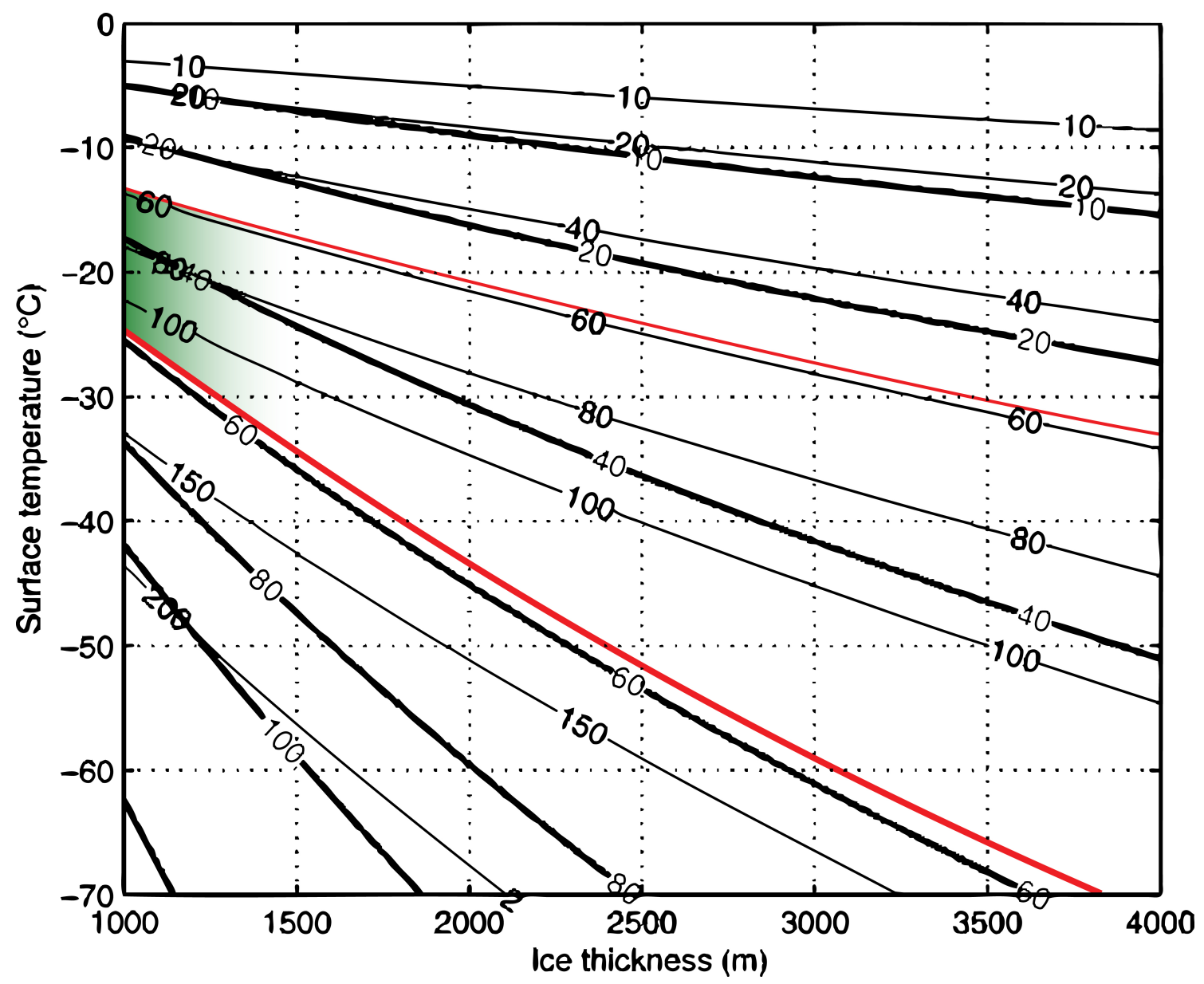

Figure 6.12 Minimum geothermal heat flux required for basal ice to reach pressure melting point as a function of ice thickness and surface temperature. The relationships are heavily dependent on whether a high vertical advection rate $\left(0.5 \mathrm{~m} \mathrm{a}^{-1}\right.$; thick line $)$ or low advection rate $\left(0.05 \mathrm{~m} \mathrm{a}^{-1}\right.$; thin line $)$ exist. Lines are annotated with corresponding heat flux values. Assuming a constant geothermal heat flux (today $\sim 58 \mathrm{~mW} \mathrm{~m}^{-2}$; red lines) and a low ice sheet thickness (today $<1500 \mathrm{~m}$ ) at Mackay Glacier, basal melt could

have occurred with annual surface temperatures between $\sim-10^{\circ} \mathrm{C}$ and $-30^{\circ} \mathrm{C}$ (highlighted in green). Modified from Pattyn (2010) with original data produced from modelling Antarctic ice sheet basal conditions.

Meltwater production requires basal ice to reach its melting point, which could occur from sufficiently thick ice or bed undulations that cause pressure melting, and/or from warm surface temperatures or high geothermal heat flux. The occurrence of basal melt is therefore dependent on the relationships between these controls (Figure 6.12), each of which will now be considered in turn. To begin, fluctuations in the geothermal heatflux are modelled to have little impact on ice sheet extent but could potentially influence basal 
temperatures and flow regime in some areas (Pollard et al., 2005). Unfortunately, this cannot be explored further without proxy data of heat flux variation through the Cenozoic.

Pressure-melting from local topography effects may in part explain the occurrence of $p$ forms on the stoss side of a bedrock high, however, cannot fully account for widespread bedrock moulding at Mt Gran and downstream sites. Poor constraints on surface elevations during the wet-based regime hamper inferences of the role of pressure melting from thicker ice. Furthermore, estimating past ice thickness is complicated by a potentially lower gradient and less incised bed profile, prior to subsequent uplift (Levy et al., 2012) and potential selective erosion of the trunk (Jamieson et al., 2010). Mackay Glacier was thicker than present during the last glacial episode, however, geomorphological evidence suggests that minimal erosion occurred at this time. Therefore, the production of meltwater at the bed suggests that these features formed during a different climate.

Elevated surface temperatures can propagate through the ice column to increase the potential for basal melt, depending on the vertical advection rate (Cuffey and Paterson, 2010). The basal temperature and basal melt rate of ice sheets are most likely to peak towards the end of a glacial-interglacial cycle, when surface temperature increases but a large ice volume still exists (e.g. Marshall and Clark, 2002). It can be tentatively deduced that a combination of relatively warm surface temperatures and thicker ice during previous glaciations facilitated wet-based, erosive ice. Annual surface temperatures as warm as $-10^{\circ} \mathrm{C}$ could have produced wet-based ice, with thicker ice able to reduce the surface temperature required (Figure 6.12).

Warmer than present conditions and extensive ice cover most likely occurred in the region of Mackay Glacier during the Miocene. Based on marine records of benthic $\delta^{18} \mathrm{O}$, the modelled maximum Antarctic ice volume occurred at $\sim 18 \mathrm{Ma}$ (Pekar and DeConto, 2006). Interestingly, geological evidence in the Ross Sea sector suggests that the maximum expansion of ice occurred during both the Early and Middle Miocene. An offshore sediment core collected downstream of Mackay Glacier records a substantial erosional hiatus at 23.7 Ma (Naish et al., 2001), which is supported by evidence of grounded ice that reached the outer continental shelf at about that time (Bartek et al., 1997). However, in the Dry Valleys and Convoy Range, the largest overriding event is recorded from tephra draped on glacial deposits at 14.8-13.6 Ma and 13.62-12.44 Ma (Sugden and Denton, 2004; Lewis et al., 2007). It is therefore apparent that significant erosive and depositional glaciations occurred during the Miocene in the vicinity of Mackay Glacier. However, the spatially variable timings and geologic representation of such glaciations prevent the affiliation of Mackay Glacier's erosive geomorphic signature to a single Miocene glacial episode. 
Miocene and Pliocene deposits in the region of Mackay Glacier also indicate the past basal conditions of East Antarctic glaciers through time. Local glaciers in the Transantarctic Mountains show a change in till composition at 13.94 Ma that records a switch from warm-based to cold-based ice (Lewis et al., 2007). Evidence for the existence of warmbased ice prior to the Mid-Miocene transition is also present in the Ross Embayment, where offshore sediment records reveal that EAIS outlet glaciers were erosive and produced meltwater before a change to cold polar regimes (McKay et al., 2009; Passchier et al., 2011). However, another period of potentially warmer basal conditions is then recorded during the Late Miocene with the characteristics of a subpolar regime, before a final transition back to a polar regime during the Early to Late Pliocene (McKay et al., 2009). Despite widespread cold-based ice expansion during both the Miocene and Pliocene, warm-based glacier trunks may have facilitated selective erosion within outlet glaciers at this time (Jamieson et al., 2010). The Mt Gran site lies at the flank of Mackay Glacier and is positioned at the base of a high massif that indicates it probably never existed as the bed of the central glacier trunk. Therefore, the wet-based conditions interpreted at Mt Gran probably occurred during either the Early or Late Miocene, associated with high meltwater and basal erosion, and increased sediment supply to the Ross Sea.

In summary, geomorphic evidence suggests a previous warm-based, erosive glacial regime during a period of warmer atmospheric conditions. At that time, meltwater production, erosion potential and sediment flux likely peaked towards the end of glacial-interglacial cycles as surface temperatures increased. Geological evidence in the vicinity of Mackay Glacier suggests that this wet-based regime likely occurred during the Early and/or Late Miocene, but may have extended into the Pliocene with regional variability (Smellie et al., 2011).

\subsubsection{Extent and erosion during the last glaciation}

The last significant glacial episode is recorded in the vicinity of Mackay Glacier at the LGM (Anderson et al., 2014). Radiocarbon ages obtained from glacial deposits at the sides of McMurdo Sound indicate that grounded ice possibly reached its maximum thickness in this area at $\sim 26.8{ }^{14} \mathrm{C} \mathrm{ka}$, and remained in that configuration until $\sim 23.8-12.7$ ${ }^{14} \mathrm{C}$ ka (e.g. Denton and Marchant, 2000; Hall and Denton, 2000). Immediately south of Mackay Glacier, a well-dated relative sea level curve records grounded ice that remained until $\sim 6.8{ }^{14} \mathrm{C}$ ka along the Scott Coast (Hall et al., 2004). Furthermore, offshore studies suggest the Mackay Glacier tongue was more extensively grounded during that time and in the relatively recent past, reflected in a diminished epibenthic community and the occurrence of flutes and grounding-zone wedges (Powell et al., 1996; Dawber and Powell, 
1997; Greenwood et al., 2012).

At Mackay Glacier, thicker ice is inferred at a suite of terrestrial sites from glacial deposits and bedrock erosion. Based on the highest elevations of 'fresh' striae, perched erratics and a moraine within glacial drift, the LGM surface elevation was approximately $>1045 \mathrm{~m}$ asl (>115 $\mathrm{m}$ higher than the modern ice surface) at Mt Gran, $825 \mathrm{~m}$ asl (260 $\mathrm{m}$ higher) at upstream Mt Suess, $\sim 765 \mathrm{~m}$ asl (220 $\mathrm{m}$ higher) at downstream Mt Suess, and $>270 \mathrm{~m}$ asl ( $>260 \mathrm{~m}$ higher) at Low Ridge. This revises the previous LGM surface elevation of $150 \mathrm{~m}$ above present, estimated from glacial drift at Mt Suess (Denton and Hughes, 2000). A refugia of small arthropods (springtails) has existed on Mt Suess since initial isolation 3-5 Ma ago (I. Hogg, pers. comm.), which supports evidence that ice did not completely cover Mt Suess at the LGM, nor possibly during any previous glacial episode. A lack of high-elevation topography with a potential LGM signature prevents delimitation of the surface in the lower reaches of the glacier. A geologically-constrained surface profile of grounded ice in the Ross Sea suggests that the LGM ice surface existed somewhere between 400 and $700 \mathrm{~m}$ asl at the mouth of Mackay Glacier (Denton and Marchant, 2000; Orombelli et al., 1990; Denton and Hughes, 2000). Continental-scale ice sheet models simulate a surface $>100 \mathrm{~m}$ higher than these reconstructions (e.g. Huybrechts, 2002; Golledge et al., 2012; Whitehouse et al., 2012; Briggs and Tarasov, 2013), with a recent modelling study providing an improved surface elevation estimate in the vicinity of Mackay Glacier of 600-700 m asl (e.g. Golledge et al., 2013). By plotting the locations and elevations of new geomorphological evidence, a surface profile can be reconstructed down Mackay Glacier (Figure 6.13). This surface is consistent with estimates beyond the mouth of the glacier (at $\sim 400-700 \mathrm{~m}$ asl) and supports the idea that Mackay Glacier was buttressed by grounded ice in the Ross Sea at the LGM.

The LGM configuration of the glacier would have accommodated different basal conditions to those of today. Driving stress, and therefore the basal shear stress and sliding velocity, increases with thicker and steeper ice. The reconstructed surface profile of Mackay Glacier shows thickening and a reduced surface gradient in its lower reaches at the LGM. Therefore, if thicker ice is the dominant factor then basal shear, sliding and erosion should have been greater further downstream, or vice versa if the low surface gradient controlled friction at the bed. We can observe this relationship downstream in the contrasting geomorphic signatures between Mt Gran and Low Ridge/Cuff Cape. At Mt Gran only surficial, shallow ('fresh') striae were observed, indicating minimal bedrock erosion. Such striae are characteristic of slow abrasion beneath cold-based ice (Shreve, 1984; Augustinus et al., 1997; Atkins et al., 2002). Sliding is minimal but still possible under these conditions, requiring a thin film of water and fine sediment at the ice-rock contact, and has been observed in glaciers with sub-freezing basal temperatures (e.g. Echelmeyer and Zhongxiang, 1987; Cuffey et al., 1999). 


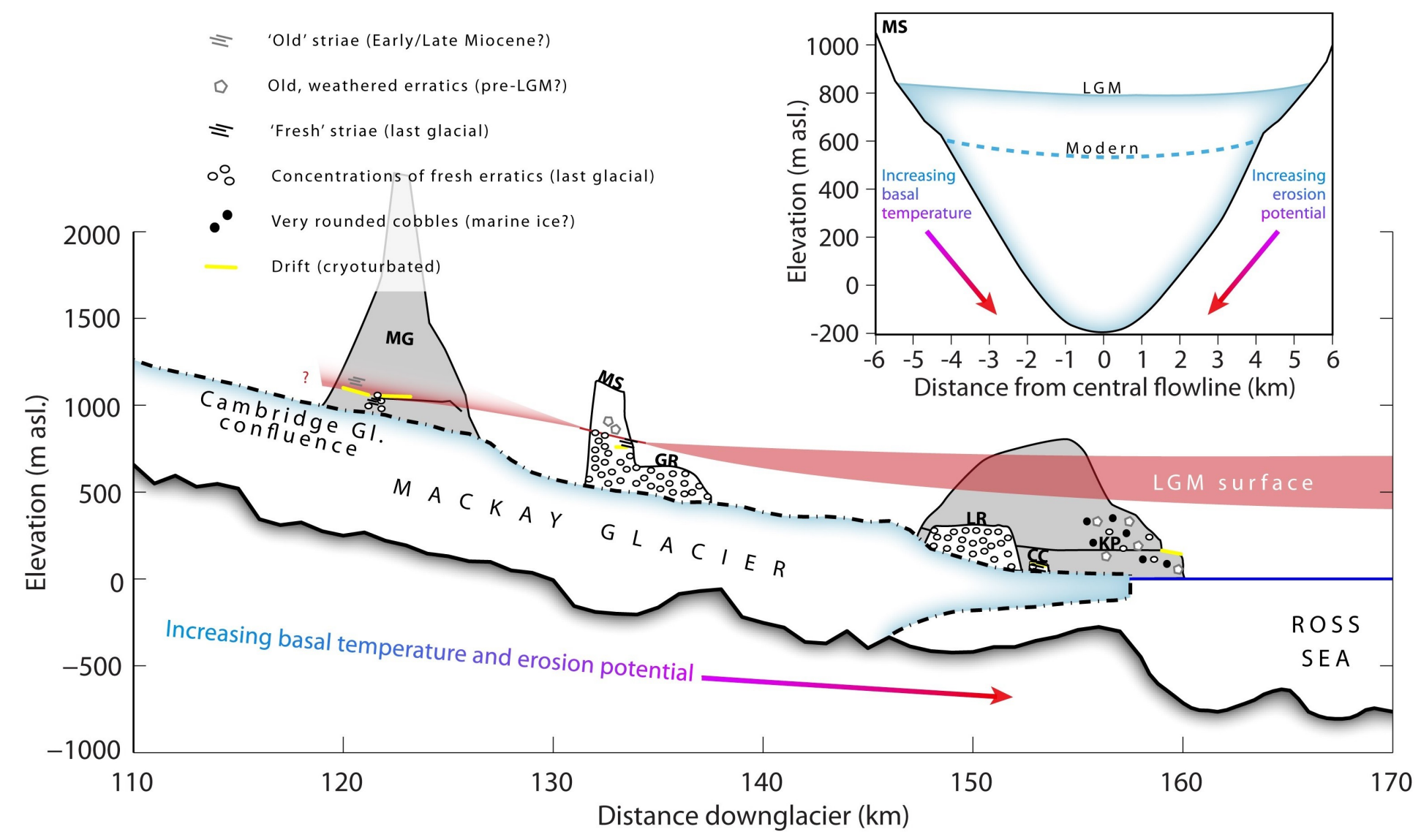

Figure 6.13 Overview of glacial geomorphology down-glacier and a reconstructed profile of Mackay Glacier at the LGM. Evidence is presented schematically at Mt Gran (MG), Mt Suess (MS), Gondola Ridge (GR), Low Ridge (LR), Cuff Cape (CC) and Kar Plateau (KP). An estimated LGM surface has an unknown upper bound at Mt Gran and is constrained by previous geological estimates (400-700 m asl) beyond the present-day terminus. The geomorphic signatures indicate increasing basal temperature and erosion potential towards the terminus and the centre of the Mackay Glacier trunk at the LGM. The modern surface profile is derived from airborne radar (ICECAP, University of Texas Institute for Geophysics), while the bed profile is obtained from BEDMAP2 (Fretwell et al., 2013) and corrected with local ice depth estimates derived from seismic data (Calkin, 1974). The inset shows a vertical transect profile of Mackay Glacier at Mt Suess. 
Downstream at both Low Ridge and Cuff Cape, the last overriding event formed roche moutonnées and removed any 'old' striae from an earlier glaciation. This evidence supports higher basal shear stress and bedrock erosion at Low Ridge and Cuff Cape, as a result of the increased weight of overriding ice. However, surface erosion of heavily oxidised, relict surfaces was incomplete in some places, indicating only minor meltwater production to facilitate modest bedrock erosion. It is therefore likely that Mackay Glacier was predominantly cold-based along its length, with an increased potential for warm-based ice and water at the bed further downstream due to thickening of the glacier.

Despite evidence that Mackay Glacier has in recent times been an inefficient agent of erosion, glaciated erratic cobbles with negligible surface weathering can be found at all sites alongside Mackay Glacier. Such deposits would require either upstream subglacial plucking, or surface erosion of a previously deposited boulder during the LGM. It is unlikely that these erratic clasts underwent fresh glacial erosion without being transported out of the catchment; assuming a low average velocity of $5 \mathrm{~m} \mathrm{a}^{-1}$, a freshly eroded cobble from a pre-LGM glacial regime would have been removed from the $\sim 160-\mathrm{km}$-long system in $<32 \mathrm{ka}$, which is within the length of a glacial-interglacial cycle. This suggests that subglacial plucking occurred and, therefore, parts of Mackay Glacier were likely warm-based at the LGM.

Mt Suess and Gondola Ridge reveal a vertical geomorphic gradient between the modern surface and the high-elevation rectilinear slopes during the last glacial episode. Fresh erosion of rounded bedrock is observed near the ice margin, which grades into rounded bedrock with heavily oxidised surfaces and 'old' abrasions, and then into rounded but heavily weathered and fractured bedrock surfaces at high elevations. This vertical gradient could be explained by 1) time-dependent weathering, 2) elevation-dependent environmental factors, or 3 ) erosion dependent on the glacier's thermal regime.

During thickening and then thinning of Mackay Glacier, the upper flanks would have been exposed for a longer time than the present ice marginal surfaces, and therefore would be subjected to a longer period of weathering. While this could explain the contrast in surface oxidisation between elevations, relict abrasions are imprinted in the surfaces suggesting preservation rather than erosion during glacial cover. Environmental controls may additionally influence the degree of surface weathering, although these effects were most likely minimal over the observed elevation range. Surface spallation and rock fracturing are common in Antarctic upland areas, and typically occur from temperature-induced expansion and contraction (e.g. Hall, 1997b; Hall and André, 2001; Hall et al., 2002). The higher elevations at Mt Suess may have experienced less shade and more insolation, and therefore a greater range of surface temperature, and potential for rock volume change. The apparent vertical gradient could therefore, in part, be the result of weathering 
variations with both elevation and time exposed. However, the graded erosion signature observed at the flanks of Mackay Glacier indicates greater erosion at lower elevations with greater proximity to the centre of the glacier trunk.

Together with the presence of glaciated erratic cobbles, evidence of an erosional gradient suggests that the LGM glacier contained warm-based ice in the central trunk and thin, cold-based ice on the upper flanks (Figure 6.13). This is consistent with that modelled for large Transantarctic Mountain outlet glaciers at the LGM (Golledge et al., 2013). A polythermal glacier regime with a spatially-variable pattern of basal erosion is similarly reconstructed near the margins of past Northern Hemisphere ice sheets; surface-exposure dating revealed warm-based, erosive ice both in the central trunk (Briner et al., 2006) and towards the terminus (Dühnforth et al., 2010). Here, geomorphological assessment highlights that relatively small Antarctic outlet glaciers can exhibit polythermal regimes during glacial maximum configurations. Specifically, the increased thickness of Mackay Glacier facilitated areas of warm-based ice that enabled wet-based erosion of the bed in the central mid-lower reaches, despite a shallow surface profile and colder surface air temperatures during the last glacial episode.

\subsubsection{Evidence of past flow directions}

As today, the flow direction of Mackay Glacier would have been controlled laterally by bedrock relief in the past. It would also have been dependent on the evolving ice thickness and basal conditions. Under widespread wet-based conditions, basal drag is reduced and the glacier can flow more freely downstream over the bed topography. This might explain the more direct downstream flow recorded at Mt Gran, as differing orientations between 'old' and 'fresh' striae, during early wet-based glaciation. Flow is also controlled by the ice surface slope and, therefore, the different striae orientations may reflect a past change in the prevailing ice sheet surface slope. Alternatively, Pliocene-to-present uplift (Levy et al., 2012) and repeated erosion beneath the central glacier trunk over multiple glacial cycles may have encouraged ice to flow more towards the central trunk at the LGM, reflected in the orientations of 'fresh' striae.

The modern Mackay Glacier flows sinuously downstream, laterally confined by highrelief mountain ranges and central nunataks. During the LGM, the increased ice thickness enabled ice to flow over Gondola Ridge towards the Ross Sea, but also around Mt Suess to the south-east. As ice-flow direction is typically controlled by surface slope, thinner ice probably occupied the south-east side of Mt Suess and Gondola Ridge with a much lower surface relative to the main trunk of Mackay Glacier. This is contrary to the LGM glacier 
geometry of a recent ice sheet modelling study (Figure 6.2; Golledge et al., 2013), where instead thick ice is simulated in this area parallel to the trunk, supplied from upstream and buttressed downstream by grounded ice in the Ross Sea. However, part of this discrepancy is probably derived from the 5-km grid resolution, imperfect climate inputs and smoothing of the bed for ice-sheet simulations. Today, ice in this area is sourced from Cotton Glacier and other smaller glaciers in the Clare Range and Gonville and Caius Range on the northern edge of the Dry Valleys. It is therefore likely that these local glaciers were similar in size to today during the LGM, if not smaller. The climate of the Dry Valleys had sufficiently low precipitation and/or enhanced ablation conditions at the LGM to limit the expansion of local glaciers (Denton and Hughes, 2000; Denton and Marchant, 2000; Hall et al., 2000), including those areas to the north that skirt Mackay Glacier.

During glacial episodes, ice became grounded in the Ross Sea (e.g. Anderson et al., 2014; Naish et al., 2009) and buttressed the flow of outlet glaciers into the Ross Embayment (e.g. Bockheim et al., 1989). However, the flow conditions at the confluences of these ice masses is not well known. Well-rounded boulders and pebbles observed at Kar Plateau suggest transport from a marine location inland to higher elevations, possibly by ice. Isostatic uplift alone cannot explain the occurrence of these clasts beyond $350 \mathrm{~m}$ asl. In McMurdo Sound at the LGM, grounded ice in the Ross Sea encroached into the mouth of Taylor Valley (Denton and Hughes, 2000; Denton and Marchant, 2000), at a time when a starved Taylor Glacier was unable to extend beyond the Transantarctic Mountains. This differs from Mackay Glacier, where offshore lineations and grounding-zone wedges record an advanced position and confluence with other grounded ice north of Ross Island at the LGM (Greenwood et al., 2012). It is therefore unlikely that ice grounded in the Ross Sea flowed towards Kar Plateau to deposit these marine-derived clasts at the LGM. Instead, such deposition probably occurred prior to the LGM, when Kar Plateau was overridden by grounded ice from the Ross Sea, or the relative sea level was much higher.

\subsubsection{Cryoturbated geomorphology}

Cryoturbated drift deposits and weathered debris are common on the flanks of Mackay Glacier, and are particularly extensive on the low gradient, aerially scoured surfaces at Mt Gran and Kar Plateau. Such periglacial terrace features are often associated with arid conditions, low dynamism of sediments and a long period of development (Hall, 1998). It is not clear whether these well-established deposits formed over multiple glacialinterglacial cycles or since the LGM. This is further discussed below.

Periglacial activity is dominantly controlled by climate, especially by moisture availabil- 

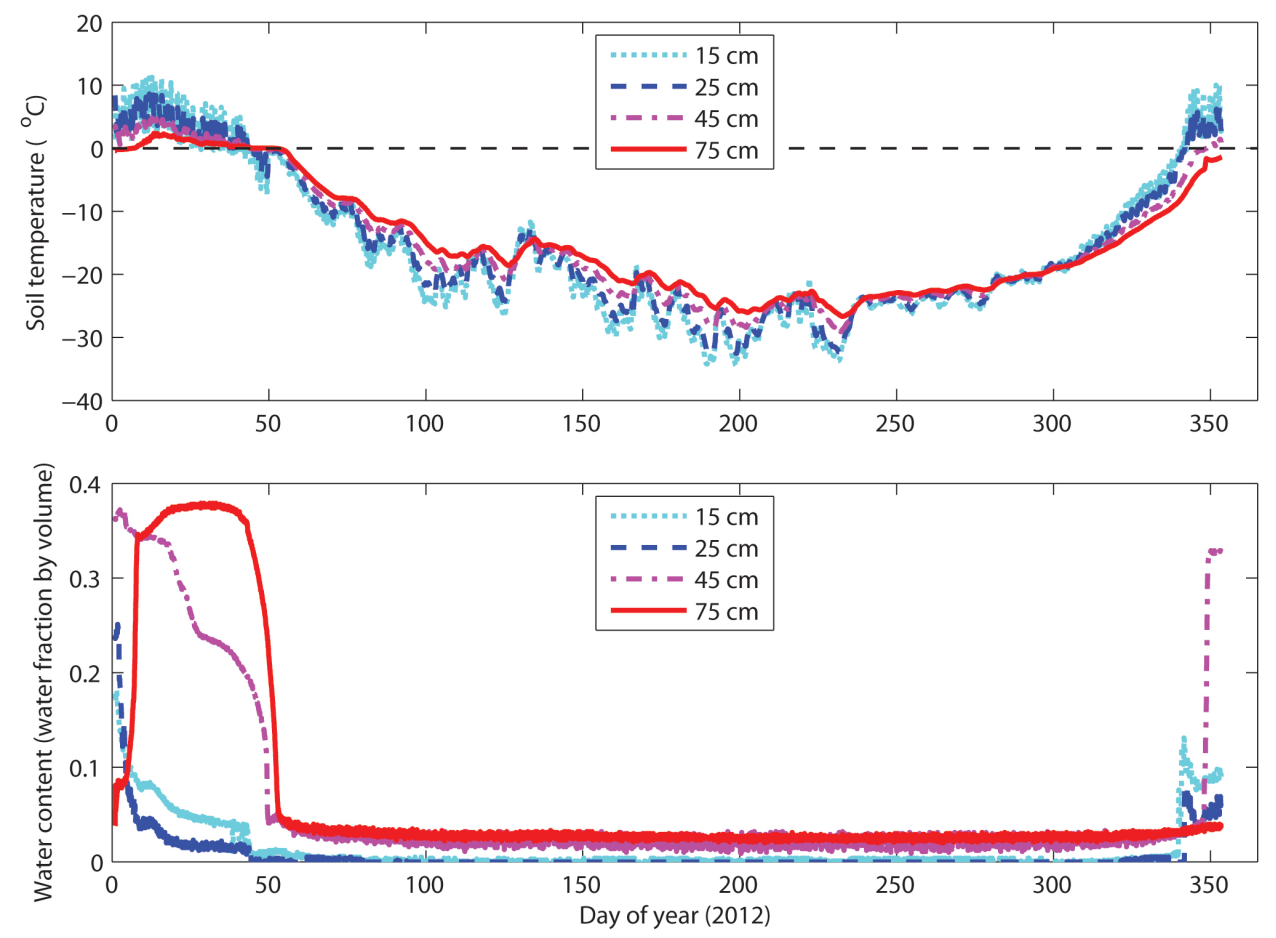

Figure 6.14 Ground climate as soil temperature and water content at Granite Harbour, mouth of Mackay Glacier. Soil temperature reaches the melting point of water during the peak 2 months of summer, with a more lagged response and diffuse effect at greater depths (upper panel). This corresponds to a summer peak in water content from melt at lower depths (45 and $75 \mathrm{~cm}$ ) (lower panel). Moisture is frozen in the ground during autumn-spring and depleted in the cold and arid near-surface soil depths (15 and $25 \mathrm{~cm}$ ). Data was obtained from National Soil Survey Center / Landcare Research as an example year (2012) of ground temperature-melt relationships.

ity and temperatures fluctuations around the freezing point of water. Cryoturbation in the region of Mackay Glacier occurs predominantly in coastal areas where there is abundant soil moisture, rather than in the more arid Dry Valleys and upland areas (Bockheim, 2002). Lower elevations in Antarctica are also more likely to experience freeze-thaw activity as ground temperatures warm to above water's freezing point. Weathering and cryoturbation are dependent on the intensity, magnitude and length of freeze-thaw cycles, which are a function of the temperature range variation and ground water content (e.g. Vliet-Lanoë et al., 1988, 1991; Hall et al., 2002), and in turn, control the degree of ground disturbance; the warmer the permafrost and greater the amount of ice in the ground, the greater the deformation (French, 2007).

Today, Granite Harbour, at the mouth of Mackay Glacier, has a mean annual temperature of $-17 \pm 2{ }^{\circ} \mathrm{C}$ with an annual temperature range of $35^{\circ} \mathrm{C}$ (2003-2012) (data obtained from 
the National Soil Survey Center/Landcare Research). Frequent freeze-thaw cycles can drive mechanical weathering, but under such low mean annual temperatures weathering rates tend to be very low (Matsuoka, 1991). Furthermore, the degree of cryoturbation depends on the response of the frozen ground to temperature fluctuations. While Granite Harbour experiences variations in soil temperature that reflect changes in atmospheric temperature, these are below $0^{\circ} \mathrm{C}$ for the coldest $\sim 10$ months of the year (autumn-spring) (Figure 6.14). Thaw occurs in the active layer to depths below $50 \mathrm{~cm}$, which has been shown to produce annual slumping of frost-heaved ground and, to a lesser extent, upward movement from frost heave when the ground is frozen (Matsuoka and Hirakawa, 2000). Freezing appears to occur from the surface down at Granite Harbour and when frozen, the near-surface soil has negligible moisture content (Figure 6.14), which reflects the extremely dry atmosphere at this coastal site with the expansion of winter sea ice. A single, seasonal peak of ground water content occurs in the summer, indicating low frequency freeze-thaw activity that is possibly of high magnitude as thaw occurs at depths of at least $75 \mathrm{~cm}$. Therefore, today, the cryoturbation of drift at Mackay Glacier most likely occurs, but not at a tremendous rate.

A range of geneses could explain the observed cryoturbated features. Periglacial surface deposits can deform from long-term creep of permafrost under gravity. Movement is typically very slow at high latitudes, with creep rates in the order of $0.03-0.05 \mathrm{~cm} \mathrm{a}^{-1}$ (French, 2007), or 3-5 m per $10 \mathrm{ka}$. Solifluction can develop uniformly without vegetation, forming either sheets or lobes depending on moisture availability. Assuming aridity increases with distance from the coast, the inland location of Mt Gran is more suited to the formation of solifluction sheets and this may explain the possible cross-terrace downslope movement of drift here. In contrast, the solifluction lobes noted at coastal Kar Plateau could be explained by more readily available moisture that can penetrate the ground to greater depths. Solifluction alone cannot explain the origin of the cryoturbated drift nor its widespread distribution, on most bedrock surfaces.

The concept of "cryoplanation" has previously been applied to terraced areas of flat and stepped surfaces in Antarctica (e.g. Hall, 1997a; Matsuoka, 1995). Thought to be initiated by nivation processes (Thorn and Hall, 2002), a slope becomes subject to intense frost action and then the downslope transport of debris by solifluction (Hall, 1998). Cycles of exposure, frost-action and solifluction continue until the landscape reaches maturity, at which point the landscape is mantled in debris (Peltier, 1950). The cryoturbated drift at both Mt Gran and Kar Plateau appear to be 'mature' cryoplanation surfaces with a mixture of locally weathered material and debris redistributed by solifluction processes. At Kar Plateau, the stepped profile likely represents the lithological boundary between dolerite and granite, with the granite of the lower plateau more substantially modified. This boundary may continue to be exploited in the future as frost-action occurs under 
the escarpment snow banks, steepening the slope (Hall, 1998; Thorn and Hall, 2002). It is therefore likely that the observed distribution of drift resulted from a mixture of frost action and solifluction processes, in association with development of the underlying substrate.

In summary, combined assessment of the observed cryoturbation features and the modern climate conditions at Mackay Glacier suggest that it is unlikely that this cryoturbation developed during a single interglacial episode, such as the Holocene. Today, mature cryoturbation terraces exist on the flanks of Mackay Glacier, yet the low moisture and annual freeze-thaw environment likely inhibits high rates of weathering, erosion and mass movement. During glacial-interglacial transitions, lower temperatures and moisture availability would have higher limiting effects on the cryoturbation potential. Under warmer atmospheric conditions than present, for example $>5^{\circ} \mathrm{C}$ (Roberts et al., 2003; Prebble et al., 2006), the ground would spend less time frozen with more frequent freeze-thaw cycles and possibly higher moisture availability, resulting in greater cryoturbation. While the age of the cryoturbation deposits cannot currently be determined, it can be inferred that their development has occurred over multiple interglacials. Together with the presence of possible pre-LGM marine-derived material, these mature cryoturbation deposits support limited subglacial erosion at Mt Gran during the LGM, but also limited erosion at Kar Plateau, away from the central trunk of the lower Mackay Glacier.

\subsection{Conclusions and wider implications}

The past configurations and dynamics of Mackay Glacier can be inferred from spatially variable geomorphic signatures. At least two contrasting glacial regimes are evident during previously expanded configurations: 1) predominantly warm-based erosive ice, which moulded most of the lower-lying ice-free areas flanking the modern glacier during a warmer-than present climate; and 2) predominantly cold-based ice with negligible erosion at the flanks and likely warmer basal ice and increased erosion towards the central trunk and terminus. The timing of the former glacial regime can only be constrained to likely Early or Late Miocene, or possibly Early Pliocene, by palaeoclimate and geological reconstructions in the vicinity of Mackay Glacier. The latter glacier regime produced a widespread signature contemporary with the most recent episode of overriding ice, which is assumed to be of the LGM and subsequent deglaciation based on regional ice sheet reconstructions (and Chapter 7). At this time, the basal erosion was focused in the central trunk of the glacier and towards the terminus, suggestive of a polythermal regime. Similar basal conditions are suggested at deglaciated Northern Hemisphere sites during the LGM 
(e.g. Briner et al., 2006; Dühnforth et al., 2010). A polythermal glacier regime is also consistent with ice-sheet model derived simulations of large Antarctic outlet glaciers (e.g. $>20 \mathrm{~km}$ wide), but not with simulations of small outlet glaciers (e.g. $<10 \mathrm{~km}$ wide) that were predicted to be uniformly cold-based (Golledge et al., 2013).

The geomorphic interpretations presented here suggest that ice thickness is the primary control on basal erosion and that small outlets with sufficiently deep trunks, such as those that intersect the Transantarctic Mountains, can support warm-based sliding and erosive ice under glacial maximum configurations. As a result, the dynamic response of smaller outlet glaciers may not be well represented by coarsely-gridded ice sheet simulations.

The inferred LGM thickness of Mackay Glacier enables assessment of the past ice sheet configuration and volume. Glacial deposits and erosion signatures as well as independent constraints from biological age markers and regionally dated geological reconstructions suggest a glacier surface between $\sim 250 \mathrm{~m}$ and $400-700 \mathrm{~m}$ above present in the lower reaches of Mackay Glacier with a diminished thickening upstream at the LGM. While this new ice surface evidence is higher than previous ice surface estimates (e.g. Denton and Hughes, 2000), reconstructed LGM surfaces in this region are still several hundred metres lower than modelled estimates (e.g. Golledge et al., 2012; Whitehouse et al., 2012), including locally-biased simulations that incorporate enhanced ablation centred over the Dry Valleys (Golledge et al., 2013). The implication is that a very low gradient ice sheet surface existed in the western Ross Sea, extending to near the continental-shelf edge, with associated low driving stresses. However, an LGM grounding-line in the Ross Embayment is only tentatively constrained (Anderson et al., 2014). Alternatively, the modestly elevated LGM surfaces that are suggested at terrestrial sites may instead indicate a less extensive LGM ice sheet and therefore steeper ice surface slopes to the grounding-line, which would require more glaciologically feasible driving stresses. Together, the geologic evidence in the western Ross Embayment supports thicker ice at the LGM, but like other sectors of Antarctica (e.g. Mackintosh et al., 2011), an insufficient ice volume to source large deglacial meltwater pulses (Carlson and Clark, 2012). While the presence of past thicker ice at Mackay Glacier can be interpreted from the geomorphology, the timing of the onset and rate of thinning following the LGM is unknown.

Temporal constraints can be placed on the maximum and deglacial surface elevations at Mackay Glacier using surface-exposure dating (Chapter 7). The technique quantifies the time since a cobble or bedrock became exposed during thinning and retreat of a glacier, and has been applied to ice-free areas across Antarctica (e.g. Stone et al., 2003; Bentley et al., 2006; Mackintosh et al., 2007). To obtain a simple estimate of exposure time 
since last covered by ice, sampled rock surfaces must have no prior cosmogenic inheritance, and therefore, have had complete erosion of the surface during the last episode of glacial cover. Several important exposure dating considerations arise from the geomorphic assessment of Mackay Glacier. Firstly, the relict surfaces of a predominantly warm-based glacial regime have superimposed evidence of subsequent glacial cover and, therefore, bedrock samples would record a cumulative exposure signal from intermittent interglacials and glaciations. The bedrock surfaces at all sites, especially in areas of negligible erosion, would record an incorrect age of deglaciation since the LGM, reflecting cosmogenic inheritance from previously exposed episodes. Secondly, erratic cobbles within cryoturbated drift have likely experienced cycles of burial and exposure, and therefore would not provide reliable exposure age estimates. Thirdly, fresh erratics perched on bedrock surfaces were probably derived from subglacial locations, central to the trunk of Mackay Glacier, and therefore provide the best potential for determining the ice sheet history since the LGM.

This study highlights that small Transantarctic Mountain outlet glaciers can both erode and preserve bedrock surfaces during the same glacial episode, and that glacial material can experience reworking and redistribution. It highlights that a thorough assessment of the geomorphology is required to understand the spatial variability in past glacial regimes and, in turn, to obtain a reliable glacial history using surface-exposure dating. 


\section{Chapter 7}

\section{Rapid thinning of Mackay Glacier during stable Holocene climate}

\subsection{Introduction}

Observations suggest that the East Antarctic Ice Sheet (EAIS), the largest ice body on Earth locking up $53.3 \mathrm{~m}$ of sea level equivalent (Fretwell et al., 2013), is experiencing dynamic thinning and retreat of its outlet glaciers (Depoorter et al., 2013; Miles et al., 2013; Pritchard et al., 2009). Several ice-shelves, namely the Filchner, Amery, Shackleton and Totten Ice Shelves, buttress large areas of the EAIS that are grounded below sea level. This constitutes more than 2/3 of the total volume of Antarctic ice grounded on a bed below sea level, which is potentially vulnerable to 'Marine Ice Sheet Instability' (Fretwell et al., 2013; Weertman, 1974; Mercer, 1978; Schoof, 2007; Joughin et al., 2014; Mengel and Levermann, 2014; Favier et al., 2014). West Antarctica's Pine Island and Thwaites Glacier systems demonstrate significant, decadal-scale drawdown of ice in large catchments that occupy overdeepened basins (Johnson et al., 2014; Joughin et al., 2014; Favier et al., 2014; Rignot et al., 2011), thinning at rates up to $\sim 70-600 \mathrm{~cm} \mathrm{a}^{-1}$ (Payne et al., 2004; Pritchard et al., 2009).

Widespread ice-dynamic thinning initiated from perturbations at the bed and terminus (Dupont and Alley, 2005; Payne et al., 2004) may ultimately lead to irreversible mass loss if local topographic conditions favour retreat beyond a stability threshold (Mengel and Levermann, 2014). Currently, however, our ability to predict continental-scale consequences of such behaviour is hampered by the brevity of ice sheet observations (Miles et al., 2013; Pritchard et al., 2009; Shepherd et al., 2012). Constraints on the rate, duration and magnitude of past episodes of rapid ice sheet lowering are therefore urgently required.

Here, the style and mechanism of outlet glacier thinning is precisely reconstructed and demonstrated at Mackay Glacier, an outlet of the EAIS that flows through the Transantarctic Mountains (Figure 7.1). Using surface-exposure dating and glacier modelling, this work aims to answer several questions:

1. How thick was Mackay Glacier at the Last Glacial Maximum (LGM)? Is this quantitative estimate consistent with geomorphological interpretations? 
2. When did Mackay Glacier start thinning following the LGM? Did the glacier exhibit monotonic or non-linear surface lowering?

3. Was the timing and rate of thinning of Mackay Glacier concurrent with climatic and/or oceanic forcing, or was mass loss influenced by marine ice sheet instability?
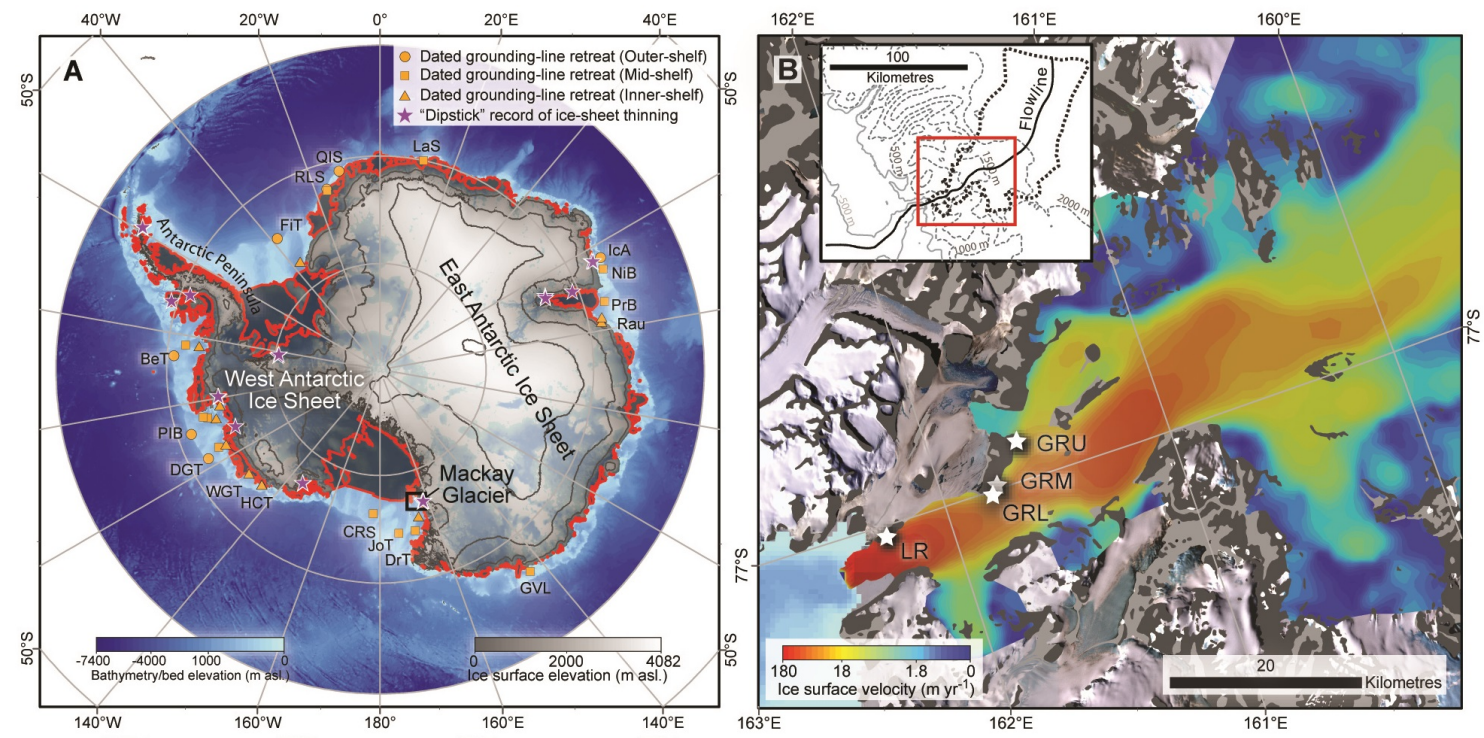

Figure 7.1 Geographic context of Mackay Glacier. A) Ice-shelves (red) buttress parts of Antarctic ice sheets that are grounded below sea level. Circum-Antarctic deglaciation records are labelled as in Figure 7.14. Mackay Glacier and the location of Figure 7.8 is marked by a black box. B) Surface velocity of present-day Mackay Glacier catchment (Rignot et al., 2011) with starred sample transect locations at Mt Suess/Gondola Ridge (upper, mid-lower, lower; GRU, GRM, GRL) and Low Ridge (LR), adjacent to the main flow path. Inset shows the flowline used in model simulations, and onshore surface and offshore bed contours.

\subsection{Geological evidence at Mackay Glacier}

Geomorphological evidence shows that Mackay Glacier was larger in the recent past (Chapter 6). Moraines at Cuff Cape, downstream of the present-day grounding-line, record small fluctuations of Mackay Glacier and adjacent New Glacier termini during the last two centuries (Möller, 1995). Prior to this, the glacial history is unconstrained, although regional-scale ice sheet reconstructions suggest that Mackay Glacier along with other outlets on the Victoria Land coast thickened at the LGM, due to buttressing by grounded ice in the Ross Sea (Denton and Hughes, 2000). The lower reaches of Mackay Glacier were targeted in order to determine for the first time its retreat history from the LGM to the present day. 


\subsubsection{Sample collection, age calculation and outliers}

Forty-four samples were collected for surface-exposure dating from two nunataks in the lower reaches of Mackay Glacier (Figure 7.1B). The upstream nunatak comprises Gondola Ridge, an elongate granitic ridge, and Mt Suess, a basaltic dome that peaks at 1,127 $\mathrm{m}$ asl. Present-day ice flow reaches velocities of $180 \mathrm{~m} \mathrm{a}^{-1}$ (Rignot et al., 2011) alongside this exposed bedrock, and the steep, ice-abraded flanks of Mt Suess and glacially smoothed and streamlined topography of Gondola Ridge provide evidence of thicker ice in the past. Quartz-rich sandstone and quartzite glacial erratics of the Beacon Supergroup can be found resting on both weathered and striated bedrock, and extend up to $890 \mathrm{~m}$ asl. on Mt Suess ( $\sim 340 \mathrm{~m}$ above the proximal modern ice surface). Low Ridge is an area of exposed granitic bedrock ( $\sim 205 \mathrm{~m}$ above the ice surface) that occurs downstream of the present-day grounding-line. Here, numerous sandstone cobbles are found on smooth, striated, granitic bedrock (Chapter 6).

"Dipstick" altitudinal transects have the potential to record past surface lowering of the glacier from a previously thicker configuration (e.g. Stone et al., 2003). Such sampling transects were carried out at Mt Suess/Gondola Ridge in order to constrain thickness at the LGM and subsequent initial thinning, and at Low Ridge to record more recent thinning near the present-day terminus (Figure 7.2). The Mt Suess/Gondola Ridge (upper) transect included 16 sampled erratics at 824-587 $\mathrm{m}$ asl (260-24 $\mathrm{m}$ above the modern ice surface), while an additional 18 samples were collected as part of two further transects at the downstream end of Gondola Ridge (mid-lower and lower). At Low Ridge, 10 samples were collected with an elevation range of 264-62 m asl (204-2 m above modern ice). To minimise the risk of sampling clasts containing an inherited cosmogenic inventory, erratics were sampled that showed signs of glacial transport and erosion, evident as facetted and abraded surfaces. Erratics that rested in locations unaffected by post-depositional processes were also prioritised in order to provide a simple exposure signal. Such samples were generally either perched on eroded bedrock or propped up by a thin layer $(1-3 \mathrm{~cm})$ of draped glacial till. Samples consisted of cobbles ( $\sim 10-25 \mathrm{~cm}$ length) and some small boulders $(<1 \mathrm{~m}$ across $)$ that were sub-sampled in the field. Details of samples collected are listed in Table 7.1.

Samples were prepared and beryllium was extracted as stated in the Methodology. Procedural blanks yielded mean ${ }^{10} \mathrm{Be} /{ }^{9} \mathrm{Be}$ ratios of $1.38 \times 10^{-15}$ with a standard deviation of $5.91 \times 10^{-16}$. Low measurable yields of a small number samples resulted in less precise exposure ages with $\sim 17-33 \%$ uncertainties (samples GR34, GR37, GR47, GR51, GR54, GR62b, GR64 and GR83). All samples were measured relative to the ETH Zurich in house standard $\mathrm{S} 2007 \mathrm{~N}$ (nominal ${ }^{10} \mathrm{Be} /{ }^{9} \mathrm{Be}$ ratio of $28.1 \pm 0.8 \times 10^{-12}$ ), which in turn was calibrated relative to the ICN $01-5-1$ standard $\left({ }^{10} \mathrm{Be} /{ }^{9} \mathrm{Be}\right.$ ratio of $\left.27.09 \pm 0.3 \times 10^{-12}\right)$ (Nishiizumi et al., 2007), and were corrected with procedural blanks. 


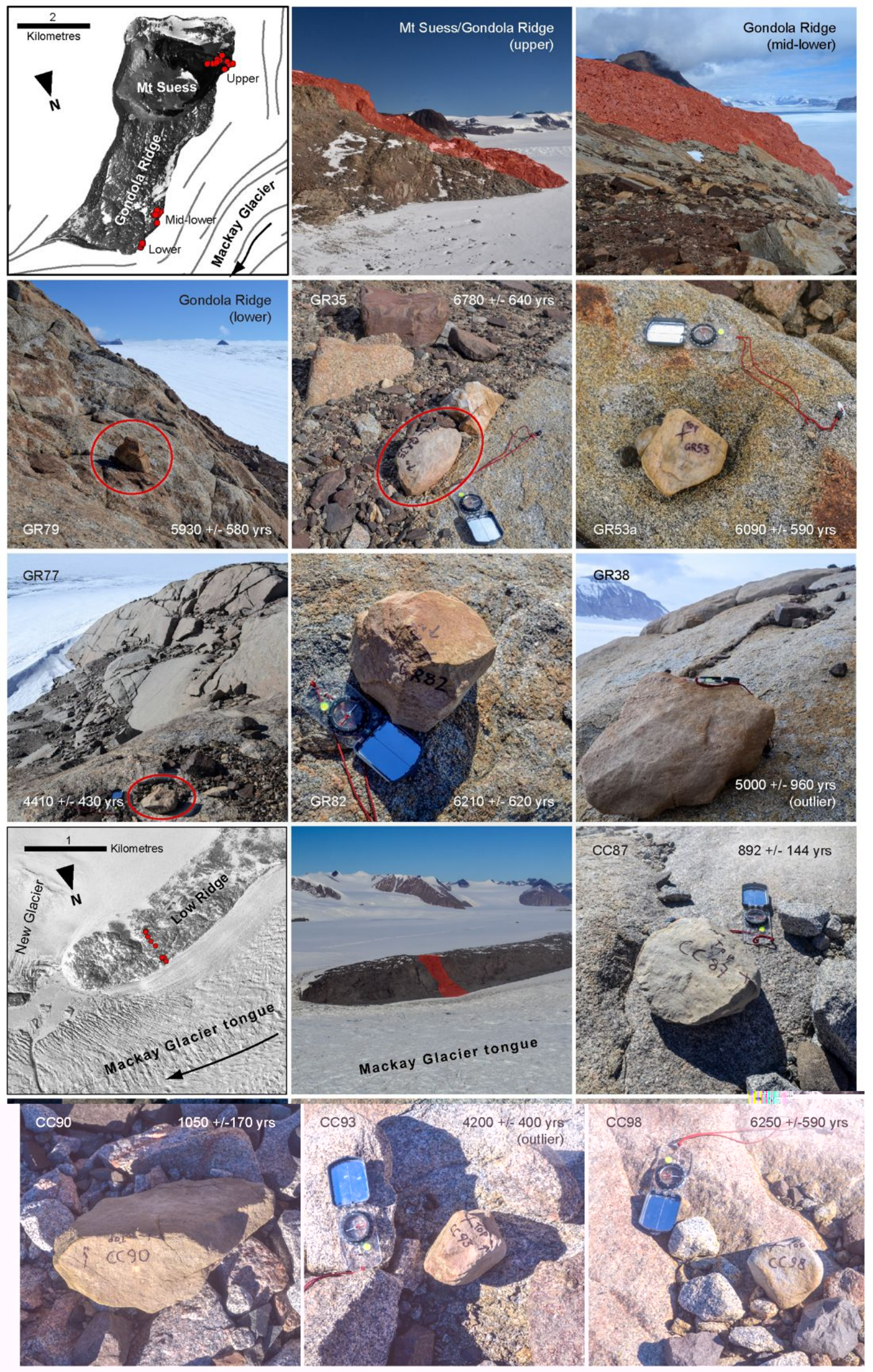

Figure 7.2 Locations of thinning transects and photos of example samples at Mackay Glacier. Mt Suess/Gondola Ridge transects extended 24-260 metres above the modern glacier surface, and included 16 (upper), 12 (mid-lower) and 6 (lower) sampled erratics. At Low Ridge, 10 samples were collected with an elevation range of 2-204 $\mathrm{m}$ above the ice surface. 
Table 7.1 Sample details and raw surface-exposure ages from Mackay Glacier, divided by altitudinal transect

\begin{tabular}{|c|c|c|c|c|c|c|c|c|c|c|c|c|c|c|c|c|c|c|c|c|c|c|c|c|c|c|}
\hline Sample ID & Latitude & Longitude & 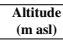 & $\begin{array}{l}\begin{array}{l}\text { Fereation above } \\
\text { modern ine }(m)\end{array} \\
\end{array}$ & $\begin{array}{c}\text { Sample } \\
\text { lithology }\end{array}$ & $\begin{array}{l}\text { Sample position } \\
\end{array}$ & $\begin{array}{c}\begin{array}{c}\text { Thichess } \\
\text { (cm) }\end{array} \\
\end{array}$ & $\begin{array}{l}\text { Shiedding } \\
\text { correction } \\
\end{array}$ & $\begin{array}{c}{ }^{\circ} \begin{array}{c}\text { Be carrier } \\
\text { (mg) }\end{array} \\
\text { (a) }\end{array}$ & ${ }^{1{ }^{10} \mathrm{Be} / \mathrm{Be}} \begin{array}{l}1 \sigma \\
(\%)\end{array}$ & 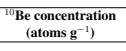 & $\begin{array}{l}1 \sigma \\
\text { (atoms } \\
\text { (1) }\end{array}$ & 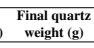 & 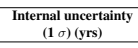 & $\begin{array}{l}\text { Lathlotom } \\
\text { Age (yrs) } \\
\end{array}$ & 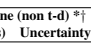 & $\begin{array}{l}\text { Desiters } \\
\text { ge (ys) }\end{array}$ & 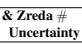 & 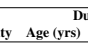 & 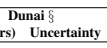 & $\begin{array}{l}\text { Litifor } \\
\text { Ige (ros) } \\
\end{array}$ & 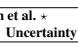 & $\begin{array}{l}\text { Lallsio } \\
\text { Age (yrs) }\end{array}$ & 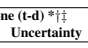 & 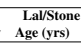 & $\begin{array}{l}\text { certaint } \\
\text { certex }\end{array}$ \\
\hline 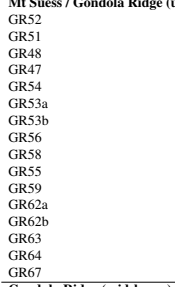 & 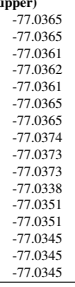 & 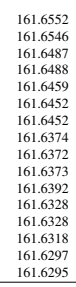 & $\begin{array}{l}824 \\
823 \\
795 \\
793 \\
778 \\
780 \\
780 \\
770 \\
710 \\
709 \\
648 \\
629 \\
629 \\
669 \\
588 \\
587\end{array}$ & $\begin{array}{l}2261 \\
220 \\
232 \\
230 \\
2219 \\
217 \\
217 \\
147 \\
147 \\
146 \\
85 \\
66 \\
66 \\
60 \\
25 \\
24 \\
24\end{array}$ & 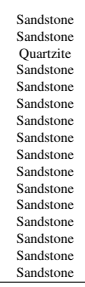 & 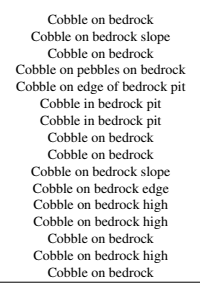 & $\begin{array}{l}2.75 \\
.550 \\
5.25 \\
4.25 \\
5.500 \\
4.00 \\
7.50 \\
4.50 \\
5.50 \\
5.00 \\
4.50 \\
4.25 \\
4.00 \\
3.350 \\
4.50 \\
5.50\end{array}$ & 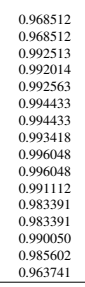 & 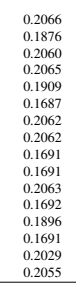 & 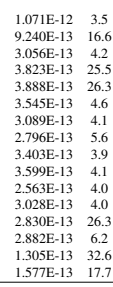 & 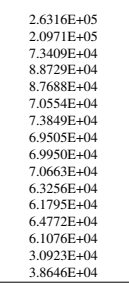 & 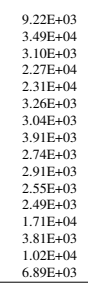 & 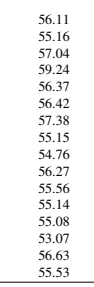 & 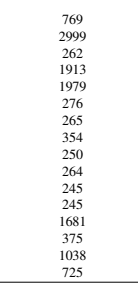 & 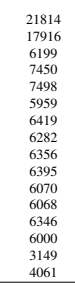 & 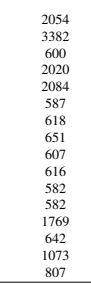 & 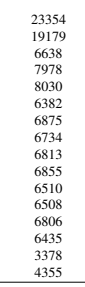 & 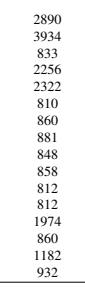 & 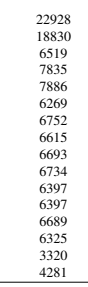 & $\begin{array}{l}2825 \\
3856 \\
815 \\
2213 \\
2279 \\
7929 \\
841 \\
863 \\
830 \\
839 \\
795 \\
795 \\
1939 \\
842 \\
1161 \\
915 \\
\end{array}$ & 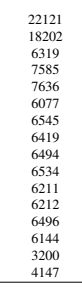 & $\begin{array}{l}2327 \\
2340 \\
6799 \\
2087 \\
2152 \\
663 \\
700 \\
700 \\
6000 \\
6099 \\
662 \\
662 \\
1836 \\
718 \\
1101 \\
846\end{array}$ & 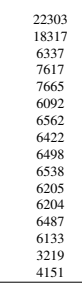 & 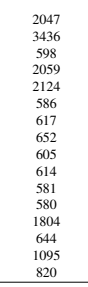 & 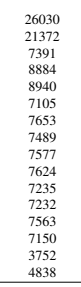 & 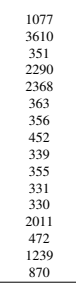 \\
\hline 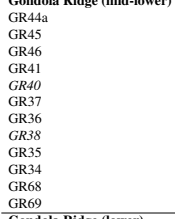 & 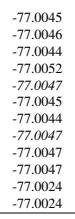 & 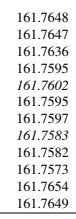 & $\begin{array}{l}569 \\
569 \\
563 \\
552 \\
539 \\
524 \\
524 \\
523 \\
512 \\
517 \\
505 \\
498\end{array}$ & $\begin{array}{l}97 \\
97 \\
90 \\
80 \\
66 \\
52 \\
52 \\
51 \\
49 \\
45 \\
43 \\
36 \\
36\end{array}$ & 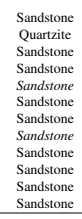 & 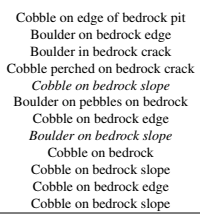 & $\begin{array}{l}3.75 \\
4.25 \\
3.300 \\
3.500 \\
5.00 \\
.450 \\
3.375 \\
5.00 \\
4.00 \\
5.00 \\
5.25 \\
5.75\end{array}$ & 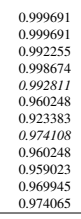 & $\begin{array}{l}0.01735 \\
0.11690 \\
0.01742 \\
0.01693 \\
0.01967 \\
0.1894 \\
0.01688 \\
0.01910 \\
0.1817 \\
0.01894 \\
0.1891 \\
0.1972\end{array}$ & 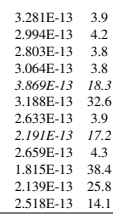 & 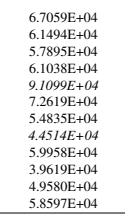 & 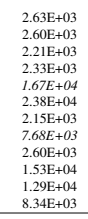 & $\begin{array}{l}56.49 \\
54.74 \\
56.07 \\
56.54 \\
55.53 \\
55.32 \\
53.89 \\
62.42 \\
53.55 \\
57.52 \\
54.17 \\
56.31\end{array}$ & $\begin{array}{l}2268 \\
2267 \\
2241 \\
1782 \\
2643 \\
2647 \\
8445 \\
288 \\
1722 \\
1453 \\
947 \\
\end{array}$ & $\begin{array}{l}6816 \\
6277 \\
5927 \\
6295 \\
9699 \\
8092 \\
62799 \\
48991 \\
6636 \\
6433 \\
5581 \\
6643\end{array}$ & $\begin{array}{l}651 \\
607 \\
553 \\
598 \\
1972 \\
2735 \\
599 \\
9965 \\
6645 \\
1765 \\
1532 \\
1109 \\
\end{array}$ & 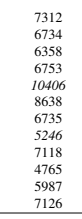 & $\begin{array}{l}910 \\
845 \\
789 \\
838 \\
2273 \\
83014 \\
838 \\
1098 \\
896 \\
1931 \\
1711 \\
1319 \\
\end{array}$ & 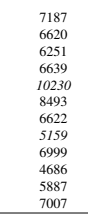 & $\begin{array}{l}891 \\
827 \\
773 \\
821 \\
2232 \\
2962 \\
821 \\
1078 \\
877 \\
1898 \\
1681 \\
1295\end{array}$ & 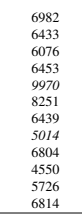 & $\begin{array}{l}742 \\
691 \\
6933 \\
6833 \\
2080 \\
2829 \\
689 \\
998 \\
734 \\
1130 \\
1594 \\
1181 \\
\end{array}$ & $\begin{array}{l}6967 \\
6471 \\
6059 \\
6435 \\
9915 \\
8231 \\
6418 \\
5000 \\
6783 \\
4541 \\
5705 \\
6791 \\
\end{array}$ & $\begin{array}{l}6699 \\
606 \\
562 \\
5065 \\
2005 \\
2790 \\
598 \\
962 \\
644 \\
1802 \\
1561 \\
1125 \\
\end{array}$ & 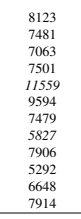 & $\begin{array}{l}364 \\
355 \\
310 \\
330 \\
2239 \\
3157 \\
335 \\
1015 \\
383 \\
2055 \\
1737 \\
1141 \\
\end{array}$ \\
\hline 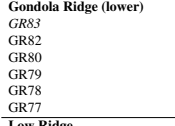 & 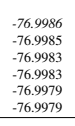 & $\begin{array}{l}1161.7880 \\
116.7878 \\
1161.7890 \\
116.717900 \\
161.7904 \\
161.994\end{array}$ & $\begin{array}{l}449 \\
446 \\
430 \\
429 \\
417 \\
416 \\
\end{array}$ & $\begin{array}{l}44 \\
41 \\
25 \\
24 \\
12 \\
11 \\
11\end{array}$ & 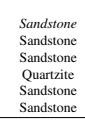 & 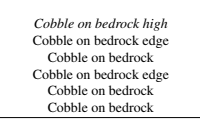 & $\begin{array}{l}7.50 \\
3.00 \\
4.00 \\
6.00 \\
4.25 \\
4.50 \\
\end{array}$ & 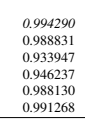 & $\begin{array}{l}0.20257 \\
0.1695 \\
0.0697 \\
0.01745 \\
0.1735 \\
0.1737 \\
\end{array}$ & 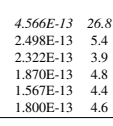 & 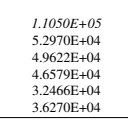 & 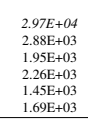 & $\begin{array}{l}56.63 \\
53.11 \\
52.74 \\
46.47 \\
55.46 \\
57.16\end{array}$ & $\begin{array}{l}3323 \\
330 \\
2422 \\
2822 \\
172 \\
201 \\
\end{array}$ & $\begin{array}{l}13050 \\
6070 \\
6151 \\
5502 \\
3865 \\
4315 \\
4315\end{array}$ & $\begin{array}{l}3702 \\
6237 \\
578 \\
5778 \\
426\end{array}$ & $\begin{array}{l}13996 \\
6510 \\
65994 \\
6220 \\
4443 \\
4626 \\
\end{array}$ & $\begin{array}{l}426 \\
847 \\
821 \\
7523 \\
587 \\
587\end{array}$ & $\begin{array}{l}13764 \\
64636 \\
64866 \\
61118 \\
4075 \\
4550 \\
450\end{array}$ & $\begin{array}{l}4855 \\
830 \\
804 \\
778 \\
512 \\
575 \\
\end{array}$ & $\begin{array}{l}13445 \\
6232 \\
6354 \\
59565 \\
3952 \\
44222\end{array}$ & $\begin{array}{l}3857 \\
7731 \\
6671 \\
6458 \\
482 \\
482 \\
\end{array}$ & $\begin{array}{l}13340 \\
62025 \\
2028 \\
5930 \\
3950 \\
4411 \\
4411\end{array}$ & $\begin{array}{l}3374 \\
623 \\
586 \\
577 \\
377 \\
425 \\
\end{array}$ & $\begin{array}{l}15550 \\
77232 \\
7324 \\
6908 \\
4603 \\
5139 \\
5139\end{array}$ & $\begin{array}{l}4214 \\
423 \\
329 \\
367 \\
228 \\
263 \\
\end{array}$ \\
\hline 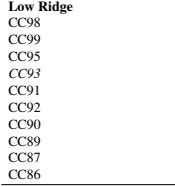 & 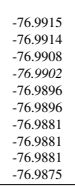 & 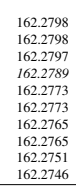 & $\begin{array}{l}264 \\
263 \\
245 \\
219 \\
179 \\
179 \\
87 \\
86 \\
84 \\
62 \\
\end{array}$ & $\begin{array}{l}203 \\
183 \\
1159 \\
119 \\
119 \\
27 \\
26 \\
24 \\
24 \\
\end{array}$ & 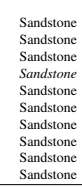 & 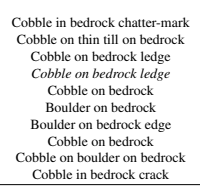 & $\begin{array}{l}4.95 \\
3.50 \\
43.50 \\
3.25 \\
3.25 \\
4.50 \\
4.50 \\
5.75 \\
3.50 \\
3.50\end{array}$ & 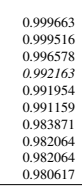 & 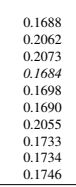 & 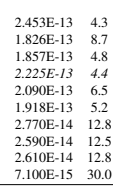 & 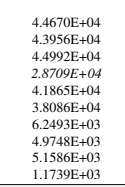 & 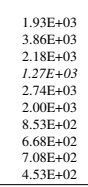 & 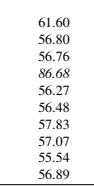 & 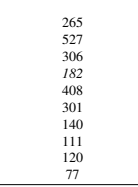 & 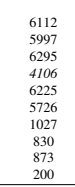 & $\begin{array}{l}344 \\
741 \\
627 \\
401 \\
678 \\
582 \\
166 \\
133 \\
142 \\
79 \\
\end{array}$ & 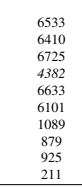 & $\begin{array}{l}823 \\
943 \\
8593 \\
5893 \\
8989 \\
799 \\
1197 \\
1177 \\
85 \\
\end{array}$ & 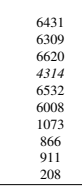 & $\begin{array}{l}806 \\
9266 \\
842 \\
542 \\
879 \\
774 \\
1193 \\
154 \\
165 \\
84\end{array}$ & 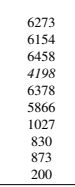 & $\begin{array}{l}818 \\
813 \\
710 \\
454 \\
756 \\
656 \\
173 \\
138 \\
148 \\
80 \\
\end{array}$ & 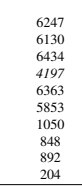 & 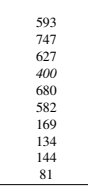 & 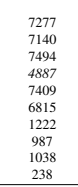 & $\begin{array}{l}352 \\
646 \\
3298 \\
241 \\
587 \\
317 \\
139 \\
144 \\
92 \\
92\end{array}$ \\
\hline
\end{tabular}

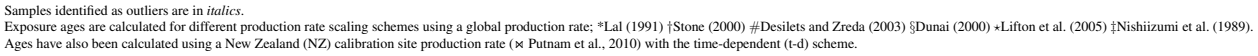


Table 7.2 Bayesian modelled surface-exposure ages at Mackay Glacier, divided by altitudinal transect

\begin{tabular}{|c|c|c|c|c|c|c|c|c|c|c|}
\hline Sample ID & $\begin{array}{c}\text { Altitude } \\
\text { ( } \mathrm{m} \text { asl.) }\end{array}$ & $\begin{array}{l}\text { Elevation above } \\
\text { modern ice (m) }\end{array}$ & $\begin{array}{c}\text { Weighted mean } \\
\text { (Lal/Stone t-d) (yrs) }\end{array}$ & $\begin{array}{c}\text { Standard } \\
\text { error }\end{array}$ & $\begin{array}{c}\text { Weighted mean } \\
\text { (Lal/Stone t-d, NZ) (yrs) } \\
\end{array}$ & $\begin{array}{c}\text { Standard } \\
\text { error }\end{array}$ & $\begin{array}{l}\text { Age-elevation modelled } \\
\text { (Lal/Stone t-d) (yrs) }\end{array}$ & $\begin{array}{l}\text { Modelled error } \\
(1 \sigma)(\mathbf{y r s})\end{array}$ & $\begin{array}{l}\text { Age-elevation modelled } \\
\text { (Lal/Stone t-d, NZ) (yrs) }\end{array}$ & $\begin{array}{c}\text { Modelled error } \\
(1 \sigma)(\mathbf{y r s})\end{array}$ \\
\hline \multicolumn{11}{|c|}{ Mt Suess / Gondola Ridge (upper) } \\
\hline GR52 & 824 & 261 & \multirow{5}{*}{6437} & \multirow{5}{*}{574} & \multirow{5}{*}{7425} & \multirow{5}{*}{347} & \multirow{4}{*}{$\begin{array}{l}22303 \\
18317\end{array}$} & \multirow{2}{*}{$\begin{array}{l}2047 \\
3436\end{array}$} & \multirow{2}{*}{$\begin{array}{l}26030 \\
21372\end{array}$} & \multirow{2}{*}{$\begin{array}{l}1077 \\
3610\end{array}$} \\
\hline GR51 & 823 & 260 & & & & & & & & \\
\hline GR48 & 795 & 232 & & & & & & 275 & 7657 & 286 \\
\hline GR47 & 793 & 230 & & & & & & 215 & $163 \%$ & 286 \\
\hline GR54 & 782 & 219 & & & & & \multirow{3}{*}{6467} & \multirow{3}{*}{224} & \multirow{3}{*}{7555} & \multirow{3}{*}{174} \\
\hline GR53a & 780 & 217 & \multirow[t]{3}{*}{6367} & \multirow[t]{2}{*}{417} & \multirow[t]{2}{*}{7402} & \multirow[t]{2}{*}{253} & & & & \\
\hline GR53b & 780 & 217 & & & & & & & & \\
\hline GR56 & 710 & 147 & & \multirow{3}{*}{360} & \multirow{3}{*}{7574} & \multirow{3}{*}{216} & \multirow{3}{*}{6377} & \multirow{3}{*}{207} & \multirow{3}{*}{7481} & \multirow{3}{*}{139} \\
\hline GR58 & 710 & 147 & \multirow[t]{2}{*}{6489} & & & & & & & \\
\hline GR55 & 709 & 146 & & & & & & & & \\
\hline GR59 & 648 & 85 & \multirow{4}{*}{6231} & \multirow{4}{*}{552} & \multirow{4}{*}{7241} & & 6278 & 221 & 7416 & 129 \\
\hline GR62a & 629 & 66 & & & & 326 & & 253 & 7342 & 141 \\
\hline GR62b & 629 & $\begin{array}{l}66 \\
50\end{array}$ & & & & & 6067 & 5 & 1072 & 141 \\
\hline GR63 & 613 & 50 & & & & & 6067 & 315 & 7265 & 168 \\
\hline $\begin{array}{l}\text { GR64 } \\
\text { GR67 }\end{array}$ & $\begin{array}{l}588 \\
587\end{array}$ & $\begin{array}{l}25 \\
24\end{array}$ & 3816 & 656 & 4479 & 712 & 3816 & 656 & 4479 & 712 \\
\hline Gondola Ri & gie (lower) & & & & & & & & & \\
\hline GR82 & 446 & 41 & & & & & 6421 & 439 & 7357 & 282 \\
\hline GR80 & 430 & 25 & 6106 & 411 & 7139 & 245 & 6011 & 363 & 7099 & 220 \\
\hline GR79 & 429 & 24 & & & & & & & & \\
\hline $\begin{array}{l}\text { GR78 } \\
\text { GR77 }\end{array}$ & $\begin{array}{l}417 \\
416\end{array}$ & $\begin{array}{l}12 \\
11\end{array}$ & 4153 & 282 & 4833 & 172 & 4153 & 442 & 4833 & 172 \\
\hline Low Ridge & & & & & & & & & & \\
\hline CC98 & 264 & 204 & 6202 & 464 & 7246 & 309 & 6414 & 339 & 7384 & 231 \\
\hline CC99 & 263 & 203 & 0202 & & 1246 & & & & & \\
\hline CC95 & 245 & 185 & & & & & 6215 & 311 & 7241 & 211 \\
\hline CC91 & 179 & 119 & 6069 & 442 & 7031 & 309 & 5988 & 336 & 7047 & 239 \\
\hline CC92 & 179 & 119 & & & & & & & & \\
\hline $\begin{array}{l}\text { CC90 } \\
\text { CC89 }\end{array}$ & $\begin{array}{l}87 \\
87\end{array}$ & $\begin{array}{l}27 \\
26\end{array}$ & & & & 85 & & & 1051 & \\
\hline CC89 & $\begin{array}{l}86 \\
84\end{array}$ & $\begin{array}{l}26 \\
24\end{array}$ & 914 & 80 & 1064 & 0. & 899 & 80 & 1031 & 86 \\
\hline CC87 & $\begin{array}{l}84 \\
62\end{array}$ & $\begin{array}{c}24 \\
2\end{array}$ & & & & & 218 & 81 & 253 & 93 \\
\hline
\end{tabular}

Weighted mean ages were calculated for cobbles at equal elevations, where samples were collected within 2 metres of each other and whose internal uncertainties were overlapping. Exposure ages derived from Bayesian age-elevation modelling are considered best estimates. 
Surface-exposure ages were calculated from the measured concentrations of ${ }^{10} \mathrm{Be}$, corrected for topographic shielding, sample thickness, quartz density $\left(2.7 \mathrm{~g} \mathrm{~cm}^{3}\right)$ and an Antarctic atmospheric pressure gradient, using CRONUS-Earth online calculator (Balco et al., 2008) (Table 7.1). The production rate of ${ }^{10} \mathrm{Be}$ is currently not well constrained in Antarctica, and production rates from both a global dataset (Balco et al., 2008) (production due to spallation of $4.49 \pm 0.39$ to $4.96 \pm 0.43$ atoms $\mathrm{g} \mathrm{a}^{-1}$ ) and New Zealand (NZ) calibration site (Putnam et al., 2010) (3.84 \pm 0.08 atoms $\mathrm{g} \mathrm{a}^{-1}$ ) have been applied to Antarctic chronologies in the past (Stone et al., 2003; Johnson et al., 2014). The global production rate was prioritised in this study as the high-precision $\mathrm{NZ}$ production rate (44 $\left.{ }^{\circ} \mathrm{S}\right)$ would produce ages with artificially low uncertainties at Mackay Glacier $\left(77{ }^{\circ} \mathrm{S}\right)$, given the uncertain temporal and spatial scaling. The choice of production rate does not significantly affect the reconstructed thinning rate at Mackay Glacier, and instead primarily influences its absolute timing.

Across all 4 transects, 44 exposure ages ranged from the LGM to recent pre-industrial ( $22.3 \pm 2$ ka to $245 \pm 75$ years) (Figure 7.3). Only four sample outliers were identified in the complete dataset, by their stratigraphic positions within and between transects. On Gondola Ridge (mid-lower), two samples were erroneously old or young (GR38, GR40), representing micro-inheritance and some post-depositional effects, respectively. At Gondola Ridge (lower), the oldest sample $(13,080 \pm 3700$, GR83) is not consistent with the thinning trends recorded in adjacent transects; if the surface elevation here was $<50 \mathrm{~m}$ above present before $\sim 13 \mathrm{ka}$ then an unfeasible ice surface profile would exist with sites upstream and downstream, which record glacier surfaces $>200 \mathrm{~m}$ higher at this time. For this reason, Sample GR83 most likely contains a small amount of inheritance. A single sample on Low Ridge (CC93) is not consistent with exposure ages above and below its elevation. This minor outlier has probably been affected by post-depositional rotation or spalling. The remaining 40 samples provide a direct constraint on outlet glacier thinning from the LGM to pre-industrial times.

\subsubsection{Bayesian age-elevation modelling}

The high coherence and density of the cosmogenic exposure ages allows the timing of former ice surface lowering at Mackay Glacier to be refined between the LGM and present day. Firstly, at elevations where internal uncertainties overlapped, a weighted mean and standard error was calculated. Secondly, age-elevation modelling was used to reduce the possible age range of exposure at each sampled elevation within the transects.

Several age-depth models have been developed to improve the age distributions that in 


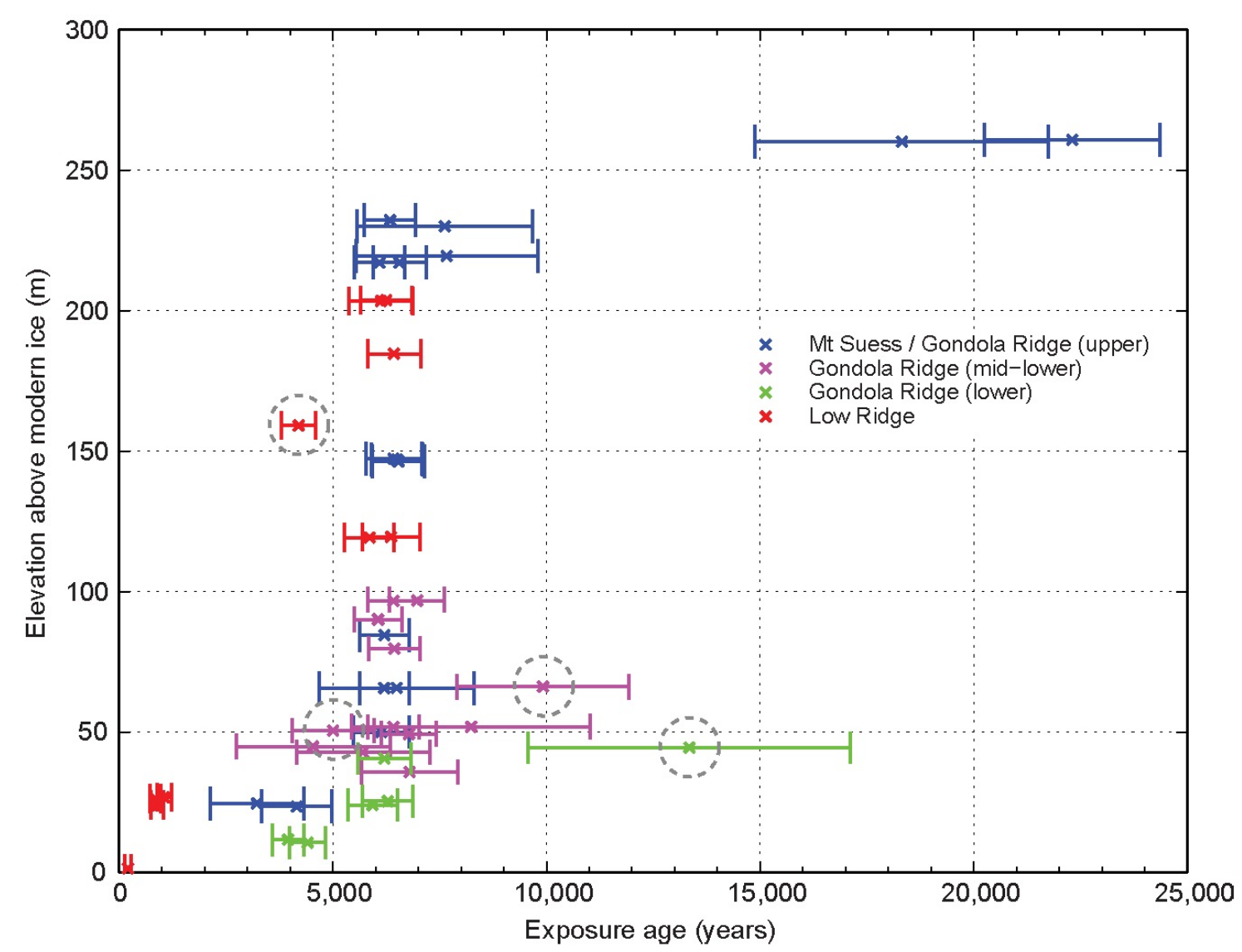

Figure 7.3 Surface exposures ages from 4 transects at Mackay Glacier. Displayed are the raw, unmodelled ages $(1 \sigma)$ with outliers included and identified (dashed circles). Surface lowering is recorded from $\sim 22$ ka to 200 years ago with an episode of rapid thinning evident in all 4 transects.

part result from calibrated radiocarbon dates in depositional settings (e.g. Grimm, 1990; Bennett, 1992; Ramsey, 1998; Heegaard et al., 2005; Blaauw et al., 2011). These models primarily use the respective age distribution and stratigraphic position of samples, and can be easily adapted for application to altitudinal transects that record ice surface lowering. In this manner, I applied the 'OxCal' Bayesian age-depth model (Ramsey, 1998, 2008) to the Mackay Glacier dataset, with the assumption that older and higher samples would be exposed by glacier thinning before the lower samples. This particular model uses Prior information (e.g. elevation, order of exposure) and Likelihood information (probability density functions of the age distribution for each sample elevation). Markov Chain Monte Carlo sampling is performed on these inputs, using the Metropolis-Hastings algorithm to sample possible solutions (Gilks et al., 1996). The resulting modelled ages are Posterior probability densities for each sampled elevation.

As I intend to compare the output age ranges to other chronologies, external exposure age uncertainties that represent production rate uncertainty from spallation and muon capture 
were used (Balco et al., 2008). Although the Gondola Ridge (mid-lower) chronology is consistent with other transects, it was not possible to apply Bayesian age-elevation modelling at this site; the OxCal model identified too much stratigraphic scatter of ages within the transect (Ramsey, 2008). However, at Mt Suess/Gondola Ridge (upper), Gondola Ridge (lower) and Low Ridge, this statistical analysis of high-density sample transects has allowed the uncertainty of raw exposure ages to be considerably reduced (Figure 7.4; Table 7.2).

\subsubsection{Record of surface lowering at Mackay Glacier}

The Mackay Glacier chronology provides a near-complete record of ice thinning from the LGM to present, revealing initially gradual surface lowering, punctuated by an episode of rapid thinning during the Holocene (Figure 7.4). Un-dated glacial erratics occur on Mt Suess up to $340 \mathrm{~m}$ above the modern ice surface ( $~ 890 \mathrm{~m}$ asl), indicating that Mackay Glacier may have been this thick during the LGM, or at another time in the recent geological past. Two dated samples from a surface elevation $\sim 260 \mathrm{~m}$ above the modern glacier date to the LGM $(\sim 22.3 \pm 2 \mathrm{ka}$ and $18.3 \pm 3.5 \mathrm{ka})$, however, as their internal uncertainties do not overlap, the maximum LGM thickness and exact timing of initial deglaciation is uncertain. Subsequent ice surface lowering below this elevation appears to have been initially gradual, with just $\sim 30 \mathrm{~m}$ of thinning occurring before the early Holocene. However, this part of the record lacks sufficient sample coverage to clarify surface elevation changes that might have occurred between the LGM and early Holocene, for example during Meltwater Pulse 1a (Deschamps et al., 2012) and, therefore, an episode of thinning and then thickening prior to the Holocene cannot be ruled out.

During the early/mid Holocene, rapid thinning is recorded by 28 age-modelled samples in all transects. The onset of this thinning occurred at $7.8-6.8 \mathrm{ka}$, with the age range dependent on the ${ }^{10} \mathrm{Be}$ production rate used (Figure 7.5). This event is best preserved in the Mt Suess/Gondola Ridge (upper) transect, where rapid thinning is recorded from 230 to $50 \mathrm{~m}$ above the modern ice surface ( $180 \mathrm{~m}$ total lowering), which is $>80 \%$ of the total LGM-to-present thinning. The end of rapid thinning is likely recorded from 5 exposure ages at Gondola Ridge (lower) as a change to gradual surface lowering. Data from Low Ridge show that this more gradual thinning (a further $\sim 25 \mathrm{~m}$ of ice surface lowering) persisted until $\sim 250$ years ago, at least in the lower reaches of the glacier. 

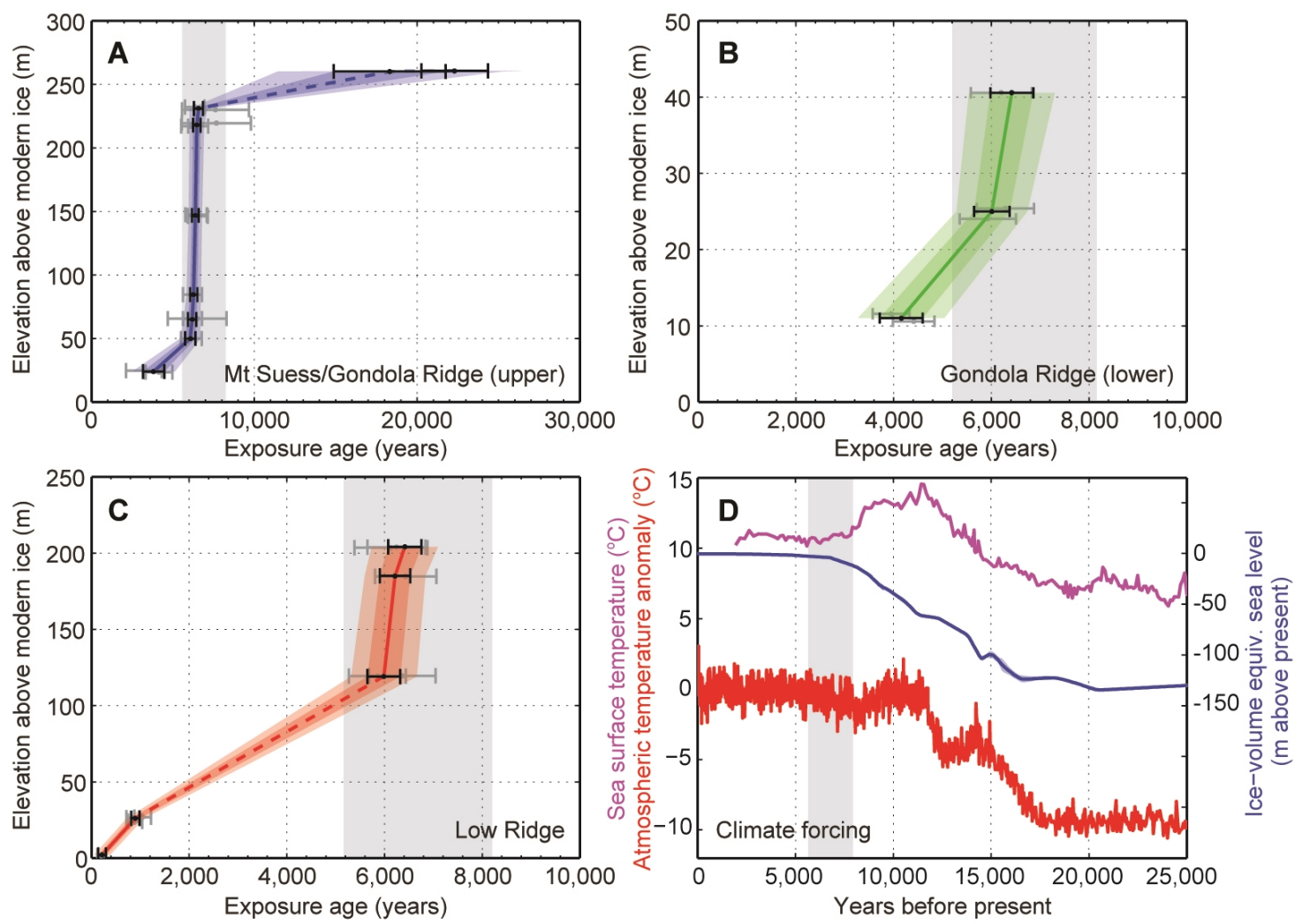

Figure 7.4 Age-modelled chronology of surface lowering at Mackay Glacier and regional climate forcing. Bayesian age-elevation modelled surface-exposure ages (black,

$1 \sigma$ ) are shown for 3 of 4 transects (A-C), linearly interpolated with 1 and $2 \sigma$ uncertainty. Initial raw exposure ages are in grey. Rapid thinning is recorded at $\sim 6.8-6.0$

$\mathrm{ka}$. This episode does not correspond to significant increases in regional sea surface temperature (Pahnke and Sachs, 2006), atmospheric temperature (Jouzel et al., 2007) or sea level (Lambeck et al., 2014) (D), irrespective of exposure age uncertainty (grey shaded area; Fig. 7.5).

\subsubsection{Regression analysis of rapid thinning}

In order to estimate the rate and duration of rapid thinning at Mackay Glacier, linear regression analysis was carried out on data from the Mt Suess/Gondola Ridge (upper) and Low Ridge transects for the period of $\sim 6.8-6.0 \mathrm{ka}$. Error-weighted least-squares regression was applied randomly to normally-distributed exposure ages $(2 \sigma)$ through a 4000-iteration Monte Carlo simulation (Figure 7.6). Rates and durations of rapid thinning were estimated from the distribution of feasible, positive-sloping linear regressions, with uncertainty generally reflective of the number of samples contributing to each transect and their respective uncertainties.

Firstly, the rate of thinning was assessed, and while it may have varied during this time period, this regression approach extracts a linear estimate implied by the samples (Figure 7.7A). Previous authors used raw exposure ages ( $1 \sigma$ internal uncertainty) for regression analysis to derive thinning rates (Johnson et al., 2014). Applied to Low Ridge, regression 

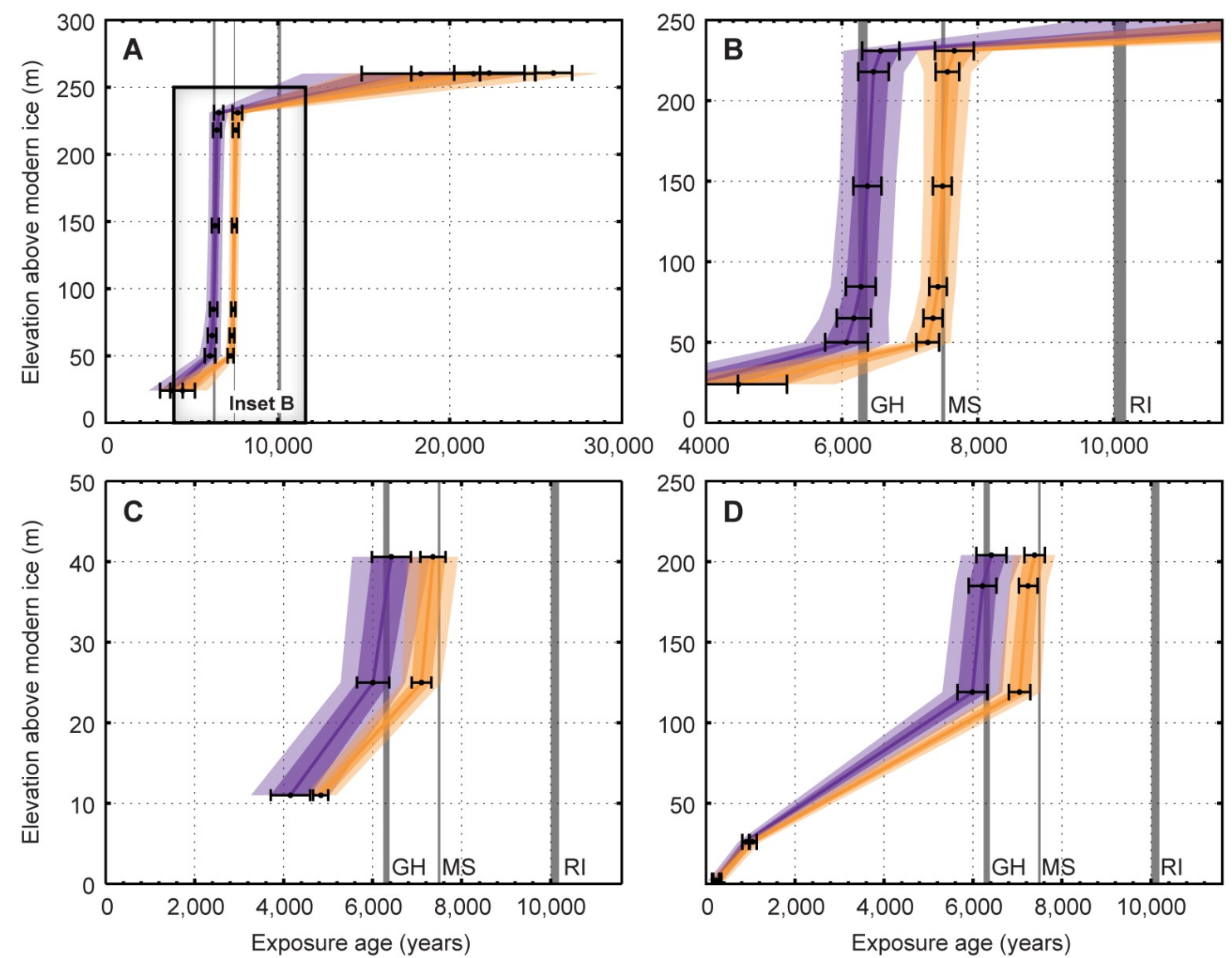

Figure 7.5 Recorded thinning of Mackay Glacier from differing ${ }^{10} \mathrm{Be}$ production rates, shown for the 3 most coherent transects (A and B, Mt Suess/Gondola Ridge upper; C, Gondola Ridge lower; D, Low Ridge). Displayed are Bayesian age-elevation modelled ages $(1 \sigma)$, linearly interpolated between sample elevations ( 1 and $2 \sigma)$. The ages were initially calculated with both the global production rate (Balco et al., 2008) (purple) and New Zealand calibration site production rate (Putnam et al., 2010) (orange), which can be considered as probable minimum and maximum estimations of the absolute ages.

Vertical grey bars denote minimum age markers of grounded ice retreat north of Ross Island (RI), in McMurdo Sound (MS) and of ice-shelf removal in Granite Harbour (GH), discussed in the text.

analysis indicates thinning at $8.2-358.8 \mathrm{~cm} \mathrm{a}^{-1}(2 \sigma)$. Due to the higher sample density at Mt Suess/Gondola Ridge (upper, $n=6$ ), I use the Bayesian-modelled ages to estimate thinning rates, which are considered to be the most reliable surface lowering constraints. Here, regression analysis records thinning of 33.1-80.2 $\mathrm{cm} \mathrm{a}^{-1}(2 \sigma)$ and it is likely that the upper end of this range better represents the peak of thinning during this period.

Secondly, the duration of this rapid thinning episode was calculated, based on the start and end points of the modelled regressions (Figure 7.7B). At Mt Suess/Gondola Ridge (upper), the duration of this event is constrained by a dominant, normally-distributed peak at $\sim 420$ years, which also corresponds to its median and mean values. At Low Ridge, a wide and skewed distribution of possible durations are estimated with a best fit of $\sim 300$ 

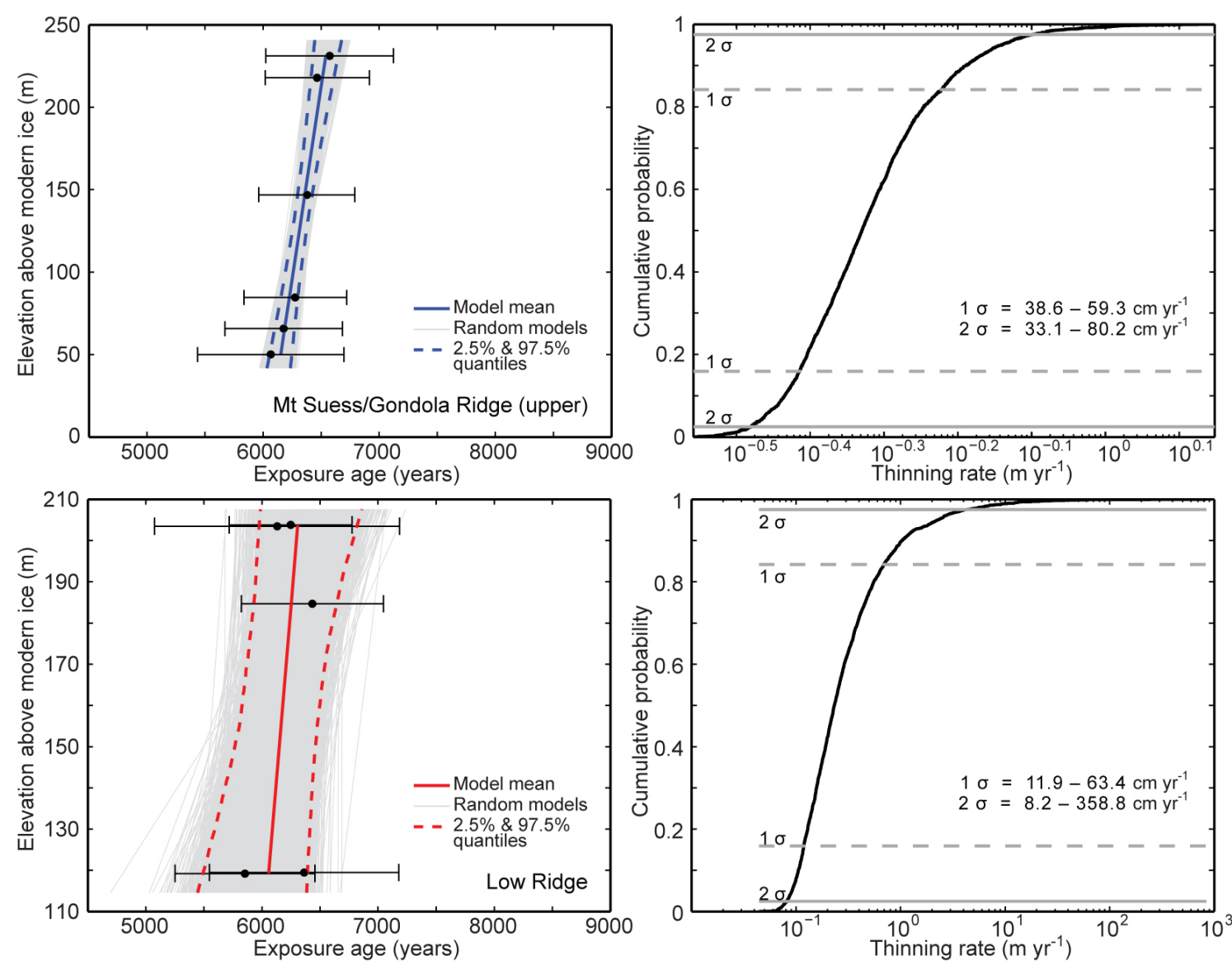

Figure 7.6 Regression analysis of rapid thinning at Mt Suess/Gondola Ridge (upper) and Low Ridge. Left panels show exposure ages $(2 \sigma)$ and modelled thinning profiles generated from Monte Carlo regression analysis for each transect respectively. Right panels show the corresponding thinning rate probability distributions. Thinning at $\mathrm{Mt}$ Suess/Gondola Ridge (upper) was estimated using Bayesian age-modelled ages and uncertainties, while at Low Ridge raw ages were alternatively used in regression analysis.

years, however, the median value is consistent within $1 \sigma$ of Mt Suess/Gondola Ridge (upper) at $\sim 400$ years. These are considered to be minimum estimates as the onset of rapid thinning is not recorded at Mt Suess/Gondola Ridge (upper), while neither the start or end is recorded at Low Ridge.

In summary, Mackay Glacier thinned at a rate during the Holocene that is similar to that observed today at rapidly changing outlet glaciers in Antarctica such as Totten and Pine Island Glaciers (Pritchard et al., 2009). At Mackay Glacier, this episode of rapid thinning likely persisted for at least $\sim 420$ years, far beyond the period of modern satellite observations. 

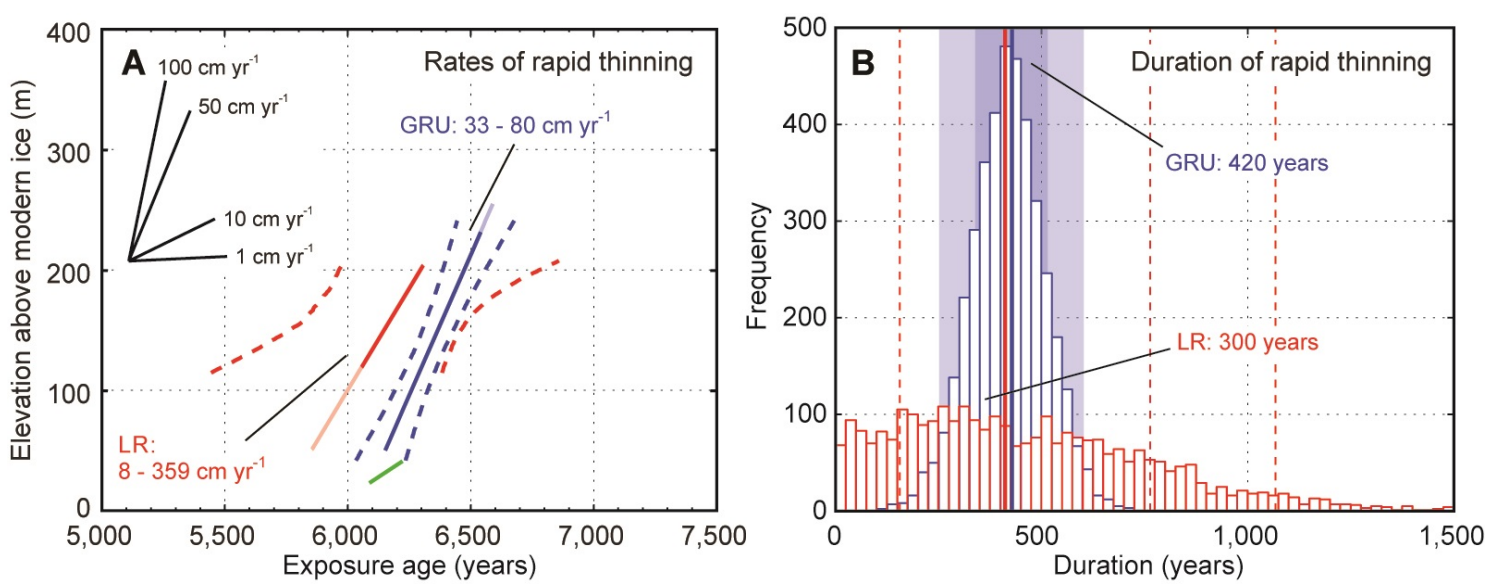

Figure 7.7 Rates and durations of rapid thinning derived from the surface-exposure chronology. A) Rapid surface lowering is estimated at Mt Suess/Gondola Ridge (upper) (GRU) and Low Ridge (LR), assuming linear thinning rates between $\sim 6.8$ and $6.0 \mathrm{ka}$. GRU provides a tighter estimate of $33.1-80.2 \mathrm{~cm} \mathrm{a}^{-1}$. The best fit (solid lines) and $95 \%$ confidence bounds (dashed lines) are shown, as in Figure 7.6. At Low Ridge, the full extent of rapid thinning is uncertain, however the end of this episode and a change to more gradual thinning may be recorded at Gondola Ridge (lower) (green line). B) Rapid thinning lasted for $\sim 420$ years, based on the higher quality GRU chronology. As the onset of rapid thinning here may have been from the LGM surface elevation, this is considered to be a minimum estimate of duration. Median estimates are shown by solid lines, while 1 and $2 \sigma$ are denoted by a shaded area (GRU) and dashed lines (LR).

\subsection{Stable environmental forcing during the Holocene}

Substantial, rapid and prolonged surface lowering at Mackay Glacier occurred during a period of stable climate similar to that of today (Figure 7.4D). Atmospheric temperature reached near-present values by $\sim 10-12 \mathrm{ka}$ and may have cooled slightly during the Holocene (Jouzel et al., 2007), ruling out enhanced surface melt as the mechanism that forced retreat and associated thinning. Rates of eustatic sea level rise reduced significantly after $\sim 8 \mathrm{ka}$ (Lambeck et al., 2014) and regional isostatic uplift would have further reduced or even reversed the effects of sea level rise during this time (Peltier, 2004).

Warming ocean water, and in particular, increased submarine melt from an influx of Circumpolar Deep Water, most likely forced ice sheet retreat following the LGM (Golledge et al., 2014b). However, there is no evidence of substantial additional ocean forcing at the time of rapid surface lowering that is recorded in the chronology; sea surface temperatures peaked prior to $10 \mathrm{ka}$ in the southwest Pacific Ocean (Pahnke and Sachs, 2006). Therefore, external forcing from the atmosphere or ocean alone cannot explain the rapid nature of recorded ice surface lowering. 


\subsection{Regional context for deglaciation as a potential cause of rapid thinning}

\subsubsection{Deglaciation in the western Ross Sea}

Rapid glacier thinning can result from positive feedbacks following perturbations near the terminus (Schoof, 2007; Dupont and Alley, 2005), and it is therefore necessary to investigate deglaciation in the vicinity of Mackay Glacier at the time of rapid thinning. Multiple onshore and offshore records constrain the timing and extent of past ice retreat in the western Ross Sea (Table 7.3; Figure 7.8). For comparison between this regional evidence and the thinning transects at Mackay Glacier, all ${ }^{14} \mathrm{C}$ (radiocarbon) dates have been corrected for marine reservoir effects and calibrated using the marine calibration curve (Marine09) as recommended by their original sources and a recent review of regional deglaciation (Anderson et al., 2014). Moreover, dates obtained from offshore cores were only included if they showed linear sedimentation rates and consistent ${ }^{13} \mathrm{C}$ values through the core (Domack et al., 1999).

Based on these dates, grounded ice and an ice-shelf front in the Ross Sea likely retreated to just north of Ross Island by $\sim 10 \mathrm{ka}$ (Mckay et al., 2008), or slightly earlier if a regional reservoir correction (e.g. 1,200-1,300 years) is used instead of the core's larger surface ${ }^{14} \mathrm{C}$ correction. At $\sim 9.4 \mathrm{ka}$, Explorer's Cove was still occupied by grounded ice (Hall and Denton, 2000) and Hall et al. (2004) suggest grounded ice in McMurdo Sound had yet to retreat. A relative sea level curve produced from radiocarbon dating of raised beach deposits indicates final unloading of grounded ice adjacent to the Scott Coast occurred at $\sim 7.5 \mathrm{ka}$ (Hall et al., 2004), which is supported by a radiocarbon dated bivalve in a sediment core collected off Cape Bird (Licht et al., 1996). The remaining ice-shelf disappeared between $\sim 7.5 \mathrm{ka}$ and $\sim 6.3 \mathrm{ka}$, at which point Granite Harbour (Domack et al., 1999), Gneiss Point and Marble Point (Hall and Denton, 1999, 2000) had become open water. An ice-shelf may have however still existed immediately south at Explorer's Cove (Hall and Denton, 2000).

The geomorphology on the present-day seabed helps infer the style and rate of past grounded ice retreat in and around McMurdo Sound during the Holocene (Greenwood et al., 2012). Immediately downstream of Mackay Glacier's terminus is the deep Mackay Sea Valley (below $-800 \mathrm{~m}$ asl), which shallows and widens into a trough and then the southern Drygalski Basin, north of McMurdo Sound (Figure 7.9). A series of groundingzone wedges (GZWs) preserved in the outer part of the trough imply that the initial retreat of the Mackay Glacier grounding-line was most likely staggered. Although data are not available to constrain the timing and duration of these stillstands, studies of similar-sized 
Table 7.3 Deglaciation evidence in the vicinity of Mackay Glacier

\begin{tabular}{|c|c|c|c|c|}
\hline \multirow{2}{*}{ Location } & \multirow{2}{*}{ Dating Context } & \multicolumn{2}{|c|}{ Timing } & \multirow{2}{*}{ Study } \\
\hline & & Corr. ${ }^{14} \mathrm{C}$ yr $(2 \sigma)$ & Cal. yr $(2 \sigma)$ & \\
\hline North of Ross Island & Retreat of grounded ice * & $8861 \pm 60$ & $10185-10005$ & Mckay et al. (2008) \\
\hline Explorers Cove & Presence of grounded ice & $8340 \pm 120$ & $9522-9268$ & Hall and Denton (2000) \\
\hline Scott Coast, McMurdo Sound (multiple sites) & Final unloading of grounded ice & $6600 \dagger$ & $7520-7465$ & Hall et al. (2004) \\
\hline Cape Bird & Retreat of grounded ice & $6530 \pm 60$ & $7545-7335$ & Licht et al. (1996) \\
\hline Marble Point & Ice shelf free & $5480 \pm 56$ & $6420-6178$ & Hall and Denton $(1999,2000)$ \\
\hline Explorers Cove & Retreat of grounded ice & $5370 \pm 200$ & $6401-5916$ & Hall and Denton (2000) \\
\hline Granite Harbour & Ice shelf free & $5480 \pm 65$ & $6378-6242$ & Domack et al. (1999) \\
\hline Gneiss Point & Ice shelf free & $5220 \pm 55$ & $6160-5906$ & Hall and Denton $(1999,2000)$ \\
\hline Cape Bernacchi & Ice shelf free & $4300 \pm 50$ & $4981-4846$ & Hall and Denton $(1999,2000)$ \\
\hline
\end{tabular}

Corrected (corr.) ${ }^{14} \mathrm{C}$ ages are derived from the original study and a recent review of regional deglaciation (Anderson et al., 2014). Calibrated (cal.) age ranges use the Marine09 calibration curve.

* Core sample that dates open water conditions and, by implication, minimum age of grounded ice retreat.

$\dagger$ Age was derived from a relative sea level curve comprising multiple samples, and therefore no uncertainty is attached. For calibration, an uncertainty of 50 years was used.

GZWs in West Antarctica indicate that they typically form within $\sim 120$ years if the sediment flux is sufficiently high (Graham et al., 2010).

The surface-exposure chronology from Mackay Glacier indicates that rapid, uninterrupted thinning occurred over at least four centuries. This most likely happened after the glacier retreated from the GZWs of the outer trough to the inner parts of Mackay Sea Valley, during a time when the large buttressing effect provided to Mackay Glacier by grounded ice, and possibly an ice-shelf, in the Ross Sea was removed. Offshore chronologies indicate that these conditions were met by $\sim 7.5 \mathrm{ka}$ and $\sim 6.3 \mathrm{ka}$ respectively, which is consistent with the new onshore chronology where rapid thinning is recorded between either $\sim 6.8$ and $6.0 \mathrm{ka}$ or $\sim 7.8$ and $7.0 \mathrm{ka}$, depending on the ${ }^{10} \mathrm{Be}$ production rate used.

\subsubsection{Mechanisms of rapid thinning at Mackay Glacier}

Dynamic glacier thinning requires a reduction of resistive stresses at the bed, groundingline or near the terminus (Bindschadler and Choi, 2007; Dupont and Alley, 2005; Howat et al., 2007; Jenkins et al., 2010; Nick et al., 2009; Stearns et al., 2008). As outlined above, regional geological evidence indicates that the most likely processes that drove rapid thinning of Mackay Glacier were retreat over a reverse bed slope into Mackay Sea Valley and/or removal of a local ice-shelf. Each of these processes will now be discussed in turn.

Bathymetric profiles of Mackay Sea Valley and trough reveal an overdeepening, which could have resulted in Mackay Glacier retreating as a consequence of Marine Ice Sheet Instability (Joughin and Alley, 2011). Grounding-lines located on reverse bed slopes are susceptible to this instability; initial retreat into deeper water leads to thicker ice at 


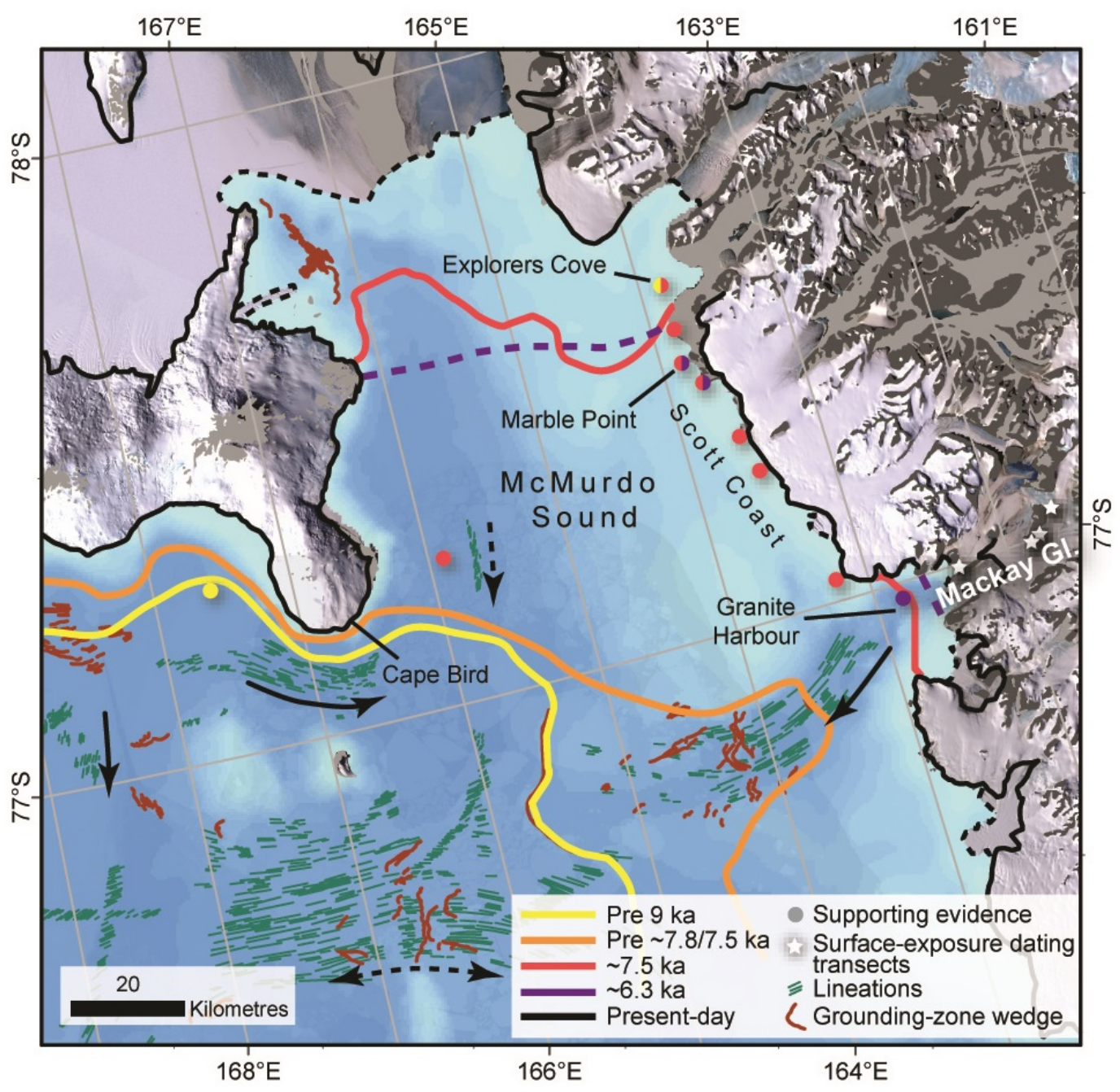

Figure 7.8 Deglaciation in the vicinity of Mackay Glacier. Reconstructed grounding-line (solid lines) and ice-shelf (dashed lines) retreat downstream of Mackay Glacier is supported by local chronological evidence (Table 7.3) and offshore geomorphology

(Greenwood et al., 2012). Arrows denote inferred flow of past grounded ice.

the grounding-line that is closer to floatation, leading to increased ice flux and glacier thinning, and resulting in further grounding-line retreat and drawdown of ice (Howat et al., 2007; Schoof, 2007; Vieli et al., 2001). The loss of buttressing from ungrounding at the terminus during periods of rapid grounding-line retreat produces a dynamic adjustment where thinning is propagated upstream (Howat et al., 2007; Nick et al., 2009; Vieli and Nick, 2011). The confined lower reaches of Mackay Glacier during the early to midHolocene were most likely susceptible to such upstream propagation; today, high surface velocities (Rignot et al., 2011) and likely abundant basal sliding (Pattyn, 2010) help to quickly redistribute ice mass (Howat et al., 2007). Abundant roche moutonées and striae on Cuff Cape near the current terminus indicate that these warm-based conditions also existed in the recent past (Möller, 1995).

Geomorphological evidence from the region suggests that Mackay Glacier may have also been influenced by the loss of an adjacent ice-shelf. Ice-shelf presence may have acted to 

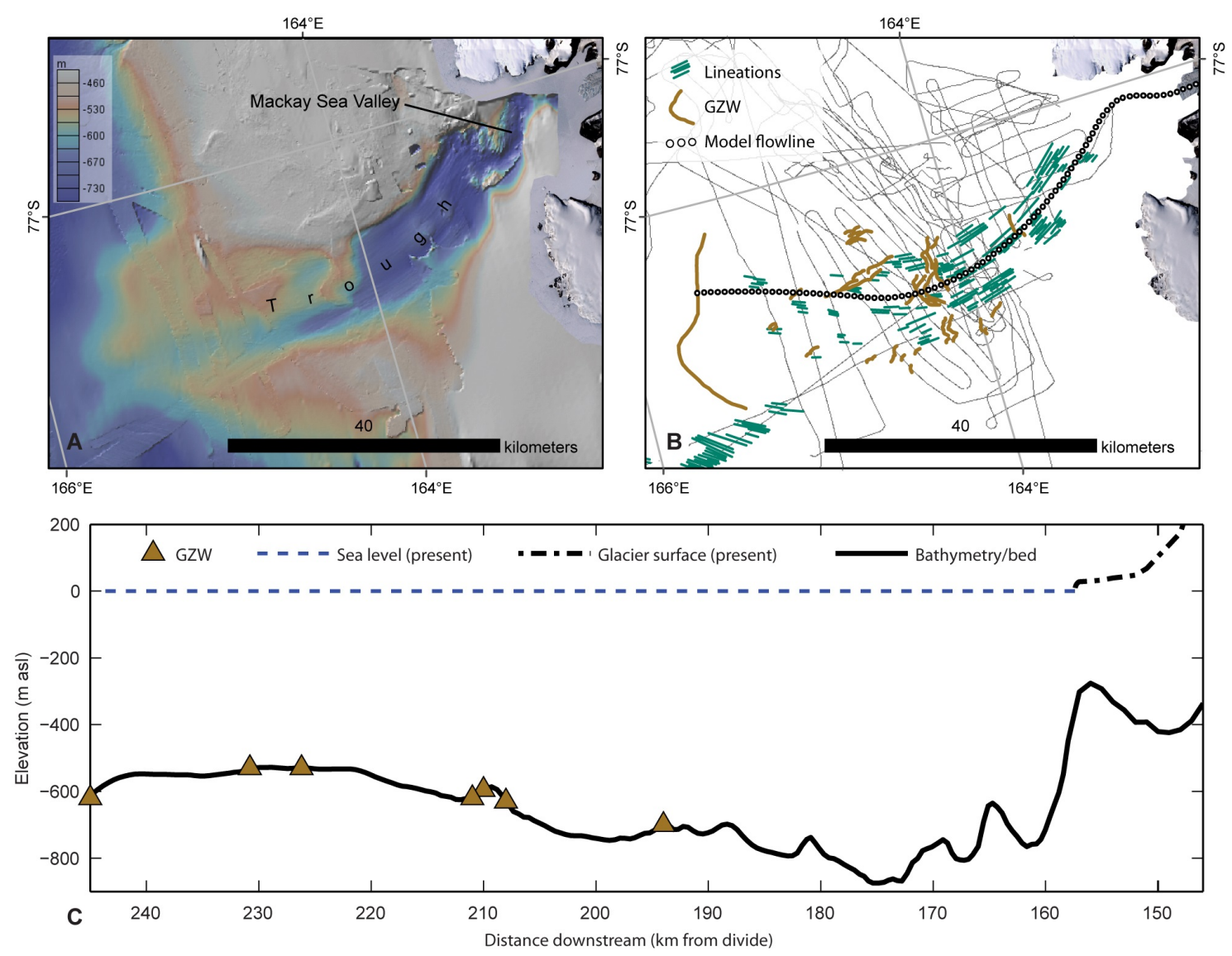

Figure 7.9 Staggered grounding-line retreat and reverse bed slope of Mackay Glacier offshore trough. GeoMapApp multi-resolution bathymetry (A) reveals lineations and grounding-zone wedges (GZW) mapped in B (Greenwood et al., 2012). High resolution multibeam tracks and the flowline (1 km horizontal resolution) used in model experiments are also shown. C) Longitudinal profile of Mackay Glacier trough from high resolution bathymetry data, with GZWs suggesting staggered retreat prior to grounding-line retreat into the overdeepening. Bed elevation beneath the tongue was obtained from geophysical surveys (Calkin, 1974), and the ice surface comes from airborne radar (ICECAP, D. Young pers. comm.).

slow or halt retreat at Mackay Glacier during its earliest stages, even if the grounding-line was positioned on a reverse bed slope (Payne et al., 2004; Rignot et al., 2004; Scambos et al., 2004; Schoof, 2007). Some GZWs are located on a reverse bed slope within the Mackay Glacier trough (Figure 7.9), possibly suggesting that a supporting ice-shelf was present at this time, although similar short-term grounding-line stability can be induced from changes in glacier width (Jamieson et al., 2012). The ultimate loss of this ice-shelf would have led to increased ice fluxes over the Mackay Glacier grounding-line (Dupont and Alley, 2006), possibly enabling a period of accelerated retreat and the rapid thinning recorded onshore.

Both of these scenarios involve rapid thinning of Mackay Glacier being driven by perturbations near its terminus, resulting in glacier retreat through an overdeepened trough. I next explore this retreat mechanism using numerical modelling. 


\subsection{Numerical flowline modelling of grounding-line retreat and upstream thinning}

\subsubsection{Set-up of flowline model}

In an attempt to understand whether progressive retreat of Mackay Glacier through its overdeepened trough resulted in the rapid thinning recorded, I simulated the glacier using a 1-dimensional finite difference flowline model. The model used here has been previously applied to Transantarctic Mountain outlet glaciers (Chapter 3; Golledge and Levy, 2011). Ice thickness change is determined using the mass conservation equation, as the balance between ice flux and net accumulation, which is then integrated through time. The shallow-ice approximation is used in combination with a longitudinal averaging scheme to calculate basal shear stress at 1-km horizontal resolution. As the past ice-shelf extent is not well constrained in time or space, I only implement the numerical scheme upstream of the grounding-line and do not simulate an ice-shelf.

Basal sliding in the model occurs where basal temperatures are close to melting point, while flow through creep is controlled by a temperature-dependent deformation rate with an enhancement coefficient. Here, a spatially variable basal sliding coefficient is used to account for differences in basal traction between the higher elevation Transantarctic Mountains portion, where Mackay Glacier is currently grounded on bedrock, and the downstream marine portion, where soft sediments occur. Enhanced sliding in the marine portion is suggested by the occurrence of elongate glacial lineations in the Mackay Glacier trough (Figure 7.9), indicative of former fast ice flow (Graham et al., 2009) from subglacial deformation over softer sediment (Hindmarsh, 1998).

Although ice-shelves are not directly incorporated into the model, negative mass balance is imposed for ice grounded below sea level downstream of the grounding-line. Sub-shelf melting is calculated using an ocean temperature-based melt scheme when the ice thickness at the grounding-line is less than floatation thickness (Holland et al., 2008; Golledge and Levy, 2011), controlled by a given seasonal ocean temperature cycle. Otherwise, mass loss at the grounding-line occurs from tidewater calving using a scalable calving rate coefficient as a factor of a calving constant (17) (Benn et al., 2007; Cuffey and Paterson, 2010; Golledge and Levy, 2011).

The model was initialised with bed topography and ice thickness obtained from multiple radar data profiles (Figure 7.10). Glacier surface elevations were obtained from airborne altimetry measurements (ICECAP), while the bed topography was interpolated from BEDMAP2 data (Fretwell et al., 2013) that was corrected along the flowline with 
radio-echoed ice thickness measurements (Calkin, 1974). The model domain extends over $244 \mathrm{~km}$ from the ice divide near Taylor Dome to the most distal offshore GZW (Figures 7.1B and 7.9). The flowline used for Mackay Glacier followed the sinuous yet laterally-constrained flow path through the Transantarctic Mountains, and was determined from present-day surface velocities, surface flow stripes, offshore lineations and trough bathymetry. In order to evaluate the thinning response resulting from migration of the grounding-line without effects from lateral variations (Jamieson et al., 2012), I applied a uniform width of $8 \mathrm{~km}$ along the length of the model domain, which approximates the glacier width in the lower reaches of the modern Mackay Glacier and the offshore trough.

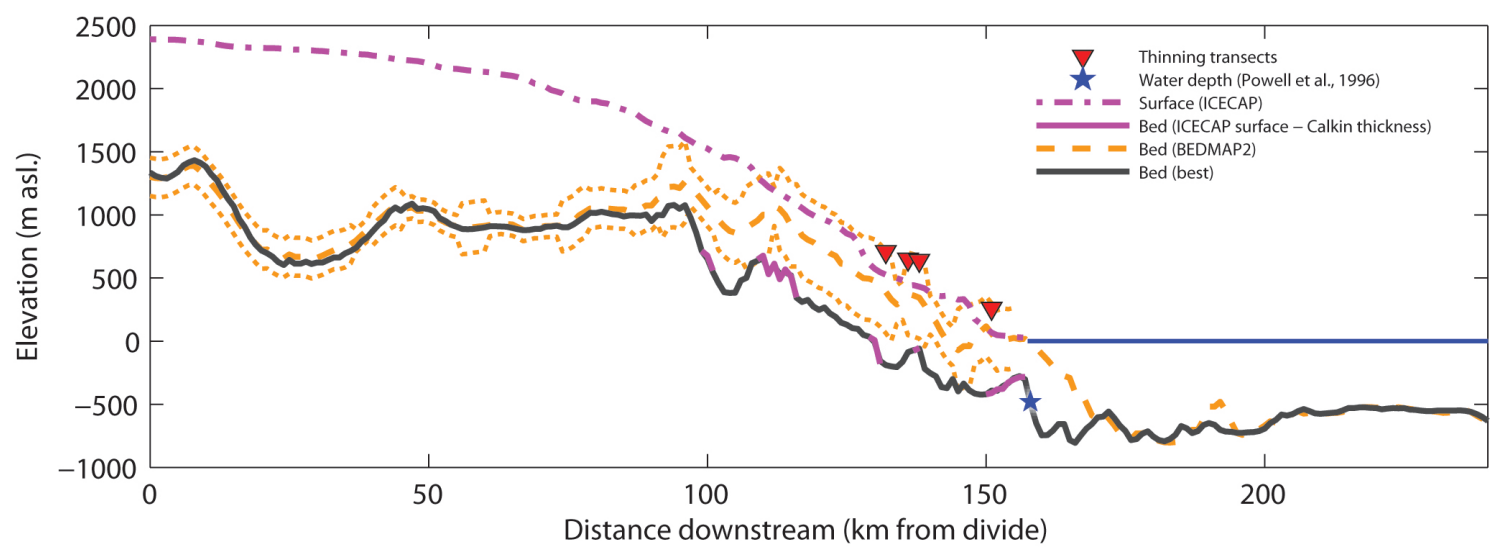

Figure 7.10 Ice surface and bed elevation estimations at Mackay Glacier. The ice surface is well-constrained by airborne radar (ICECAP, D. Young pers. comm.), however the bed topography is less certain. In the confined lower reaches, a lack of bed surveys contribute to the BEDMAP2 dataset (Fretwell et al., 2013), resulting in large uncertainty estimates and a mean bed elevation above sea level in the area of Mackay Glacier's floating tongue. Using radio-echo-derived ice thickness data (Calkin, 1974) and the measured ice surface (ICECAP), BEDMAP2 bed topography was corrected in the lower reaches. Beyond the present-day terminus, a measured water depth (Powell et al., 1996) and high-resolution bathymetry (IBCSO, Fig. 7.9c) constrained the bed. Modern sea level is shown as a blue line.

Model experiments used climate and glaciological parameterisations from field measurements and commonly used physical values (Table 7.4). Firstly, modern and advanced steady-state ice surface profiles were simulated by adjusting the precipitation at sea level, precipitation lapse rate, calving coefficient, ocean temperature, and basal sliding and creep enhancement factors. I simulated the modern ice surface at the sampling transects with a grounding-line located upstream of the large overdeepening. A more extensive 'advanced' glacier was simulated with the grounding-line beyond the end of the domain and the surface elevation $>100 \mathrm{~m}$ above the Mt Suess/Gondola Ridge transect, where an LGM ice surface is recorded prior to rapid thinning. This advanced state was simulated by decreasing both the calving coefficient and ocean temperature while maintaining the other tuned parameters of the present-day simulation. 
Table 7.4 Flowline model parameters

\begin{tabular}{lccc}
\hline \multirow{2}{*}{ Parameter } & \multicolumn{2}{c}{ Value } & \multirow{2}{*}{ Unit } \\
\hline Flow enhancement factor & 8 & 8 & - \\
Sliding rate factor (TAM) & $5 \times 10^{-8}$ & $5 \times 10^{-8}$ & $\mathrm{~Pa} \mathrm{~m}^{2} \mathrm{a}^{-1}$ \\
Sliding rate factor (Marine) & $2 \times 10^{-5}$ & $2 \times 10^{-5}$ & $\mathrm{~Pa} \mathrm{~m}^{2} \mathrm{a}^{-1}$ \\
Glen's flow law exponent & 3 & 3 & - \\
Sliding exponent & 2 & 2 & - \\
Geothermal heat flux & 58 & 58 & $\mathrm{~mW} \mathrm{~m}^{-2}$ \\
Horizontal domain resolution & 1000 & 1000 & $\mathrm{~m}^{-}$ \\
Valley width & 8000 & 8000 & $\mathrm{~m}$ \\
& & & \\
Precipitation at sea level * & 1.5 & 0.4 & $\mathrm{~m} \mathrm{a}^{-1}$ \\
Precipitation lapse rate $*$ & -0.01 & -0.01 & $\mathrm{~m} \mathrm{a}^{-1} \mathrm{~km}^{-1}$ \\
Mean annual temp. at sea level $\dagger$ & -17 & -17 & ${ }^{\circ} \mathrm{C}$ \\
Temperature lapse rate & -9 & -9 & $\mathrm{~K} \mathrm{~km}^{-1}$ \\
Annual temperature range $\dagger$ & 35 & 35 & ${ }^{\circ} \mathrm{C}$ \\
Ocean temperature (winter) & -1.8 & -1.8 & ${ }^{\circ} \mathrm{C}$ \\
Ocean temperature (summer) $*$ & -1.6 & -1.8 & ${ }^{\circ} \mathrm{C}$ \\
Calving rate coefficient $*$ & 1 & 0.3 & - \\
Relative sea level & 0 & 0 & $\mathrm{~m}^{-1}$ \\
Degree-day factor & 0.005 & 0.005 & $\mathrm{~mm} \mathrm{~K}^{-1} \mathrm{~d}^{-1}$ \\
\hline
\end{tabular}

*Tuning parameter. †Data from National Soil Survey Centre / Landcare Research. Reduced precipitation assumed for advanced profile (Steig et al., 2000).

Sliding rate factors are of the Transantarctic Mountains (TAM) and marine portions.

\subsubsection{Simulated thinning from grounding-line retreat}

In order to assess whether the change in surface elevation at the Mackay Glacier thinning transects could be explained simply by recession of the grounding-line, I forced the grounding-line from the outer trough, through the zone of GZWs and across the reverse bed slope of the inner trough. This was achieved using a range of individual summer ocean temperature and calving rate scenarios as the forcing. No retreat scenarios simulated ice thicker than floatation with a grounding-line positioned within the trough in the model domain, and therefore all negative changes in mass balance were achieved through basal melt at the grounding-line. In order to investigate the effects of pulses of sea level rise on the glacier, for example if the small rate of eustatic sea level rise was greater than that of isostatic rebound, I also simulated retreat from increased sea level scenarios. In all experiments, it was possible to simulate retreat from the outermost GZW to upstream of the overdeepened Mackay Sea Valley over 300-400 years (Figure 7.11). 


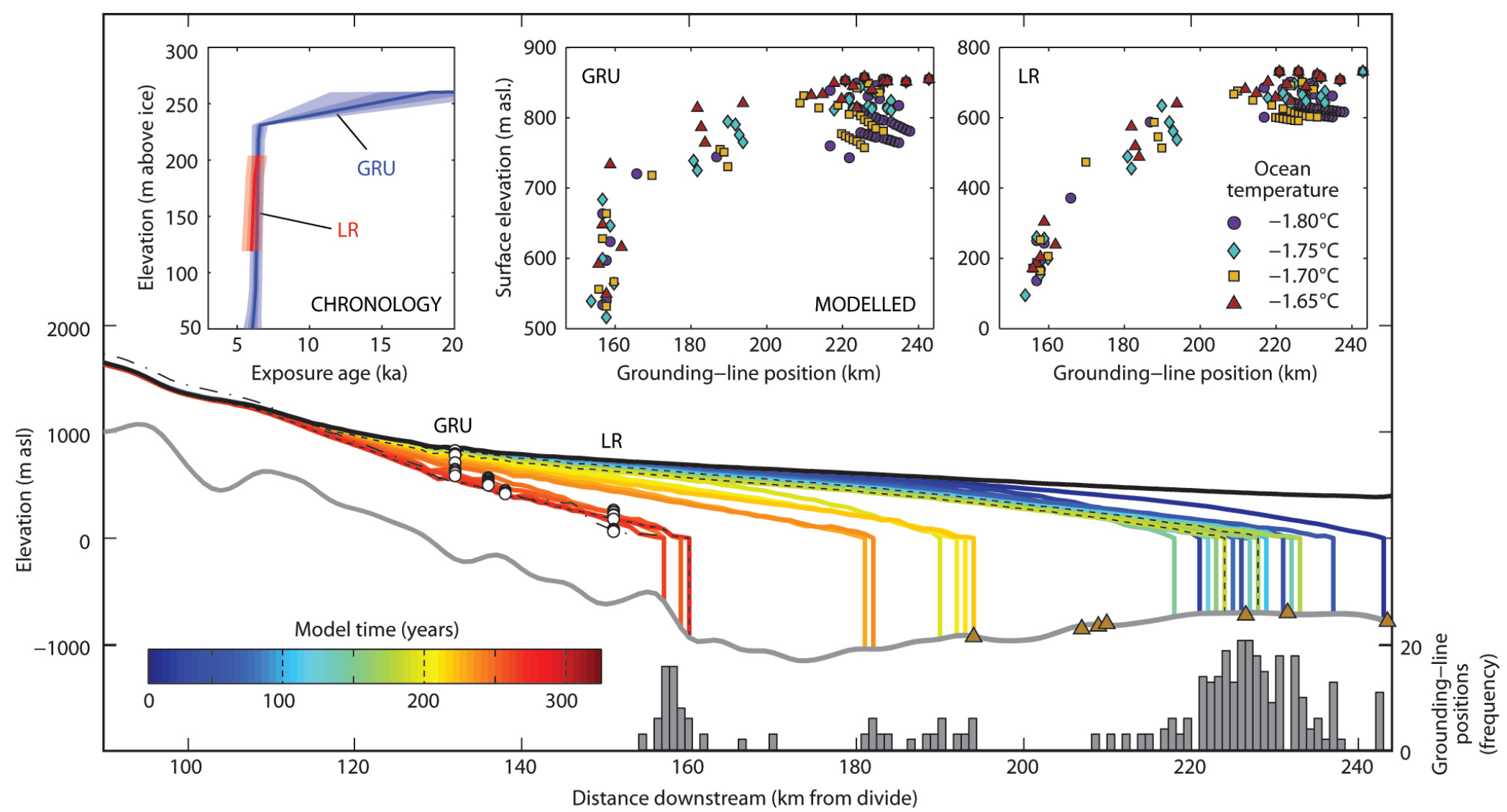

Figure 7.11 Modelled retreat of Mackay Glacier through the overdeepened trough with corresponding accelerated upstream thinning at the sample transects. An evolving surface profile is shown at 10-year intervals for one model run $\left(-1.75{ }^{\circ} \mathrm{C}\right.$ ocean temperature forcing scenario). A histogram displays the frequency of grounding-line positions for all modelled scenarios, with peaks broadly corresponding to most grounding-zone wedges (brown triangles). This modelling identifies that stable glacier positions occur in the outer trough, unstable fast retreat occurs on the reverse bed slope and then restored stability occurs in the vicinity of the present-day grounding line. Simulated surface thinning at the Mt Suess/Gondola Ridge (upper) (GRU) and

Low Ridge (LR) transects is shown in scatter plots for a range of ocean forcing scenarios. Some initial thinning occurs in all scenarios and at both transects when the grounding-line is located in the outer trough. Accelerated surface lowering occurs first at LR simultaneous with retreat over the reverse bed slope, while the most rapid thinning is simulated upstream at GRU, matching the surface lowering chronologies. Sample locations are denoted by white circles. 
In all retreat scenarios, the grounding-line fluctuates in the outer trough, retreats more rapidly over the reverse bed slope and then re-establishes stability on the normal slope of the bedrock ridge upstream of the overdeepening (Figure 7.11). A higher frequency of grounding-line positions was simulated in the outer trough, which in most cases match observed GZWs (Greenwood et al., 2012). In some cases (for example, at $\sim 210 \mathrm{~km}$ from the divide), temporary grounding-line stability that is inferred from the presence of GZWs is not simulated in any retreat scenarios. This could be because of reduced trough width and complex lateral drag in this location (Figure 7.9), a stability feedback provided by the positive relief of GZW deposition, and/or enhanced stability from the presence of an ice-shelf at that time, none of which were incorporated within the model. In the overdeepening, retreat into deeper water allows for thicker ice at the grounding-line that is closer to floatation, leading to greater ice fluxes and therefore further retreat (Figure 7.12).

While grounding-line retreat is generally faster for warmer ocean temperatures and higher relative sea level, the pattern of upstream thinning is similar in all forcing scenarios (Figures 7.11 and 7.13A); accelerated upstream thinning occurs as the grounding-line retreats

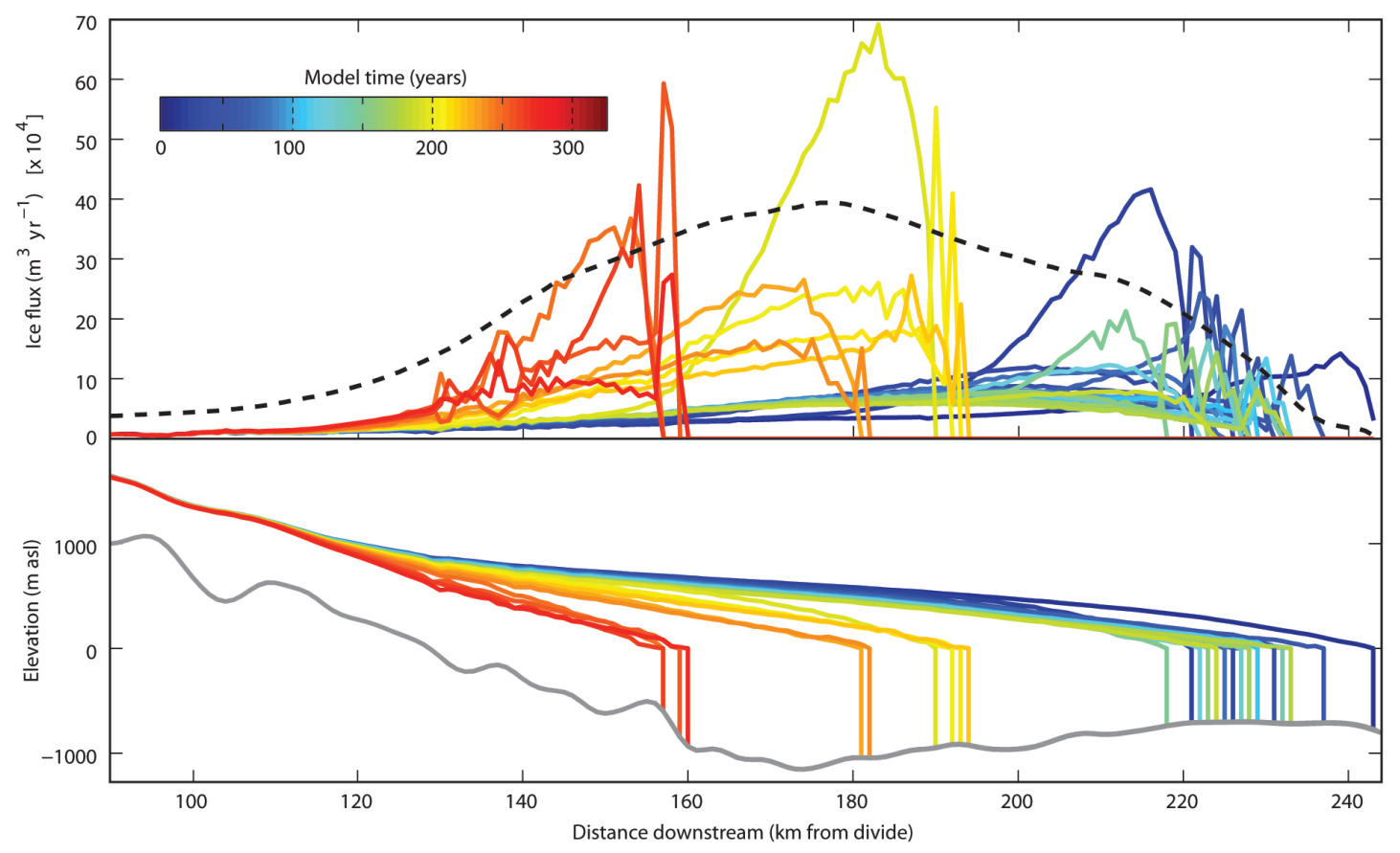

Figure 7.12 Modelled ice flux during retreat of Mackay Glacier through an overdeepened trough. An evolving ice flux (upper panel) corresponds to the surface profile (lower panel), shown at 10-year intervals as in Figure 7.11. As the grounding-line retreats over the reverse bed slope, thicker ice allows for greater ice fluxes and therefore potential for mass loss. The largest flux of ice occurs in the deepest part of the trough, where the glacier is thickest and a steep ice surface slope facilitates higher velocities. Ice

flux remains relatively large until the glacier stabilizes on the normal-sloping bed, temporarily maintained by high surface velocities. The dashed black line denotes the longitudinally-averaged $(25 \mathrm{~km})$ ice flux $\left(5 \times 10^{4}\right)$ over this retreat period. 
over the reverse bed slope. The increased rate of surface lowering is initially experienced at Low Ridge. Subsequently, a more rapid thinning response occurs at Mt Suess/Gondola Ridge (upper). This spatial pattern of ice-dynamic thinning fits the thinning gradients of the respective transect chronologies and the idea of propagated upstream thinning following perturbations at the grounding-line (Jamieson et al., 2012; Nick et al., 2009; Payne et al., 2004).
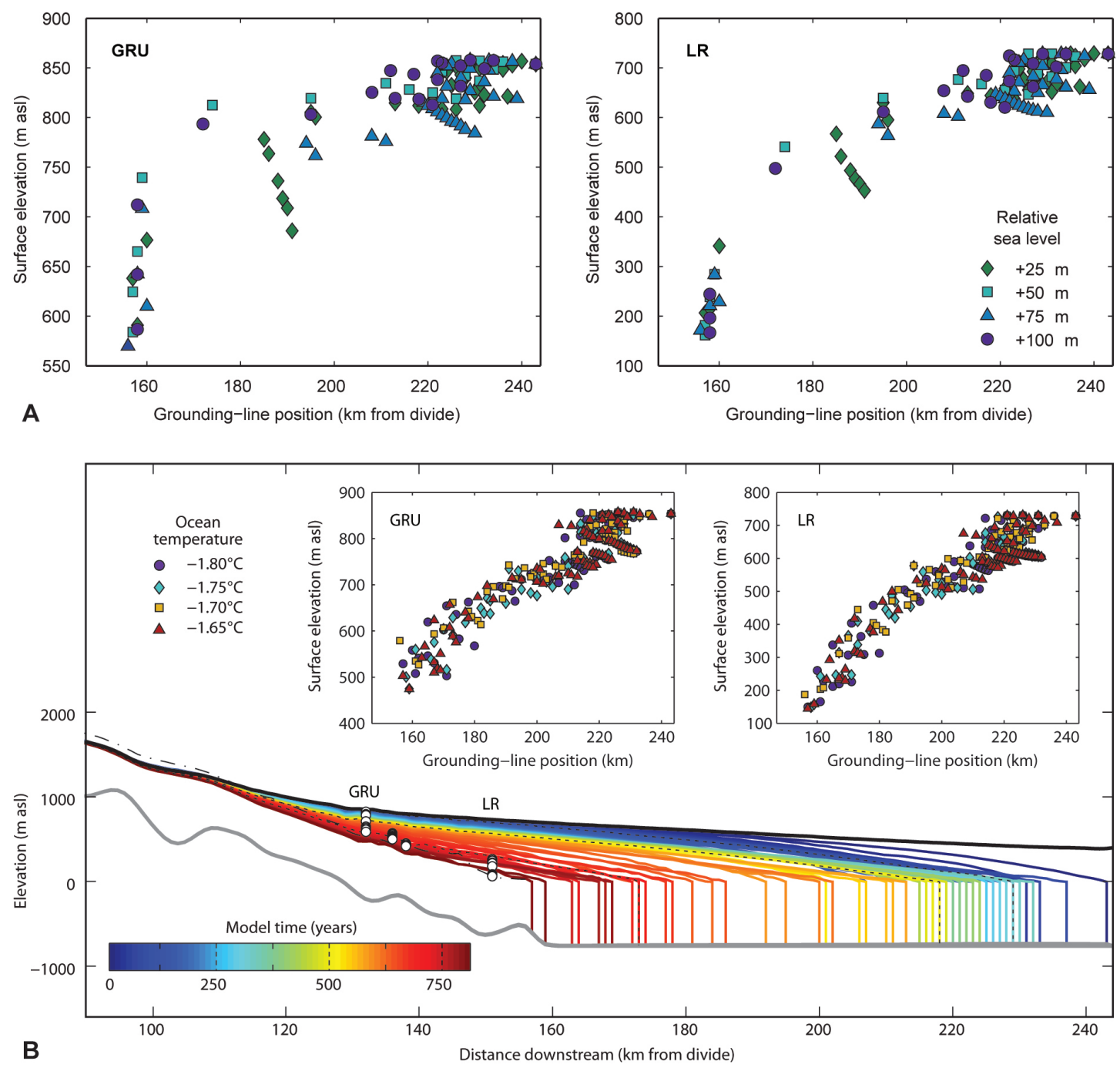

Figure 7.13 Model experiments of grounding-line retreat and surface thinning at the sample transects. A) Retreat driven by enhanced relative sea level. The pattern of surface lowering at Mt Suess/Gondola Ridge (upper) (GRU) and Low Ridge (LR), of gradual and then accelerated thinning, is largely consistent with that of enhanced ocean temperature (Fig. 7.11). B) Simulated retreat over an imposed flat bed, with an evolving surface profile as in Figure 7.11, shown for a $-1.65^{\circ} \mathrm{C}$ ocean temperature forcing scenario. Retreat lasts for $\sim 800$ years and surface lowering responds linearly. This indicates that the rapid thinning recorded in the Mackay Glacier transects can be explained by a primary topographic control, largely irrespective of any single environmental forcing. 
Non-linear dynamic thinning in the model may have occurred due to a process other than the reverse bed slope. Therefore, an idealised experiment was carried out in which Mackay Sea Valley was replaced by a flat bed (Figure 7.13B). Under this modified bed geometry, modelled retreat occurs over a longer period of $\sim 800$ years without substantial jumps in the grounding-line position. Retreat accelerates slightly towards the modern configuration, which is probably the result of a steepening surface slope and minor increases in ice flux as the grounding-line approaches the high-relief Transantarctic Mountains portion. However, in all forcing scenarios, surface lowering is linear and, therefore, fails to replicate the rapid thinning recorded in the Mt Suess/Gondola Ridge (upper) and Low Ridge transects.

Together, the modelling experiments and regional geological constraints suggest that grounding-line retreat into an overdeepened trough was the most likely mechanism that led to the substantial thinning recorded at the nunatak sites. Reduced buttressing from ice-shelf disintegration (Pattyn et al., 2006; Schoof, 2007) is not required to explain rapid retreat and dynamic thinning but it could have helped facilitate this retreat.

\subsection{Wider empirical records of Antarctic deglaciation}

Glacial troughs excavated to more than $1 \mathrm{~km}$ below sea level and their associated reverse bed slopes are common features on the inner continental-shelf (Fretwell et al., 2013; Livingstone et al., 2012; Mackintosh et al., 2011). Ice-dynamic thinning and rapid mass loss may therefore have occurred elsewhere when grounding-lines retreated through these troughs. I next assess whether the thinning history observed at Mackay Glacier is unique or part of a wider pattern of Antarctic ice sheet retreat, and discuss whether such changes can be attributed to climatic or topographic factors.

\subsubsection{Evidence of circum-Antarctic deglaciation}

Recent reviews of Antarctic geological records that document deglaciation from the LGM (Anderson et al., 2014; O'Cofaigh et al., 2014; Hillenbrand et al., 2014; Larter et al., 2014; Mackintosh et al., 2014) enable a new assessment of circum-Antarctic ice sheet response during the last deglaciation. All evidence of grounding-line retreat that was considered to have reliable age constraints was collated (Table 7.5), noting whether they record grounding-line retreat on the outer, mid or inner continental-shelf for several submarine troughs (Livingstone et al., 2012). Age corrections were made based on the original and/or reviewed sources, and all radiocarbon dates provide minimum age constraints. 
The onset of grounding-line retreat and the timing of retreat to positions on the mid and inner shelf were highly variable for both the West Antarctic Ice Sheet (WAIS) and EAIS (Figure 7.14). Such variation between troughs implies a likely dominant control of local topography on rates of grounding-line retreat (Livingstone et al., 2012). Furthermore, all records show that grounding-lines had retreated to the inner-shelf before $\sim 8 \mathrm{ka}$, and largely before $\sim 10 \mathrm{ka}$.

To investigate the associated upstream surface lowering, the circum-Antarctic evidence of ice sheet thinning from "dipstick" records was compared (Table 7.6). As discussed above, the smaller $\mathrm{NZ}{ }^{10} \mathrm{Be}$ production rate (Putnam et al., 2010) produces artificially low precision Antarctic exposure ages, but also upper estimates for the onset of ice sheet surface lowering (for example, Figure 7.5). Therefore, by recalculating the circum-Antarctic "dipstick" records using the NZ production rate, I can assess the maximum timing of thinning relative to the minimum ages of grounding-line retreat on the continental-shelf. Stepwise linear regression was then applied to each "dipstick" transect to evaluate thinning trends following LGM surface highstands.

Initial gradual thinning is recorded in the WAIS, EAIS and Antarctic Peninsula Ice Sheet (APIS) from 30-15 ka (Bentley et al., 2006, 2010; Johnson et al., 2008, 2012; White et al., 2011b) (Figure 7.14). Prior to $\sim 15 \mathrm{ka},<200 \mathrm{~m}$ of upstream surface lowering occurred at a time of circum-Antarctic grounding-line retreat from the outer-shelf. Sections of EAIS experienced faster thinning from $\sim 15 \mathrm{ka}$, possibly as cross-shelf troughs deglaciated (Mackintosh et al., 2011). Ice-rafted debris events indicate an episode of mass loss from Antarctica at the time of Meltwater Pulse 1A (Weber et al., 2014), which was probably sourced from the Antarctic Peninsula and outer Weddell Sea (Golledge et al., 2014b). Critically, in the context of this new data at Mackay Glacier, a period of widespread rapid ice sheet thinning occurred in the Holocene, concentrated within a $\sim 1500$-year period ( $~ 8.0-6.5 \mathrm{ka}, \mathrm{NZ}$ production rate). This dramatic surface lowering is recorded in WAIS, APIS and EAIS (Balco and Schaefer, 2013; Bentley et al., 2010; Fogwill et al., 2012; Johnson et al., 2014) (including this study), after grounding-lines had retreated to the inner continental-shelf. Widespread evidence of ice sheet surface lowering suggests a common circum-Antarctic control. Grounding-lines were located on the inner-shelf during the Holocene and any variance in the timing of Holocene ice sheet thinning probably reflects local differences in basin topography and specifically, basin-to basin differences in when grounding-lines reached their respective overdeepenings. 
Table 7.5 Circum-Antarctic chronologies of West and East Antarctic grounding-line retreat

\begin{tabular}{|c|c|c|c|c|c|c|c|c|c|c|}
\hline Ice sheet & Sector & Site & Site ID & Location & Core & Latitude & Longitude & $\begin{array}{c}{ }^{14} \text { C age } \\
\text { (corr., cal. yrs) }\end{array}$ & $\begin{array}{l}{ }^{14} \text { C error } \\
(\text { cal., } 2 \sigma)\end{array}$ & Published source \\
\hline WAIS & Amundsen-Bellinghausen & Belgica Trough & $\mathrm{BeT}$ & Outer-shelf & GC372 & -70.605 & -86.253 & 30758 & 2262 & Hillenbrand et al. (2010) \\
\hline WAIS & Amundsen-Bellinghausen & Belgica Trough & BeT & Mid-shelf & GC368 & -71.578 & -82.860 & 23527 & 1984 & Hillenbrand et al. (2010) \\
\hline WAIS & Amundsen-Bellinghausen & Belgica Trough & $\mathrm{BeT}$ & Inner-shelf & GC366 & -72.845 & -82.615 & 14346 & 847 & Hillenbrand et al. (2010) \\
\hline WAIS & Amundsen-Bellinghausen & Pine Island Bay & PIB & Outer-shelf & PC07 & -71.250 & -109.000 & 16423 & 448 & Kirshner et al. (2012) \\
\hline WAIS & Amundsen-Bellinghausen & Pine Island Bay & PIB & Mid-shelf & ANT-XXVI/3 & -72.784 & -105.105 & 17805 & 578 & Klages et al. (2013) \\
\hline WAIS & Amundsen-Bellinghausen & Pine Island Bay & PIB & Inner-shelf & PS75/214-1 & -74.533 & -102.621 & 11157 & 248 & Hillenbrand et al. (2012) \\
\hline WAIS & Amundsen-Bellinghausen & Pine Island Bay & PIB & Inner-shelf & PC41 & -73.917 & -106.648 & 10086 & 947 & Lowe and Anderson (2002) \\
\hline WAIS & Amundsen-Bellinghausen & Dotzon-Getz Trough & DGT & Outer-shelf & VC436 & -71.814 & -117.434 & 22679 & 545 & Smith et al. (2011) \\
\hline WAIS & Amundsen-Bellinghausen & Dotzon-Getz Trough & DGT & Mid-shelf & VC428 & -73.143 & -115.704 & 13779 & 283 & Smith et al. (2011) \\
\hline WAIS & Amundsen-Bellinghausen & Dotzon-Getz Trough & DGT & Inner-shelf & VC415 & -73.896 & -115.931 & 10175 & 235 & Smith et al. (2011) \\
\hline WAIS & Amundsen-Bellinghausen & Dotzon-Getz Trough & DGT & Inner-shelf & PS75/129-1 & -74.509 & -134.121 & 12825 & 236 & Hillenbrand et al. (2012) \\
\hline WAIS & Amundsen-Bellinghausen & Wrigley Gulf Trough & WGT & Inner-shelf & PC26 & -74.256 & -128.374 & 15610 & 651 & Anderson et al. (2002) \\
\hline WAIS & Amundsen-Bellinghausen & Hobbs Coast trough & HCT & Inner-shelf & PS75/130-1 & -74.445 & -134.153 & 20875 & 814 & Klages et al. (2014) \\
\hline WAIS & Ross Sea & Central Ross Sea & CRS & Mid-shelf & PC2/PC7 & -76.402 & -177.297 & 30800 & - & Bart and Cone (2012) \\
\hline EAIS & Ross Sea & JOIDES Trough & JoT & Mid-shelf & KC39 & -74.473 & 173.512 & 13010 & 254 & Cunningham et al. (1999); Domack et al. (1999) \\
\hline EAIS & Ross Sea & Drygalski Trough & DrT & Mid-shelf & KC37 & -74.498 & 167.743 & 12930 & 239 & Cunningham et al. (1999); Domack et al. (1999) \\
\hline EAIS & Ross Sea & Drygalski Trough & DrT & Inner-shelf & KC31 & -75.700 & 165.417 & 11270 & 339 & Cunningham et al. (1999); Domack et al. (1999) \\
\hline EAIS & George V - Dronning-Maud Land & George V Land & GVL & Mid-shelf & 27PC13 & -66.520 & 143.383 & 11277 & 273 & Harris et al. (2001) \\
\hline EAIS & George V - Dronning-Maud Land & Rauer & Rau & Inner-shelf & Co1010 & -68.802 & 77.889 & 13058 & 323 & Berg et al. (2010) \\
\hline EAIS & George V - Dronning-Maud Land & Prydz Bay & $\operatorname{PrB}$ & Mid-shelf & KROCK-GC24 & -68.094 & 73.189 & 13245 & 346 & Domack et al. (1998) \\
\hline EAIS & George V - Dronning-Maud Land & Prydz Bay & $\operatorname{PrB}$ & Inner-shelf & $119-740 \mathrm{~A}-3 \mathrm{R}-2$ & -68.687 & 76.721 & 11271 & 377 & Domack et al. (1991) \\
\hline EAIS & George V - Dronning-Maud Land & Iceberg Alley & IcA & Outer-shelf & JPC43B & -66.932 & 63.123 & 11659 & 541 & Leventer et al. (2006) \\
\hline EAIS & George V - Dronning-Maud Land & Nielsen Basin & $\mathrm{NiB}$ & Mid-shelf & JPC40 & -67.176 & 65.737 & 14179 & 521 & Leventer et al. (2006) \\
\hline EAIS & George V - Dronning-Maud Land & Lazarev Sea & $\mathrm{LaS}$ & Mid-shelf & PS2028 & -70.013 & 11.755 & 11290 & 501 & Gingele et al. (1997) \\
\hline EAIS & Weddell Sea & Quar Ice Shelf & QIS & Outer-shelf & $3-17-1$ & -71.000 & -11.750 & 27290 & 612 & Anderson and Andrews (1999); Stolldorf et al. (2012) \\
\hline EAIS & Weddell Sea & Riiser-Larsen Ice Shelf & RLS & Outer-shelf & A85-10 & -72.348 & -16.495 & 20991 & 762 & Kristoffersen et al. (2000b) \\
\hline EAIS & Weddell Sea & Riiser-Larsen Ice Shelf & RLS & Mid-shelf & KK9601 & -72.516 & -16.534 & 11723 & 442 & Kristoffersen et al. (2000a) \\
\hline EAIS & Weddell Sea & Filchner Trough & FiT & Outer-shelf & $2-19-1$ & -74.350 & -38.250 & 18173 & 361 & Anderson and Andrews (1999); Stolldorf et al. (2012) \\
\hline EAIS & Weddell Sea & Filchner Trough & FiT & Inner-shelf & G7 & -77.333 & -36.550 & 8676 & 297 & Stolldorf et al. (2012) \\
\hline
\end{tabular}

WAIS and EAIS are West and East Antarctic Ice Sheets, respectively. Each outlet system is represented by a Site ID, included in Figure 7.14. 
Table 7.6 Circum-Antarctic "dipstick" thinning transects

\begin{tabular}{|c|c|c|c|c|c|c|c|c|c|c|c|c|}
\hline Region & Site & Sample ID & Latitude & Longitude & $\begin{array}{l}\text { Altitude } \\
\text { (m asl) }\end{array}$ & $\begin{array}{l}\text { Elevation } \\
\text { above } \\
\text { modern } \\
\text { ice }(\mathbf{m})\end{array}$ & $\begin{array}{c}\text { Cosmogenic } \\
\text { nuclide }\end{array}$ & $\begin{array}{c}\text { Published } \\
\text { age (yrs) }\end{array}$ & $\begin{array}{l}\text { Published } \\
\text { error (yrs) }\end{array}$ & Published source & $\begin{array}{l}\text { Recalc. age } \\
\text { (yrs) (NZ) }\end{array}$ & $\begin{array}{l}\text { Recalc. error } \\
\text { (yrs) (NZ) }\end{array}$ \\
\hline Marie Byrd Land (WAIS) & Mt Rea (The Billboard) & 99-MBL-056-BBD & -77.072 & -145.688 & 715 & 620 & ${ }^{10} \mathrm{Be}$ & 10400 & 680 & Stone et al. (2003) & 12267 & 422 \\
\hline Marie Byrd Land (WAIS) & Mt Rea (The Billboard) & 99-MBL-061-REA & -77.068 & -145.574 & 489 & 394 & ${ }^{10} \mathrm{Be}$ & 3120 & 270 & Stone et al. (2003) & 3649 & 244 \\
\hline Marie Byrd Land (WAIS) & Mt Rea (The Billboard) & 99-MBL-062-REA & -77.068 & -145.574 & 489 & 394 & ${ }^{10} \mathrm{Be}$ & 3390 & 230 & Stone et al. (2003) & 3988 & 156 \\
\hline Marie Byrd Land (WAIS) & Mt Rea (The Billboard) & 01-MBL-151-REA & -77.068 & -145.565 & 433 & 338 & ${ }^{10} \mathrm{Be}$ & 3360 & 240 & Stone et al. (2003) & 3931 & 174 \\
\hline Marie Byrd Land (WAIS) & Mt Rea (The Billboard) & 01-MBL-153-REA & -77.068 & -145.565 & 404 & 309 & ${ }^{10} \mathrm{Be}$ & 2750 & 200 & Stone et al. (2003) & 3225 & 150 \\
\hline Marie Byrd Land (WAIS) & Mt Rea (The Billboard) & 99-MBL-066-REA & -77.068 & -145.548 & 287 & 192 & ${ }^{10} \mathrm{Be}$ & 2740 & 190 & Stone et al. (2003) & 3203 & 125 \\
\hline Marie Byrd Land (WAIS) & Mt Rea (The Billboard) & 01-MBL-161A-REA & -77.061 & -145.57 & 172 & 77 & ${ }^{10} \mathrm{Be}$ & 2600 & 220 & Stone et al. (2003) & 3047 & 208 \\
\hline Marie Byrd Land (WAIS) & Mt Rea (The Billboard) & 01-MBL-163-REA & -77.060 & -145.569 & 162 & 67 & ${ }^{10} \mathrm{Be}$ & 2380 & 210 & Stone et al. (2003) & 2770 & 195 \\
\hline Marie Byrd Land (WAIS) & Mt Rea / Mt Dolber col & 99-MBL-080-GAP & -77.084 & -145.558 & 352 & 257 & ${ }^{10} \mathrm{Be}$ & 2370 & 230 & Stone et al. (2003) & 2775 & 228 \\
\hline Marie Byrd Land (WAIS) & Mt Rea / Mt Dolber col & 99-MBL-081-GAP & -77.084 & -145.558 & 350 & 255 & ${ }^{10} \mathrm{Be}$ & 2480 & 200 & Stone et al. (2003) & 2908 & 177 \\
\hline Marie Byrd Land (WAIS) & Mt Rea / Mt Dolber col & 99-MBL-083/3-GAP & -77.082 & -145.546 & 245 & 150 & ${ }^{10} \mathrm{Be}$ & 1940 & 160 & Stone et al. (2003) & 2273 & 132 \\
\hline Marie Byrd Land (WAIS) & Mt Rea / Mt Dolber col & 99-MBL-084-GAP & -77.082 & -145.545 & 237 & 142 & ${ }^{10} \mathrm{Be}$ & 590 & 70 & Stone et al. (2003) & 687 & 79 \\
\hline Marie Byrd Land (WAIS) & Mt Rea / Mt Dolber col & 01-MBL-157/3-GAP & -77.081 & -145.544 & 226 & 131 & ${ }^{10} \mathrm{Be}$ & 300 & 90 & Stone et al. (2003) & 353 & 113 \\
\hline Pine Island (WAIS) & Maish Nunatak & JF-01 & -74.589 & -99.451 & 286 & 106 & ${ }^{10} \mathrm{Be}$ & 8111 & 287 & Johnson et al. (2014) & 8116 & 287 \\
\hline Pine Island (WAIS) & Maish Nunatak & $\mathrm{JF}-02$ & -74.589 & -99.449 & 268 & 88 & ${ }^{10} \mathrm{Be}$ & 7917 & 282 & Johnson et al. (2014) & 7927 & 282 \\
\hline Pine Island (WAIS) & Maish Nunatak & JF-04 & -74.591 & -99.441 & 255 & 75 & ${ }^{10} \mathrm{Be}$ & 7898 & 285 & Johnson et al. (2014) & 7905 & 285 \\
\hline Pine Island (WAIS) & Maish Nunatak & JF-06 & -74.597 & -99.456 & 200 & 20 & ${ }^{10} \mathrm{Be}$ & 7675 & 285 & Johnson et al. (2014) & 7678 & 285 \\
\hline Pine Island (WAIS) & Maish Nunatak & JF-07 & -74.597 & -99.451 & 187 & 7 & ${ }^{10} \mathrm{Be}$ & 8139 & 286 & Johnson et al. (2014) & 8140 & 286 \\
\hline Pine Island (WAIS) & Mt Moses & MTM-01 & -74.551 & -99.189 & 366 & 142 & ${ }^{10} \mathrm{Be}$ & 7898 & 278 & Johnson et al. (2014) & 7905 & 278 \\
\hline Pine Island (WAIS) & Mt Moses & MTM-02 & -74.551 & -99.193 & 351 & 127 & ${ }^{10} \mathrm{Be}$ & 7918 & 285 & Johnson et al. (2014) & 7923 & 285 \\
\hline Pine Island (WAIS) & Mt Moses & MTM-03 & -74.553 & -99.192 & 310 & 86 & ${ }^{10} \mathrm{Be}$ & 7872 & 268 & Johnson et al. (2014) & 7879 & 268 \\
\hline Pine Island (WAIS) & Mt Moses & MTM-04 & -74.553 & -99.199 & 247 & 23 & ${ }^{10} \mathrm{Be}$ & 6306 & 223 & Johnson et al. (2014) & 6305 & 223 \\
\hline Pine Island (WAIS) & Mt Moses & MTM-05 & -74.554 & -99.197 & 244 & 20 & ${ }^{10} \mathrm{Be}$ & 6977 & 253 & Johnson et al. (2014) & 6980 & 254 \\
\hline Pine Island (WAIS) & Mt Moses & MTM-06 & -74.553 & -99.202 & 224 & 0 & ${ }^{10} \mathrm{Be}$ & 5963 & 210 & Johnson et al. (2014) & 5965 & 210 \\
\hline Pine Island (WAIS) & Turtle Rock & TR1 & -75.369 & -111.299 & 700 & 334 & ${ }^{10} \mathrm{Be}$ & 14453 & 1512 & Johnson et al. (2008) & 18947 & 1099 \\
\hline Pine Island (WAIS) & Turtle Rock & TR2 & -75.371 & -111.291 & 631 & 265 & ${ }^{10} \mathrm{Be}$ & 11700 & 1421 & Johnson et al. (2008) & 15308 & 1300 \\
\hline Ellsworth Mountains (WAIS) & Marble Hills (Heritage Range) & MAR-09-CJF & -80.263 & -82.099 & 1305 & 485 & ${ }^{10} \mathrm{Be}$ & 15100 & 1300 & Bentley et al. (2010); Fogwill et al. (2012) & 19970 & 456 \\
\hline Ellsworth Mountains (WAIS) & Marble Hills (Heritage Range) & MAR-11-CJF & -80.235 & -82.183 & 1280 & 460 & ${ }^{10} \mathrm{Be}$ & 15400 & 1400 & Bentley et al. (2010); Fogwill et al. (2012) & 20383 & 801 \\
\hline Ellsworth Mountains (WAIS) & Marble Hills (Heritage Range) & MAR-05-MJB & $\begin{array}{l}-80.263 \\
-80.262\end{array}$ & -82.169 & 1192 & 372 & ${ }^{10} \mathrm{Be}$ & 6800 & 600 & Bentley et al. (2010); Fogwill et al. (2012) & 8915 & 297 \\
\hline Ellsworth Mountains (WAIS) & Marble Hills (Heritage Range) & MAR-06-MJB & -80.262 & -82.170 & 1166 & 346 & ${ }^{10} \mathrm{Be}$ & 7400 & 700 & Bentley et al. (2010); Fogwill et al. (2012) & 9784 & 490 \\
\hline Ellsworth Mountains (WAIS) & Marble Hills (Heritage Range) & MAR-08-MJB & -80.261 & -82.086 & 1002 & 182 & ${ }^{10} \mathrm{Be}$ & 6200 & 500 & Bentley et al. (2010); Fogwill et al. (2012) & 8230 & 195 \\
\hline Ellsworth Mountains (WAIS) & Marble Hills (Heritage Range) & CF-228-08 & -80.257 & -82.133 & 986 & 166 & ${ }^{10} \mathrm{Be}$ & 5400 & 500 & Bentley et al. (2010); Fogwill et al. (2012) & 7120 & 217 \\
\hline Ellsworth Mountains (WAIS) & Marble Hills (Heritage Range) & CF-229-08 & -80.257 & -82.133 & 986 & 166 & ${ }^{10} \mathrm{Be}$ & 4600 & 400 & Bentley et al. (2010); Fogwill et al. (2012) & 6114 & 197 \\
\hline Ellsworth Mountains (WAIS) & Marble Hills (Heritage Range) & MAR-11-MJB & -80.237 & -82.170 & 810 & 0 & ${ }^{10} \mathrm{Be}$ & 2100 & 200 & Bentley et al. (2010); Fogwill et al. (2012) & 2797 & 89 \\
\hline Ellsworth Mountains (WAIS) & Marble Hills (Heritage Range) & MAR-12-MJB & -80.263 & -82.138 & 807 & 0 & ${ }^{10} \mathrm{Be}$ & 2800 & 300 & Bentley et al. (2010); Fogwill et al. (2012) & 3652 & 235 \\
\hline Ellsworth Mountains (WAIS) & Patriot Hills West (Heritage Range) & PAT-01-MJB & -80.330 & -81.365 & 1092 & 192 & ${ }^{10} \mathrm{Be}$ & 8200 & 800 & Bentley et al. (2010); Fogwill et al. (2012) & 10751 & 383 \\
\hline Ellsworth Mountains (WAIS) & Patriot Hills West (Heritage Range) & PAT-03-MJB & -80.331 & -81.387 & 1009 & 109 & ${ }^{10} \mathrm{Be}$ & 5700 & 1800 & Bentley et al. (2010); Fogwill et al. (2012) & 7455 & 279 \\
\hline Antarctic Peninsula (NE) & Sjögren/Boydell fjord (South) & 10-LAR-016-SJO & -64.268 & -59.083 & 358 & 108 & ${ }^{10} \mathrm{Be}$ & 7370 & 250 & Balco and Schaefer (2013) & 8213 & 253 \\
\hline Antarctic Peninsula (NE) & Sjögren/Boydell fjord (South) & 10-LAR-013-SJO & -64.266 & -59.081 & 343 & 93 & ${ }^{10} \mathrm{Be}$ & 6900 & 260 & Balco and Schaefer (2013) & 7698 & 269 \\
\hline Antarctic Peninsula (NE) & Sjögren/Boydell fjord (South) & 10-LAR-008-SJO & -64.264 & -59.077 & 303 & 53 & ${ }^{10} \mathrm{Be}$ & 6750 & 230 & Balco and Schaefer (2013) & 7538 & 238 \\
\hline Antarctic Peninsula (NE) & Sjögren/Boydell fjord (South) & 10-LAR-009-SJO & -64.264 & -59.077 & 303 & 53 & ${ }^{10} \mathrm{Be}$ & 7090 & 250 & Balco and Schaefer (2013) & 7917 & 256 \\
\hline Antarctic Peninsula (NE) & Sjögren/Boydell fjord (North) & 10-LAR-017-SJO & -64.195 & -58.980 & 116 & 116 & ${ }^{10} \mathrm{Be}$ & 4970 & 200 & Balco and Schaefer (2013) & 5555 & 202 \\
\hline Antarctic Peninsula (NE) & Sjögren/Boydell fjord (North) & 10-LAR-018-SJO & -64.195 & -58.980 & 116 & 116 & ${ }^{10} \mathrm{Be}$ & 4770 & 170 & Balco and Schaefer (2013) & 5327 & 175 \\
\hline Antarctic Peninsula (NE) & Sjögren/Boydell fjord (North) & 10-LAR-022-SJO & -64.197 & -58.978 & 74 & 74 & ${ }^{10} \mathrm{Be}$ & 4310 & 250 & Balco and Schaefer (2013) & 4805 & 278 \\
\hline Antarctic Peninsula (NE) & Sjögren/Boydell fjord (North) & 10-LAR-023-SJO & -64.199 & -58.978 & 39 & 39 & ${ }^{10} \mathrm{Be}$ & 3360 & 610 & Balco and Schaefer (2013) & 3743 & 688 \\
\hline Antarctic Peninsula (NE) & Sjögren/Boydell fjord (North) & 10-LAR-024-SJO & -64.199 & -58.978 & 39 & 39 & ${ }^{10} \mathrm{Be}$ & 3420 & 140 & Balco and Schaefer (2013) & 3811 & 149 \\
\hline
\end{tabular}




\begin{tabular}{|c|c|c|c|c|c|c|c|c|c|c|c|c|}
\hline Region & Site & Sample ID & Latitude & Longitude & $\begin{array}{l}\text { Altitude } \\
(\mathbf{m} \text { asl) }\end{array}$ & $\begin{array}{l}\text { Elevation } \\
\text { above } \\
\text { modern } \\
\text { ice }(m)\end{array}$ & $\begin{array}{l}\text { Cosmogenic } \\
\text { nuclide }\end{array}$ & $\begin{array}{c}\text { Published } \\
\text { age (yrs) }\end{array}$ & $\begin{array}{l}\text { Published } \\
\text { error (yrs) }\end{array}$ & Published source & $\begin{array}{l}\text { Recalc. age } \\
\text { (yrs) (NZ) }\end{array}$ & $\begin{array}{l}\text { Recalc. error } \\
\text { (yrs) (NZ) }\end{array}$ \\
\hline Antarctic Peninsula (W) & Moutonnée Valley (Alexander Is.) & MV2 & -70.862 & -68.439 & 650 & 630 & $\begin{array}{l}{ }^{10} \mathrm{Be},{ }^{26} \mathrm{Al} \\
\text { (weighted mean) }\end{array}$ & 25500 & 1700 & Bentley et al. (2006) & 31155 & 2180 \\
\hline $\begin{array}{l}\text { Antarctic Peninsula (W) } \\
\text { Antarctic Peninsula (W) }\end{array}$ & Moutonnée Valley (Alexander Is.) & $\begin{array}{l}\text { MV1 } \\
\text { MV5 }\end{array}$ & $\begin{array}{r}-70.862 \\
-70870\end{array}$ & -68.440 & 600 & 580 & ${ }_{26}^{26} \mathrm{Al}$ & 17300 & 1799 & Bentley et al. (2006) & 20963 & 2249 \\
\hline $\begin{array}{l}\text { Antararctic Peninsula (W) } \\
\text { Antarctic Peninsula (W) }\end{array}$ & $\begin{array}{l}\text { Moutonnée Valley (Alexander Is.) } \\
\text { Mount Holt (Alexander Is.) }\end{array}$ & L7.1.3. & $\begin{array}{l}-70.879 \\
-69.411\end{array}$ & $\begin{array}{l}-68.391 \\
-71.656\end{array}$ & 500 & 480 & ${ }^{10} \mathrm{Al}$ & $\begin{array}{l}11900 \\
21744\end{array}$ & $\begin{array}{l}1464 \\
1972\end{array}$ & $\begin{array}{l}\text { Bentley et al. (2006) } \\
\text { Johnson et al. (2012) }\end{array}$ & $\frac{14609}{26053}$ & $\frac{1837}{856}$ \\
\hline Antarctic Peninsula (W) & Mount Holt (Alexander Is.) & L7.1.1 & -69.412 & -71.671 & 580 & 340 & ${ }^{10} \mathrm{Be}$ & 15746 & 1422 & Johnson et al. (2012) & 18830 & 604 \\
\hline Mac.Robertson Land (EAIS) & North Masson Range & FM-NMas-4B-1 & -67.763 & 62.819 & 949 & 329 & $\begin{array}{c}{ }^{10} \mathrm{Be},{ }^{26} \mathrm{Al} \\
\text { (weighted mean) } \\
10 \mathrm{P}, 26\end{array}$ & 12000 & 700 & Mackintosh et al. (2007) & 16013 & 628 \\
\hline Mac.Robertson Land (EAIS) & North Masson Range & FM-NMas-18-3 & -67.752 & 62.830 & 786 & 266 & $\begin{array}{c}{ }^{10} \mathrm{Be},{ }^{26} \mathrm{Al} \\
\text { (weighted mean) }\end{array}$ & 10600 & 600 & Mackintosh et al. (2007) & 14439 & 465 \\
\hline Mac.Robertson Land (EAIS) & North Masson Range & FM-NMas-24-5 & -67.758 & 62.805 & 654 & 114 & $\begin{array}{l}{ }^{10} \mathrm{Be},{ }^{26} \mathrm{Al} \\
\text { (weighted mean) }\end{array}$ & 10000 & 600 & Mackintosh et al. (2007) & 13483 & 573 \\
\hline Mac.Robertson Land (EAIS) & North Masson Range & FM-NMas-23-6 & -67.755 & 62.802 & 642 & 102 & in situ ${ }^{14} \mathrm{C}$ & 9200 & 1000 & Mackintosh et al. (2007); White et al. (2011a) & & \\
\hline Mac.Robertson Land (EAIS) & North Masson Range & FM-NMas-20-7 & -67.758 & 62.783 & 635 & 55 & $\begin{array}{l}\text { in situ }{ }^{4} \mathrm{C} \\
10 \mathrm{P}\end{array}$ & 9200 & 1000 & Mackintosh et al. (2007); White et al. (2011a) & & \\
\hline Mac.Robertson Land (EAIS) & North Masson Range & FM-NMas-21-8 & -67.756 & 62.790 & 540 & -20 & (weighted mean) & 7700 & 400 & Mackintosh et al. (2007) & 10172 & 460 \\
\hline Mac.Robertson Land (EAIS) & Mt Henderson & FM-Hend-27-2 & -67.667 & 63.044 & 582 & 242 & $\begin{array}{c}{ }^{10} \mathrm{Be},{ }^{26} \mathrm{Al} \\
\text { (weighted mean) } \\
100\end{array}$ & 8900 & 600 & Mackintosh et al. (2007) & 11990 & 647 \\
\hline Mac.Robertson Land (EAIS) & Mt Henderson & FM-Hend-14-3 & -67.663 & 63.017 & 410 & 10 & $\begin{array}{c}{ }^{10} \mathrm{Be},{ }^{26} \mathrm{Al} \\
\text { (weighted mean) }\end{array}$ & 6600 & 700 & Mackintosh et al. (2007) & 8665 & 896 \\
\hline Prince Charles Mountains (EAIS) & Mt Stinear & Rym-173 & -73.015 & 65.905 & 993 & 393 & $\begin{array}{c}{ }^{10} \mathrm{Be},{ }^{26} \mathrm{Al} \\
\text { (weighted mean) }\end{array}$ & 21500 & 1200 & White et al. (2011b) & 25364 & 842 \\
\hline Prince Charles Mountains (EAIS) & Mt Stinear & Stin-117 & -73.046 & 66.481 & 521 & 361 & $\begin{array}{c}{ }^{10} \mathrm{Be},{ }^{26} \mathrm{Al} \\
\text { (weighted mean) } \\
100^{26}{ }^{26}\end{array}$ & 19200 & 1100 & White et al. (2011b) & 22750 & 847 \\
\hline Prince Charles Mountains (EAIS) & Mt Stinear & Stin-154a & -73.060 & 66.325 & 705 & 145 & $\begin{array}{c}\text { (weighted mean) } \\
\text { (wo }{ }^{10}{ }^{26} \mathrm{Al}\end{array}$ & 11700 & 700 & White et al. (2011b) & 13659 & 637 \\
\hline Prince Charles Mountains (EAIS) & Mt Stinear & Stin-154b & -73.059 & 66.326 & 700 & 140 & $\begin{array}{l}10 \mathrm{Be},{ }^{26} \mathrm{Al} \\
\text { (weighted mean) } \\
10 \mathrm{tan}\end{array}$ & 12200 & 700 & White et al. (2011b) & 14247 & 785 \\
\hline Prince Charles Mountains (EAIS) & Mt Stinear & Stin-147b & -73.002 & 66.535 & 320 & 120 & $\begin{array}{c}{ }^{10} \mathrm{Be},{ }^{26} \mathrm{Al} \\
\text { (weighted mean) }\end{array}$ & 10400 & 700 & White et al. (2011b) & 12178 & 720 \\
\hline Prince Charles Mountains (EAIS) & Mt Stinear & Stin-147c & -73.002 & 66.535 & 320 & 120 & $\begin{array}{c}10 \mathrm{Be} \\
10 \mathrm{Be}\end{array}$ & 10300 & 800 & White et al. (2011b) & 12138 & 774 \\
\hline Prince Charles Mountains (EAIS) & Mt Stinear & Stin-140 & -73.075 & 66.465 & 310 & 110 & (weighted mean) & 10000 & 700 & White et al. (2011b) & 11642 & 640 \\
\hline Prince Charles Mountains (EAIS) & Mt Stinear & Stin-6a & -73.006 & 66.490 & 535 & 95 & $\begin{array}{l}\text { (weighted mean) } \\
10 \mathrm{Be}\end{array}$ & 9200 & 600 & White et al. (2011b) & 11097 & 516 \\
\hline Prince Charles Mountains (EAIS) & Mt Stinear & Stin-8a & -73.075 & 66.288 & 670 & 85 & $\begin{array}{l}{ }^{10} \mathrm{Be},{ }^{26} \mathrm{Al} \\
\text { (weighted mean) }\end{array}$ & 10100 & 800 & White et al. (2011b) & 11985 & 542 \\
\hline Prince Charles Mountains (EAIS) & Mt Stinear & Sin- $8 \mathrm{~b}$ & -73.075 & 66.288 & 670 & 85 & ${ }^{10} \mathrm{Be}$ & 9800 & 800 & White et al. (2011b) & 11542 & 920 \\
\hline Prince Charles Mountains (EAIS) & Loewe Massif & LM-C12a & -70.536 & 67.800 & 329 & 229 & $\begin{array}{c}{ }^{10} \mathrm{Be},{ }^{26} \mathrm{Al} \\
\text { (weighted mean) } \\
10 \mathrm{D}\end{array}$ & 18900 & 1700 & White et al. (2011b) & 22062 & 1309 \\
\hline Prince Charles Mountains (EAIS) & Loewe Massif & LM-C8b & -70.546 & 67.961 & 242 & 162 & $\begin{array}{l}\text { (weighted mean) } \\
100^{10} \mathrm{Be}\end{array}$ & 15600 & 1100 & White et al. (2011b) & 18351 & 970 \\
\hline Prince Charles Mountains (EAIS) & Loewe Massif & LM-C3 & -70.517 & 68.004 & 156 & 116 & $\begin{array}{l}{ }^{10} \mathrm{Be},{ }^{26} \mathrm{Al} \\
\text { (weighted mean) } \\
10 \text { ) }\end{array}$ & 14500 & 1000 & White et al. (2011b) & 17039 & 934 \\
\hline Prince Charles Mountains (EAIS) & Loewe Massif & LM-C14b & -70.526 & 67.868 & 211 & 66 & $\begin{array}{l}{ }_{\text {(weighted mean) }} \\
\text { (we }{ }^{20} \mathrm{Al}\end{array}$ & 12100 & 900 & White et al. (2011b) & 14139 & 952 \\
\hline
\end{tabular}

Previously published surface-exposure ages have been recalculated using the New Zealand (NZ) production rate for comparison purposes, apart from in situ ${ }^{14} \mathrm{C}$ dates. Exposure ages used in Figure 7.14 are highlighted in bold. 


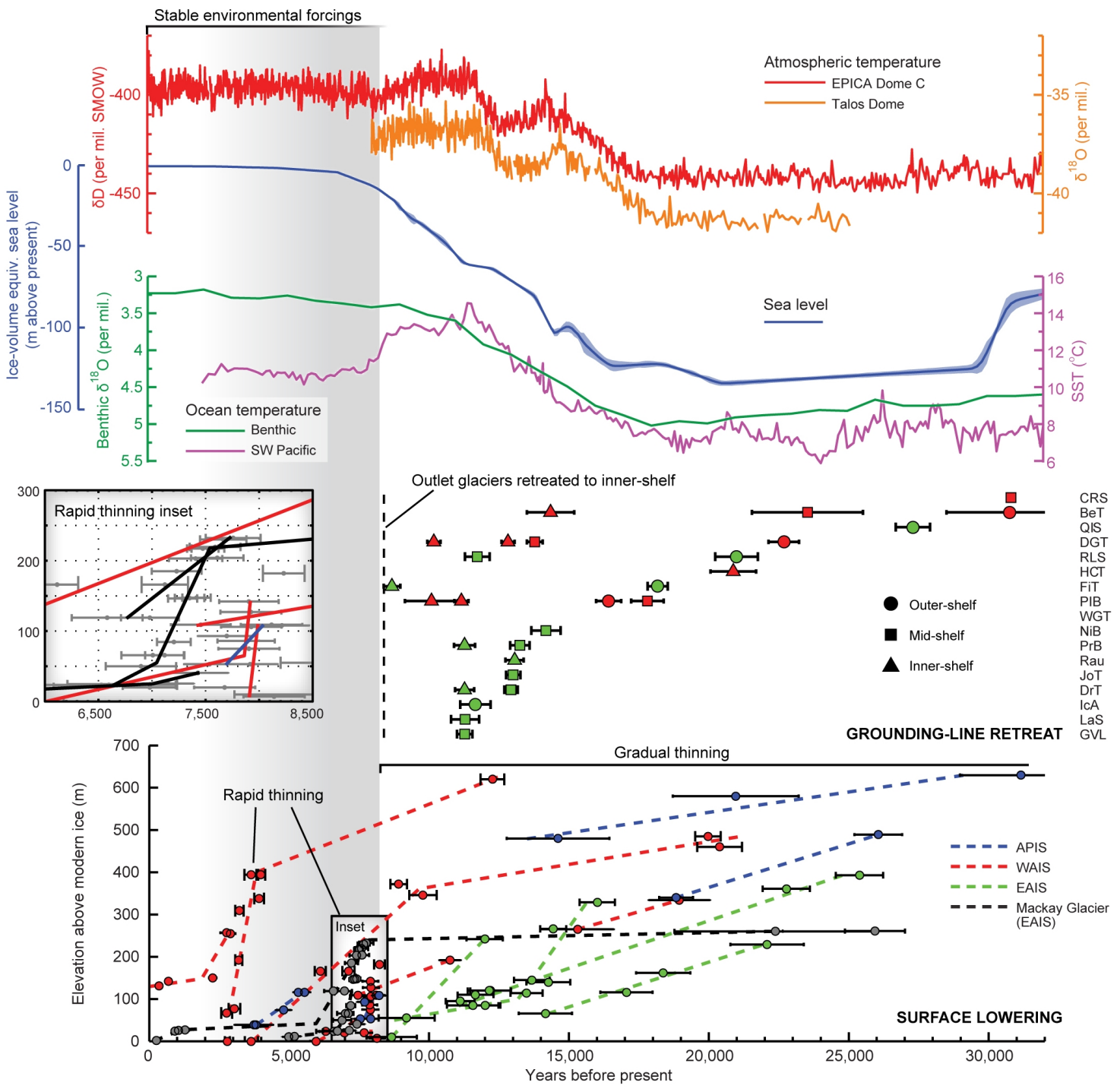

Figure 7.14 Circum-Antarctic evidence of ice sheet retreat and surface lowering compared to climate changes during the last deglaciation. Grounding-line retreat from EAIS (green) and WAIS (red) varies in timing between locations during the period 30-10 ka before present. Onshore data show that most ice surface lowering during this time was gradual. After grounding-lines retreated to the inner-shelf, rapid ice surface lowering is recorded at Mackay Glacier, as well as on parts of the APIS and WAIS between $\sim 8$ and $6 \mathrm{ka}$. Regional atmospheric temperature (red and orange lines; Jouzel et al. (2007) and Stenni et al. (2011), respectively), sea level (blue line, with $1 \sigma$; Lambeck et al., 2014) and ocean temperature (green and purple lines; Lisiecki and Raymo (2005) and Pahnke and Sachs (2006), respectively) were stable at this time, suggesting an alternative ice-dynamic factor to explain non-linear ice sheet thinning.

\subsubsection{Absence of significant environmental forcing at the time of rapid ice sheet thinning}

Circum-Antarctic empirical records of deglaciation reveal initial grounding-line retreat across the continental-shelf that was broadly synchronous with upstream thinning. Rapid thinning is then recorded in WAIS, APIS and EAIS during the early-mid Holocene, af- 
ter grounding-lines had retreated to the inner continental-shelf. This non-linear surface lowering response does not correspond in timing to increased atmospheric temperature (Jouzel et al., 2007; Stenni et al., 2011), sea level rise (Peltier, 2004; Stanford et al., 2011; Lambeck et al., 2014), or ocean temperature (Lisiecki and Raymo, 2005; Pahnke and Sachs, 2006) (Figure 7.14), likely ruling out enhanced surface melt (van den Broeke, 2005), floatation (Schoof, 2007) and sub-ice melt (Depoorter et al., 2013) driven instability. It is therefore highly possible that the widespread pulse of ice sheet thinning experienced during the Holocene was primarily driven by topographic effects of the inner-shelf rather than from significant environmental forcings.

\subsection{Conclusions}

This new data from Mackay Glacier reveals that accelerated ice sheet thinning at rates currently observed in Antarctica (Pritchard et al., 2009) may lead to hundreds of metres of ice surface lowering, which could persist for centuries to millennia. Model simulations show that this recorded thinning occurred as a consequence of marine ice sheet instability. This process may be underway today in larger Antarctic outlet glacier and ice stream systems (e.g. Favier et al., 2014; Joughin et al., 2014) and could ultimately lead to ice sheet collapse (Weertman, 1974; Mercer, 1978; Mengel and Levermann, 2014). The episode of rapid thinning documented at Mackay Glacier appears to have been widespread and demonstrates that under certain topographic conditions, delayed, non-linear ice sheet responses may still take place hundreds to thousands of years after initial ice sheet retreat. 


\section{Chapter 8}

\section{Synthesis}

Satellite observations reveal that some outlet glaciers in Antarctica are experiencing decadalscale dynamic thinning, terminus retreat and mass loss. The extent to which this behaviour occurs beyond natural variability remains unclear. It is therefore important to better understand the response of outlet glaciers to external forcings and internal instabilities.

In this thesis, I have used geomorphological, geochronological and numerical modelling techniques to investigate how two Transantarctic Mountain outlet glaciers changed their geometry and flow regimes under different climatic conditions. Four principle studies were conducted, directed by a set of specific research questions (Chapter 2.6, page 28). The outcomes of this work highlight future research directions, and contribute to the understanding of several overarching themes (Chapter 1.2, page 4).

\subsection{Principal conclusions}

1. What are the main environmental variables influencing the flow of Skelton Glacier during present and past climates?

The potential for outlet glacier mass loss is dependent on the specific dynamic response of the glacier to environmental forcing. Such a response can be assessed for different steady-state climates. In Chapter 4, the present-day and past dynamics of Skelton Glacier were investigated through the evaluation of observational datasets and numerical modelling.

Today, ice flow of Skelton Glacier is shown to be governed by its surface slope, as well as changes in valley width and large bed undulations, in common with other Transantarctic Mountain glaciers. In particular, bed topography provides the critical control on the along-flow response at Skelton Glacier, principally through its affect on ice thickness and the potential for basal melt. Threshold responses to subtle changes in environmental forcing and ice thickness are predicted at locations where bedrock ridges exist. A suite of sensitivity experiments identify the potential for enhanced ice deformation from increases in precipitation, however, greater ice volume loss is predicted in response to increases in atmospheric temperature, through its affect on basal sliding near the grounding-line.

Skelton Glacier is also simulated under past climates, informed by regional geological and palaeoclimate data. During both the Pliocene and Pleistocene, large changes 
occurred in the lower reaches of the glacier, predominantly from varying degrees of buttressing at the terminus. Under glacial and interglacial configurations, basal sliding and bedrock erosion is predicted in the overdeepened basins. Warmer-thanpresent atmospheric and oceanic temperatures during Pliocene interglacials likely led to a much shorter and thinner Skelton Glacier, with warm-based sliding that extended along most of its length.

\section{To what extent has Skelton Glacier undergone a stable or fluctuating geometry during the Late Cenozoic?}

Grounded ice in the Ross Sea has likely fluctuated in extent "in concert" with the West Antarctic Ice Sheet since before the Pliocene. Meanwhile, the East Antarctic Ice Sheet may have been a stable, cold-based feature. To address the uncertain configurations of Transantarctic Mountain outlet glaciers over this time, I applied surface-exposure dating in Chapter 5 to constrain past changes in the geometry of Skelton Glacier.

$\mathrm{A}{ }^{10} \mathrm{Be}$ exposure age from a fresh, glacially-transported erratic suggests that the lower reaches of Skelton Glacier were likely thicker at the Last Glacial Maximum (LGM). This is consistent with regional evidence that grounded ice in the Ross Sea acted to buttress the flow of Transantarctic Mountain outlet glaciers during glacial periods. The glacier then thinned to near-modern surface elevations by $\sim 5.8 \mathrm{ka} \mathrm{BP}$, lagging grounding-line retreat in the Ross Embayment by $>1,500$ years. Multiple isotope analysis $\left({ }^{26} \mathrm{Al}\right.$ and $\left.{ }^{10} \mathrm{Be}\right)$ records complex exposure histories of samples at nunataks in the central and upper névé, representing inheritance of cosmogenic nuclides from episodes of prior exposure. This is the result of negligible erosion $(<3.5$ $\mathrm{cm} \mathrm{ka}^{-1}$ ) under probable cold-based ice during glacial periods. These data record a total of at least $2.5 \mathrm{Ma}$ of burial and $>10 \mathrm{Ma}$ of exposure. Burial-exposure modelling of the complex histories suggests that the geometry of Skelton Glacier varied in response to fluctuating grounded ice in the Ross Embayment at orbital timescales since the Early Pliocene, and possibly the Mid-Miocene.

\section{How does the flow regime of Mackay Glacier vary temporally and spatially?}

Numerical ice sheet models have previously simulated polythermal regimes for large outlet glaciers but cold-based regimes for smaller glaciers in the past. New empirical data is required to better understand the past flow regimes of Transantarctic Mountain outlet glaciers. In Chapter 6, the long-term dynamics of Mackay Glacier were investigated through geomorphological assessment.

This evidence reveals that glaciers can both erode and preserve bedrock surfaces during the same glacial episode, with two apparent contrasting glacial regimes. 
Mackay Glacier likely experienced a widespread erosive regime prior to the Pleistocene and a polythermal glacier regime during the LGM. Basal erosion is shown to be controlled primarily by ice thickness, with sufficiently deep glacier trunks able to accommodate warm-based ice during glacial maximum configurations. This potential for warm-based ice and basal erosion reduces upstream and at the glacier flanks where ice is thinner. Based on the geomorphological assessment, an LGM ice surface profile is reconstructed. This is consistent with regional evidence of thickening in the lower reaches of Mackay Glacier, but identifies an ice surface $>100 \mathrm{~m}$ higher than previous estimates. These new interpretations suggest that coarsely-gridded ice sheet models overestimate ice thickness in this area at the LGM and underestimate the potential for warm-based ice in small outlet glaciers.

\section{What was the style and mechanism of thinning at Mackay Glacier during the last deglaciation?}

The brevity of modern observations limits our understanding of the processes driving deglaciation. However, geological data can extend the timescale of ice sheet observations, providing rates, durations and magnitudes of past ice loss. In Chapter 7, deglaciation following the LGM was constrained with ${ }^{10} \mathrm{Be}$ surface-exposure dating at Mackay Glacier. The mechanism responsible for glacier thinning was then investigated with idealised modelling experiments.

Samples collected at two nunataks, across four "dipstick" transects, reveal glacier thinning of $>260 \mathrm{~m}$ between the LGM and $\sim 200$ years BP. Ice surface lowering was initially gradual, however an episode of rapid thinning is then recorded at $\sim 6.8-7.8 \mathrm{ka} \mathrm{BP}$, during a period of relative climatic and oceanic stability. This accelerated surface lowering occurred at a rate commensurate with modern observations of rapid ice sheet thinning, persisted for $>420$ years, and resulted in $>180 \mathrm{~m}$ of ice loss. Numerical modelling indicates that ice surface drawdown resulted from 'marine ice sheet instability'. Simulated retreat and ice flux accelerates when the grounding-line encounters the reverse bed slope of a deep glacial trough on the inner continental shelf, consistent with the surface-exposure chronology. Based on this evidence and the timing of deglaciation recorded elsewhere, I argue that widespread thinning of the Antarctic ice sheet margin during the early to mid-Holocene may have resulted from ice-dynamic feedbacks rather than direct climate forcing. 


\subsection{Recommendations for future work}

The outcomes of this thesis provide new insights into outlet glacier behaviour and the application of surface-exposure dating and numerical modelling. This work also highlights several research areas that deserve further attention.

\section{Complex bed topography of small outlet glaciers is under-represented in continental-} scale ice sheet models. Flowline modelling of Skelton Glacier, using high resolution aerially-derived ice thickness radar data, identifies the important roles of narrowing valley width and undulating bed topography in modulating ice flux. In particular, this work highlights how small changes in ice thickness at a bedrock ridge can lead to basal melt, sections of continuous basal sliding and, in turn, enhanced ice discharge. Such subtle, but important, changes in bed topography are not accurately represented in more coarselygridded bed topography data (Fretwell et al., 2013). These data are used in continentalscale ice sheet models to simulate potential mass loss (e.g. Mengel and Levermann, 2014) and past ice sheet dynamics (e.g. Golledge et al., 2013; Gomez et al., 2013). Geomorphological assessment at Mackay Glacier demonstrates how such models fail to predict basal melt and erosion in relatively small outlet glaciers with deep central trunks. Together, work at Skelton and Mackay Glaciers indicate that ice sheet models need to include more accurate and higher resolution bed topography data in order to adequately capture basal sliding and ice discharge at small outlet glaciers that are ubiquitous around the high-relief Antarctic margins. This is currently limited by the incomplete coverage of ice depth radar surveys and the high computational requirements to run models with finer grids. Higher resolution bed topography would also need to be matched with similarly fine detail in model forcings and basal substrate data that are used in ice sheet models.

\section{Potential propagation of outlet glacier dynamic thinning should be investigated with surface-exposure dating. Chronologies developed at Mackay Glacier were able to record} the magnitude and duration of rapid thinning in the lower reaches, the driver of which I then demonstrate with numerical modelling. Surface-exposure transects at several nunataks also suggest differences in the magnitude and timing of rapid thinning along Mackay Glacier. Such potential propagation effects have been simulated previously at outlet glaciers (e.g. Nick et al., 2009), but geological data are required to constrain this dynamic response on greater than decadal timescales. Capturing not only the mechanism and downstream response of rapid dynamic thinning, but also the extent to which this was propagated upstream is critically important for improving our understanding of the potential ice discharge from a glacier system following a perturbation near the terminus. We cannot decipher the full extent of centennial to millennial scale dynamic thinning without surface-exposure dating. It is therefore recommended that large outlet glacier systems (longer than Mackay Glacier) should be targeted, with a central line of nunataks extending upstream suitable for surface-exposure dating with "dipstick" transects. 
Cosmogenic nuclide production rates require improved time-dependent and Antarcticspecific constraints. Surface-exposure dating carried out at Skelton Glacier identifies how the production rate of ${ }^{10} \mathrm{Be}$, and therefore ${ }^{26} \mathrm{Al}$, appears to have been at least double that of today on average in the past. Such an offset cannot be explained by the current understanding of temporal variations in production rates. This work highlights the significant uncertainty associated with applying surface-exposure dating on the scale of 100,000 s to millions of years, beyond the times when the production rate is directly constrained at calibration sites. Furthermore, several recently published calibration sites (e.g. Putnam et al., 2010; Kaplan et al., 2011; Young et al., 2013) suggest a modern production rate substantially lower than the previously determined global production rate (Balco et al., 2008). Currently, no calibration sites exist within $\sim 3000-4000 \mathrm{~km}$ of Antarctica, and therefore the application of surface-exposure dating here relies on the choice of production rate and spatial scaling (Stone, 2000). The effect of different production rates is demonstrated at Mackay Glacier as a $\sim 1$ ka exposure age offset during the midHolocene (Figure 7.5). While not affecting outcomes in this thesis, such production rate uncertainty could prevent detailed assessment of outlet glacier behaviour when a surfaceexposure chronology is compared with other records, for example, to radiocarbon-dated grounding-line retreat. Unfortunately, the determination of an Antarctic-specific production rate requires a large geomorphic event (e.g. landslide) and reliable independent age control, which are difficult to identify and obtain in Antarctica's terrestrial environment.

\subsection{Overarching research contribution}

Research carried out for this thesis has contributed new understanding that is pertinent to several overarching themes.

- Changes in the geometry and dynamics of Transantarctic Mountain outlet glaciers during the Pliocene and Quaternary

Changes in the geometry and dynamics of outlet glaciers are inherently linked to the prevailing climatic conditions, and it is therefore important to understand the steady-state behaviour of such outlet systems under a range of past climates. For Transantarctic Mountain outlet glaciers, the extent to which their geometries have undergone long-term stability with the East Antarctic Ice Sheet (e.g. Sugden et al., 1993) or orbitally-paced fluctuations with West Antarctic grounded ice in the Ross Embayment (e.g. Naish et al., 2009) has remained unclear. Surface-exposure dating at Skelton Glacier shows that these outlets have likely fluctuated between interglacial and glacial states since the Early Pliocene, and possibly the Miocene. 
Glacier modelling indicates that during the early, warmer-than-present interglacials, basal sliding probably extended along most of the glacier, facilitating basal erosion in the central trunk. Geomorphological signatures at Mackay Glacier record an erosive glacial regime, likely under warmer-than-present conditions but with an ice surface higher than today. Under thicker configurations, cold-based, non-erosive ice was prevalent at higher elevation nunataks. During the Pleistocene, numerical modelling and surface-exposure dating at Skelton Glacier suggests that the potential for basal melt and erosion is diminished. However, in the deep glacier trunks and near the terminus where ice was sufficiently thick, geomorphological assessment at Mackay Glacier highlights how basal erosion and probable warm-based sliding was possible during glacial periods.

In summary, these outcomes suggest that predominantly warm-based outlet glaciers, sourced from a stable cold-based East Antarctic interior (Siegert et al., 2008), facilitated selective erosion (Jamieson et al., 2010) and underwent high magnitude fluctuations in ice thickness at orbital timescales during the Pliocene. By the Pleistocene, the glacial regimes had changed from predominantly warm-based (sub-polar) to cold-based (polar), consistent with sedimentological evidence (McKay et al., 2009). These outlet glaciers continued to fluctuate between glacial and interglacial states during the Pleistocene, and warm-based sliding was still possible where ice was sufficiently thick.

\section{- Ice sheet thickness in the western Ross Sea at the Last Glacial Maximum, and possible contribution to 'meltwater pulses'}

Understanding the potential contribution of Antarctic ice sheets to rapid future sea level rise requires an assessment of past ice loss events (Church et al., 2013). Deglaciation following the LGM produced several such events, evident in sea level records as 'meltwater pulses'. The most significant of these was MWP-1A, but its source remains uncertain (Carlson and Clark, 2012). Contributions likely came from Antarctica (Weber et al., 2014; Golledge et al., 2014b), however for Antarctic ice sheets to be the primary source, they must have contained at least $7 \times 10^{6} \mathrm{~km}^{3}$ more ice at the LGM than at present. This is approximately 1.2-2 times that determined from ice sheet models (e.g. Mackintosh et al., 2011; Whitehouse et al., 2012; Golledge et al., 2014b).

In the Ross Sea sector at the LGM, these ice sheet models simulate a groundingline near the continental-shelf edge, beyond that recorded by geological data (e.g. Shipp et al., 1999; Anderson et al., 2014), and ice surface elevations along the front of the Transantarctic Mountains that are several hundred metres higher than what terrestrial evidence suggests (e.g. Bockheim et al., 1989; Orombelli et al., 1990; Denton and Marchant, 2000; Denton and Hughes, 2000; Todd et al., 2010). New 
geomorphological and geochronological data at Mackay Glacier reveal an LGM ice surface $>100 \mathrm{~m}$ higher than previous estimates (e.g. Denton and Hughes, 2000), but still lower than that simulated by ice sheet models. On this basis, I suggest that current models overestimate ice sheet extent in this region at the LGM, and therefore it is unlikely that grounded ice in the western Ross Sea could have contributed significantly to MWP-1A. Further work at other outlet glaciers is needed to better constrain ice thickness in this region at the LGM and the timing of subsequent deglaciation. In particular, research should focus further north, at outlet glaciers in Northern Victoria Land. This is near to the maximum extent of the LGM ice sheet (Anderson et al., 2014), and therefore the onset of deglaciation in the western Ross Sea should be recorded here first, reflected in the past ice thickness of adjacent outlet glaciers.

- Onset of ice surface lowering following the Last Glacial Maximum and the dynamic processes facilitating deglaciation

Deglaciation from the LGM to present-day occurred in response to the last major period of carbon dioxide increase and atmospheric warming on Earth, and therefore provides an opportunity to better understand the response of ice sheets to enhanced warming. Records of deglaciation suggest that initial grounding-line retreat of the Antarctic ice sheet was broadly synchronous with upstream thinning (Bentley et al., 2014). At Mackay Glacier, this is recorded at 18-22 ka BP when the groundingline of ice in the western Ross Sea was still located on the outer continental-shelf (Cunningham et al., 1999; Domack et al., 1999). At this time, the primary driver of ice sheet retreat and surface lowering was probably global sea level rise, after initial deglaciation of Northern Hemisphere ice sheets (Clark et al., 2009). Following $\sim 17 \mathrm{ka}$, once the Atlantic Meridional Overturning Circulation had recovered from a Northern Hemisphere meltwater flux (Heinrich Event 1) (Liu et al., 2009), the southwest Pacific Ocean began to warm (Pahnke and Sachs, 2006). Together with rising sea level, this may have driven ice surface lowering in East Antarctica (Mackintosh et al., 2011), and together with enhanced upwelling of the Circumpolar Deep Water current, may have caused substantial grounding-line retreat of central Antarctic embayments (Golledge et al., 2014b). Evidence at Mackay Glacier, however, suggests possible surface lowering that was gradual and relatively small in magnitude over this time, although an episode of thinning and then thickening prior to the Holocene cannot be ruled out.

During the Holocene, atmospheric temperature, sea level and sea surface temperatures approached present-day values. Grounded ice in the western Ross Sea continued to retreat, through the Drygalski Trough at $\sim 11$ ka BP (Licht et al., 1996) to just north of Ross Island by $\sim 10 \mathrm{ka}$ BP (Mckay et al., 2008). This preceded rapid thinning of Mackay Glacier at $\sim 6.8-7.8 \mathrm{ka} \mathrm{BP}$, which was driven by local 
grounding-line retreat into an overdeepened trough. Rapid surface lowering occurred elsewhere in Antarctica during the early to mid-Holocene (e.g. Balco and Schaefer, 2013; Bentley et al., 2010; Fogwill et al., 2012; Johnson et al., 2014) where grounding-lines occupied troughs on the inner-shelf. It is therefore probable that this retreat was also driven by 'marine ice sheet instability' rather than direct climate forcing. Grounded ice in the western Ross Sea then retreated past Skelton Glacier sometime before $\sim 5.8 \mathrm{ka} \mathrm{BP}$, until it reached its near-present configuration at $\sim 2$ ka BP (Hulbe and Fahnestock, 2007). Following grounding-line retreat, surface lowering at Skelton Glacier largely ceased within a couple of thousand years and at Mackay Glacier was gradual until at least $\sim 200$ years BP.

\section{- Sensitivity of Transantarctic Mountain outlet glaciers to environmental forcing and topographic feedbacks}

The sensitivity of outlet glaciers to external forcing and internal feedbacks is relevant for understanding present-day ice sheet mass loss. An important goal in this thesis was to better understand the dynamic response of Transantarctic Mountain outlet glaciers. Model sensitivity experiments at Skelton Glacier identified that changes in precipitation and atmospheric temperature can cause significant adjustments in the magnitude and extent of ice deformation and basal sliding, respectively. Outside of potential buttressing effects, the greatest mass loss was predicted from increased atmospheric temperature, which resulted in enhanced sliding and ice discharge. The most dynamic changes occurred in the lower reaches of the glacier, where flow is heavily modulated by valley width and bed topography. Importantly, this work identifies a threshold response from subtle changes in ice thickness and atmospheric temperature, which highlights the sensitivity of these outlet glaciers to environmental forcing in combination with topographic controls.

The influence of catchment topography on ice flux is best demonstrated at Mackay Glacier. Here, dynamic glacier thinning as a result of 'marine ice sheet instability' (e.g. Weertman, 1974) is identified with geological data and numerical modelling. Significant ice surface drawdown resulted from accelerated grounding-line retreat over a reverse bed slope, irrespective of the environmental forcing. Such behaviour highlights the potential for climatically-decoupled ice loss in outlet glaciers that occupy overdeepened basins in the absence of substantial downstream buttressing. In summary, Transantarctic Mountain outlet glaciers are susceptible to mass loss as a result grounding-line retreat through overdeepenings, an effect which could be enhanced by increased atmospheric temperatures and corresponding basal sliding.

\section{- Implications for observed and projected ice sheet changes}

Antarctic ice sheets are currently experiencing atmospheric warming (Steig et al., 
2009) and are undergoing mass loss (Shepherd et al., 2012). Satellite observations reveal termini retreat (Miles et al., 2013) and decadal-scale dynamic thinning (Pritchard et al., 2009) of many outlet glaciers, some of which are grounded in basins below sea level and are, therefore, considered vulnerable to 'marine ice sheet instability' (e.g. Favier et al., 2014; Mengel and Levermann, 2014). My work at Mackay Glacier demonstrates that the rapid ice sheet loss observed today may persist for centuries to millennia, leading to many hundreds of metres of ice loss.

Under projected warming, some Antarctic glaciers are anticipated to melt (Davies et al., 2014) and increase discharge (Winkelmann et al., 2012). In the southern Victoria Land portion of the Transantarctic Mountains, outlet glaciers are shown in this thesis to lose mass primarily in response to atmospheric warming opposed to changes in precipitation, via enhanced sliding at the bed. Under these conditions and in the absence of a buttressing ice-shelf, grounding-lines may retreat into overdeepenings, which are common near modern grounding-lines (e.g. Golledge et al., 2014a). If future greenhouse gas concentrations were to increase along the RCP8.5 pathway, Antarctic temperatures may warm to those of the Pliocene (Masson-Delmotte et al., 2013). An ice sheet in equilibrium with these warmer conditions would look very different to that of today, with shorter outlet glaciers and higher levels of basal sliding. Such an ice sheet would be even more susceptible to enhanced mass loss and would likely contribute substantially to sea level rise (Church et al., 2013). 


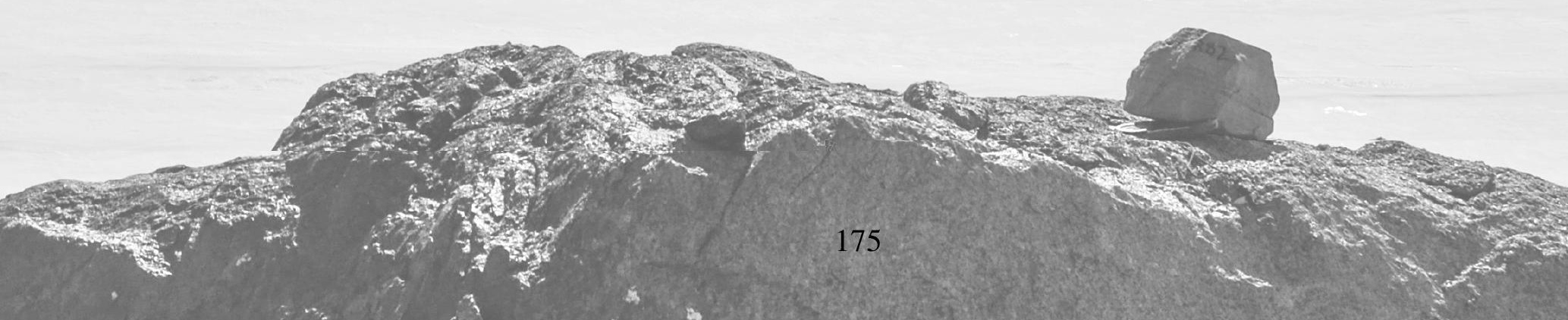




\section{Appendix A}

\section{Sample collection \& preparation}

\section{A.1 Field work}

Field work was undertaken over two field seasons. Geomorphological mapping and sample collection for surface-exposure dating was carried out at Skelton Glacier in OctoberNovember 2011 and at Mackay Glacier in January 2013.

The collection of samples was accompanied by measurements of latitude, longitude and altitude using a handheld Trimble GeoExplorer GPS ( $\pm<0.5 \mathrm{~m}$ precision), in addition to local and regional topographic shielding and sample surface dip/orientation using a compass and clinometer. Sample surface characteristics (e.g. weathering, glacial abrasion), the substrate underlying the sample (e.g. compacted till, bedrock), local geomorphic features (e.g. ice-moulded bedrock, rockfall debris), and sample size and lithology were also documented.

Samples collected included cobbles, boulders and bedrock surfaces. The top of the sample surface was labelled in the field. Bedrock and large cobbles/boulders were collected and sub-sampled with a hammer and chisel, and additionally with a battery-powered circularsaw (angle-grinder) at Mackay Glacier.

\section{A.2 Sample preparation}

On return to Victoria University of Wellington, characteristics of the samples were evaluated in order to select the most reliable surface of continuous, unmodified exposure (Figure A.1). Subsequently, the primary surface was sub-sampled with a circular-saw and the thickness was measured $(\sim 2-8 \mathrm{~cm})$.

This material was then crushed with a Boyd jaw-crusher to $<500$ microns and sieved to retain the 250-500 micron fraction. Each sample was initially cleaned using a Frantz Isodynamic separator (at $\sim 0.5 \mathrm{Amps}$ and a $10^{\circ}$ tilt) to remove the magnetic component. One granite sample required additional heavy liquid separation, using lithium tungstate liquid that was diluted to $2.60 \mathrm{~g} / \mathrm{mL}$.

The resulting sample consists of near-pure $(\sim 98 \%)$ quartz, which is further cleaned and etched prior to separation of cosmogenic nuclides. 

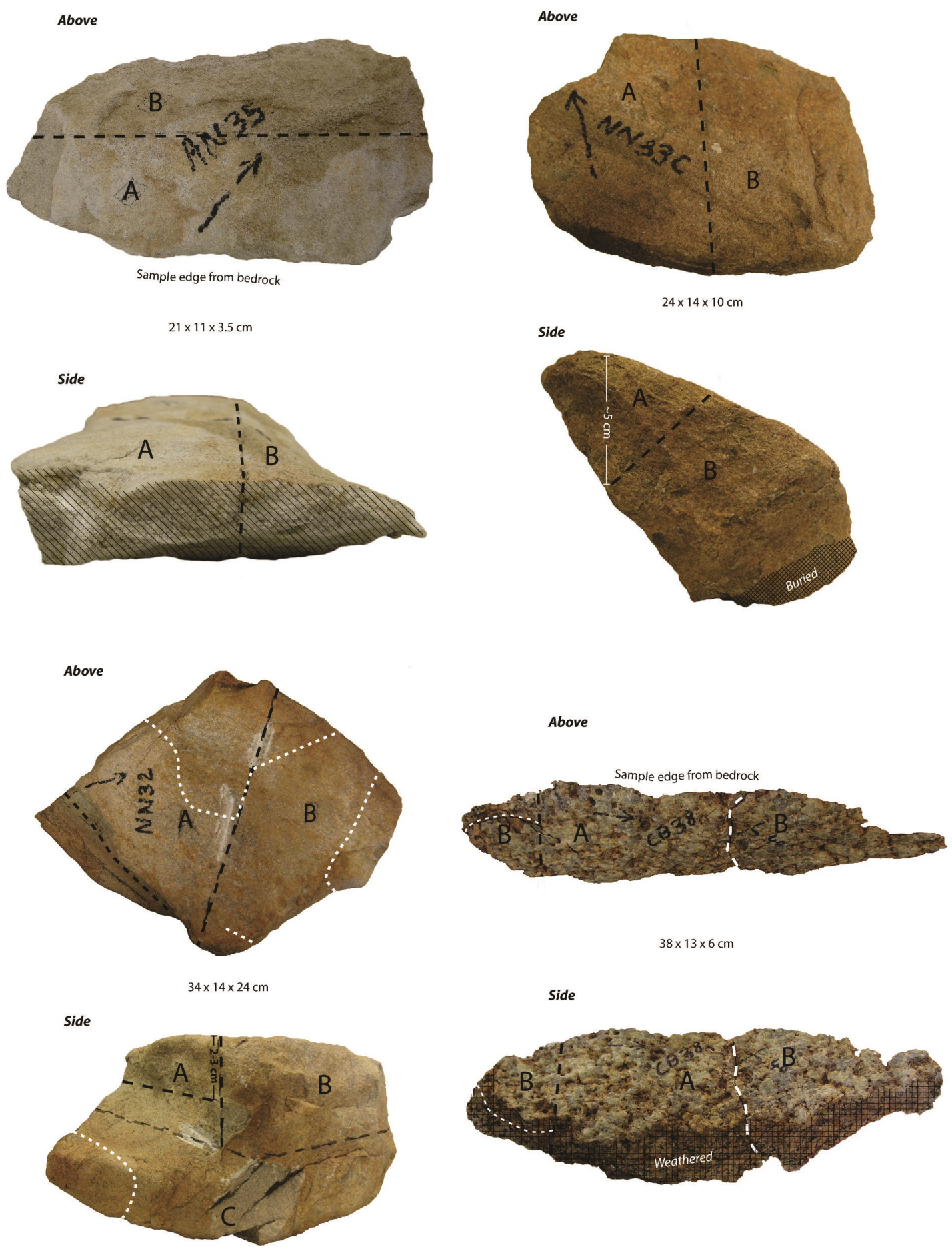

Figure A.1 Examples of collected samples assessed for surface-exposure dating. First, parts were identified where: 1) an edge was sub-sampled from bedrock or a larger boulder in the field (marked with diagonal lines), 2) the sample was buried (diagonal hatch) or heavily weathered (vertical-horizontal hatch) in the field, and 3) the sample broke apart during collection (dashed white line). Second, sections of each sample were prioritised (first, A; second, B), with a preference for top, glacially-eroded, unweathered, unburied surfaces. Corresponding circular-saw lines (black dashed) and additional resulting sub-sample boundaries (white dotted line) are marked. The thickness of the final primary sub-sample is measured from the top surface, later used to calculate the surface-exposure age of the sample. An arrow was drawn on the sample in the field to show magnetic north on the top surface. 


\section{Appendix B}

\section{Quartz preparation and separation of cosmogenic Be and Al}

\section{B.1 Initial quartz separation}

Sieve dry sample to appropriate size fractions

If iron particle content is high: clean sample with hand magnet

If magnetic mineral content is high: Frantz magnetic separation

Take weight, sample should be $>200 \mathrm{~g}$ (dependant on Quartz content)

Wash sample with deionised water

Add $10 \% \mathrm{HCl}\left(36 \% \mathrm{HCl}: \mathrm{H}_{2} \mathrm{O}=1: 3\right)$ to the sample (in an open container)

Notes: remember to add acid to water $(\sim 330: 660 \mathrm{ml}) ;<150 \mathrm{~g}$ of sample per container

Repeat if the carbonate content is high (e.g. foaming)

Add sample and $\sim 250 \mathrm{ml} 10 \% \mathrm{HCl}$ to 11 bottles

Place on hotdog rollers for $\sim 1$ day @ $60^{\circ} \mathrm{C}\left(\sim 50^{\circ} \mathrm{C}\right.$ on machine $)$

Wash with deionised/distilled water

Note: tip liquid of sample bottles into bucket, wash sample 3 times until water is clear

Repeat step if solution is very dirty (yellow)

\section{B.2 Quartz cleaning and etching (HF version)}

No more than $80 \mathrm{~g}$ of a dirty sample or $120 \mathrm{~g}$ of a clean (quartz-rich) sample should go in each 1 litre HDPE bottle. All shaking, rolling or ultrasonic should be done heated:

Make 5\% HF solution: 
Per 1 litre: $100 \mathrm{ml}$ conc. ( 48\%) HF (analytical grade), $10 \mathrm{ml}$ conc. ( $~ 68 \%)$ $\mathrm{HNO}_{3}, 890 \mathrm{ml}$ deionized $\mathrm{H}_{2} \mathrm{O}$

$\mathrm{H}_{2} \mathrm{O}$ Or per sample bottle (max. $300 \mathrm{ml}$ ): $\sim 37 \mathrm{ml}$ conc. $\mathrm{HF}, \sim 5 \mathrm{ml} \mathrm{HNO}_{3}, \sim 250 \mathrm{ml}$ Note: add water, then $\mathrm{HNO}_{3}$, then $\mathrm{HF}$ to sample

Add $500 \mathrm{ml} \mathrm{5 \%} \mathrm{HF} \mathrm{to} \mathrm{sample} \mathrm{and} \mathrm{spin} \mathrm{on} \mathrm{heated} \mathrm{rollers} \mathrm{for} \sim 1$ day

\section{Note: Turn off rollers overnight}

Wash with deionized/distilled water

Note: wash at least $4 \mathrm{x}$, dumping into waste bucket; calcium carbonate should then be added to bucket (binding with fluoride) and soda ash (neutralising acid)

Add $500 \mathrm{ml} 5 \%$ HF to sample and shake for 2 days

Wash with deionised/distilled water

Add $500 \mathrm{ml} 5 \%$ HF to sample and shake for 2 days

Wash with deionised/distilled water

Repeat until sample is pure quartz (either visual inspection or $<5 \%$ weight change)

Wash with Millipore $\mathrm{H}_{2} \mathrm{O}$ until $\mathrm{pH} \sim$ neutral, then dry and weigh sample

Note: dry in oven in bowls with filter paper

If the samples are still impure, use heavy liquid separation or hand picking to remove impurities. 


\section{B.3 Final Quartz Leach}

\section{SAMPLE DECOMPOSITION}

IMPORTANT: From this point on, use only p.a. grade or cleaner acids only, and use distilled or Millipore water

Note: clean beakers prior to use (enough large $(240 \mathrm{ml}$ or $90 \mathrm{ml})$ Savillex beakers for dissolution and small (22ml) Savillex beakers for outputs); 1) wipe and rinse, 2) clean with $\mathrm{HCl}\left(\sim 1: 3\right.$ with $\left.\left.\mathrm{H}_{2} \mathrm{O}\right), 3\right)$ with $\mathrm{HNO}_{3}(\sim 2: 5)$, and 4) with $7 \mathrm{M} \mathrm{HF}(\sim 1: 4)$, just covering the base each time and rinsing with Millipore water in between.

Take the precise weight of a $90 \mathrm{ml}$ or $240 \mathrm{ml}$ Savillex screwtop jar and lid (maximum $\sim 50 \mathrm{~g}$ quartz in $90 \mathrm{ml}$ jar and maximum $\sim 150 \mathrm{~g}$ in $240 \mathrm{ml}$ jar) (if using the quick dissolution, only use the largest Savillex jars)

Add $7 \mathrm{M} \mathrm{HF}$ ( $48 \%$ conc. $\mathrm{HF}(28 \mathrm{M})$ : Millipore $\mathrm{H}_{2} \mathrm{O}=1: 3$ ) till the sample is covered by $\sim 5 \mathrm{~mm}$ excess liquid

Heat $\sim 1$ hour (maximum) @ $120^{\circ} \mathrm{C}$ with lid

Allow to cool and wash with Millipore $\mathrm{H}_{2} \mathrm{O}$

\section{Note: $4 \mathrm{x}$ rinses per sample}

Add Aqua Regia (conc. $(14 \mathrm{M}) \mathrm{HNO}_{3}$ : conc. $\left.(12 \mathrm{M}) \mathrm{HCl}=1: 3\right)$ till the sample is covered by $\sim 5 \mathrm{~mm}$ excess liquid

Note: to make batch for $\sim 13$ samples ( $\sim 500 \mathrm{ml}$ of Aqua Regia), add $450 \mathrm{ml} \mathrm{HCl}$ and $150 \mathrm{ml} \mathrm{HNO}_{3}$

Leave uncovered until all brown gas has gone, $\sim 30$ minutes

Heat with lid $\sim 1$ hour minimum @ $120^{\circ} \mathrm{C}$

Note: if leaving for a long period (i.e. overnight), tighten lid and leave off hotplate

Allow to cool and wash thoroughly with Millipore $\mathrm{H}_{2} \mathrm{O}$

Repeat 4-5 times

Dry sample on hotplate (over $1 / 2$ day or overnight)

Allow to cool and take the precise weight (including Savillex jar and lid) 


\section{B.4 Carrier addition and sample dissolution}

The amount of carrier added will depend on the AMS used

$0.15 \mathrm{mg} \mathrm{Be}(\mathrm{ETH})$

$0.4 \mathrm{mg}$ Be (GNS Science)

$0.2 \mathrm{mg}$ Be (East Kilbride)

(i.e. if the carrier conc. $=405 \mathrm{ppm}$ then $0.37 \mathrm{~g}$ of carrier solution $=0.15 \mathrm{mg} \mathrm{Be}$, or if carrier conc. $=375 \mathrm{ppm}$ then $0.45 \mathrm{~g}=0.15 \mathrm{mg} \mathrm{Be}$ )

Take the precise weight of carrier added (to $0.000 \mathrm{~g}$ or better)

Notes: pour approx. carrier to be used into a cleaned bottle, add carrier to sample using $100-1000 \mu \mathrm{L}$ pipette, measure mass loss from the carrier (take precise weight and then tare after each).

\section{B.5 Dissolution}

\section{B.5.1 Version 1: slow and steady}

The stochiometric reaction for quartz dissolution is: $\mathrm{SiO}_{2}+4 \mathrm{HF}->\mathrm{SiF}_{4}+2 \mathrm{H}_{2} \mathrm{O}$

(e.g. you will need $116 \mathrm{ml} 28 \mathrm{M} \mathrm{HF}$ to dissolve $50 \mathrm{~g}$ quartz, however, in practice you will need a bit more)

Add 28M HF (remember p.a. quality or better) in increments till the sample is covered with $\sim 5 \mathrm{~mm}$ excess liquid

The first couple HF additions may generate a strong exothermic reaction, so be careful

Allow to react unheated for $\sim 15$ minutes

Heat without lid @ $120^{\circ} \mathrm{C}$, eventually evaporating to dryness

Repeat until all quartz is dissolved

Note: after the $2^{\text {nd }}$ or $3{ }^{\text {rd }} \mathrm{HF}$ addition, the reaction should be less volatile, and you can add HF up to twice the quartz volume.

Carefully remove from hotplate and allow to cool 
Note: static electricity that can cause sample flakes to jump out of the beaker wetting your gloves a bit and/or placing wet paper towels beneath the beakers can help reduce this effect.

\section{B.5.2 Version 2: fast and furious}

Reminder: use only large (240ml or larger) Savillex beakers for this procedure

Slowly and incrementally add $2 \mathrm{x}$ the stochiometric amount of HF

CAREFUL! Monitor temperature!

After initial reactions, close the beakers and place them into a cold water bath overnight

Remove beakers from water bath and heat @ $120^{\circ} \mathrm{C}$ closed until all quartz is dissolved

Remove beakers from the hotplate and allow to cool

Carefully remove the lid and heat on the hotplate @ $\sim 120^{\circ} \mathrm{C}$ to dryness

Note: if a white fluoride has built on the lid or beaker, rinse this into the beaker with Millipore water and evaporate.

Carefully remove from hotplate and allow to cool

Note: static electricity that can cause sample flakes to jump out of the beaker wetting your gloves a bit and/or placing wet paper towels beneath the beakers can help reduce this effect.

\section{B.6 Sample conversion}

\section{B.6.1 Sample conversion (with Al-chemistry)}

(To be done if Al will/might be measured)

Add $20 \mathrm{ml}$ Aqua Regia $\left(6 \mathrm{ml} 15 \mathrm{M} \mathrm{HNO}_{3}\right.$ and $\left.12 \mathrm{ml} 6 \mathrm{M} \mathrm{HCl}\right)$

For 10x samples with 18ml Aqua Regia: $\mathrm{HNO}_{3} \sim 70: 130 \mathrm{ml} \mathrm{HCl}$

Heat until all residue dissolved and evaporate gently

Note: takes $\sim 1 / 2$ day - do not burn sample! 
Add $10 \mathrm{ml} 6 \mathrm{M} \mathrm{HCl}$

Transfer into cleaned $10 \mathrm{ml}$ (or 11/15 ml) centrifuge tubes (when still warm)

Note: clean tubes with weak $\mathrm{HCl}$ and then Millipore $\mathrm{H}_{2} \mathrm{O}$ rinse

Centrifuge for 5 minutes @ 3000 rpm

Note: clean $11 \mathrm{ml}$ or $15 \mathrm{ml}$ centrifuge tubes and $60 \mathrm{ml}$ bottle ( 1 of each per sample), with at least a weak $\mathrm{HCl}$ and then Millipore $\mathrm{H}_{2} \mathrm{O}$ rinse

Label cleaned centrifuge tubes for Al-TSS, place empty tube on balance, zero balance

Label cleaned $60 \mathrm{ml}$ bottle for Al-aliquot, place on second (less precise) balance, take bottle weight, then zero balance

Transfer sample solution into centrifuge tube, leave undissolved residue behind, take weight of TSS (total sample solution)

Take $250 \mu \mathrm{l}$ aliquot, transfer into $60 \mathrm{ml}$ bottle, take weight of $\mathrm{Al}$ aliquot

Add $5 \mathrm{ml} 3 \mathrm{M} \mathrm{HNO}_{3}$ to $\mathrm{Al}$ aliquot (storage in strong acid to prevent adsorption of $\mathrm{Al}$ )

Before OES measurement: dilute to $0.3 \mathrm{M} \mathrm{HNO}_{3}$ by addition of $45 \mathrm{ml}$ Millipore $\mathrm{H}_{2} \mathrm{O}$

\section{B.6.2 $\mathrm{BeF}_{2}$ - Leaching (only when NO Al-chemistry will be done)}

$\mathrm{BeF}_{2}$ is water soluble while many other cations (including Al) are less so.

Add $15 \mathrm{ml}$ Millipore $\mathrm{H}_{2} \mathrm{O}$ to fluoride cake (to cover bottom of beaker)

Shake and heat gently for $\sim 20$ minutes on hotplate

Pipette off $15 \mathrm{ml}$ supernate (containing water-soluble $\mathrm{BeF}_{2}, \mathrm{TiF}_{4}, \mathrm{Fe}(\mathrm{II}) \mathrm{F}_{2}$ but no $\mathrm{AlF}_{3}$ ) into clean $22 \mathrm{ml}$ Savillex beakers (or $180 \mathrm{ml}$ beakers for dirty samples).

Repeat these three steps 2 more times (a total of $15 \mathrm{ml}$ liquid for clean samples of $45 \mathrm{ml}$ for dirty samples)

Heat the samples at $\sim 120^{\circ} \mathrm{C}$ to evaporate the solution (22ml Savillex beakers for clean or $180 \mathrm{ml}$ for dirty samples)

Add 10ml 6M HCl

Transfer solution into cleaned $15 \mathrm{ml}$ centrifuge tubes

Centrifuge for 5 minutes @ 3000 rpm 
Load only the supernate to the columns in the following step.

If available, check the purity of your samples at this point using ICP-OES

\section{B.7 Column Fe}

2ml Biorad AG1-X8 100-200 mesh (anion) resin in 15ml Eichrom columns stored in $\mathrm{H}_{2} \mathrm{O}$.

Note: to prepare new columns 1) place empty columns in stand with shot glasses below, 2) add tip to one column, add $2 \mathrm{ml}$ of $\mathrm{H}_{2} \mathrm{O}$ and mark line (then mark line on other columns), 3) add water and resin to a small beaker and agitate, 4) wet filter for each column and check they are dripping, 5) pipette resin into each column, letting it settle, and continue until resin reaches the marked line, 6) to store, fill with $\mathrm{H}_{2} \mathrm{O}$, cap tops and add tips to ends.

Open column and let the water drip out (drain to waste container)

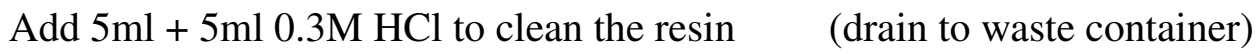

Add $2 \mathrm{ml}+2 \mathrm{ml}+2 \mathrm{ml} 6 \mathrm{M} \mathrm{HCl}$ to condition the resin (drain to waste container)

Add sample $(10 \mathrm{ml} 6 \mathrm{M} \mathrm{HCl}) \quad$ Collect Be $(+\mathbf{A l})$ in $22 \mathrm{ml}$ Savillex beakers

Add $2 \mathrm{ml}+2 \mathrm{ml}+2 \mathrm{ml} 6 \mathrm{M} \mathrm{HCl}$ to elute Be Collect Be $(+\mathbf{A l})$ in $22 \mathrm{ml}$ Savillex beakers

Add $5 \mathrm{ml}+5 \mathrm{ml} 0.3 \mathrm{M} \mathrm{HCl}$ to clean the resin (drain to waste container)

Add Millipore $\mathrm{H}_{2} \mathrm{O}$ and seal the columns for storage.

Heat the samples at $\sim 120^{\circ} \mathrm{C}$ to evaporation

Add $4 \mathrm{ml}$ (20ml for dirty quartz) $0.4 \mathrm{M}$ oxalic acid (binds $\mathrm{Al}$ and Ti for faster passing through resin) to each column

Note: to make up $0.4 \mathrm{M}$ oxalic acid, add $2 \mathrm{x} \sim 50.6 \mathrm{~g}$ oxalic powder (measured on tared filter paper) to cleaned $2 \mathrm{~L}$ bottle, then add $\sim 2 \mathrm{~L}$ Millipore $\mathrm{H}_{2} \mathrm{O}$, shake and leave to dissolve for $\sim 2$ hours.

Warm the samples at $60^{\circ} \mathrm{C}$ with lid for $\sim 2$ hours

\section{Note: the acid breaks if too warm}

Remove from hotplate and allow to cool down for at least 30 minutes

Transfer the samples to new $15 \mathrm{ml}$ centrifuge tubes 
Centrifuge for 5 minutes @ 3000 rpm

Load only the supernate to the columns in the following step

\section{B.8 Column Be}

\section{B.8.1 1ml Column Be (Clean Samples: Al < 5mg)}

$1 \mathrm{ml}$ Biorad AG50-X8 200-400 mesh (cation) resin in 7.5ml RKBN104704 columns stored in $\mathrm{H}_{2} \mathrm{O}$.

Note: check acid concentrations with density meter

$0.5 \mathrm{M} \mathrm{HNO}_{3}=$ density of $\sim 1.016$ (for $140 \mathrm{ml}, 135.4 \mathrm{ml} \mathrm{H}_{2} \mathrm{O}+\sim 4.7 \mathrm{ml}$ conc. $(15 \mathrm{M}) \mathrm{HNO}_{3}$ )

$1 \mathrm{M} \mathrm{HNO}_{3}=$ density of $\sim 1.033$ (for $150 \mathrm{ml}, 140 \mathrm{ml} \mathrm{H} \mathrm{H}_{2} \mathrm{O}+\sim 10 \mathrm{ml}$ conc. $(15 \mathrm{M}) \mathrm{HNO}_{3}$ )

Open column and let the water drip out (drain to waste container)

Add $2 \mathrm{ml}+3 \mathrm{ml} \sim 5 \mathrm{M} \mathrm{HNO}_{3}$ to clean the resin (drain to waste container)

Add $2 \mathrm{ml}+3 \mathrm{ml}$ Millipore $\mathrm{H}_{2} \mathrm{O}$ to remove $\mathrm{HNO}_{3}$ from the resin (drain to waste container)

Add $2 \mathrm{ml}+3 \mathrm{ml} 0.4 \mathrm{M}$ oxalic acid to condition resin (drain to waste container)

Add sample (4ml 0.4M oxalic acid) (drain to clean container)

Add $1 \mathrm{ml} 0.4 \mathrm{M}$ oxalic acid to wash the sample down (drain to clean container)

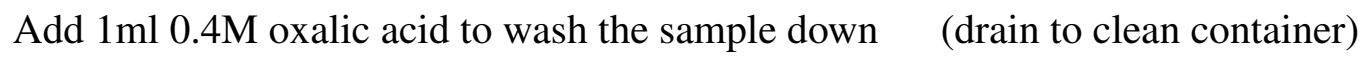

Add $10 \mathrm{ml} 0.4 \mathrm{M}$ oxalic acid: to elute $\mathrm{Fe}, \mathrm{Al}$, Ti etc. (drain to clean container)

Add $1 \mathrm{ml}+2 \mathrm{ml}$ Millipore $\mathrm{H}_{2} \mathrm{O}$ to remove oxalic acid (drain to clean container)

Note: sample in $\mathrm{H}_{2} \mathrm{O}$ and oxalic acid will need to be collected in 2x clean $11 \mathrm{ml}$ centrifuge tubes per column

Add $2 \mathrm{ml}+2 \mathrm{ml}+4 \mathrm{ml} 0.5 \mathrm{M} \mathrm{HNO}_{3}$ to elute $\mathrm{Na} \quad$ (drain to clean container)

Note: Na will need to be collected in $1 \mathrm{x}$ cleaned $11 \mathrm{ml}$ centrifuge tube per column

Add $3 \mathrm{ml}+3 \mathrm{ml}+5 \mathrm{ml} 1 \mathrm{M} \mathrm{HNO}_{3}$ to elute $\mathrm{Be} \quad$ Collect Be in new clean $15 \mathrm{ml}$ centrifuge tube

Add $10 \mathrm{ml} 5 \mathrm{M} \mathrm{HNO}_{3}$ to clean resin (drain to clean container) 
Note: post-Be wash will need to be collected in $1 \mathrm{x}$ cleaned $11 \mathrm{ml}$ centrifuge tube per column

Add $5 \mathrm{ml} \mathrm{H}_{2} \mathrm{O}$ remove $5 \mathrm{M} \mathrm{HNO}_{3} \quad$ (drain to waste container)

Add Millipore $\mathrm{H}_{2} \mathrm{O}$ and seal the columns for storage

\section{B.8.2 5ml Column Be (Dirty Samples: Al > 5mg)}

5ml Biorad AG50-X8 200-400 mesh (cation) resin in $15 \mathrm{ml}$ Eichrom columns stored in $\mathrm{H}_{2} \mathrm{O}$.

Open column and let the water drip out (drain to waste container)

Add $5 \mathrm{ml}+10 \mathrm{ml} \sim 5 \mathrm{M} \mathrm{HNO}_{3}$ to clean the resin (drain to waste container)

Add $5 \mathrm{ml}+5 \mathrm{ml}$ Millipore $\mathrm{H}_{2} \mathrm{O}$ to remove $\mathrm{HNO}_{3}$ from the resin (drain to waste container)

Add $5 \mathrm{ml}+10 \mathrm{ml} 0.4 \mathrm{M}$ oxalic acid to condition resin (drain to waste container)

Add sample (20ml 0.4M oxalic acid) (drain to clean container)

Add $5 \mathrm{ml} \mathrm{0.4M} \mathrm{oxalic} \mathrm{acid} \mathrm{to} \mathrm{wash} \mathrm{the} \mathrm{sample} \mathrm{down} \mathrm{(drain} \mathrm{to} \mathrm{clean} \mathrm{container)}$

Add $5 \mathrm{ml} \mathrm{0.4M} \mathrm{oxalic} \mathrm{acid} \mathrm{to} \mathrm{wash} \mathrm{the} \mathrm{sample} \mathrm{down} \mathrm{(drain} \mathrm{to} \mathrm{clean} \mathrm{container)}$

Add 50ml 0.4M oxalic acid: to elute Fe, Al, Ti etc. (drain to clean container)

Add $5 \mathrm{ml}+10 \mathrm{ml}$ Millipore $\mathrm{H}_{2} \mathrm{O}$ to remove oxalic acid (drain to clean container)

Note: sample in $\mathrm{H}_{2} \mathrm{O}$ and oxalic acid will need to be collected in $2 \mathrm{x}$ clean $50 \mathrm{ml}$ centrifuge tubes per column

Add $15 \mathrm{ml}+25 \mathrm{ml} 0.5 \mathrm{M} \mathrm{HNO}_{3}$ to elute $\mathrm{Na} \quad$ (drain to clean container)

Note: Na will need to be collected in $1 \mathrm{x}$ clean $50 \mathrm{ml}$ centrifuge tube per column

Add $20 \mathrm{ml} 1 \mathrm{M} \mathrm{HNO}_{3}$ wash (drain to clean container)

Note: post-Na wash will need to be collected in $1 \times 50 \mathrm{ml}$ (or $2 \mathrm{x} 11 \mathrm{ml}$ ) clean centrifuge tubes per column

Add $20 \mathrm{ml}+20 \mathrm{ml} 1 \mathrm{M} \mathrm{HNO}_{3}$ to elute $\mathrm{Be} \quad$ Collect Be in new clean 90ml Savillex beakers

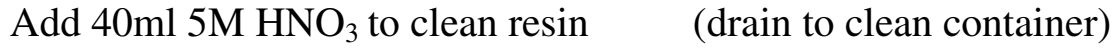


Note: post-Be wash will need to be collected in $1 \mathrm{x}$ clean $50 \mathrm{ml}$ centrifuge tubes per column

Add $5 \mathrm{ml}+15 \mathrm{ml} \mathrm{H}_{2} \mathrm{O}$ remove $5 \mathrm{M} \mathrm{HNO}_{3}$ (drain to waste container)

Add Millipore $\mathrm{H}_{2} \mathrm{O}$ and seal the columns for storage

Heat the samples at $\sim 120^{\circ} \mathrm{C}$ to evaporate the solution

Add $11 \mathrm{ml} 1 \mathrm{M} \mathrm{HNO}_{3}$ to dissolve the sample and transfer to a new $15 \mathrm{ml}$ centrifuge tube

\section{B.9 Column Al}

1ml Biorad AG1-X8 100-200 mesh (anion) resin in 7.5ml columns stored in $\mathrm{H}_{2} \mathrm{O}$

Note: to prepare mixture of $0.05 \mathrm{M}$ Oxalic Acid and $0.5 \mathrm{M} \mathrm{HCl}$, mix equal amounts of $0.1 \mathrm{M}$ oxalic Acid and $1 \mathrm{M} \mathrm{HCl}$.

Open column and let $\mathrm{H}_{2} \mathrm{O}$ drop out (drain to waste container)

Add $2 \mathrm{ml}$ Millipore $\mathrm{H}_{2} \mathrm{O}$ to clean resin (drain to waste container)

Add $5 \mathrm{ml}+5 \mathrm{ml} 0.3 \mathrm{M} \mathrm{HCl}$ to clean resin (drain to waste container)

Add $2 \mathrm{ml}+2 \mathrm{ml}$ Millipore $\mathrm{H}_{2} \mathrm{O}$ to remove $\mathrm{HCl}$ (drain to waste container)

Add $2 \mathrm{ml}+2 \mathrm{ml}+2 \mathrm{ml} 0.4 \mathrm{M}$ oxalic Acid to condition resin (drain to waste container)

Add sample ( $0.4 \mathrm{M}$ oxalic acid from Be-column) (drain to clean container)

Add $4 \mathrm{ml}+2 \mathrm{ml} 0.05 \mathrm{M} \mathrm{Ox} / 0.5 \mathrm{M} \mathrm{HCl}$ wash (drain to clean container)

Note: pre-Al effluent will need to be collected in 1x cleaned $50 \mathrm{ml}$ centrifuge tube per column (in case Al elutes early)

Add $5 \mathrm{ml}+5 \mathrm{ml} 0.05 \mathrm{M} \mathrm{Ox} / 0.5 \mathrm{M} \mathrm{HCl}$ Collect $\mathbf{A l}$ in new clean $22 \mathrm{ml}$ Savillex beakers

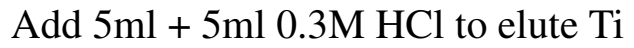

Note: Ti will need to be collected in $1 \mathrm{x}$ cleaned $11 \mathrm{ml}$ centrifuge tube per column

Note: to make up $0.3 \mathrm{M} \mathrm{HCl}$ (for $140 \mathrm{ml}), 136 \mathrm{ml} \mathrm{H}_{2} \mathrm{O}+4 \mathrm{ml}(\sim 10.5 \mathrm{M}) \mathrm{HCl}$.

Seal and store column in Millipore $\mathrm{H}_{2} \mathrm{O}$ 
Dry down sample in Savillex beaker on hotplate (over 1/2 day or overnight)

Add $2 \mathrm{ml}$ Aqua Regia $\left(15 \mathrm{M} \mathrm{HNO}_{3} 1: 16 \mathrm{M} \mathrm{HCl}\right)$ and leave on hotplate to dissolve with lid Note: to make up $\sim 30 \mathrm{ml}$, add $15 \mathrm{ml} \mathrm{HNO}_{3}(15 \mathrm{M})+\sim 8.6 \mathrm{ml} \mathrm{HCl}(\sim 10.5 \mathrm{M})+6.4 \mathrm{ml}$ $\mathrm{H}_{2} \mathrm{O}$

Remove lid and evaporate on hotplate to dryness

Repeat once

Add $1 \mathrm{ml} 15 \mathrm{M} \mathrm{HNO}_{3}$ and $1 \mathrm{ml} \mathrm{H}_{2} \mathrm{O}_{2}$

Evaporate on hotplate to dryness

Repeat $2 \mathrm{x}$

Go to Al precipitation or silver addition

\section{B.10 Precipitation}

\section{B.10.1 Be precipitation}

Add $\sim 1-1.5 \mathrm{ml}$ concentrated $(\sim 25 \%) \mathrm{NH}_{4} \mathrm{OH}$ to each sample (will be $\mathrm{pH} \approx 10$ )

Shake the sample well until a $\mathrm{BeOH}$ precipitate forms

Note: small white specks will appear in the solution

Centrifuge for 5 minutes @ 3000 rpm

Note: you should see a small milky gel at the bottom of the tube, this is $\mathrm{BeOH}$ !

Decant the supernate into the $5 \mathrm{M} \mathrm{HNO}_{3}$ centrifuge tubes with post-Be wash

Redissolve the precipitate in $5 \mathrm{ml} 1 \mathrm{M} \mathrm{HNO}_{3}$

Transfer to new $11 \mathrm{ml}$ or $15 \mathrm{ml}$ centrifuge tubes

Add $\sim 0.5 \mathrm{ml}$ concentrated $\mathrm{NH}_{4} \mathrm{OH}$ to each sample

Centrifuge for 5 minutes @ 3000 rpm

Decant the supernate

Add $3 \mathrm{ml} \mathrm{H}_{2} \mathrm{O}$ and shake well to dissociate the precipitate 
Centrifuge for 5 minutes @ 3000 rpm

Decant the supernate

\section{B.10.2 Al precipitation}

Add $1 \mathrm{ml} 1 \mathrm{M} \mathrm{HNO}_{3}$ to samples (in Savillex beaker) and dissolve on hotplate

Transfer samples to cleaned $1.5 \mathrm{ml}$ centrifuge tube

Add $\sim 200 \mu \mathrm{L}$ conc. $\mathrm{NH}_{4} \mathrm{OH}$ to each sample

Shake the sample well until a precipitate forms

Note: white specks will appear in the solution (more obvious than with $\mathrm{Be}$ )

Centrifuge for 5 minutes @ 3000 rpm

Decant the supernate

Add $\mathrm{H}_{2} \mathrm{O}$ and shake well to dissociate the precipitate

Centrifuge for 5 minutes @ 3000 rpm

Decant the supernate

Add $\mathrm{H}_{2} \mathrm{O}$ and shake well to dissociate the precipitate

Centrifuge for 5 minutes @ 3000 rpm 


\section{Appendix C}

\section{Analysis of surface-exposure data}

Included here are the MATLAB scripts developed to model complex burial-exposure histories of samples at Skelton Glacier (Chapter 5) and to estimate the rate and duration of rapid thinning at Mackay Glacier (Chapter 7). The latter was achieved with assistance of E. Smith.

\% precedes explanatory comments.

\section{C.1 Complex burial-exposure history model}

The below code uses ice sheet model output from Pollard and DeConto (2009), which states whether the mouth of Skelton Glacier was occupied by grounded ice or open water/ice-shelf ('PD_modelled_ice_Skelton.mat '), provided at 1-ka intervals since $5 \mathrm{Ma}$ before present. Orbital cycles (eccentricity and obliquity) used for 15-5 Ma are sourced from AnalySeries 2.0 (Paillard et al., 1996).

See Chapter 5 for explanation of parameters used to calculate evolving nuclide concentrations and force burial and exposure through time.

\section{Skelton_complexhistory.m}

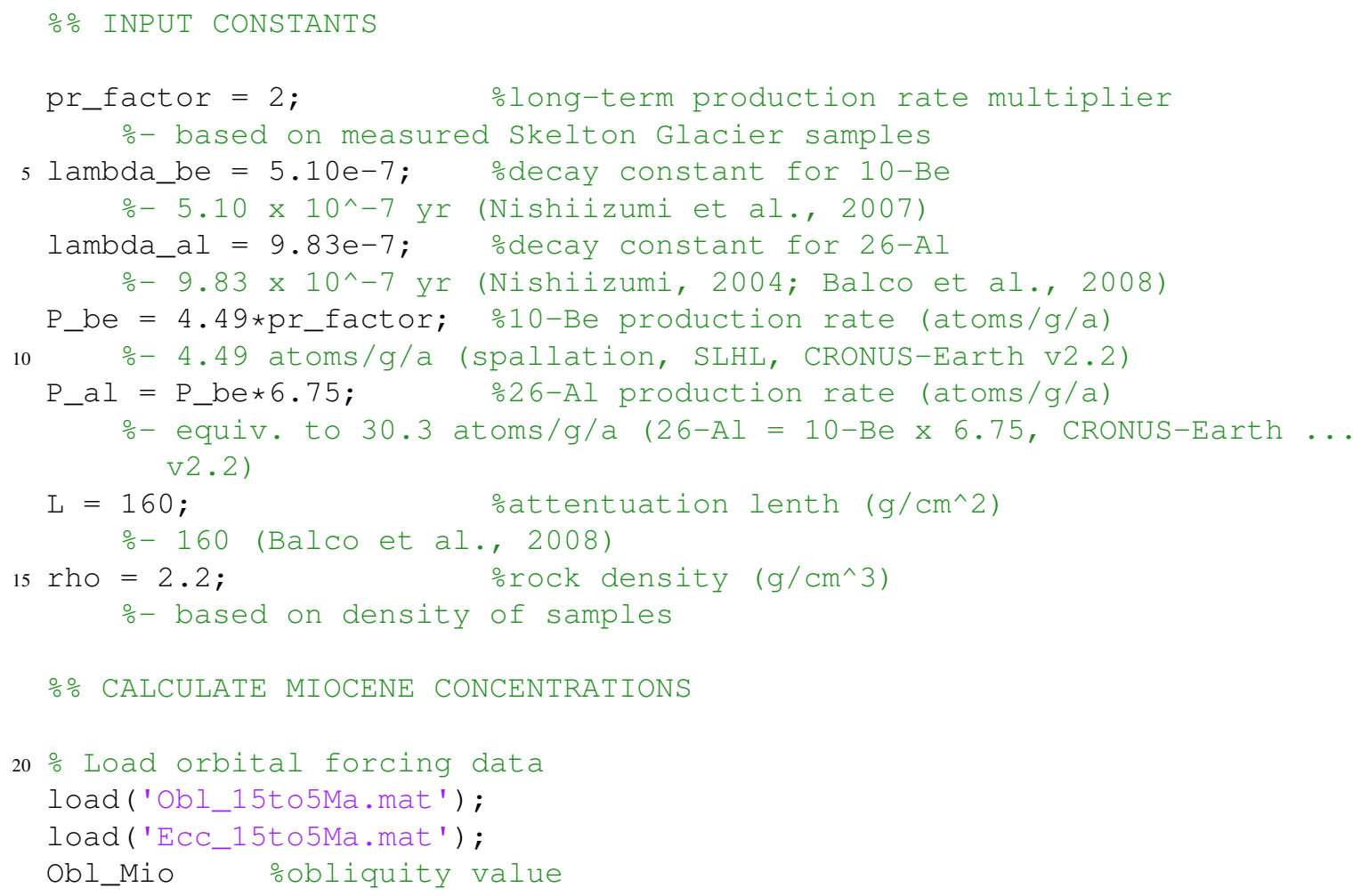




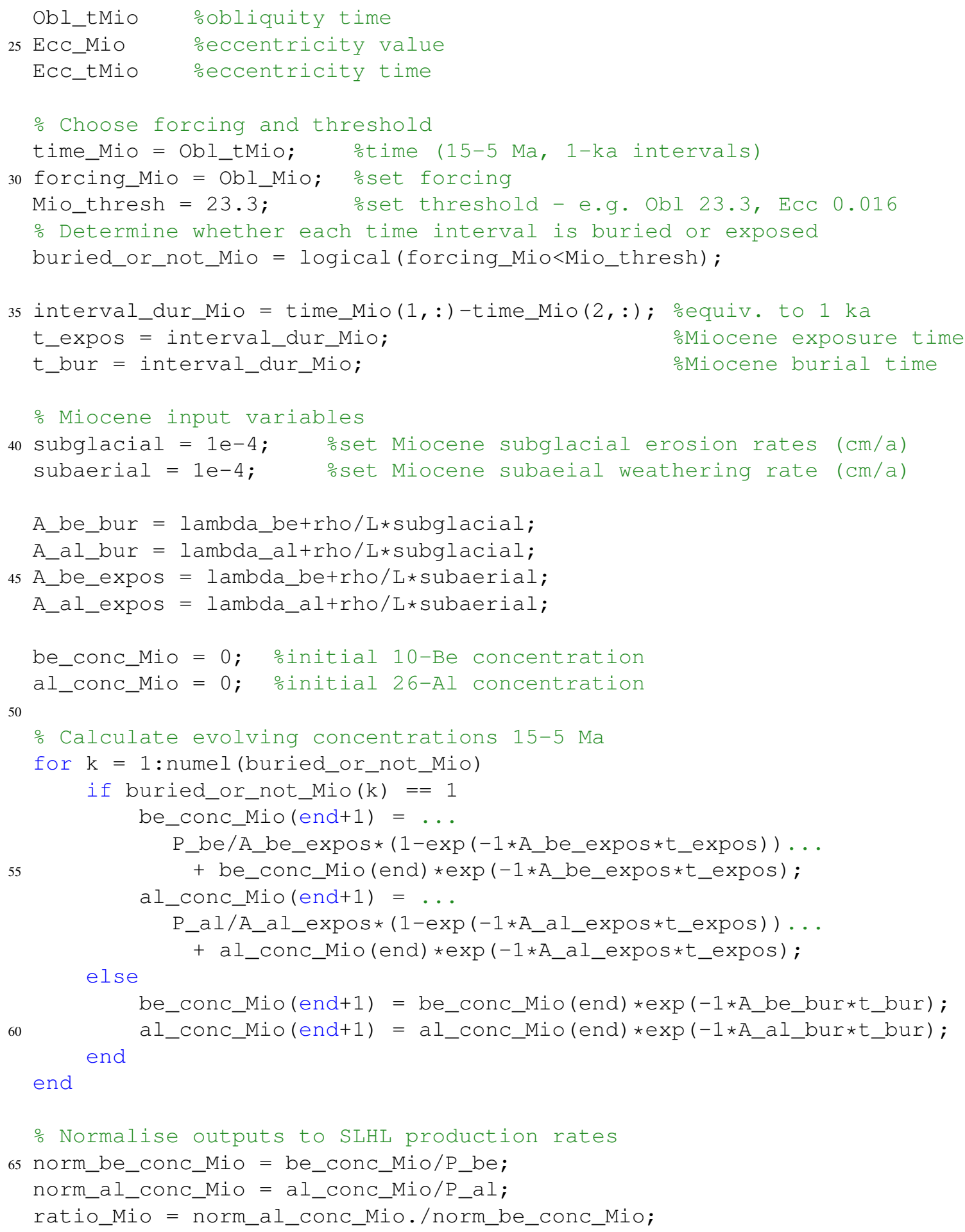

$70 \%$ CALCULATE PLIOCENE-TO-PRESENT CONCENTRATIONS

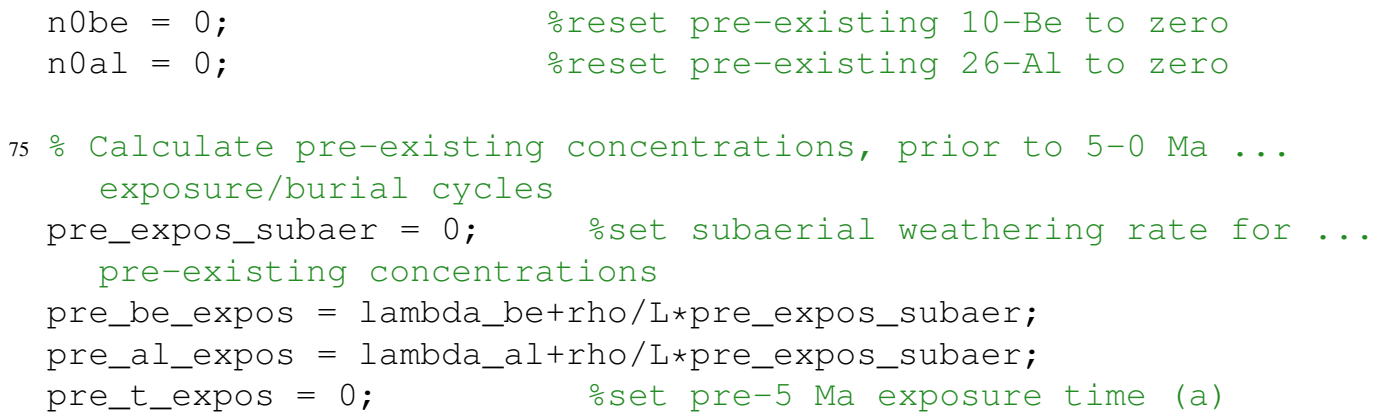




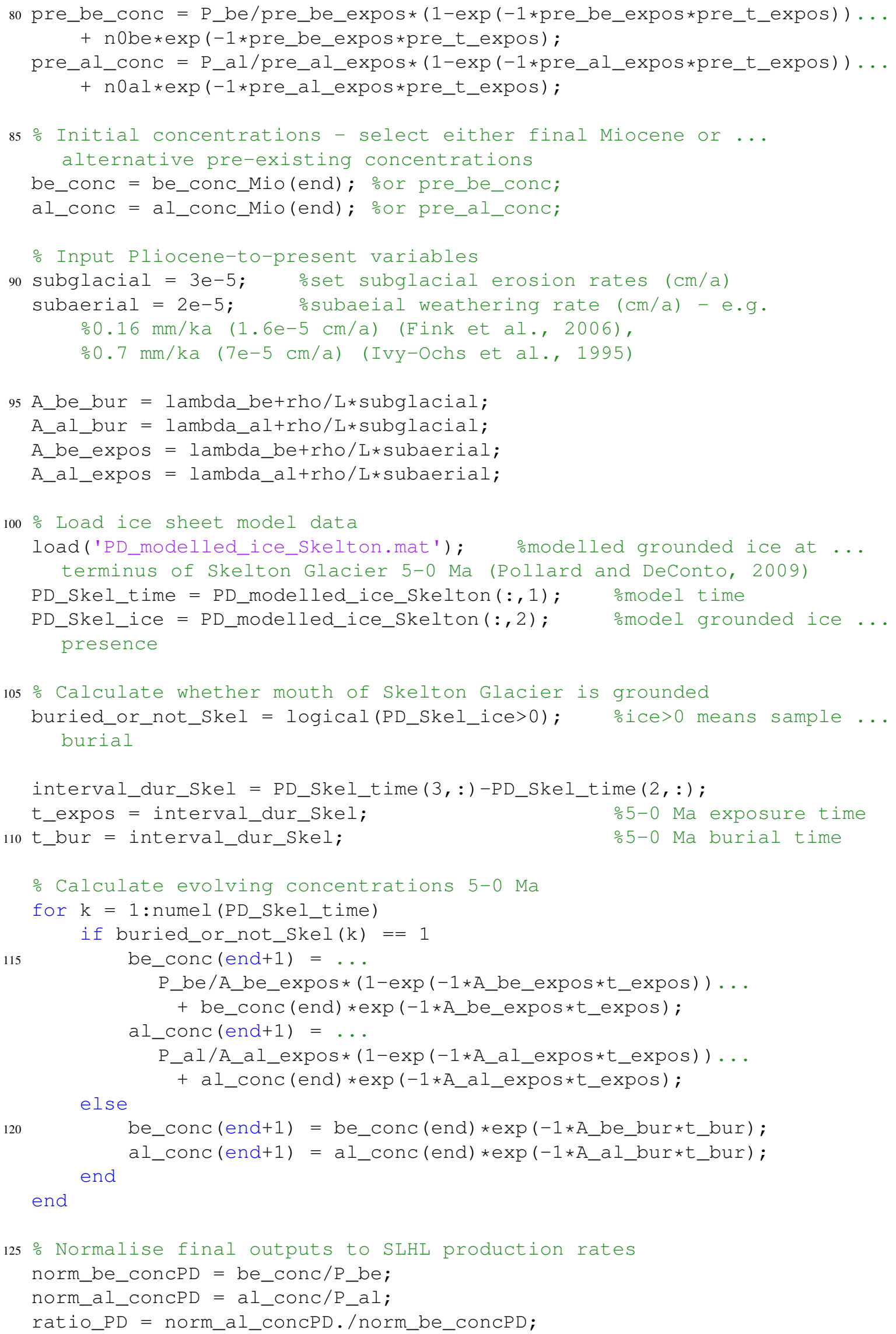




\section{C.2 Regression of rapid thinning through Monte Carlo simulations}

The below code requires an input file of recorded surface-exposure ages from a "dipstick" transect (e.g. 'GR_upper_sampleages.txt'), including sample elevation, mean age and $1 \sigma$ uncertainty. Least-squares regression analysis uses both weighted (using sample age uncertainties) and unweighted methods. The number of Monte Carlo simulations performed on the data can be chosen. Negative-sloping regressions are not feasible and are therefore removed. This script also plots the modelled thinning rate probabilities and regression profiles with uncertainties. Durations of the recorded rapid thinning were also calculated in Chapter 7, using maximum and minimum regression values for each transect.

\section{Thinning_regression.m}

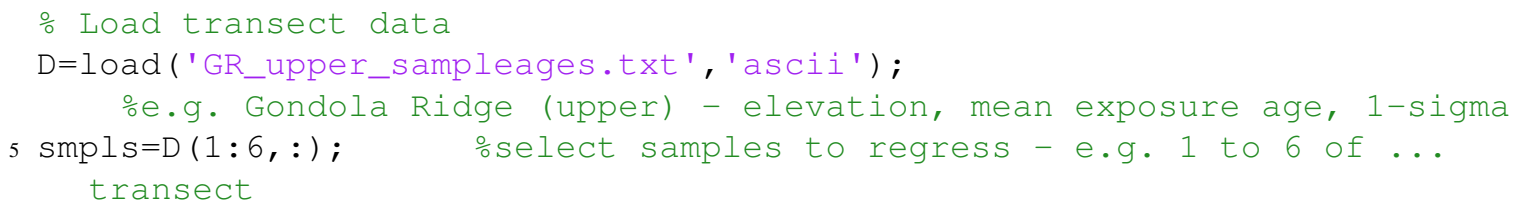




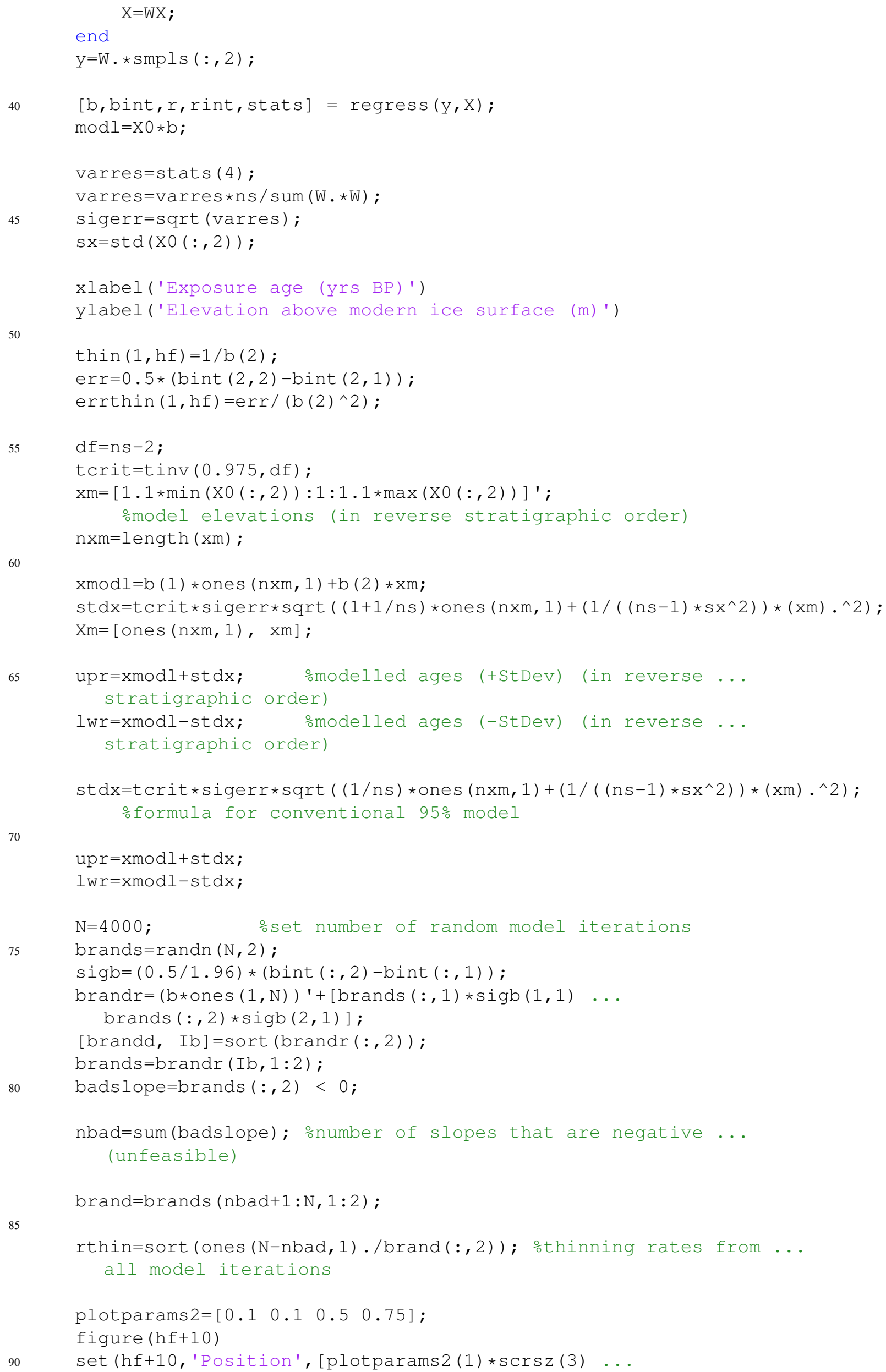


plotparams2 (2)*scrsz (4) plotparams2 (3)*scrsz (3) ...

plotparams2 (4) *scrsz (4) ]);

$\operatorname{semilogx}\left(0,0,{ }^{\prime} \cdot \mathrm{k}^{\prime}\right)$

hold on

$\operatorname{stairs}\left(\right.$ rthin, $\left.(1 /(\mathrm{N}-\mathrm{nbad})) *[1: \mathrm{N}-\mathrm{nbad}], \mathrm{I}^{\prime}-\mathrm{k}^{\prime}\right)$

hold on

plot ([min(rthin) $\max (r t h i n)],[0.9750 .975]$, ' $^{\prime}$ ' $^{\prime}$ )

plot ([min(rthin) $\left.\max (r t h i n)],[0.0250 .025],{ }^{\prime}-r^{\prime}\right)$

plot ([min(rthin) $\max (r t h i n)],[0.8420 .842]$, '--r') $^{\prime}$

plot ([min(rthin) $\left.\max (r t h i n)],[0.1590 .159],{ }^{\prime}--r^{\prime}\right)$

xlabel ('Thinning rate $\left(\operatorname{m} \operatorname{yr}^{\wedge}\{-1\}\right)$ ')

ylabel ('Cumulative probability')

title('Distribution of (non-negative) random thinning rates')

low95up (hf, 1:2) = [quantile (rthin,.025,1); quantile (rthin,.975,1) ] ; olower \& upper 95\% modelled thinning rates (m/yr)

low68up (hf, 1:2) = [quantile (rthin,.159,1); quantile(rthin,.842,1)] ; olower \& upper 68\% modelled thinning rates (m/yr)

randmod $=\mathrm{Xm} *$ brand ' ;

ages from all regression ...

iterations

qu=quantile $($ randmod, $.975,2) ; \quad$ oupper quantile of modelled ... ages (in reverse stratigraphic order)

ql=quantile $($ randmod, $.025,2) ; \quad$ olower quantile of modelled ... ages (in reverse stratigraphic order)

grey $=\left[\begin{array}{lll}0.85 & 0.85 & 0.85\end{array}\right]$;

$\mathrm{xm}=\mathrm{xm}+\mathrm{midx} *$ ones $(\mathrm{nxm}, 1)$;

$\mathrm{dxm}=\mathrm{x} 0(:, 2)+\operatorname{midx} *$ ones $(\mathrm{ns}, 1)$;

figure (hf)

if $h f==1$

plot (modl, dxm, '-m', 'LineWidth' , 2)

plot (randmod $(:, 1), x m, '-1$, 'color', grey)

plot (qu, xm, ': ', 'color', 'm' ,' 'Linewidth', 1.5)

plot (ql, xm, ': ', 'color', 'm', 'LineWidth', 1.5)

legend('Model median', 'Random models', 'Upper 97.5\% ...

quantile', 'Lower 2.5\% quantile', 'Location', 'Northwest')

for $j=1: N-n b a d$

end

plot (randmod $(:, j), x m, '-'$, 'color', grey)

errorbar_x (smpls $(:, 2), d x m, 2 * \operatorname{smpls}(:, 3),{ }^{\prime}$ ok' $)$

plot (modl, dxm, '-m', 'LineWidth', 2)

plot (qu, xm, ': ', 'color', 'm', 'Linewidth', 1.5)

plot (ql, xm, ': ', 'color', 'm', 'Linewidth', 1.5)

else

plot (modl, dxm, '-b' ', 'LineWidth', 2)

plot (randmod $(:, 1), x m, '-1$, 'color', grey)

plot (qu, xm, ': ', 'color', 'b' ', 'Linewidth', 1.5)

plot (ql, xm, ': ', 'color', 'b' ', 'Linewidth', 1.5)

legend('Model median', 'Random models', 'Upper 97.5\%...

quantile', 'Lower 2.5\% quantile', 'Location', 'SouthEast' )

for $j=1: N-n b a d$

end

plot (randmod $(:, j), x m,{ }^{-}$', 'color', grey)

errorbar_x (smpls $\left.(:, 2), \mathrm{dxm}, 2 \star \operatorname{smpls}(:, 3), \mathrm{s}^{\prime} \mathrm{ok}\right)$

plot (modl, dxm, '-b' ', 'LineWidth' , 2)

plot (qu, xm, ': ', 'color', 'b' ', 'LineWidth', 1.5)

plot (ql, xm, ': ', 'color', 'b' ', 'LineWidth', 1.5) 
end

145

hold on

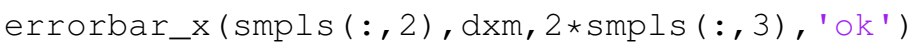
box on

end 



\section{References}

Ackert, R. (2008). Swinging gate or Saloon doors: Do we need a new model of Ross Sea deglaciation? WAIS Conference abstract.

Ackert, R. P., Barclay, D. J., Borns, H. W., Calkin, P. E., Kurz, M. D., Fastook, J. L., and Steig, E. J. (1999). Measurements of Past Ice Sheet Elevations in Interior West Antarctica. Science, 286:276-279.

Ackert, R. P., Mukhopadhyay, S., Parizek, B. R., and Borns, H. W. (2007). Ice elevation near the West Antarctic Ice Sheet divide during the Last Glaciation. Geophysical Research Letters, 342:L21506.

Alley, R., Blankenship, D., Bentley, C., and Rooney, S. (1986). Deformation of till beneath ice stream B, West Antarctica. Nature.

Anandakrishnan, S., Alley, R., Jacobel, R., and Conway, H. (2001). The flow regime of Ice Stream $\mathrm{C}$ and hypotheses concerning its recent stagnation. The West Antarctic Ice Sheet: Behavior and Environment, pages 283-296.

Anderson, B. M., Hindmarsh, R. C. A., and Lawson, W. J. (2004). A modelling study of the response of Hatherton Glacier to Ross Ice Sheet grounding line retreat. Global and Planetary Change, 42:143-153.

Anderson, J. B. and Andrews, J. T. (1999). Radiocarbon constraints on ice sheet advance and retreat in the Weddell Sea, Antarctica. Geology, 27(2):179-182.

Anderson, J. B., Conway, H., Bart, P. J., Witus, A. E., Greenwood, S. L., McKay, R. M., Hall, B. L., Ackert, R. P., Licht, K., and Jakobsson, M. (2014). Ross Sea paleo-ice sheet drainage and deglacial history during and since the LGM. Quaternary Science Reviews.

Anderson, J. B., Shipp, S. S., Lowe, A. L., Wellner, J. S., and Mosola, A. B. (2002). The Antarctic Ice Sheet during the Last Glacial Maximum and its subsequent retreat history: a review. Quaternary Science Reviews, 21(1):49-70.

Argento, D. C., Reedy, R. C., and Stone, J. O. (2013). Modeling the earth's cosmic radiation. Nuclear Instruments and Methods in Physics Research Section B: Beam Interactions with Materials and Atoms, 294:464-469.

Atkins, C. (2013). Geomorphological evidence of cold-based glacier activity in South Victoria Land, Antarctica. Geological Society, London, Special Publications, 381(1):299-318.

Atkins, C., Barrett, P., and Hicock, S. (2002). Cold glaciers erode and deposit: Evidence from Allan Hills, Antarctica. Geology, 30(7):659-662.

Augustinus, P. C., Gore, D. B., Leishman, M. R., Zwartz, D., and Colhoun, E. A. (1997). Reconstruction of ice flow across the Bunger Hills, East Antarctica. Antarctic Science, 9(03):347-354.

Balco, G. (2011). Contributions and unrealized potential contributions of cosmogenicnuclide exposure dating to glacier chronology, 1990-2010. Quaternary Science Reviews, 30(1-2):3-27. 
Balco, G. and Schaefer, J. M. (2013). Exposure-age record of Holocene ice sheet and ice shelf change in the northeast Antarctic Peninsula. Quaternary Science Reviews, 59:101-111.

Balco, G., Stone, J. O., Lifton, N. A., and Dunai, T. J. (2008). A complete and easily accessible means of calculating surface exposure ages or erosion rates from ${ }^{10} \mathrm{Be}$ and ${ }^{26} \mathrm{Al}$ measurements. Quaternary Geochronology, 3(3):174-195.

Balco, G., Stone, J. O., Sliwinski, M. G., and Todd, C. (2014). Features of the glacial history of the Transantarctic Mountains inferred from cosmogenic ${ }^{26} \mathrm{Al},{ }^{10} \mathrm{Be}$ and ${ }^{21} \mathrm{Ne}$ concentrations in bedrock surfaces. Antarctic Science, 26(06):708-723.

Bard, E., Hamelin, B., Arnold, M., Montaggioni, L., Cabioch, G., Faure, G., and Rougerie, F. (1996). Deglacial sea-level record from Tahiti corals and the timing of global meltwater discharge. Nature, 382(6588):241-244.

Bard, E., Hamelin, B., and Delanghe-Sabatier, D. (2010). Deglacial Meltwater Pulse 1B and Younger Dryas Sea Levels Revisited with Boreholes at Tahiti. Science, 327:12351237.

Baroni, C. and Hall, B. L. (2004). A new Holocene relative sea-level curve for Terra Nova Bay, Victoria Land, Antarctica. Journal of Quaternary Science, 19(4):377-396.

Barrett, P., Adams, C., Mclntosh, W., Swisher, C., and Wilson, G. (1992). Geochronological evidence supporting Antarctic deglaciation three million years ago. Nature, 359(6398):816-818.

Barrett, P., Elston, D., Harwood, D., McKelvey, B., and Webb, P.-N. (1987). MidCenozoic record of glaciation and sea-level change on the margin of the Victoria Land basin, Antarctica. Geology, 15(7):634-637.

Bart, P., Anderson, J., Trincardi, F., and Shipp, S. (2000). Seismic data from the Northern basin, Ross Sea, record extreme expansions of the East Antarctic Ice Sheet during the late Neogene. Marine Geology, 166(1):31-50.

Bart, P. J. and Cone, A. N. (2012). Early stall of West Antarctic Ice Sheet advance on the eastern Ross Sea middle shelf followed by retreat at 27,500 ${ }^{14}$ CyrBP. Palaeogeography, Palaeoclimatology, Palaeoecology, 335:52-60.

Bartek, L. R., Andersen, J. L., and Oneacre, T. A. (1997). Substrate control on distribution of subglacial and glaciomarine seismic facies based on stochastic models of glacial seismic facies deposition on the Ross Sea continental margin, Antarctica. Marine Geology, 143(1):223-262.

Benn, D. I., Hulton, N. R., and Mottram, R. H. (2007). 'Calving laws', 'sliding laws' and the stability of tidewater glaciers. Annals of Glaciology, 46(1):123-130.

Bennett, K. (1992). PSIMPOLL: a quickBASIC program that generates PostScript page description files of pollen diagrams. INQUA Commission for the study of the Holocene: working group on data handling methods newsletter, 8:11-12.

Bentley, M. J., Fogwill, C. J., Kubik, P. W., and Sugden, D. E. (2006). Geomorphological evidence and cosmogenic ${ }^{10} \mathrm{Be} /{ }^{26} \mathrm{Al}$ exposure ages for the Last Glacial Maximum and deglaciation of the Antarctic Peninsula Ice Sheet. Bulletin of the Geological Society of America, 118(9-10):1149-1159. 
Bentley, M. J., Fogwill, C. J., Le Brocq, A. M., Hubbard, A. L., Sugden, D. E., Dunai, T. J., and Freeman, S. P. (2010). Deglacial history of the West Antarctic Ice Sheet in the Weddell Sea embayment: Constraints on past ice volume change. Geology, 38(5):411414.

Bentley, M. J., O’Cofaigh, C., Anderson, J. B., Conway, H., Davies, B., Graham, A. G., Hillenbrand, C.-D., Hodgson, D. A., Jamieson, S. S., Larter, R. D., et al. (2014). A community-based geological reconstruction of Antarctic Ice Sheet deglaciation since the Last Glacial Maximum. Quaternary Science Reviews, 100:1-9.

Berg, S., Wagner, B., Cremer, H., Leng, M. J., and Melles, M. (2010). Late Quaternary environmental and climate history of Rauer Group, East Antarctica. Palaeogeography, Palaeoclimatology, Palaeoecology, 297(1):201-213.

Bindschadler, R. and Choi, H. (2007). Increased water storage at ice-stream onsets: a critical mechanism? Journal of Glaciology, 53(181):163-171.

Bindschadler, R., Nowicki, S., Abe-Ouchi, A., Aschwanden, A., Choi, H., Fastook, J., Granzow, G., Greve, R., Gutowski, G., Herzfeld, U., Jackson, C., Johnson, J., Khroulev, C., Levermann, A., Lipscomb, W., Martin, M., Morlighem, M., Parizek, B., Pollard, D., Price, S., Ren, D., Saito, F., Sato, T., Seddik, H., Seroussi, H., Takahashi, K., Walker, R., and Wang, W. (2013). Ice-sheet model sensitivities to environmental forcing and their use in projecting future sea level (The SeaRISE Project). Journal of Glaciology, 59:195-224.

Blaauw, M., Christen, J. A., et al. (2011). Flexible paleoclimate age-depth models using an autoregressive gamma process. Bayesian Analysis, 6(3):457-474.

Blanchon, P., Eisenhauer, A., Fietzke, J., and Liebetrau, V. (2009). Rapid sea-level rise and reef back-stepping at the close of the last interglacial highstand. Nature, 458(7240):881-884.

Bo, S., Siegert, M. J., Mudd, S. M., Sugden, D., Fujita, S., Xiangbin, C., Yunyun, J., Xueyuan, T., and Yuansheng, L. (2009). The Gamburtsev mountains and the origin and early evolution of the Antarctic Ice Sheet. Nature, 459(7247):690-693.

Bockheim, J. (2002). Landform and soil development in the McMurdo Dry Valleys, Antarctica: a regional synthesis. Arctic, Antarctic, and Alpine Research, pages 308317.

Bockheim, J. G., Wilson, S. C., Denton, G. H., Andersen, B. G., and Stuiver, M. (1989). Late Quaternary ice-surface fluctuations of Hatherton Glacier, Transantarctic Mountains. Quaternary Research, 31(2):229-254.

Boulton, G. (1974). Processes and patterns of glacial erosion. In Coates, D., editor, Glacial geomorphology. Proceedings of the Fifth Annual Geomorphology Symposia, Binghampton, pages 41-87. Allen \& Unwin, London.

Bradley, S., Siddall, M., Milne, G., Masson-Delmotte, V., and Wolff, E. (2013). Combining ice core records and ice sheet models to explore the evolution of the East Antarctic Ice sheet during the Last Interglacial period. Global and Planetary Change, 100:278290. 
Briggs, R., Pollard, D., and Tarasov, L. (2013). A glacial systems model configured for large ensemble analysis of Antarctic deglaciation. The Cryosphere Discussions, 7(2):1533-1589.

Briggs, R. D. and Tarasov, L. (2013). How to evaluate model-derived deglaciation chronologies: a case study using Antarctica. Quaternary Science Reviews, 63:109_ 127.

Briner, J. P., Miller, G. H., Davis, P. T., and Finkel, R. C. (2006). Cosmogenic radionuclides from fiord landscapes support differential erosion by overriding ice sheets. Geological Society of America Bulletin, 118(3-4):406-420.

Bromley, G., Hall, B., Stone, J., Conway, H., and Todd, C. (2010). Late Cenozoic deposits at Reedy Glacier, Transantarctic Mountains: implications for former thickness of the West Antarctic Ice Sheet. Quaternary Science Reviews, 29(3-4):384-398.

Bromley, G. R. M., Hall, B. L., Stone, J. O., and Conway, H. (2012). Late Pleistocene evolution of Scott Glacier, southern Transantarctic Mountains: implications for the Antarctic contribution to deglacial sea level. Quaternary Science Reviews, 50:1-13.

Brook, E. J., White, J. W., Schilla, A. S., Bender, M. L., Barnett, B., Severinghaus, J. P., Taylor, K. C., Alley, R. B., and Steig, E. J. (2005). Timing of millennial-scale climate change at Siple Dome, West Antarctica, during the last glacial period. Quaternary Science Reviews, 24(12):1333-1343.

Calkin, P. E. (1974). Subglacial geomorphology surrounding the ice-free valleys of Southern Victoria Land, Antarctica. Journal of Glaciology, 13:415-429.

Cameron, R. and Goldthwait, R. P. (1961). The US-IGY contribution to Antarctic glaciology. Ohio State University, Institute of Polar Studies.

Campbell, I., Jacobel, R., Welch, B., and Pettersson, R. (2008). The evolution of surface flow stripes and stratigraphic folds within Kamb Ice Stream: why don't they match? Journal of Glaciology, 54(186):421-427.

Carlson, A. E. and Clark, P. U. (2012). Ice sheet sources of sea level rise and freshwater discharge during the last deglaciation. Reviews of Geophysics, 50(4).

Chen, J. L., Wilson, C. R., Blankenship, D., and Tapley, B. D. (2009). Accelerated Antarctic ice loss from satellite gravity measurements. Nature Geoscience, 2(12):859-862.

Christl, M., Vockenhuber, C., Kubik, P., Wacker, L., Lachner, J., Alfimov, V., and Synal, H.-A. (2013). The ETH Zurich AMS facilities: Performance parameters and reference materials. Nuclear Instruments and Methods in Physics Research Section B: Beam Interactions with Materials and Atoms, 294:29-38.

Church, J., Clark, P., Cazenave, A., Gregory, J., Jevrejeva, S., Levermann, A., Merrifield, M., Milne, G., Nerem, R., Nunn, P., et al. (2013). Sea level change. In Climate Change 2013: The Physical Science Basis. Contribution of Working Group I to the Fifth Assessment Report of the Intergovernmental Panel on Climate Change, pages 1137-1216. Cambridge University Press, Cambridge, United Kingdom.

Clark, P. U. (2011). Deglacial history of the West Antarctic Ice Sheet in the Weddell Sea embayment: Constraints on past ice volume change: COMMENT. Geology, 39(5):e239-e239. 
Clark, P. U., Dyke, A. S., Shakun, J. D., Carlson, A. E., Clark, J., Wohlfarth, B., Mitrovica, J. X., Hostetler, S. W., and McCabe, A. M. (2009). The Last Glacial Maximum. Science, 325:710.

Clark, P. U., McCabe, A. M., Mix, A. C., and Weaver, A. J. (2004). Rapid rise of sea level 19,000 years ago and its global implications. Science, 304(5674):1141-1144.

Clark, P. U., Mitrovica, J. X., Milne, G. A., and Tamisiea, M. E. (2002). Sea-level fingerprinting as a direct test for the source of global meltwater pulse IA. Science, 295(5564):2438-2441.

Conway, H., Catania, G., Raymond, C., Gades, A., Scambos, T., and Engelhardt, H. (2002). Switch of flow direction in an Antarctic ice stream. Nature, 419(6906):465467.

Conway, H., Hall, B. L., Denton, G. H., Gades, A. M., and Waddington, E. D. (1999). Past and future grounding-line retreat of the West Antarctic Ice Sheet. Science, 286(5438):280-283.

Cook, C. P., van de Flierdt, T., Williams, T., Hemming, S. R., Iwai, M., Kobayashi, M., Jimenez-Espejo, F. J., Escutia, C., González, J. J., Khim, B.-K., et al. (2013). Dynamic behaviour of the East Antarctic ice sheet during Pliocene warmth. Nature Geoscience, 6(9):765-769.

Cox, S., Turnbull, I., Isaac, M., Townsend, D., and Smith Lyttle, B. (2012). Geology of Southern Victoria Land, Antarctica: scale 1:250,000. Lower Hutt: GNS Science. Institute of Geological \& Nuclear Sciences. 1:25,000 geological map 22. 135 p. +1 folded map.

Crary, A. P. (1966). Mechanism for fiord formation indicated by studies of an ice-covered inlet. Geological Society of America Bulletin, 77:911-930.

Cuffey, K., Conway, H., Gades, A., Hallet, B., Lorrain, R., Severinghaus, J., Steig, E., Vaughn, B., and White, J. (2000). Entrainment at cold glacier beds. Geology, 28(4):351-354.

Cuffey, K., Conway, H., Hallet, B., Gades, A., and Raymond, C. (1999). Interfacial water in polar glaciers and glacier sliding at $-17{ }^{\circ} \mathrm{c}$. Geophysical Research Letters, 26(6):751-754.

Cuffey, K. and Paterson, W. S. B. (2010). The Physics of Glaciers. Oxford, ButterworthHeinemann.

Cunningham, W. L., Leventer, A., Andrews, J. T., Jennings, A. E., and Licht, K. J. (1999). Late Pleistocene-Holocene marine conditions in the Ross Sea, Antarctica: evidence from the diatom record. The Holocene, 9(2):129-139.

Darvill, C. M. (2013). Cosmogenic nuclide analysis. In Geomorphological Techniques (Online Edition). British Society for Geomorphology.

Davies, B. J., Golledge, N. R., Glasser, N. F., Carrivick, J. L., Ligtenberg, S. R., Barrand, N. E., van den Broeke, M. R., Hambrey, M. J., and Smellie, J. L. (2014). Modelled glacier response to centennial temperature and precipitation trends on the Antarctic Peninsula. Nature Climate Change. 
Dawber, M. and Powell, R. D. (1997). Epifaunal distributions at Antarctic marine-ending glaciers; iniiň́Cuences of ice dynamics and sedimentation. In Ricci, C. A., editor, The Antarctic Region; Geological Evolution and Processes: Proceedings of the VII International Symposium on Antarctic Earth Sciences, Siena, Italy, pages 875-884.

De Boer, B., Van de Wal, R., Lourens, L., Bintanja, R., and Reerink, T. (2013). A continuous simulation of global ice volume over the past 1 million years with 3-D ice-sheet models. Climate dynamics, 41(5-6):1365-1384.

DeConto, R., Pollard, D., and Kowalewski, D. (2012). Modeling Antarctic ice sheet and climate variations during Marine Isotope Stage 31. Global and Planetary Change, 88-89:45-52.

DeConto, R. M. and Pollard, D. (2003). Rapid Cenozoic glaciation of Antarctica induced by declining atmospheric $\mathrm{CO}_{2}$. Nature, 421:245-249.

Delunel, R., Bourles, D. L., van der Beek, P. A., Schlunegger, F., Leya, I., Masarik, J., and Paquet, E. (2014). Snow shielding factors for cosmogenic nuclide dating inferred from long-term neutron detector monitoring. Quaternary Geochronology, 24:16-26.

Denton, G. and Marchant, D. (2000). The geologic basis for a reconstruction of a grounded ice sheet in McMurdo Sound, Antarctica, at the Last Glacial Maximum. Geografiska Annaler, 82A(2-3):167-211.

Denton, G. H., Bockheim, J. G., Wilson, S. C., and Stuiver, M. (1989). Late Wisconsin and early Holocene glacial history, inner Ross embayment, Antarctica. Quaternary Research, 31(2):151-182.

Denton, G. H. and Hughes, T. J. (2000). Reconstruction of the Ross ice drainage system, Antarctica, at the last glacial maximum. Geografiska Annaler, 82A(2):143-166.

Denton, G. H. and Hughes, T. J. (2002). Reconstructing the Antarctic ice sheet at the Last Glacial Maximum. Quaternary Science Reviews, 21(1-3):193-202.

Depoorter, M., Bamber, J., Griggs, J., Lenaerts, J., Ligtenberg, S., van den Broeke, M., and Moholdt, G. (2013). Calving fluxes and basal melt rates of Antarctic ice shelves. Nature.

Deschamps, P., Durand, N., Bard, E., Hamelin, B., Camoin, G., Thomas, A. L., Henderson, G. M., Okuno, J., and Yokoyama, Y. (2012). Ice-sheet collapse and sea-level rise at the Bølling warming 14,600 years ago. Nature, 483:559-564.

Desilets, D. and Zreda, M. (2003). Spatial and temporal distribution of secondary cosmicray nucleon intensities and applications to in situ cosmogenic dating. Earth and Planetary Science Letters, 206(1):21-42.

Desilets, D., Zreda, M., and Prabu, T. (2006). Extended scaling factors for in situ cosmogenic nuclides: new measurements at low latitude. Earth and Planetary Science Letters, 246(3):265-276.

Di Nicola, L., Baroni, C., Strasky, S., Salvatore, M. C., Schlüchter, C., Akçar, N., Kubik, P. W., and Wieler, R. (2012). Multiple cosmogenic nuclides document the stability of the East Antarctic Ice Sheet in northern Victoria Land since the Late Miocene (5-7 Ma). Quaternary Science Reviews, 57:85-94. 
Di Nicola, L., Strask, S., Schluechter, C., Salvatore, M. C., Akcar, N., Kubik, P. W., Christl, M., Kasper, H. U., Wieler, R., and Baroni, C. (2009). Multiple cosmogenic nuclides document complex Pleistocene exposure history of glacial drifts in Terra Nova Bay (northern Victoria Land, Antarctica). Quaternary Research, 71(1):83-92.

Dickinson, W. W., Schiller, M., Ditchburn, B. G., Graham, I. J., and Zondervan, A. (2012). Meteoric Be-10 from Sirius Group suggests high elevation McMurdo Dry Valleys permanently frozen since 6Ma. Earth and Planetary Science Letters, 355:13-19.

Dochat, T. M., Marchant, D. R., and Denton, G. H. (2000). Glacial geology of Cape Bird, Ross Island, Antarctica. Geografiska Annaler: Series A, Physical Geography, 82(2-3):237-247.

Domack, E., O’Brien, P., Harris, P., Taylor, F., Quilty, P. G., Santis, L. D., and Raker, B. (1998). Late Quaternary sediment facies in Prydz Bay, East Antarctica and their relationship to glacial advance onto the continental shelf. Antarctic Science, 10(03):236246.

Domack, E. W., Jacobson, E. A., Shipp, S., and Anderson, J. B. (1999). Late PleistoceneHolocene retreat of the West Antarctic Ice-Sheet system in the Ross Sea: Part 2 sedimentologic and stratigraphic signature. Geological Society of America Bulletin, 111(10):1517-1536.

Domack, E. W., Jull, A. T., and Nakao, S. (1991). Advance of East Antarctic outlet glaciers during the Hypsithermal: implications for the volume state of the Antarctic ice sheet under global warming. Geology, 19(11):1059-1062.

Dühnforth, M., Anderson, R. S., Ward, D., and Stock, G. M. (2010). Bedrock fracture control of glacial erosion processes and rates. Geology, 38(5):423-426.

Dunai, T. (2001). Influence of secular variation of the geomagnetic field on production rates of in situ produced cosmogenic nuclides. Earth and Planetary Science Letters, 193(1):197-212.

Dunai, T. J. (2000). Scaling factors for production rates of in situ produced cosmogenic nuclides: a critical reevaluation. Earth and Planetary Science Letters, 176(1):157-169.

Dunai, T. J. (2010). Cosmogenic Nuclides: Principles, concepts and applications in the Earth surface sciences. Cambridge University Press.

Dupont, T. and Alley, R. (2006). Role of small ice shelves in sea-level rise. Geophysical research letters, 33(9).

Dupont, T. K. and Alley, R. B. (2005). Assessment of the importance of ice-shelf buttressing to ice-sheet flow. Geophysical Research Letters, 32(4).

Dwyer, G. and Chandler, M. (2009). Mid-Pliocene sea level and continental ice volume based on coupled benthic $\mathrm{Mg} / \mathrm{Ca}$ palaeotemperatures and oxygen isotopes. Philosophical Transactions of the Royal Society A: Mathematical, Physical and Engineering Sciences, 367(1886):157.

Echelmeyer, K. and Zhongxiang, W. (1987). Direct observation of basal sliding and deformation of basal drift at sub-freezing temperatures. Journal of Glaciology, 33:8398 . 
Echelmeyer, K. A. and Kamb, B. (1986). Stress-gradient coupling in glacier flow: II. Longitudinal averaging of the flow response to small perturbations in ice thickness and surface slope. Journal of Glaciology, 32(111):285-298.

Elliot, D. H. (2013). The geological and tectonic evolution of the Transantarctic Mountains: a review. Geological Society, London, Special Publications, 381(1):7-35.

Enderlin, E., Howat, I., and Vieli, A. (2013). High sensitivity of tidewater outlet glacier dynamics to shape. The Cryosphere, 7(3):1007-1015.

Escutia, C., Bárcena, M., Lucchi, R., Romero, O., Ballegeer, A., Gonzalez, J., and Harwood, D. (2009). Circum-Antarctic warming events between 4 and 3.5 Ma recorded in marine sediments from the Prydz Bay (ODP Leg 188) and the Antarctic Peninsula (ODP Leg 178) margins. Global and Planetary Change, 69(3):170-184.

Fahnestock, M. A., Scambos, T. A., Bindschadler, R. A., and Kvaran, G. (2000). A millennium of variable ice flow recorded by the Ross Ice Shelf, Antarctica. Journal of Glaciology, 46(155):652-664.

Fairbanks, R. G. (1989). A 17,000-year glacio-eustatic sea level record: Influence of glacial melting rates on the Younger Dryas event and deep-ocean circulation. Nature, 342(6250):637-642.

Favier, L., Durand, G., Cornford, S., Gudmundsson, G., Gagliardini, O., Gillet-Chaulet, F., Zwinger, T., Payne, A., and Le Brocq, A. (2014). Retreat of Pine Island Glacier controlled by marine ice-sheet instability. Nature Climate Change.

Fedorov, A., Brierley, C., Lawrence, K., Liu, Z., Dekens, P., and Ravelo, A. (2013). Patterns and mechanisms of early Pliocene warmth. Nature, 496(7443):43-49.

Fink, D., McKelvey, B., Hambrey, M. J., Fabel, D., and Brown, R. (2006). Pleistocene deglaciation chronology of the Amery Oasis and Radok Lake, northern Prince Charles Mountains, Antarctica. Earth and Planetary Science Letters, 243(1):229-243.

Fleming, K., Johnston, P., Zwartz, D., Yokoyama, Y., Lambeck, K., and Chappell, J. (1998). Refining the eustatic sea-level curve since the Last Glacial Maximum using far- and intermediate-field sites. Earth and Planetary Science Letters, 163:327-342.

Fogwill, C. J., Hein, A. S., Bentley, M. J., and Sugden, D. E. (2012). Do blue-ice moraines in the Heritage Range show the West Antarctic ice sheet survived the last interglacial? Palaeogeography, Palaeoclimatology, Palaeoecology, (335-336):61-70.

Fowler, A. C. (2000). An instability mechanism for drumlin formation. Deformation of Glacial Materials, 176:307-319.

French, H. M. (2007). The periglacial environment. John Wiley \& Sons.

Fretwell, P., Pritchard, H. D., Vaughan, D. G., Bamber, J., Barrand, N., Bell, R., Bianchi, C., Bingham, R., Blankenship, D., and Casassa, G. (2013). Bedmap2: improved ice bed, surface and thickness datasets for Antarctica. Cryosphere, 7(1).

Frezzotti, M. (1997). Ice front fluctuation, iceberg calving flux and mass balance of Victoria Land glaciers. Antarctic Science, 9(01):61-73. 
Frezzotti, M., Tabacco, I. E., and Zirizzotti (2000). Ice discharge of eastern Dome C drainage area, Antarctica, determined from airbourne radar survey and satellite image analysis. Journal of Glaciology, 46(153):253-264.

Gagliardini, O., Zwinger, T., Gillet-Chaulet, F., Durand, G., Favier, L., Fleurian, B. d., Greve, R., Malinen, M., Martin, C., and Raback, P. (2013). Capabilities and performance of Elmer/Ice, a new generation ice-sheet model. Geoscientific Model Development Discussions, 6(1):1689-1741.

Gilks, W. R., Richardson, S., and Spiegelhalter, D. J. (1996). Introducing Markov Chain Monte Carlo. Markov chain Monte Carlo in practice, 1:19.

Gingele, F., Kuhn, G., Maus, B., Melles, M., and SchÃúne, T. (1997). Holocene ice retreat from the Lazarev Sea shelf, East Antarctica. Continental Shelf Research, 17(2):137163.

Giovinetto, M., Robinson, E. S., and Swinthinbank, C. W. M. (1964). The regime of the western part of the Ross Ice Shelf drainage system. Journal of Glaciology, 6(43):5568 .

Glasser, N. F. and Bennett, M. R. (2004). Glacial erosional landforms: origins and significance for palaeoglaciology. Progress in Physical Geography, 28(1):43-75.

Glasser, N. F. and Gudmundsson, G. H. (2012). Longitudinal surface structures (flowstripes) on Antarctic glaciers. The Cryosphere, 6:383-391.

Glasser, N. F. and Scambos, T. A. (2008). A structural glaciological analysis of the 2002 Larsen B ice-shelf collapse. Journal of Glaciology, 54(184):3-16.

Golledge, N. R., Fogwill, C. J., Mackintosh, A. N., and Buckley, K. M. (2012). Dynamics of the last glacial maximum Antarctic ice-sheet and its response to ocean forcing. Proceedings of the National Academy of Sciences, 109(40):16052-16056.

Golledge, N. R. and Levy, R. H. (2011). Geometry and dynamics of an East Antarctic Ice Sheet outlet glacier, under past and present climates. Journal of Geophysical Research (Earth Surface), 116:3025.

Golledge, N. R., Levy, R. H., McKay, R. M., Fogwill, C. J., White, D. A., Graham, A. G., Smith, J. A., Hillenbrand, C.-D., Licht, K. J., Denton, G. H., et al. (2013). Glaciology and geological signature of the Last Glacial Maximum Antarctic ice sheet. Quaternary Science Reviews, 78:225-247.

Golledge, N. R., Marsh, O. J., Rack, W., Braaten, D., and Jones, R. S. (2014a). Basal conditions of two Transantarctic Mountains outlet glaciers from observation-constrained diagnostic modelling. Journal of Glaciology, 60(223):855.

Golledge, N. R., Menviel, L., Carter, L., Fogwill, C. J., England, M. H., Cortese, G., and Levy, R. H. (2014b). Antarctic contribution to meltwater pulse 1A from reduced Southern Ocean overturning. Nature Communications, 5.

Gomez, N., Pollard, D., and Mitrovica, J. X. (2013). A 3-D coupled ice sheet-sea level model applied to Antarctica through the last $40 \mathrm{ky}$. Earth and Planetary Science Letters, 384:88-99. 
Gosse, J. C. and Phillips, F. M. (2001). Terrestrial in situ cosmogenic nuclides: theory and application. Quaternary Science Reviews, 20(14):1475-1560.

Graham, A. G., Larter, R. D., Gohl, K., Hillenbrand, C.-D., Smith, J. A., and Kuhn, G. (2009). Bedform signature of a West Antarctic palaeo-ice stream reveals a multi-temporal record of flow and substrate control. Quaternary Science Reviews, 28(25):2774-2793.

Graham, A. G. C., Larter, R. D., Gohl, K., Dowdeswell, J. A., Hillenbrand, C. D., Smith, J. A., Evans, J., Kuhn, G., and Deen, T. (2010). Flow and retreat of the Late Quaternary Pine Island-Thwaites palaeo-ice stream, West Antarctica. Journal of Geophysical Research, 115:F03025.

Granger, D. E., Lifton, N. A., and Willenbring, J. K. (2013). A cosmic trip: 25 years of cosmogenic nuclides in geology. Geological Society of America Bulletin, 125(910):1379-1402.

Greenwood, S. L., Gyllencreutz, R., Jakobsson, M., and Anderson, J. B. (2012). Ice-flow switching and east/west antarctic ice sheet roles in glaciation of the western ross sea. Geological Society of America Bulletin, 124(11-12):1736-1749.

Gregoire, L. J., Payne, A. J., and Valdes, P. J. (2012). Deglacial rapid sea level rises caused by ice-sheet saddle collapses. Nature, 487:219-223.

Grimm, E. (1990). TILIA and TILIA.GRAPH: PC spreadsheet and graphics software for pollen data. INQUA Commission for the Study of the Holocene working group on data handling methods newsletter, 4:5-7.

Gunn, B. M. and Warren, G. (1962). Geology of Victoria Land between the Mawson and Mulock Glaciers, Antarctica. Number 70-71. Trans-antarctic expedition Committee.

Guyodo, Y. and Valet, J.-P. (1999). Global changes in intensity of the Earth's magnetic field during the past $800 \mathrm{kyr}$. Nature, 399(6733):249-252.

Hall, B., Denton, G., and Hendy, C. (2000). Evidence from Taylor Valley for a grounded ice sheet in the Ross Sea, Antarctica. Geografiska Annaler Series A - Physical Geography, 82A(2-3):275-303.

Hall, B. L., Baroni, C., and Denton, G. H. (2004). Holocene relative sea-level history of the Southern Victoria Land Coast, Antarctica. Global and Planetary Change, 42(14):241-263.

Hall, B. L. and Denton, G. H. (1999). New relative sea-level curves for the southern Scott Coast, Antarctica: evidence for Holocene deglaciation of the western Ross Sea. Journal of Quaternary Science, 14(7):641-650.

Hall, B. L. and Denton, G. H. (2000). Radiocarbon chronology of Ross Sea drift, eastern Taylor Valley, Antarctica: Evidence for a grounded ice sheet in the Ross Sea at the last glacial maximum. Geografiska Annaler, 82A:305-336.

Hall, B. L., Henderson, G. M., Baroni, C., and Kellogg, T. B. (2010). Constant Holocene Southern-Ocean ${ }^{14} \mathrm{C}$ reservoir ages and ice-shelf flow rates. Earth and Planetary Science Letters, 296(1):115-123. 
Hall, K. (1997a). Observations on "cryoplanation" benches in Antarctica. Antarctic Science, 9(02):181-187.

Hall, K. (1997b). Rock temperatures and implications for cold region weathering. I: New data from Viking Valley, Alexander Island, Antarctica. Permafrost and Periglacial Processes, 8(1):69-90.

Hall, K. (1998). Nivation or cryoplanation: Different terms, same features? Polar Geography, 22(1):1-16.

Hall, K. and André, M.-F. (2001). New insights into rock weathering from high-frequency rock temperature data: an Antarctic study of weathering by thermal stress. Geomorphology, 41(1):23-35.

Hall, K., Thorn, C. E., Matsuoka, N., and Prick, A. (2002). Weathering in cold regions: some thoughts and perspectives. Progress in Physical Geography, 26(4):577-603.

Hallet, B. (1979). A theoretical model of glacial abrasion. Journal of Glaciology, 23:3950 .

Hanebuth, T., Stattegger, K., and Grootes, P. M. (2000). Rapid flooding of the Sunda Shelf: a late-glacial sea-level record. Science, 288(5468):1033-1035.

Hanna, E., Navarro, F. J., Pattyn, F., Domingues, C. M., Fettweis, X., Ivins, E. R., Nicholls, R. J., Ritz, C., Smith, B., Tulaczyk, S., et al. (2013). Ice-sheet mass balance and climate change. Nature, 498(7452):51-59.

Harig, C. and Simons, F. J. (2015). Accelerated West Antarctic ice mass loss continues to outpace East Antarctic gains. Earth and Planetary Science Letters, 415:134-141.

Harris, P. T., Brancolini, G., Armand, L., Busetti, M., Beaman, R. J., Giorgetti, G., Presti, M., and Trincardi, F. (2001). Continental shelf drift deposit indicates non-steady state Antarctic bottom water production in the Holocene. Marine Geology, 179(1):1-8.

Haywood, A., Hill, D., Dolan, A., Otto-Bliesner, B., Bragg, F., Chan, W.-L., Chandler, M., Contoux, C., Dowsett, H., Jost, A., et al. (2013). Large-scale features of Pliocene climate: results from the Pliocene Model Intercomparison Project. Climate of the Past, 9:191-209.

Heegaard, E., Birks, H. J. B., and Telford, R. J. (2005). Relationships between calibrated ages and depth in stratigraphical sequences: an estimation procedure by mixed-effect regression. The Holocene, 15(4):612-618.

Hein, A. S., Fogwill, C. J., Sugden, D. E., and Xu, S. (2011). Glacial/interglacial icestream stability in the Weddell Sea embayment, Antarctica. Earth and Planetary Science Letters, 307:211-221.

Hein, A. S., Fogwill, C. J., Sugden, D. E., and Xu, S. (2014). Geological scatter of cosmogenic-nuclide exposure ages in the Shackleton Range, Antarctica: Implications for glacial history. Quaternary Geochronology, 19:52-66.

Hillenbrand, C.-D., Bentley, M. J., Stolldorf, T. D., Hein, A. S., Kuhn, G., Graham, A. G., Fogwill, C. J., Kristoffersen, Y., Smith, J. A., and Anderson, J. B. (2014). Reconstruction of changes in the Weddell Sea sector of the Antarctic Ice Sheet since the Last Glacial Maximum. Quaternary Science Reviews. 
Hillenbrand, C.-D., Larter, R. D., Dowdeswell, J., Ehrmann, W., O'Cofaigh, C., Benetti, S., Graham, A. G., and Grobe, H. (2010). The sedimentary legacy of a palaeo-ice stream on the shelf of the southern Bellingshausen Sea: Clues to West Antarctic glacial history during the Late Quaternary. Quaternary Science Reviews, 29(19):2741-2763.

Hillenbrand, C.-D., Melles, M., Kuhn, G., and Larter, R. D. (2012). Marine geological constraints for the grounding-line position of the Antarctic Ice Sheet on the southern Weddell Sea shelf at the Last Glacial Maximum. Quaternary Science Reviews, 32:2547.

Hindmarsh, R. (1998). Ice-stream surface texture, sticky spots, waves and breathers: the coupled flow of ice, till and water. Journal of Glaciology, 44(148):589-614.

Hindmarsh, R. C. (2001). Influence of channelling on heating in ice-sheet flows. Geophysical research letters, 28(19):3681-3684.

Hindmarsh, R. C. and Le Meur, E. (2001). Dynamical processes involved in the retreat of marine ice sheets. Journal of Glaciology, 47(157):271-282.

Hock, R. (2003). Temperature index melt modelling in mountain areas. Journal of Hydrology, 282(1):104-115.

Holbourn, A., Kuhnt, W., Schulz, M., and Erlenkeuser, H. (2005). Impacts of orbital forcing and atmospheric carbon dioxide on Miocene ice-sheet expansion. Nature, 438(7067):483-487.

Holland, P. R., Jenkins, A., and Holland, D. M. (2008). The response of ice shelf basal melting to variations in ocean temperature. Journal of Climate, 21(11):2558-2572.

Howat, I. M., Joughin, I., and Scambos, T. A. (2007). Rapid changes in ice discharge from Greenland outlet glaciers. Science, 315(5818):1559-1561.

Hubbard, A. (2006). The validation and sensitivity of a model of the Icelandic ice sheet. Quaternary Science Reviews, 25(17):2297-2313.

Huber, M. and Nof, D. (2006). The ocean circulation in the southern hemisphere and its climatic impacts in the Eocene. Palaeogeography, Palaeoclimatology, Palaeoecology, 231(1):9-28.

Hulbe, C. and Fahnestock, M. (2007). Century-scale discharge stagnation and reactivation of the Ross ice streams, West Antarctica. Journal of Geophysical Research: Earth Surface (2003-2012), 112(F3).

Hulbe, C. L., Scambos, T. A., Youngberg, T., and Lamb, A. K. (2008). Patterns of glacier response to disintegration of the Larsen B ice shelf, Antarctic Peninsula. Global and Planetary Change, 63(1):1-8.

Humbert, A., Greve, R., and Hutter, K. (2005). Parameter sensitivity studies for the ice flow of the Ross Ice Shelf, Antarctica. Journal of Geophysical Research - Earth Science, 110(F4).

Huybers, P. (2006). Early Pleistocene glacial cycles and the integrated summer insolation forcing. Science, 313(5786):508-511.

Huybers, P. (2009). Antarctica's orbital beat. Science, 325(5944):1085-1086. 
Huybers, P. and Denton, G. (2008). Antarctic temperature at orbital timescales controlled by local summer duration. Nature Geoscience, 1(11):787-792.

Huybrechts, P. (2002). Sea-level changes at the LGM from ice-dynamic reconstructions of the Greenland and Antarctic ice sheets during the glacial cycles. Quaternary Science Reviews, 21(1-3):203-231.

Huybrechts, P. and Oerlemans, J. (1990). Response of the Antarctic ice sheet to future greenhouse warming. Climate Dynamics, 5(2):93-102.

Ivins, E. R. and James, T. S. (2005). Antarctic glacial isostatic adjustment: a new assessment. Antarctic Science, 17(04):541-553.

Ivins, E. R., Watkins, M. M., Yuan, D. N., Dietrich, R., Casassa, G., and Rulke, A. (2011). On-land ice loss and glacial isostatic adjustment at the Drake Passage: 2003-2009. Journal of Geophysical Research-Solid Earth, 116:24 (B02403).

Ivy-Ochs, S. and Kober, F. (2008). Surface exposure dating with cosmogenic nuclides. Eiszeitalter und Gegenwart, 57(1/2):179-209.

Ivy-Ochs, S., Schlüchter, C., Kubik, P. W., Dittrich-Hannen, B., and Beer, J. (1995). Minimum ${ }^{10} \mathrm{Be}$ exposure ages of early Pliocene for the Table Mountain plateau and the Sirius Group at Mount Fleming, dry valleys, Antarctica. Geology, 23(11):1007-1010.

Jacobs, S. S., Jenkins, A., Giulivi, C. F., and Dutrieux, P. (2011). Stronger ocean circulation and increased melting under Pine Island Glacier ice shelf. Nature Geoscience, 4(8):519-523.

Jamieson, S., Sugden, D., and Hulton, N. (2010). The evolution of the subglacial landscape of Antarctica. Earth and Planetary Science Letters, 293:1-27.

Jamieson, S. S., Vieli, A., Livingstone, S. J., O'Cofaigh, C., Stokes, C., Hillenbrand, C.D., and Dowdeswell, J. A. (2012). Ice-stream stability on a reverse bed slope. Nature Geoscience, 5(11):799-802.

Jamieson, S. S., Vieli, A., O'Cofaigh, C., Stokes, C. R., Livingstone, S. J., and Hillenbrand, C.-D. (2014). Understanding controls on rapid ice-stream retreat during the last deglaciation of Marguerite Bay, Antarctica, using a numerical model. Journal of Geophysical Research: Earth Surface, 119(2):247-263.

Jenkins, A., Dutrieux, P., Jacobs, S. S., McPhail, S. D., Perrett, J. R., Webb, A. T., and White, D. (2010). Observations beneath Pine Island Glacier in West Antarctica and implications for its retreat. Nature Geoscience, 3(7):468-472.

Johnson, J. S., Bentley, M. J., and Gohl, K. (2008). First exposure ages from the Amundsen Sea embayment, West Antarctica: The late quaternary context for recent thinning of Pine Island, Smith, and Pope Glaciers. Geology, 36(3):223-226.

Johnson, J. S., Bentley, M. J., Smith, J. A., Finkel, R., Rood, D., Gohl, K., Balco, G., Larter, R. D., and Schaefer, J. (2014). Rapid thinning of Pine Island Glacier in the early Holocene. Science, 343(6174):999-1001.

Johnson, J. S., Everest, J. D., Leat, P. T., Golledge, N. R., Rood, D. H., and Stuart, F. M. (2012). The deglacial history of NW Alexander Island, Antarctica, from surface exposure dating. Quaternary Research, 77(2):273-280. 
Johnson, J. V. and Staiger, J. W. (2007). Modeling long-term stability of the Ferrar Glacier, East Antarctica: Implications for interpreting cosmogenic nuclide inheritance. Journal of Geophysical Research, 112:F03S30.

Joughin, I. and Alley, R. B. (2011). Stability of the West Antarctic ice sheet in a warming world. Nature Geoscience, 4:506 - 513.

Joughin, I., Smith, B. E., and Medley, B. (2014). Marine Ice Sheet Collapse Potentially Under Way for the Thwaites Glacier Basin, West Antarctica. Science, 344(6185):735738.

Jouzel, J., Masson-Delmotte, V., Cattani, O., Dreyfus, G., Falourd, S., Hoffmann, G., Minster, B., Nouet, J., Barnola, J.-M., and Chappellaz, J. (2007). Orbital and millennial Antarctic climate variability over the past 800,000 years. Science, 317(5839):793-796.

Joy, K., Fink, D., Storey, B., and Atkins, C. (2014). A 2 million year glacial chronology of the Hatherton Glacier, Antarctica and implications for the size of the East Antarctic Ice Sheet at the Last Glacial Maximum. Quaternary Science Reviews, 83:46-57.

Kamb, B. (2001). Basal zone of the West Antarctic ice streams and its role in lubrication of their rapid motion. The West Antarctic ice sheet: behavior and environment, pages 157-199.

Kamb, B. and Echelmeyer, K. A. (1986). Stress-gradient coupling in glacier flow: I. Longitudinal averaging of the influence of ice thickness and surface slope. Journal of Glaciology, 32(111):267-284.

Kaplan, M. R., Strelin, J. A., Schaefer, J. M., Denton, G. H., Finkel, R. C., Schwartz, R., Putnam, A. E., Vandergoes, M. J., Goehring, B. M., and Travis, S. G. (2011). Insitu cosmogenic ${ }^{10} \mathrm{Be}$ production rate at Lago Argentino, Patagonia: Implications for late-glacial climate chronology. Earth and Planetary Science Letters, 309(1):21-32.

Kavanaugh, J. L., Cuffey, K., Morse, D. L., Conway, H., and Rignot, E. (2009). Dynamics and mass balance of Taylor Glacier, Antarctica: 1. geometry and surface velocities. Journal of Geophysical Research, 114:F04010.

Kavanaugh, J. L. and Cuffey, K. M. (2009). Dynamics and mass balance of Taylor Glacier, Antarctica: 2. Force balance and longitudinal coupling. Journal of Geophysical Research - Earth Surface, 114.

Kennett, J. P. (1977). Cenozoic evolution of Antarctic glaciation, the circum-Antarctic Ocean, and their impact on global paleoceanography. Journal of Geophysical Research, 82(27):3843-3860.

King, M. A., Bingham, R. J., Moore, P., Whitehouse, P. L., Bentley, M. J., and Milne, G. A. (2012). Lower satellite-gravimetry estimates of Antarctic sea-level contribution. Nature, 491(7425):586-589.

Kirshner, A. E., Anderson, J. B., Jakobsson, M., O'Regan, M., Majewski, W., and Nitsche, F. O. (2012). Post-LGM deglaciation in Pine Island Bay, West Antarctica. Quaternary Science Reviews, 38:11-26.

Klages, J. P., Kuhn, G., Hillenbrand, C.-D., Graham, A., Smith, J., Larter, R., and Gohl, K. (2013). First geomorphological record and glacial history of an inter-ice stream ridge on the West Antarctic continental shelf. Quaternary Science Reviews, 61:47-61. 
Klages, J. P., Kuhn, G., Hillenbrand, C.-D., Graham, A. G., Smith, J. A., Larter, R. D., Gohl, K., and Wacker, L. (2014). Retreat of the West Antarctic Ice Sheet from the western Amundsen Sea shelf at a pre-or early LGM stage. Quaternary Science Reviews, 91:1-15.

Klein, J., Giegengack, R., Middleton, R., Sharma, P., Underwood, J., and Weeks, R. (1986). Revealing histories of exposure using in situ produced ${ }^{26} \mathrm{Al}$ and ${ }^{10} \mathrm{Be}$ in Libyan desert glass. Radiocarbon, 28(2A):547-555.

Kopp, R. E., Simons, F. J., Mitrovica, J. X., Maloof, A. C., and Oppenheimer, M. (2009). Probabilistic assessment of sea level during the last interglacial stage. $\mathrm{Na}$ ture, 462(7275):863-867.

Korte, M. and Constable, C. (2005). The geomagnetic dipole moment over the last 7000 years - new results from a global model. Earth and Planetary Science Letters, 236(1):348-358.

Kristoffersen, Y., Strand, K., Vorren, T., Harwood, D., and Webb, P. (2000a). Pilot shallow drilling on the continental shelf, Dronning Maud Land, Antarctica. Antarctic Science, 12(04):463-470.

Kristoffersen, Y., Winterhalter, B., and Solheim, A. (2000b). Shelf progradation on a glaciated continental margin, Queen Maud Land, Antarctica. Marine Geology, 165(1):109-122.

Laj, C., Kissel, C., and Beer, J. (2004). High Resolution Global Paleointensity Stack Since $75 \mathrm{kyr}$ (GLOPIS-75) Calibrated to Absolute Values. Timescales of the Paleomagnetic Field, pages 255-265.

Lal, D. (1988). In situ-produced cosmogenic isotopes in terrestrial rocks. Annual Review of Earth and Planetary Sciences, 16:355-388.

Lal, D. (1991). Cosmic ray labeling of erosion surfaces: in situ nuclide production rates and erosion models. Earth and Planetary Science Letters, 104(2):424-439.

Lal, D. and Peters, B. (1967). Cosmic ray produced radioactivity on the earth. In Kosmische Strahlung II/Cosmic Rays II, pages 551-612. Springer.

Lambeck, K., Rouby, H., Purcell, A., Sun, Y., and Sambridge, M. (2014). Sea level and global ice volumes from the Last Glacial Maximum to the Holocene. Proceedings of the National Academy of Sciences, page 201411762.

Larter, R. D., Anderson, J. B., Graham, A. G., Gohl, K., Hillenbrand, C.-D., Jakobsson, M., Johnson, J. S., Kuhn, G., Nitsche, F. O., and Smith, J. A. (2014). Reconstruction of changes in the Amundsen Sea and Bellingshausen Sea sector of the West Antarctic Ice Sheet since the Last Glacial Maximum. Quaternary Science Reviews.

Lenaerts, J. T. M., van den Broeke, M. R., van de Berg, W. J., van Meijgaard, E., and Munneke, P. K. (2012). A new, high-resolution surface mass balance map of Antarctica (1979-2010) based on regional atmospheric climate modeling. Geophysical Research Letters, 39(L04501).

Leventer, A., Domack, E., Dunbar, R., Pike, J., Stickley, C., Maddison, E., Brachfeld, S., Manley, P., and McClennen, C. (2006). Marine sediment record from the East Antarctic margin reveals dynamics of ice sheet recession. GSA Today, 16(12):4. 
Levy, R., Cody, R., Crampton, J., Fielding, C., Golledge, N., Harwood, D., Henrys, S., McKay, R., Naish, T., Ohneiser, C., Wilson, G., Wilson, T., and Winter, D. (2012). Late Neogene climate and glacial history of the Southern Victoria Land coast from integrated drill core, seismic and outcrop data. Global and Planetary Change, 80-81:61-84.

Lewis, A., Marchant, D., Ashworth, A., Hemming, S., and Machlus, M. (2007). Major middle Miocene global climate change: Evidence from East Antarctica and the Transantarctic Mountains. Geological Society of America Bulletin, 119(11-12):1449_ 1461.

Lewis, A. R., Marchant, D. R., Ashworth, A. C., Hedenäs, L., Hemming, S. R., Johnson, J. V., Leng, M. J., Machlus, M. L., Newton, A. E., Raine, J. I., et al. (2008). MidMiocene cooling and the extinction of tundra in continental Antarctica. Proceedings of the National Academy of Sciences, 105(31):10676-10680.

Lewis, A. R., Marchant, D. R., Kowalewski, D. E., Baldwin, S. L., and Webb, L. E. (2006). The age and origin of the Labyrinth, western Dry Valleys, Antarctica: Evidence for extensive middle Miocene subglacial floods and freshwater discharge to the Southern Ocean. Geology, 34(7):513-516.

Licht, K., Jennings, A., Andrews, J., and Williams, K. (1996). Chronology of late Wisconsin ice retreat from the western Ross Sea, Antarctica. Geology, 24:223-226.

Licht, K. J., Lederer, J. R., and Swope, R. J. (2005). Provenance of LGM glacial till (sand fraction) across the Ross embayment, Antarctica. Quaternary Science Reviews, 24:1499-1520.

Lifton, N. (2014). Comparing time-dependent geomagnetic and atmospheric effects on cosmogenic nuclide production rate scaling. American Geophysical Union.

Lifton, N., Sato, T., and Dunai, T. J. (2014). Scaling in situ cosmogenic nuclide production rates using analytical approximations to atmospheric cosmic-ray fluxes. Earth and Planetary Science Letters, 386:149-160.

Lifton, N. A., Bieber, J. W., Clem, J. M., Duldig, M. L., Evenson, P., Humble, J. E., and Pyle, R. (2005). Addressing solar modulation and long-term uncertainties in scaling secondary cosmic rays for in situ cosmogenic nuclide applications. Earth and Planetary Science Letters, 239(1):140-161.

Lilly, K., Fink, D., Fabel, D., and Lambeck, K. (2010). Pleistocene dynamics of the interior East Antarctic ice sheet. Geology, 38(8):703-706.

Lisiecki, L. E. and Raymo, M. E. (2005). A Pliocene-Pleistocene stack of 57 globally distributed benthic $\delta^{18} \mathrm{O}$ records. Paleoceanography, 20(1).

Liu, J. P. and Milliman, J. D. (2004). Reconsidering melt-water pulses 1A and 1B: global impacts of rapid sea-level rise. Journal of Ocean University of China, 3(2):183-190.

Liu, Z., Otto-Bliesner, B. L., He, F., Brady, E. C., Tomas, R., Clark, P. U., Carlson, A. E., Lynch-Stieglitz, J., Curry, W., Brook, E., Erickson, D., Jacob, R., Kutzbach, J., and Cheng, J. (2009). Transient Simulation of Last Deglaciation with a New Mechanism for Bølling-Allerød Warming. Science, 325(5938):310-314.

Livingstone, S., O'Cofaigh, C., C.R., S., Hillenbrand, C.-D., Vieli, A., and Jamieson, S. (2012). Antarctic palaeo-ice streams. Earth-Science Reviews, 111:90-128. 
Lloyd-Davies, M. T., Atkins, C. B., van der Meer, J. J. M., Barrett, P. J., and Hicock, S. R. (2009). Evidence for cold-based glacial activity in the Allan Hills, Antarctica. Quaternary Science Reviews, 28(27-28):3124-3137.

Lowe, A. L. and Anderson, J. B. (2002). Reconstruction of the West Antarctic ice sheet in Pine Island Bay during the Last Glacial Maximum and its subsequent retreat history. Quaternary Science Reviews, 21(16):1879-1897.

MacAyeal, D. R. (1989). Large-scale ice flow over a viscous basal sediment: Theory and application to ice stream B, Antarctica. Journal of Geophysical Research: Solid Earth (1978-2012), 94(B4):4071-4087.

Mackintosh, A., Golledge, N., Domack, E., Dunbar, R., Leventer, A., White, D., Pollard, D., DeConto, R., Fink, D., Zwartz, D., Gore, D., and Lavoie, C. (2011). Retreat of the East Antarctic ice sheet during the last glacial termination. Nature Geoscience, 4(3):195-202.

Mackintosh, A., White, D., Fink, D., Gore, D. B., Pickard, J., and Fanning, P. C. (2007). Exposure ages from mountain dipsticks in Mac. Robertson Land, East Antarctica, indicate little change in ice-sheet thickness since the Last Glacial Maximum. Geology, 35(6):551-554.

Mackintosh, A. N., Verleyen, E., O’Brien, P. E., White, D. A., Jones, R. S., McKay, R., Dunbar, R., Gore, D. B., Fink, D., and Post, A. L. (2014). Retreat history of the East Antarctic Ice Sheet since the Last Glacial Maximum. Quaternary Science Reviews.

Macpherson, A. J. (1987). The Mackay Glacier / Granite Harbour system (Ross Dependency, Antarctica). Unpublished Ph.D thesis, Victoria University of Wellington.

Marcott, S. A., Bauska, T. K., Buizert, C., Steig, E. J., Rosen, J. L., Cuffey, K. M., Fudge, T., Severinghaus, J. P., Ahn, J., Kalk, M. L., et al. (2014). Centennial-scale changes in the global carbon cycle during the last deglaciation. Nature, 514(7524):616-619.

Maris, M., van Wessem, J., van de Berg, W., de Boer, B., and Oerlemans, J. (2014). A model study of the effect of climate and sea-level change on the evolution of the Antarctic Ice Sheet from the Last Glacial Maximum to 2100. Climate Dynamics, pages $1-15$.

Marshall, S. J. and Clark, P. U. (2002). Basal temperature evolution of North American ice sheets and implications for the 100-kyr cycle. Geophysical Research Letters, 29(24):67-1.

Marzeion, B., Cogley, J. G., Richter, K., and Parkes, D. (2014). Attribution of global glacier mass loss to anthropogenic and natural causes. Science, 345(6199):919-921.

Masarik, J. and Beer, J. (1999). Simulation of particle fluxes and cosmogenic nuclide production in the Earth's atmosphere. Journal of Geophysical Research: Atmospheres (1984-2012), 104(D10):12099-12111.

Masarik, J. and Reedy, R. C. (1995). Terrestrial cosmogenic-nuclide production systematics calculated from numerical simulations. Earth and Planetary Science Letters, 136(3):381-395. 
Masson-Delmotte, V., Schulz, M., Abe-Ouchi, A., Beer, J., Ganopolski, A., González Rouco, J., Jansen, E., Lambeck, K., Luterbacher, J., Naish, T., et al. (2013). Information from paleoclimate archives. In Climate Change 2013: The Physical Science Basis. Contribution of Working Group I to the Fifth Assessment Report of the Intergovernmental Panel on Climate Change, pages 383-464. Cambridge University Press, Cambridge, United Kingdom.

Matsuoka, N. (1991). A model of the rate of frost shattering: application to field data from Japan, Svalbard and Antarctica. Permafrost and Periglacial Processes, 2(4):271-281.

Matsuoka, N. (1995). Rock weathering processes and landform development in the Sør Rondane Mountains, Antarctica. Geomorphology, 12:323-339.

Matsuoka, N. and Hirakawa, K. (2000). Solifluction resulting from one-sided and twosided freezing: field data from Svalbard. Polar geoscience, 13:187-201.

McCrae, I. R. (1984). A summary of glaciological measurements made between 1960 and 1984 on the McMurdo Ice Shelf, Antarctica, volume 360. University of Auckland, Department of Theoretical and Applied Mechanics.

McKay, R., Browne, G., Carter, L., Cowan, E., Dunbar, G., Krissek, L., Naish, T., Powell, R., Reed, J., Talarico, F., et al. (2009). The stratigraphic signature of the late Cenozoic Antarctic Ice Sheets in the Ross Embayment. Geological Society of America Bulletin, 121(11-12):1537-1561.

McKay, R., Naish, T., Carter, L., Riesselman, C., Dunbar, R., Sjunneskog, C., Winter, D., Sangiorgi, F., Warren, C., Pagani, M., Schouten, S., Willmott, V., Levy, R., DeConto, R., and Powell, R. D. (2012). Antarctic and Southern Ocean influences on Late Pliocene global cooling. Proceedings of the National Academy of Sciences, 109:6423-6428.

McKay, R., Naish, T., Powell, R., Barrett, P., Scherer, R., Talarico, F., Kyle, P., Monien, D., Kuhn, G., Jackolski, C., and Williams, T. (2012). Pleistocene variability of Antarctic Ice Sheet extent in the Ross Embayment. Quaternary Science Reviews, 34:93-112.

Mckay, R. M., Dunbar, G. B., Naish, T. R., Barrett, P. J., Carter, L., and Harper, M. (2008). Retreat history of the Ross Ice Sheet (Shelf) since the Last Glacial Maximum from deep-basin sediment cores around Ross Island. Palaeogeography, Palaeoclimatology, Palaeoecology, 260:245-261.

Ménabréaz, L., Thouveny, N., Bourlès, D., Deschamps, P., Hamelin, B., and Demory, F. (2011). The Laschamp geomagnetic dipole low expressed as a cosmogenic ${ }^{10} \mathrm{Be}$ atmospheric overproduction at $\sim 41 \mathrm{ka}$. Earth and Planetary Science Letters, 312(3):305317.

Mengel, M. and Levermann, A. (2014). Ice plug prevents irreversible discharge from East Antarctica. Nature Climate Change.

Mercer, J. H. (1978). West Antarctic ice sheet and CO2 greenhouse effect: a threat of disaster. Nature, 271(5643):321-325.

Miles, B., Stokes, C., Vieli, A., and Cox, N. (2013). Rapid, climate-driven changes in outlet glaciers on the Pacific coast of East Antarctica. Nature, 500(7464):563-566. 
Miller, G. H., Wolfe, A. P., Steig, E. J., Sauer, P. E., Kaplan, M. R., and Briner, J. P. (2002). The Goldilocks dilemma: big ice, little ice, or "just-right" ice in the Eastern Canadian Arctic. Quaternary Science Reviews, 21(1):33-48.

Miller, K. G., Kominz, M. A., Browning, J. V., Wright, J. D., Mountain, G. S., Katz, M. E., Sugarman, P. J., Cramer, B. S., Christie-Blick, N., and Pekar, S. F. (2005). The Phanerozoic record of global sea-level change. science, 310(5752):1293-1298.

Miller, K. G., Wright, J. D., Browning, J. V., Kulpecz, A., Kominz, M., Naish, T. R., Cramer, B. S., Rosenthal, Y., Peltier, W. R., and Sosdian, S. (2012). High tide of the warm Pliocene: Implications of global sea level for Antarctic deglaciation. Geology, 40(5):407-410.

Möller, P. (1995). Subrecent moraine ridge formation on Cuff Cape, Victoria Land, Antarctica. Geografiska Annaler. Series A. Physical Geography, 77(1/2):83-94.

Monnin, E., Steig, E. J., Siegenthaler, U., Kawamura, K., Schwander, J., Stauffer, B., Stocker, T. F., Morse, D. L., Barnola, J.-M., Bellier, B., et al. (2004). Evidence for substantial accumulation rate variability in Antarctica during the Holocene, through synchronization of $\mathrm{CO}_{2}$ in the Taylor Dome, Dome $\mathrm{C}$ and DML ice cores. Earth and Planetary Science Letters, 224(1):45-54.

Morse, D., Waddington, E., and Steig, E. (1998). Ice age storm trajectories inferred from radar stratigraphy at Taylor Dome, Antarctica. Geophysical Research Letters, 25(17):3383-3386.

Morse, D. L., Waddington, E. D., Marshall, H. P., Neumann, T. A., Steig, E. J., Dibb, J. E., Winebrenner, D. P., and Arthern, R. J. (1999). Accumulation rate measurements at Taylor Dome, East Antarctica: Techniques and Strategies for mass balance measurements in polar environments. Geografiska Annaler.

Mulvaney, R., Abram, N. J., Hindmarsh, R. C., Arrowsmith, C., Fleet, L., Triest, J., Sime, L. C., Alemany, O., and Foord, S. (2012). Recent Antarctic Peninsula warming relative to Holocene climate and ice-shelf history. Nature, 489(7414):141-144.

Naish, T., Powell, R., Levy, R., Wilson, G., Scherer, R., Talarico, F., Krissek, L., Niessen, F., Pompilio, M., Wilson, T., Carter, L., DeConto, R., Huybers, P., McKay, R., Pollard, D., Ross, J., Winter, D., Barrett, P., Browne, G., Cody, R., Cowan, E., Crampton, J., Dunbar, G., Dunbar, N., Florindo, F., Gebhardt, C., Graham, I., Hannah, M., Hansaraj, D., Harwood, D., Helling, D., Henrys, S., Hinnov, L., Kuhn, G., Kyle, P., Laufer, A., Maffioli, P., Magens, D., Mandernack, K., McIntosh, W., Millan, C., Morin, R., Ohneiser, C., Paulsen, T., Persico, D., Raine, I., Reed, J., Riesselman, C., Sagnotti, L., Schmitt, D., Sjunneskog, C., Strong, P., Taviani, M., Vogel, S., Wilch, T., and Williams, T. (2009). Obliquity-paced Pliocene West Antarctic ice sheet oscillations. Nature, 458:322-328.

Naish, T. R., Woolfe, K. J., Barrett, P. J., Wilson, G. S., Atkins, C., Bohaty, S. M., Bücker, C. J., Claps, M., Davey, F. J., Dunbar, G. B., et al. (2001). Orbitally induced oscillations in the East Antarctic ice sheet at the Oligocene/Miocene boundary. Nature, 413(6857):719-723.

Nick, F., Van der Veen, C., Vieli, A., and Benn, D. (2010). A physically based calving model applied to marine outlet glaciers and implications for the glacier dynamics. Journal of Glaciology, 56(199):781-794. 
Nick, F. M., Vieli, A., Howat, I. M., and Joughin, I. (2009). Large-scale changes in Greenland outlet glacier dynamics triggered at the terminus. Nature Geoscience, 2(2):110114.

Nishiizumi, K. (2004). Preparation of ${ }^{26} \mathrm{Al}$ AMS standards. Nuclear Instruments and Methods in Physics Research Section B: Beam Interactions with Materials and Atoms, 223:388-392.

Nishiizumi, K., Imamura, M., Caffee, M. W., Southon, J. R., Finkel, R. C., and McAninch, J. (2007). Absolute calibration of ${ }^{10}$ Be AMS standards. Nuclear Instruments and Methods in Physics Research Section B: Beam Interactions with Materials and Atoms, 258(2):403-413.

Nishiizumi, K., Winterer, E., Kohl, C., Klein, J., Middleton, R., Lal, D., and Arnold, J. (1989). Cosmic ray production rates of ${ }^{10} \mathrm{Be}$ and ${ }^{26} \mathrm{Al}$ in quartz from glacially polished rocks. Journal of Geophysical Research: Solid Earth (1978-2012), 94(B12):1790717915.

Norton, K. P., von Blanckenburg, F., Schlunegger, F., Schwab, M., and Kubik, P. W. (2008). Cosmogenic nuclide-based investigation of spatial erosion and hillslope channel coupling in the transient foreland of the Swiss Alps. Geomorphology, 95(3-4):474486.

O'Cofaigh, C., Davies, B. J., Livingstone, S. J., Smith, J. A., Johnson, J. S., Hocking, E. P., Hodgson, D. A., Anderson, J. B., Bentley, M. J., and Canals, M. (2014). Reconstruction of ice-sheet changes in the Antarctic Peninsula since the Last Glacial Maximum. Quaternary Science Reviews, 100:87-110.

Oerlemans, J. (1997). A flowline model for Nigardsbreen, Norway: projection of future glacier length based on dynamic calibration with the historic record. Journal of glaciology, 24:382-389.

Oerlemans, J., Anderson, B., Hubbard, A., Huybrechts, P., Johannesson, T., Knap, W., Schmeits, M., Stroeven, A., Van de Wal, R., Wallinga, J., et al. (1998). Modelling the response of glaciers to climate warming. Climate Dynamics, 14(4):267-274.

Oerlemans, J. et al. (2013). A note on the water budget of temperate glaciers. The Cryosphere, 7:1557-1564.

O’Neel, S., Pfeffer, W. T., Krimmel, R., and Meier, M. (2005). Evolving force balance at Columbia Glacier, Alaska, during its rapid retreat. Journal of Geophysical Research: Earth Surface (2003-2012), 110(F3).

Orombelli, G., Baroni, C., and Denton, G. H. (1990). Late Cenozoic glacial history of the Terra Nova Bay region, northern Victoria Land, Antarctica. Geografia Fisica e Dinamica Quaternaria, 13:139-163.

Orsi, A. H. and Whitworth, T. (2004). Hydrographic Atlas of the World Ocean Circulation Experiment (WOCE), Volume 1: Southern Ocean. International WOCE Project Office, Southampton, UK.

Pagani, M., Zachos, J. C., Freeman, K. H., Tipple, B., and Bohaty, S. (2005). Marked decline in atmospheric carbon dioxide concentrations during the Paleogene. Science, 309(5734):600-603. 
Pahnke, K. and Sachs, J. (2006). Sea surface temperatures of southern midlatitudes 0-160 kyr B.P. Paleoceanography, 21(PA2003).

Paillard, D., Labeyrie, L., and Yiou, P. (1996). Macintosh program performs time-series analysis. Eos, Transactions American Geophysical Union, 77(39):379-379.

Parrenin, F., Masson-Delmotte, V., Köhler, P., Raynaud, D., Paillard, D., Schwander, J., Barbante, C., Landais, A., Wegner, A., and Jouzel, J. (2013). Synchronous change of atmospheric $\mathrm{CO}_{2}$ and Antarctic temperature during the last deglacial warming. Science, 339(6123):1060-1063.

Passchier, S., Browne, G., Field, B., Fielding, C., Krissek, L., Panter, K., Pekar, S., et al. (2011). Early and middle Miocene Antarctic glacial history from the sedimentary facies distribution in the AND-2A drill hole, Ross Sea, Antarctica. Geological Society of America Bulletin, 123(11-12):2352-2365.

Patterson, M., McKay, R., Naish, T., Escutia, C., Jimenez-Espejo, F., Raymo, M., Meyers, S., Tauxe, L., Brinkhuis, H., Expedition, I., et al. (2014). Orbital forcing of the East Antarctic ice sheet during the Pliocene and Early Pleistocene. Nature Geoscience, 7(11):841-847.

Pattyn, F. (2010). Antarctic subglacial conditions inferred from a hybrid ice sheet/ice stream model. Earth and Planetary Science Letters, 295:451-461.

Pattyn, F., Huyghe, A., De Brabander, S., and De Smedt, B. (2006). Role of transition zones in marine ice sheet dynamics. Journal of Geophysical Research: Earth Surface (2003-2012), 111(F2).

Payne, A. J., Vieli, A., Shepherd, A. P., Wingham, D. J., and Rignot, E. (2004). Recent dramatic thinning of largest West Antarctic ice stream triggered by oceans. Geophysical Research Letters, 31(23).

Pekar, S. F. and DeConto, R. M. (2006). High-resolution ice-volume estimates for the early Miocene: Evidence for a dynamic ice sheet in Antarctica. Palaeogeography, Palaeoclimatology, Palaeoecology, 231(1):101-109.

Peltier, L. C. (1950). The geographic cycle in periglacial regions as it is related to climatic geomorphology. Annals of the association of American Geographers, 40(3):214-236.

Peltier, W. R. (2004). Global glacial isostasy and the surface of the ice-age earth: The ICE-5G (VM2) model and GRACE. Annual Review of Earth and Planetary Sciences, 32:111-149.

Peltier, W. R. (2005). On the hemispheric origins of meltwater pulse 1a. Quaternary Science Reviews, 24(14-15):1655-1671.

Petit, J.-R., Jouzel, J., Raynaud, D., Barkov, N. I., Barnola, J.-M., Basile, I., Bender, M., Chappellaz, J., Davis, M., Delaygue, G., et al. (1999). Climate and atmospheric history of the past 420,000 years from the Vostok ice core, Antarctica. Nature, 399(6735):429436.

Pigati, J. S. and Lifton, N. A. (2004). Geomagnetic effects on time-integrated cosmogenic nuclide production with emphasis on in situ ${ }^{14} \mathrm{C}$ and ${ }^{10} \mathrm{Be}$. Earth and Planetary Science Letters, 226(1):193-205. 
Pingree, K., Lurie, M., and Hughes, T. (2011). Is the East Antarctic ice sheet stable? Quaternary Research, 75(3):417-429.

Pollard, D., DeConto, R., and Nyblade, A. (2005). Sensitivity of Cenozoic Antarctic ice sheet variations to geothermal heat flux. Global and Planetary Change, 49(1-2):63-74.

Pollard, D. and DeConto, R. M. (2003). Antarctic ice and sediment flux in the Oligocene simulated by a climate-ice sheet-sediment model. Palaeogeography Palaeoclimatology Palaeoecology, 198(1-2):53-67.

Pollard, D. and DeConto, R. M. (2009). Modelling West Antarctic ice sheet growth and collapse through the past five million years. Nature, 458:329-332.

Powell, R. D., Dawber, M., McInnes, J. N., and Pyne, A. R. (1996). Observations of the grounding-line area at a floating glacier terminus. Annals of Glaciology, 22:217-223.

Prebble, J., Raine, J., Barrett, P., and Hannah, M. (2006). Vegetation and climate from two Oligocene glacioeustatic sedimentary cycles (31 and $24 \mathrm{Ma}$ ) cored by the Cape Roberts Project, Victoria Land Basin, Antarctica. Palaeogeography, Palaeoclimatology, Palaeoecology, 231(1):41-57.

Price, S. F. and Whillans, I. M. (2001). Crevasse patterns at the onset to Ice Stream B, West Antarctica. Journal of Glaciology, 47(156):29-36.

Pritchard, H., Ligtenberg, S., Fricker, H., Vaughan, D., Van den Broeke, M., and Padman, L. (2012). Antarctic ice-sheet loss driven by basal melting of ice shelves. Nature, 484(7395):502-505.

Pritchard, H. D., Arthern, R. J., Vaughan, D. G., and Edwards, L. A. (2009). Extensive dynamic thinning on the margins of the Greenland and Antarctic ice sheets. Nature, 461(7266):971-975.

Putnam, A., Schaefer, J., Barrell, D., Vandergoes, M., Denton, G., Kaplan, M., Finkel, R., Schwartz, R., Goehring, B., and Kelley, S. (2010). In situ cosmogenic ${ }^{10}$ Be productionrate calibration from the Southern Alps, New Zealand. Quaternary Geochronology, 5(4):392-409.

Radok, U., Allison, I., and Wendler, G. (1996). Atmospheric surface pressure over the interior of Antarctica. Antarctic Science, 8(02):209-217.

Ramsey, C. B. (1998). Probability and dating. Radiocarbon, 40(1):461-474.

Ramsey, C. B. (2008). Deposition models for chronological records. Quaternary Science Reviews, 27(1):42-60.

Raymo, M., Lisiecki, L., and Nisancioglu, K. H. (2006). Plio-Pleistocene ice volume, Antarctic climate, and the global $\delta^{18} \mathrm{O}$ record. Science, 313(5786):492-495.

Raymo, M. E. and Mitrovica, J. X. (2012). Collapse of polar ice sheets during the stage 11 interglacial. Nature, 483(7390):453-456.

Raymo, M. E., Mitrovica, J. X., O’Leary, M. J., DeConto, R. M., and Hearty, P. J. (2011). Departures from eustasy in Pliocene sea-level records. Nature Geoscience, 4(5):328332. 
Rebesco, M., Domack, E., Zgur, F., Lavoie, C., Leventer, A., Brachfeld, S., Willmott, V., Halverson, G., Truffer, M., Scambos, T., et al. (2014). Boundary condition of grounding lines prior to collapse, Larsen-B Ice Shelf, Antarctica. Science, 345(6202):1354-1358.

Riger-Kusk, M. (2011). Ice dynamics of the Darwin-Hatherton glacial system, Transantarctic Mountains, Antarctica. Unpublished Ph.D thesis, University of Canterbury, Christchurch.

Rignot, E., Bamber, J. L., van den Broeke, M. R., Davis, C., Li, Y., Van De Berg, W. J., and Van Meijgaard, E. (2008). Recent Antarctic ice mass loss from radar interferometry and regional climate modelling. Nature Geoscience, 1(2):106-110.

Rignot, E., Casassa, G., Gogineni, P., Krabill, W., Rivera, A., and Thomas, R. (2004). Accelerated ice discharge from the Antarctic Peninsula following the collapse of Larsen B ice shelf. Geophysical Research Letters, 31(18).

Rignot, E., Jacobs, S., Mouginot, J., and Scheuchl, B. (2013). Ice-shelf melting around Antarctica. Science, 341(6143):266-270.

Rignot, E., Mouginot, J., and Scheuchl, B. (2011). Ice Flow of the Antarctic Ice Sheet. Science, 333:1427-1430.

Rignot, E. and Thomas, R. H. (2002). Mass balance of polar ice sheets. Science, 297(5586):1502-1506.

Roberts, A. P., Wilson, G. S., Harwood, D. M., and Verosub, K. L. (2003). Glaciation across the Oligocene-Miocene boundary in southern McMurdo Sound, Antarctica: new chronology from the CIROS-1 drill hole. Palaeogeography, Palaeoclimatology, Palaeoecology, 198(1):113-130.

Rohling, E., Grant, K., Bolshaw, M., Roberts, A., Siddall, M., Hemleben, C., and Kucera, M. (2009). Antarctic temperature and global sea level closely coupled over the past five glacial cycles. Nature Geoscience, 2(7):500-504.

Russell, A. and McGregor, G. R. (2010). Southern hemisphere atmospheric circulation: impacts on Antarctic climate and reconstructions from Antarctic ice core data. Climatic change, 99(1-2):155-192.

Scambos, T., Haran, T., Fahnestock, M., Painter, T., and Bohlander, J. (2007). MODISbased Mosaic of Antarctica (MOA) data sets: Continent-wide surface morphology and snow grain size. Remote Sensing of Environment, 111(2):242-257.

Scambos, T. A., Bohlander, J., Shuman, C., and Skvarca, P. (2004). Glacier acceleration and thinning after ice shelf collapse in the Larsen B embayment, Antarctica. Geophysical Research Letters, 31(18).

Schoof, C. (2007). Ice sheet grounding line dynamics: Steady states, stability, and hysteresis. Journal of Geophysical Research: Earth Surface (2003-2012), 112(F3).

Seroussi, H., Morlighem, M., Rignot, E., Mouginot, J., Larour, E., Schodlok, M., and Khazendar, A. (2014). Sensitivity of the dynamics of Pine Island Glacier, West Antarctica, to climate forcing for the next 50 years. The Cryosphere, 8(5):1699-1710.

Shackleton, N. J., Kennett, J. P., et al. (1975). Paleotemperature history of the Cenozoic and the initiation of Antarctic glaciation: oxygen and carbon isotope analyses in DSDP Sites 277, 279, and 281. Initial reports of the deep sea drilling project, 29:743-755. 
Shakun, J. D., Clark, P. U., He, F., Marcott, S. A., Mix, A. C., Liu, Z., Otto-Bliesner, B., Schmittner, A., and Bard, E. (2012). Global warming preceded by increasing carbon dioxide concentrations during the last deglaciation. Nature, 484(7392):49-54.

Shaw, J. (1988). Subglacial erosional marks, Wilton Creek, Ontario. Canadian Journal of Earth Sciences, 25(8):1256-1267.

Shepherd, A., Ivins, E. R., Geruo, A., Barletta, V. R., Bentley, M. J., Bettadpur, S., Briggs, K. H., Bromwich, D. H., Forsberg, R., and Galin, N. (2012). A reconciled estimate of ice-sheet mass balance. Science, 338(6111):1183-1189.

Shevenell, A., Ingalls, A., Domack, E., and Kelly, C. (2011). Holocene Southern Ocean surface temperature variability west of the Antarctic Peninsula. Nature, 470(7333):250-254.

Shevenell, A. E., Kennett, J. P., and Lea, D. W. (2004). Middle Miocene southern ocean cooling and Antarctic cryosphere expansion. Science, 305(5691):1766-1770.

Shipp, S., Anderson, J., and Domack, E. (1999). Seismic signature of the Late Pleistocene fluctuation of the West Antarctic Ice Sheet system in the Ross Sea: a new perspective, Part I. Geological Society of America Bulletin, 111:1486-1516.

Shreve, R. (1984). Glacier sliding at subfreezing temperatures. Journal of Glaciology, 30(106):341-347.

Siegert, M. J., Barrett, P., Deconto, R., Dunbar, R., O'Cofaigh, C., Passchier, S., and Naish, T. (2008). Recent advances in understanding Antarctic climate evolution. Antarctic Science, 20(04):313-325.

Sjunneskog, C. and Winter, D. (2012). A diatom record of late Pliocene cooling from the Ross Sea continental shelf, AND-1B, Antarctica. Global and Planetary Change, 96:87-96.

Smellie, J., Rocchi, S., Gemelli, M., Di Vincenzo, G., and Armienti, P. (2011). A thin predominantly cold-based Late Miocene East Antarctic ice sheet inferred from glaciovolcanic sequences in northern Victoria Land, Antarctica. Palaeogeography, Palaeoclimatology, Palaeoecology, 307(1):129-149.

Smith, J. A., Hillenbrand, C.-D., Kuhn, G., Larter, R. D., Graham, A. G., Ehrmann, W., Moreton, S. G., and Forwick, M. (2011). Deglacial history of the West Antarctic Ice Sheet in the western Amundsen Sea embayment. Quaternary Science Reviews, 30(5):488-505.

Staiger, J. W., Marchant, D. R., Schaefer, J. M., Oberholzer, P., Johnson, J. V., Lewis, A. R., and Swanger, K. M. (2006). Plio-Pleistocene history of Ferrar Glacier, Antarctica: Implications for climate and ice sheet stability. Earth and Planetary Science Letters, 243(3-4):489-503.

Stanford, J., Hemingway, R., Rohling, E., Challenor, P., Medina-Elizalde, M., and Lester, A. (2011). Sea-level probability for the last deglaciation: A statistical analysis of farfield records. Global and Planetary Change, 79(3):193-203.

Stearns, L. A. (2011). Dynamics and mass balance of four large East Antarctic outlet glaciers. Annals of Glaciology, 52:116-126. 
Stearns, L. A., Smith, B. E., and Hamilton, G. S. (2008). Increased flow speed on a large East Antarctic outlet glacier caused by subglacial floods. Nature Geoscience, 1:827831.

Steig, E. J., Brook, E. J., White, J. W. C., Sucher, C. M., Bender, M. L., Lehman, S. J., Morse, D. L., Waddington, E. D., and Clow, G. D. (1998). Sychronous climate changes in Antarctica and the North Atlantic. Science, 282:92-95.

Steig, E. J., Morse, D. L., Waddington, E. D., Stuiver, M., Grootes, P. M., Mayewski, P. A., Twickler, M. S., and Whitlow, S. I. (2000). Wisconsinan and Holocene climate history from an ice core at Taylor Dome, western Ross Embayment, Antarctica. Geografiska Annaler: Series A, Physical Geography, 82(2-3):213-235.

Steig, E. J., Schneider, D. P., Rutherford, S. D., Mann, M. E., Comiso, J. C., and Shindell, D. T. (2009). Warming of the Antarctic ice-sheet surface since the 1957 International Geophysical Year. Nature, 457(7228):459-462.

Stenni, B., Buiron, D., Frezzotti, M., Albani, S., Barbante, C., Bard, E., Barnola, J., Baroni, M., Baumgartner, M., and Bonazza, M. (2011). Expression of the bipolar see-saw in Antarctic climate records during the last deglaciation. Nature Geoscience, 4(1):46-49.

Stern, T., Baxter, A., and Barrett, P. (2005). Isostatic rebound due to glacial erosion within the Transantarctic Mountains. Geology, 33(3):221-224.

Stolldorf, T., Schenke, H.-W., and Anderson, J. B. (2012). LGM ice sheet extent in the Weddell Sea: evidence for diachronous behavior of Antarctic Ice Sheets. Quaternary Science Reviews, 48:20-31.

Stone, J. O. (2000). Air pressure and cosmogenic isotope production. Journal of Geophysical Research: Solid Earth (1978-2012), 105(B10):23753-23759.

Stone, J. O., Balco, G. A., Sugden, D. E., Caffee, M. W., Sass III, L. C., Cowdery, S. G., and Siddoway, C. (2003). Holocene deglaciation of Marie Byrd Land, West Antarctica. Science, 299:99-102.

Storey, B. C., Fink, D., Hood, D., Joy, K., Shulmeister, J., Riger-Kusk, M., and Stevens, M. I. (2010). Cosmogenic nuclide exposure age constraints on the glacial history of the Lake Wellman area, Darwin Mountains, Antarctica. Antarctic Science, 22(6):603-618.

Stuiver, M., Denton, G. H., Hughes, T. J., and Fastook, J. L. (1981). History of the marine ice sheet in West Antarctica during the last glaciation: A working hypothesis. The Last Great Ice Sheets, pages 319-436.

Suganuma, Y., Miura, H., Zondervan, A., and Okuno, J. (2014). East Antarctic deglaciation and the link to global cooling during the Quaternary: evidence from glacial geomorphology and ${ }^{10} \mathrm{Be}$ surface exposure dating of the Sør Rondane Mountains, Dronning Maud Land. Quaternary Science Reviews, 97:102-120.

Sugden, D. and Denton, G. (2004). Cenozoic landscape evolution of the Convoy Range to Mackay Glacier area, Transantarctic Mountains: Onshore to offshore synthesis. Geological Society of America Bulletin, 116(7-8):840-857. 
Sugden, D. E., Balco, G., Cowdery, S. G., Stone, J. O., and Sass, L. C. (2005). Selective glacial erosion and weathering zones in the coastal mountains of Marie Byrd Land, Antarctica. Geomorphology, 67(3):317-334.

Sugden, D. E., Denton, G. H., and Marchant, D. R. (1991). Subglacial meltwater channel systems and ice sheet overriding, Asgard Range, Antarctica. Geografiska Annaler. Series A. Physical Geography, pages 109-121.

Sugden, D. E., Marchant, D. R., and Denton, G. H. (1993). The case for a stable East Antarctic ice sheet: the background. Geografiska Annaler. Series A. Physical Geography, pages 151-154.

Swanger, K. M., Marchant, D. R., Schaefer, J. M., Winckler, G., and Head, J. W. (2011). Elevated East Antarctic outlet glaciers during warmer-than-present climates in southern Victoria Land. Global and Planetary Change, 79(1):61-72.

Swithinbank, C. (1969). Radio echo sounding by the British Antarctic Survey. The Geographical Journal, 139(4):551-553.

Swithinbank, C. W. (1963). Ice movement of valley glaciers flowing into the Ross Ice Shelf, Antarctica. Science, 141(3580):523-524.

Talarico, F. M., McKay, R. M., Powell, R. D., Sandroni, S., and Naish, T. (2012). Late Cenozoic oscillations of Antarctic ice sheets revealed by provenance of basement clasts and grain detrital modes in ANDRILL core AND-1B. Global and Planetary Change, 96:23-40.

Talarico, F. M. and Sandroni, S. (2009). Provenance signatures of the Antarctic Ice Sheets in the Ross Embayment during the Late Miocene to Early Pliocene: the ANDRILL AND-1B core record. Global and Planetary Change, 69(3):103-123.

Tarasov, L., Dyke, A. S., Neal, R. M., and Peltier, W. (2012). A data-calibrated distribution of deglacial chronologies for the North American ice complex from glaciological modeling. Earth and Planetary Science Letters, 315:30-40.

Tarasov, L. and Peltier, W. (2005). Arctic freshwater forcing of the Younger Dryas cold reversal. Nature, 435(7042):662-665.

Tarasov, L. and Peltier, W. (2006). A calibrated deglacial drainage chronology for the North American continent: evidence of an Arctic trigger for the Younger Dryas. Quaternary Science Reviews, 25(7):659-688.

Thomas, E. R., Marshall, G. J., and McConnell, J. R. (2008). A doubling in snow accumulation in the western Antarctic Peninsula since 1850. Geophysical Research Letters, 35(1):5 (L01706).

Thorn, C. E. and Hall, K. (2002). Nivation and cryoplanation: the case for scrutiny and integration. Progress in Physical Geography, 26(4):533-550.

Todd, C., Stone, J., Conway, H., Hall, B., and Bromley, G. (2010). Late Quaternary evolution of Reedy Glacier, Antarctica. Quaternary Science Reviews, 29:1328-1341.

Toggweiler, J. and Russell, J. (2008). Ocean circulation in a warming climate. Nature, 451(7176):286-288. 
Tzedakis, P., Wolff, E., Skinner, L., Brovkin, V., Hodell, D., McManus, J. F., and Raynaud, D. (2012). Can we predict the duration of an interglacial? Climate of the Past, 8(5):1473-1485.

van den Broeke, M. (2005). Strong surface melting preceded collapse of Antarctic Peninsula ice shelf. Geophysical Research Letters, 32(12).

van den Broeke, M., van de Berg, W. J., and Van Meijgaard, E. (2006). Snowfall in coastal West Antarctica much greater than previously assumed. Geophysical Research Letters, 33(2):L02505.

Vaughan, D., Comiso, J., Allison, I., Carrasco, J., Kaser, G., Kwok, R., Mote, P., Murray, T., Paul, F., Ren, J., et al. (2013). Observations: Cryosphere. In Climate Change 2013: The Physical Science Basis. Contribution of Working Group I to the Fifth Assessment Report of the Intergovernmental Panel on Climate Change, pages 317-318. Cambridge University Press, Cambridge, United Kingdom.

Velicogna, I. (2009). Increasing rates of ice mass loss from the Greenland and Antarctic ice sheets revealed by GRACE. Geophysical Research Letters, 36:L19503.

Vieli, A., Funk, M., and Blatter, H. (2001). Flow dynamics of tidewater glaciers: a numerical modelling approach. Journal of Glaciology, 47(159):595-606.

Vieli, A. and Nick, F. M. (2011). Understanding and modelling rapid dynamic changes of tidewater outlet glaciers: issues and implications. Surveys in Geophysics, 32(4-5):437458.

Vliet-Lanoë, V. et al. (1988). The significance of cryoturbation phenomena in environmental reconstruction. Journal of Quaternary Science, 3(1):85-96.

Vliet-Lanoë, V. et al. (1991). Differential frost heave, load casting and convection: converging mechanisms; a discussion of the origin of cryoturbations. Permafrost and Periglacial Processes, 2(2):123-139.

von Blanckenburg, F., Belshaw, N. S., and O’Nions, R. K. (1996). Separation of ${ }^{9}$ Be and cosmogenic ${ }^{10} \mathrm{Be}$ from environmental materials and SIMS isotope dilution analysis. Chemical Geology, 129(1-2):93-99.

von Blanckenburg, F., Hewawasam, T., and Kubik, P. W. (2004). Cosmogenic nuclide evidence for low weathering and denudation in the wet, tropical highlands of Sri Lanka. Journal of Geophysical Research, 109(F03008).

Waddington, E., Conway, H., Steig, E., Alley, R., Brook, E., Taylor, K., and White, J. (2005). Decoding the dipstick: Thickness of Siple Dome, West Antarctica, at the last glacial maximum. Geology, 33(4):281.

WAIS Divide Project Members (2013). Onset of deglacial warming in West Antarctica driven by local orbital forcing. Nature, 500(7463):440-444.

Weber, M., Clark, P., Kuhn, G., Timmermann, A., Sprenk, D., Gladstone, R., Zhang, X., Lohmann, G., Menviel, L., and Chikamoto, M. (2014). Millennial-scale variability in Antarctic ice-sheet discharge during the last deglaciation. Nature.

Weber, M. E., Clark, P. U., Ricken, W., Mitrovica, J. X., Hostetler, S. W., and Kuhn, G. (2011). Interhemispheric ice-sheet synchronicity during the Last Glacial Maximum. Science, 334:1265-1269. 
Weertman, J. (1974). Stability of the junction of an ice sheet and an ice shelf. Journal of Glaciology, 13:3-11.

Wellner, J., Heroy, D., and Anderson, J. (2006). The death mask of the Antarctic ice sheet: comparison of glacial geomorphic features across the continental shelf. Geomorphology, 75(1):157-171.

White, D., Fülöp, R.-H., Bishop, P., Mackintosh, A., and Cook, G. (2011a). Can in-situ cosmogenic ${ }^{14} \mathrm{C}$ be used to assess the influence of clast recycling on exposure dating of ice retreat in Antarctica? Quaternary Geochronology, 6(3):289-294.

White, D. A., Fink, D., and Gore, D. B. (2011b). Cosmogenic nuclide evidence for enhanced sensitivity of an East Antarctic ice stream to change during the last deglaciation. Geology, 39:23-26.

Whitehouse, P. L., Bentley, M. J., and Le Brocq, A. M. (2012). A deglacial model for Antarctica: geological constraints and glaciological modelling as a basis for a new model of Antarctic glacial isostatic adjustment. Quaternary Science Reviews, 32:1-24.

Wilson, C. R. and Crary, A. P. (1961). Ice movement studies on the Skelton Glacier. Journal of Glaciology, pages 873-878.

Wilson, G. S., Levy, R. H., Naish, T. R., Powell, R. D., Florindo, F., Ohneiser, C., Sagnotti, L., Winter, D. M., Cody, R., Henrys, S., et al. (2012). Neogene tectonic and climatic evolution of the Western Ross Sea, Antarctica - Chronology of events from the AND-1B drill hole. Global and Planetary Change, 96:189-203.

Winkelmann, R., Levermann, A., Martin, M. A., and Frieler, K. (2012). Increased future ice discharge from Antarctica owing to higher snowfall. Nature, 492(7428):239-242.

Winter, D., Sjunneskog, C., Scherer, R., Maffioli, P., Riesselman, C., and Harwood, D. (2012). Pliocene-Pleistocene diatom biostratigraphy of nearshore Antarctica from the AND-1B drillcore, McMurdo Sound. Global and Planetary Change, 16:2012.

Winter, D. M., Sjunesskog, C., and Harwood, D. (2010). Early to mid-Pliocene environmentally constrained diatom assemblages from the AND-1B drillcore, McMurdo Sound, Antarctica. Stratigraphy, 7:207-227.

Yokoyama, Y., Lambeck, K., De Deckker, P., Johnston, P., and Fifield, L. K. (2000). Timing of the Last Glacial Maximum from observed sea-level minima. Nature, 406(6797):713-716.

Young, N. E., Schaefer, J. M., Briner, J. P., and Goehring, B. M. (2013). A ${ }^{10} \mathrm{Be}$ production-rate calibration for the Arctic. Journal of Quaternary Science, 28(5):515526.

Zachos, J., Pagani, M., Sloan, L., Thomas, E., and Billups, K. (2001). Trends, rhythms, and aberrations in global climate 65 Ma to present. Science, 292(5517):686-693.

Ziegler, L., Constable, C., Johnson, C., and Tauxe, L. (2011). PADM2M: a penalized maximum likelihood model of the 0-2 Ma palaeomagnetic axial dipole moment. Geophysical Journal International, 184(3):1069-1089.

Zoet, L. K., Anandakrishnan, S., Alley, R. B., Nyblade, A. A., and Wiens, D. A. (2012). Motion of an Antarctic glacier by repeated tidally modulated earthquakes. Nature Geoscience, 5(9):623-626. 
Zweck, C., Zreda, M., and Desilets, D. (2013). Snow shielding factors for cosmogenic nuclide dating inferred from Monte Carlo neutron transport simulations. Earth and Planetary Science Letters, 379:64-71. 


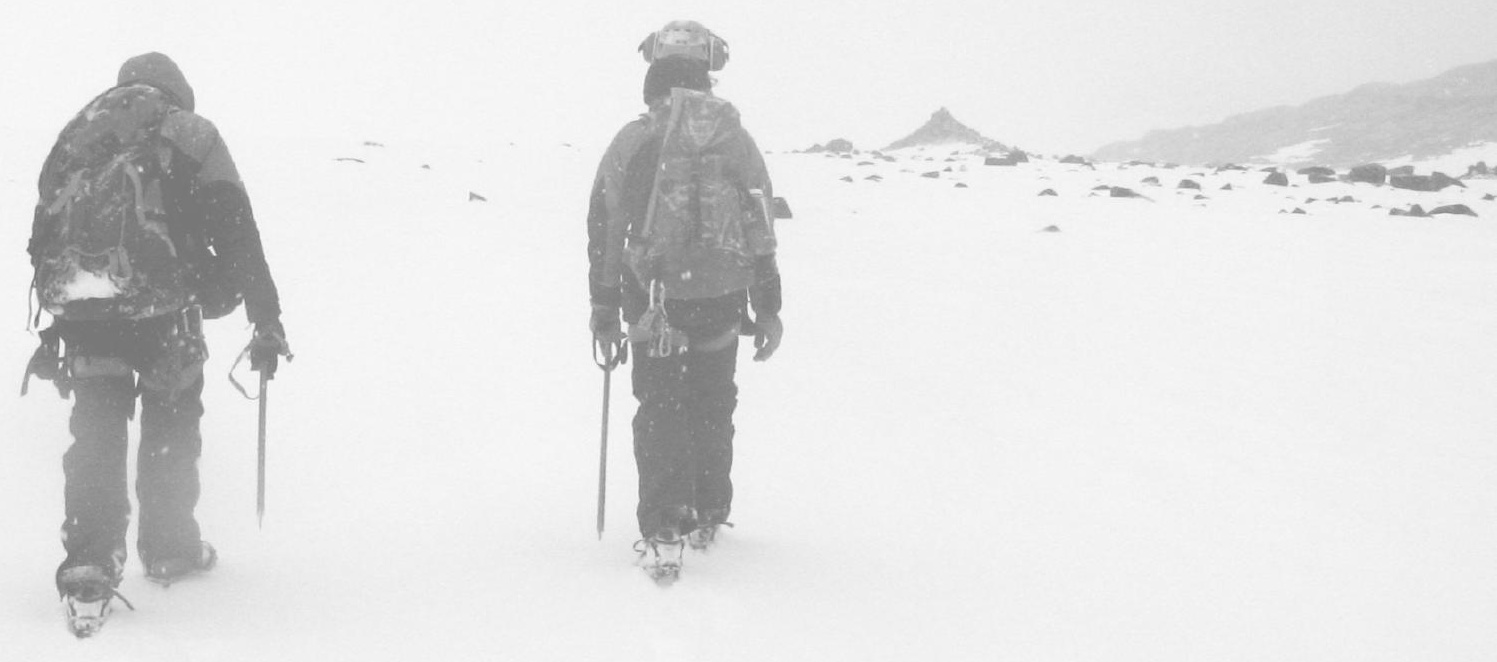

